\title{
CONCEPTUAL DESIGN OF A MODULAR MORPHING WING
}

\author{
by
}

\author{
Allan Daniel Finistauri \\ Bachelor of Engineering, Ryerson University \\ Toronto, Ontario, Canada, 2005
}

\author{
A dissertation \\ presented to Ryerson University \\ in partial fulfillment of the \\ requirements for the degree of \\ Doctor of Philosophy \\ in the Program of \\ Aerospace Engineering
}

Toronto, Ontario, Canada, 2013

(C)Allan Daniel Finistauri, 2013 


\section{Author's Declaration}

I hereby declare that I am the sole author of this dissertation. This is a true copy of the dissertation, including any required final revisions, as accepted by my examiners.

I authorize Ryerson University to lend this dissertation to other institutions or individuals for the purpose of scholarly research.

I further authorize Ryerson University to reproduce this dissertation by photocopying or by other means, in total or in part, at the request of other institutions or individuals for the purpose of scholarly research.

I understand that my dissertation may be made electronically available to the public. 


\section{Borrower's Page}

Ryerson University requires the signatures of all persons using or photocopying this these. Please sign below and give address and date.

\begin{tabular}{|c|c|c|c|}
\hline Name & Signature & Address & Date \\
\hline & & & \\
\hline & & & \\
\hline & & & \\
\hline & & & \\
\hline & & & \\
\hline & & & \\
\hline & & & \\
\hline & & & \\
\hline & & & \\
\hline & & & \\
\hline & & & \\
\hline & & & \\
\hline & & & \\
\hline & & & \\
\hline & & & \\
\hline & & & \\
\hline & & & \\
\hline & & & \\
\hline & & & \\
\hline & & & \\
\hline & & & \\
\hline & & & \\
\hline & & & \\
\hline & & & \\
\hline & & & \\
\hline & & & \\
\hline & & & \\
\hline
\end{tabular}




\title{
Abstract
}

\author{
Conceptual Design of a Modular Morphing Wing \\ Allan Daniel Finistauri \\ A dissertation for the degree of \\ Doctor of Philosophy, 2013 \\ Department of Aerospace Engineering \\ Ryerson University
}

In this dissertation a new modular design method for morphing wings is presented. First, a design method was created, applying modularity and reconfigurability to a morphing wing system. With modularity being a requirement for the morphing wing system, a discretization method is developed to determine the discrete number of modules required to perform a desired morphing maneuver. Then, a specialized, modular, reconfigurable variable geometry truss mechanism is proposed to facilitate morphing. The specialized modular wing truss is a reconfigurable, limited mobility parallel mechanism, adapted to fit within the volume of a wing. The mobility of the wing truss module is analyzed via a branch-based mobility and connectivity analysis that imposes kinematic requirements on the truss mechanism. The mobility and connectivity requirements are used to perform an enumeration analysis to isolate candidate module configurations for morphing. Then, a parametric kinematic constraint system is developed and applied to the wing module and the kinematic performance of the module is evaluated. The kinematics are applied to a mechanical prototype of the wing module for validation purposes. Finally, the kinematics are used to evaluate the motion response of a wing skin system to lay the foundation for detailed design. 


\section{Acknowledgment}

First and foremost, I would like to thank Dr. Jeff Xi who has given me the opportunity to not only take part in, but lead in the development of the morphing wing project. Under his guidance, our research group have been able to accomplish amazing feats, all of which would not have occurred had he not given me the opportunity to present our ideas to Bombardier Aerospace. His dedication to high quality research and his guidance has allowed me not only to publish my works, but present them abroad. These experiences are invaluable not only to my dissertation, but also to develop into a quality research. He is an asset to Ryerson University and the Department of Aerospace Engineering. He is phenomenal Professor on both a professional and personal level and students like myself are lucky to have had the opportunity to learn from him. I am thus indebted for his guidance and encouragement which has allowed me to achieve my best possible work.

Secondly, I would like thank Fassi Kafeyette from Bombardier Aerospace, and the National Science and Engineering Research Council of Canada for their financial support of the Morphing Wing Project. The acquisition of this NSERC-CRD grant has allowed me to attend two conferences abroad, but also has allowed Dr. Xi to put together a top quality team of research students. We have been successful in developing two mechanical prototypes, and various wind tunnel models, which without the financial support provided by Bombardier Aerospace and NSERC, would not have been possible.

Next I would like to thank two specific professors from the Department of Aerospace Engineering. First, I would like to thank Dr. Paul Walsh for guidance with the aerodynamics portion of my work. I would also like to thank Dr. Krishna Kumar for his financial support during the early portion of my academic career. 
Thanks must also be given to my fellow graduate students in EPH 107. Being locked up in a windowless room for eight or more hours a day will without doubt drive some to the brink of insanity. Periodic breaks for Dim Sum or for a pint or two (or three) helped us all keep on an even keel. The friendship and camaraderie with the other graduate students allowed me to enjoy my time as a graduate student. Particularly thanks must be given to Richard Mohamed. We have been colleagues and friends since our time as undergraduate engineering students, and it was on his advice that I talk to Dr. Jeff Xi about pursuing my Masters, then Doctoral degree. My thanks goes out to Richard as my successes could not have been accomplished without his advice.

Saving the best for last, I would like to thank my beautiful wife Whitney, who has always been loving and encouraging. Her love has given me the confidence and support I have needed to complete my work. She has worked harder than anyone in supporting me through my degree and I look forward to returning the favour as we raise our first child. This work is dedicated to you and Amelia. 


\section{Contents}

Author's Declaration $\quad$ ii

Borrower's Page $\quad$ iii

$\begin{array}{ll}\text { Abstract } & \text { iv }\end{array}$

Acknowledgment $\quad$ v

Table of Contents vii

List of Tables $\quad$ xii

List of Figures $\quad$ xiv

$\begin{array}{ll}\text { Nomenclature } & \text { xviii }\end{array}$

1 Introduction $\quad 1$

1.1 Morphing Aircraft Technology . . . . . . . . . . . . . . . . . . 1

1.2 Aircraft Wing Morphing . . . . . . . . . . . . . . . . . 4

1.2 .1 Benefits of Wing Morphing . . . . . . . . . . . . . . . 4

1.2.2 Airfoil-Level vs. Wing-Level Morphing . . . . . . . . . . . . . . . . 5

1.2.3 Characteristics of Wing-Level Morphing . . . . . . . . . . . . . . 6

1.2.4 Challenges Associated with Wing Morphing . . . . . . . . . . . . . . . 7

1.3 Problem Statement . . . . . . . . . . . . . . . . . . . . . . . 8

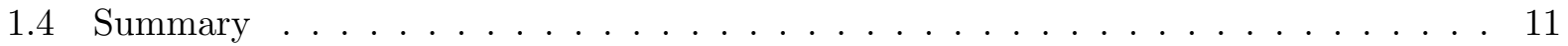


2.1 Morphing Wing Technology . . . . . . . . . . . . . . . . . . . 12

2.1.1 Airfoil-Level Morphing . . . . . . . . . . . . . . . . . . . 12

2.1 .2 Wing-Level Morphing . . . . . . . . . . . . . . . 13

2.2 Actuation Methods for Wing-Level Morphing . . . . . . . . . . . . . . . . 22

2.2.1 Single Degree-of-Freedom Mechanisms . . . . . . . . . . . . . . . . 22

2.2.2 Poly-Morphing Wing Mechanisms . . . . . . . . . . . . . . . 24

2.3 Summary of Morphing Wing Technology Surveyed . . . . . . . . . . . . . . 24

2.4 Mechanism Overview . . . . . . . . . . . . . . . . . . . 25

2.4 .1 Mechanism Style for Wing Morphing . . . . . . . . . . . . . . . 26

2.4 .2 Truss Mechanism Overview . . . . . . . . . . . . . . 27

2.4.3 Reconfigurable Parallel-Style Mechanisms . . . . . . . . . . . . . . . . 30

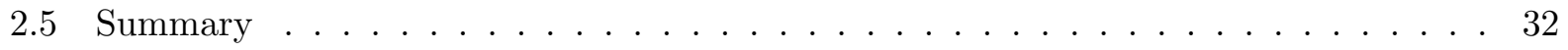

3 Wing Module Discretization $\quad 34$

3.1 Morphing Wing System Overview . . . . . . . . . . . . . . . . . 34

3.1.1 Joint Placement in Morphing Wing Mechanisms . . . . . . . . . . . . 35

3.1.2 Requirements of Modular Morphing Wings . . . . . . . . . . . . . 36

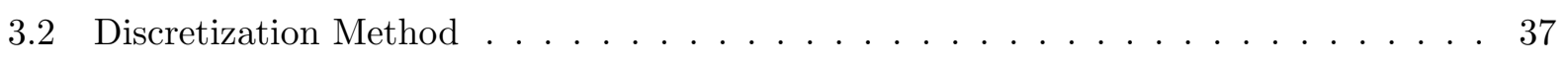

3.2 .1 Discretization Algorithm . . . . . . . . . . . . . . 37

3.2.2 Geometry Discretization . . . . . . . . . . . . . . . 40

3.2 .3 Performance Analysis . . . . . . . . . . . . . . . . . . 4 41

3.3 Differential Curve Geometry . . . . . . . . . . . . . . . . . . . . . 42

viii 
3.3 .1 Curve Framing . . . . . . . . . . . . . . . . . . . . . 42

3.3 .2 Frenet-Serret Frame . . . . . . . . . . . . . . . . . . . . 44

3.3.3 Parallel-Transport Frame . . . . . . . . . . . . . . . . 45

3.3.4 Curve Frame Comparison . . . . . . . . . . . . . . . 47

3.4 Wing Curve Discretization . . . . . . . . . . . . . . . . . . . 48

3.4 .1 Wing Curve Framing . . . . . . . . . . . . . . . . . . . 48

3.4 .2 Differential Wing Curve Geometry . . . . . . . . . . . . . . . 50

3.4 .3 Module Discretization . . . . . . . . . . . . . . . 53

3.4 .4 Kinematic Implementation . . . . . . . . . . . . . . . . . . 54

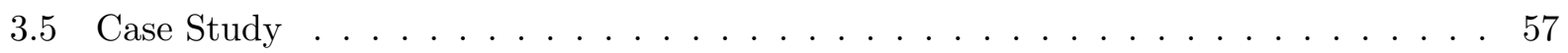

3.5.1 Reference Wing Curve Definition . . . . . . . . . . . . . . 57

3.5.2 Morphing Wing Geometry Discretization . . . . . . . . . . . . . . 58

3.5.3 Morphing Wing Performance Evaluation . . . . . . . . . . . . . . . . 61

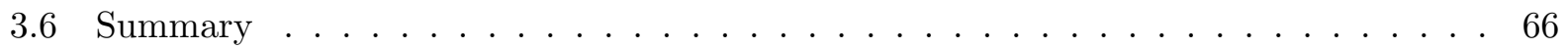

4 Wing Module Kinematic Architecture $\quad 68$

4.1 Parallel Mechanism Branch Mobility, Connectivity and Design Methodologies . . . . 68

4.1.1 Parallel Mechanism Mobility and Connectivity . . . . . . . . . . . . 69

4.1.2 Parallel Mechanisms Constraint Methodology . . . . . . . . . . . . . 70

4.2 Wing Module Design Methodology . . . . . . . . . . . . . . 73

4.2.1 Branch Constraint Wing Module . . . . . . . . . . . . . . . 73

4.2 .2 Joint Constraint Wing Module . . . . . . . . . . . . . . . . . 82

4.2 .3 Module Comparison . . . . . . . . . . . . . . . . 88 
4.2.4 Passive Wing Module . . . . . . . . . . . . . . . . . 89

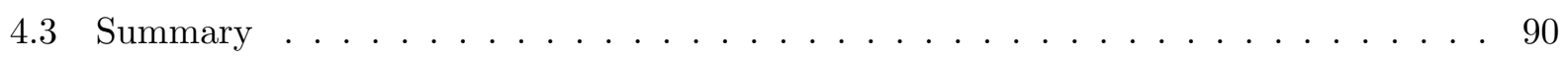

$5 \quad$ Wing Module Kinematic Modeling $\quad 91$

5.1 Wing Module Kinematic Setup . . . . . . . . . . . . . . . . . . 91

5.2 Module Kinematic Formulation . . . . . . . . . . . . . . . . . . 94

5.2 .1 Parametric Constraint Formulation . . . . . . . . . . . . . . . . 94

5.2 .2 Constraint Solving . . . . . . . . . . . . . . . . 96

5.2 .3 Instantaneous Kinematics . . . . . . . . . . . . . . . . . 103

5.2 .4 Parametric Velocity Constraint . . . . . . . . . . . . . 106

5.3 Kinematic Evaluation $\ldots \ldots \ldots \ldots \ldots$

5.3 .1 Module Kinematic Range . . . . . . . . . . . . . . . . . . . . . . 108

5.3.2 Module Constrained Jacobian Inverse Condition Distribution . . . . . . . . . 127

5.3 .3 Kinematics Summary . . . . . . . . . . . . . . . 143

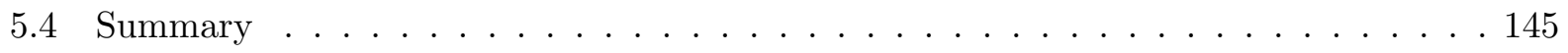

6 Wing Module Prototype Development 146

6.1 Module Prototype . . . . . . . . . . . . . . . . . 146

6.1 .1 Module System Description . . . . . . . . . . . . . . 146

6.1 .2 Kinematic Response . . . . . . . . . . . . . . . . . . 148

6.2 Wing Module Skin System Conceptual Design . . . . . . . . . . . . . . . 151

6.2 .1 Morphing Wing Skin Systems . . . . . . . . . . . . . . . . . 152

6.2.2 Wing Skin Kinematic Response . . . . . . . . . . . . . . . . 154

6.2 .3 Wing Skin Motion Summary . . . . . . . . . . . . . 162

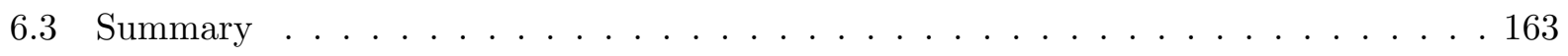


7.1 Conclusions . . . . . . . . . . . . . . . . . . . . 164

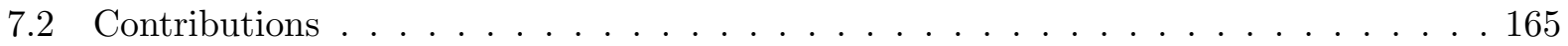

7.3 Future Work . . . . . . . . . . . . . . . . . . 168

$\begin{array}{ll}\text { Appendices } & 171\end{array}$

$\begin{array}{lr}\text { A CFD Mesh } & 172\end{array}$

B Parallel Robot Branch Enumeration $\quad 175$

B.1 Parallel Mechanism Branch Candidate Enumeration . . . . . . . . . . . . . 175

B.2 Parallel Mechanism Constrained Branch Candidate Enumeration . . . . . . . . . . 179

$\begin{array}{lr}\text { C Prototype Motion Video } & 183\end{array}$

$\begin{array}{ll}\text { References } & 184\end{array}$ 


\section{List of Tables}

1.1 Characteristics of wing-level morphing . . . . . . . . . . . . . . 7

2.1 Relative comparison of mechanism archetypes . . . . . . . . . . . . . 27

3.1 Points used to form wing curve b-spline . . . . . . . . . . . . . . 57

3.2 Nodal point distribution and module kinematics of the a selection of discretized wings 59

4.1 Mobility and connectivity summary of typical parallel robots $\ldots \ldots \ldots$. . . . . 72

4.2 Mobility and connectivity comparison of typical constraint branch parallel robots . . 74

4.3 Constraint branch joint sequence summary $\ldots \ldots \ldots \ldots \ldots$

4.4 Enumeration of controllable branch combinations for the constraint branch wing module. . . . . . . . . . . . . . . . . . . . . 79

4.5 Enumeration of final configurations for the constraint branch wing module . . . . . 82

4.6 Mobility and connectivity comparison of typical joint constraint parallel robots . . . 83

4.7 Enumeration of all possible wing module configurations . . . . . . . . . . 85

4.8 Enumeration of final wing module configurations $\ldots \ldots \ldots \ldots$

5.1 Wing module branch parameters . . . . . . . . . . . . . . 108

5.2 Kinematic range for the 4 -DOF wing module configurations at $b_{z}=250 \mathrm{~mm} \ldots . . .109$

5.3 Kinematic range for the 3 -DOF wing module configurations $\ldots \ldots \ldots$ 
5.4 Kinematic range for the 2 -DOF wing module configurations . . . . . . . . . . 125

5.5 Constrained Jacobian inverse condition data for the 4-DOF wing module configura-

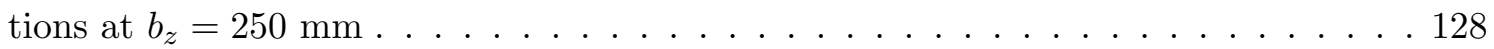

5.6 Constrained Jacobian inverse condition data for the 3-DOF wing module configurations with dependent platform twist $\ldots \ldots \ldots \ldots \ldots \ldots$

5.7 Constrained Jacobian inverse condition data for the 2-DOF wing module configurations138

6.1 Firgelli Technologies, Inc L16 150:1 $140 \mathrm{~mm}$ actuator characteristics . . . . . . . . 149

B.1 Summary of common kinematic pairs . . . . . . . . . . . . . 176

B.2 Enumeration of all possible parallel robot branch candidates with two-link, threejoint branch architecture . . . . . . . . . . . . . . 177

B.3 Enumeration of feasible parallel robot branch candidates with two-link, three-joint

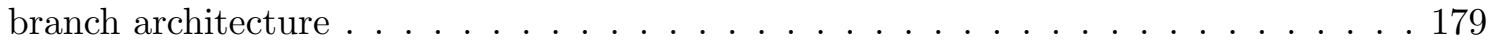

B.4 Reconfiguration of branch candidates for reduced mobility modes . . . . . . . . 181 


\section{List of Figures}

1.1 Sketches of mechanical wings proposed by Leonardo da Vinci for the purpose of

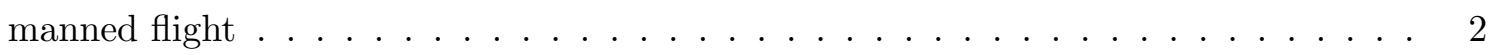

1.2 Diagrams of successful morphing aircraft . . . . . . . . . . . . . . . . 3

1.3 Wing-level morphing maneuvers $\ldots \ldots \ldots \ldots \ldots$

1.4 Venn diagram of morphing wing degrees-of-freedom . . . . . . . . . . . . . . 10

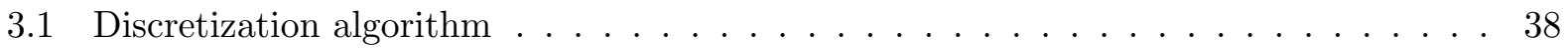

3.2 Discretization algorithm example of a wing curve defining a bird gliding close to the

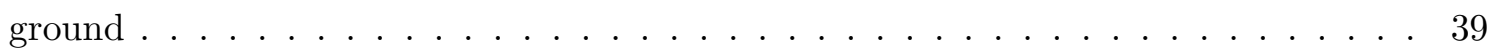

3.3 Aircraft forces in steady level flight . . . . . . . . . . . . . . . . . 41

3.4 Space curve showing infinite number of curve frames $\ldots \ldots \ldots$

3.5 Frenet-Serret moving frame and osculating plane . . . . . . . . . . . . . 45

3.6 Parallel vector field . . . . . . . . . . . . . . . . . . . . 46

3.7 Comparison between Frenet-Serret and parallel transport frames moving along a

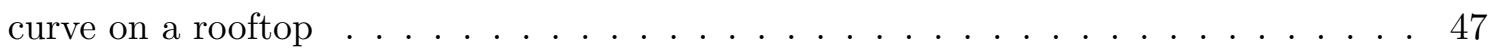

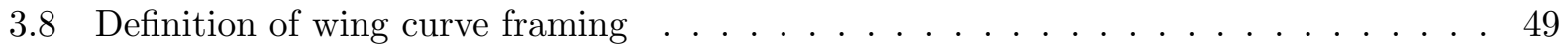

3.9 Wing module showing successive sweep, cant and twist rotations . . . . . . . . 51

3.10 Definition of module twist $\ldots \ldots \ldots \ldots \ldots$ 
3.11 Definition of reference wing used for discretization algorithm case study $\ldots \ldots$. . . 58

3.12 Total curvature distribution of the reference wing curve showing nodal point distribution for selected wing configurations . . . . . . . . . . . . 61

3.13 Wing module average variable values . . . . . . . . . . . . . . . . . 61

3.14 Wing configurations for case study discretization algorithm example . . . . . . . 63

3.15 Case study performance evaluation results showing lift, drag and lift-to-drag ratio

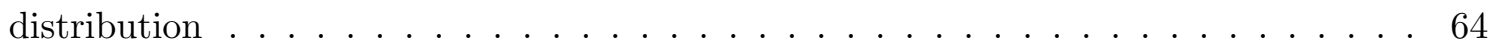

3.16 Example morphing maneuver showing a transition from a straight, untwisted wing, to a fully morphed state . . . . . . . . . . . . . . 65

3.17 Pressure coefficient distribution for three discretized wing configurations $\ldots$. . . . 66

4.1 Example of translation then rotation branch constraint module with $15^{\circ}$ sweep, $15^{\circ}$ cant, $-5^{\mathrm{o}}$ twist, and 12 unit span $\ldots \ldots \ldots \ldots \ldots$

4.2 Example of rotation then translation branch constraint module with $15^{\circ}$ sweep, $15^{\circ}$ cant, $-5^{\mathrm{o}}$ twist, and 12 unit span $\ldots \ldots \ldots \ldots \ldots \ldots$

4.3 Branch constraint wing module with branch numbers and branch airfoil connection

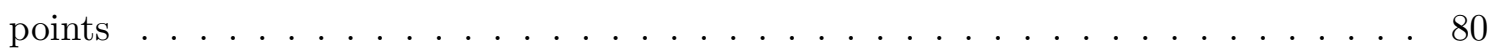

4.4 Constraint branch modules with $1-\mathrm{DOF} \ldots \ldots \ldots \ldots$. . . . . . . . 81

4.5 Joint constraint wing module . . . . . . . . . . . . . . . . . . 84

4.6 Example wing module configurations . . . . . . . . . . . . . . . . 87

5.1 Joint constraint wing module shown branch vector loop . . . . . . . . . . . 92

5.2 Joint constraint wing module shown branch vector loop and constraint plane . . . . 94

5.34 -DOF wing modules with red constrained branches and $\Lambda=-15^{\circ}, \Gamma=30^{\circ}, \alpha=-5^{\circ}$

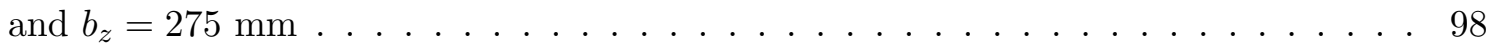


5.43 -DOF twist-constrained wing modules with red constrained branches and $\Lambda=-15^{\circ}$,

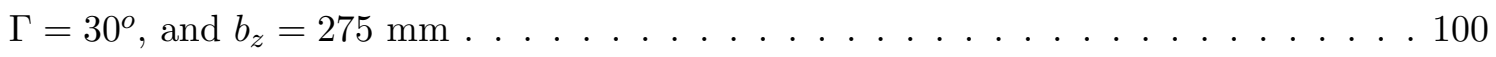

5.52 -DOF wing modules with red constrained branches and $b_{z}=275 \mathrm{~mm} \ldots \ldots 4$

5.6 Sweep range of 4 -DOF wing modules at $b_{z}=250 \mathrm{~mm} \ldots \ldots \ldots$

5.7 Cant range of 4 -DOF wing modules at $b_{z}=250 \mathrm{~mm} \ldots \ldots \ldots \ldots$

5.8 Twist range of 4 -DOF wing modules at $b_{z}=250 \mathrm{~mm} \ldots \ldots \ldots \ldots$

5.9 Sweep range of 3 -DOF wing modules with constrained platform cant $\ldots \ldots$. . . 118

5.10 Sweep range of 3-DOF wing modules with constrained platform twist . . . . . . . 119

5.11 Cant range of 3-DOF wing modules with constrained platform sweep . . . . . . . 120

5.12 Cant range of 3 -DOF wing modules with constrained platform twist . . . . . . 121

5.13 Twist range of 3 -DOF wing modules with constrained platform sweep $\ldots$. . . . . 122

5.14 Twist range of 3 -DOF wing modules with constrained platform cant $\ldots . . . . .124$

5.15 Angular range of the 2 -DOF wing module . . . . . . . . . . . 126

5.16 Constrained Jacobian inverse condition distribution for 4-DOF wing modules at

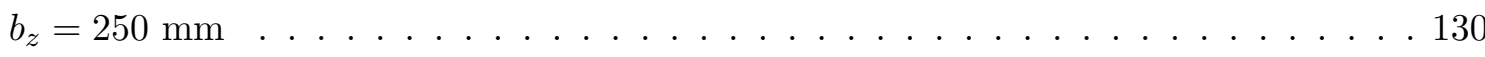

5.17 Constrained Jacobian inverse condition distribution for 2-DOF wing modules with constrained platform twist and $5-6-7$ constrained branches $\ldots \ldots \ldots$. . . . 133

5.18 Constrained Jacobian inverse condition distribution for 2-DOF wing modules with constrained platform twist and $5-6-8$ constrained branches $\ldots \ldots \ldots$. . . . . 134

5.19 Constrained Jacobian inverse condition distribution for 2-DOF wing modules with constrained platform twist and $5-7-8$ constrained branches $\ldots \ldots \ldots$. . . . 135

5.20 Constrained Jacobian inverse condition distribution for 2-DOF wing modules with constrained platform twist and 6-7-8 constrained branches $\ldots \ldots \ldots 136$ 
5.21 Constrained Jacobian inverse condition distribution for 2-DOF wing modules with active platform sweep . . . . . . . . . . . . . . . . . . 140

5.22 Constrained Jacobian inverse condition distribution for 2-DOF wing modules with active platform cant . . . . . . . . . . . . . . . . . . 141

5.23 Constrained Jacobian inverse condition distribution for 2-DOF wing modules with active platform twist . . . . . . . . . . . . . . . . . . . 142

6.1 Mechanical prototype of wing module . . . . . . . . . . . . . . 147

6.2 Screen shot of wing module prototype control interface . . . . . . . . . . . . 150

6.3 Wing module prototype with single morphing degree-of-freedom pose . . . . . . . 150

6.4 Wing module prototype with multiple degree-of-freedom pose, utilizing 4 degrees-of-

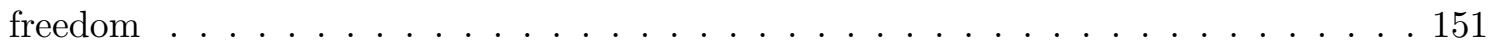

6.5 Wing module showing skin grid . . . . . . . . . . . . 156

6.6 Wing skin motion response for 4-DOF wing module with 5-6 constrained branches with module sweep at $b_{z}=250 \mathrm{~mm} \ldots \ldots \ldots \ldots$. . . . . . . . . . . . . . . . . .

6.7 Wing skin motion response for 4-DOF wing module with 5-6 constrained branches with module cant at $b_{z}=250 \mathrm{~mm} \ldots \ldots \ldots \ldots$. . . . . . . . . . . . . . . . .

6.8 Wing skin motion response for 4-DOF wing module with 5-6 constrained branches with module twist at $b_{z}=250 \mathrm{~mm} \ldots \ldots \ldots \ldots$

6.9 Wing skin motion response for 4-DOF wing module with 5-6 constrained branches

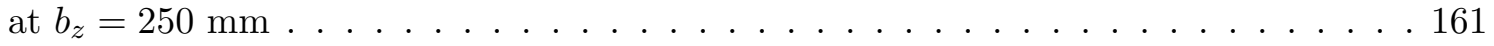

A.1 Computational grid used for the discretization algorithm performance analysis _ . . 173 


\title{
Nomenclature
}

\author{
$\alpha \quad$ Module twist \\ $\bar{\zeta} \quad$ Average wing total curvature \\ $\boldsymbol{\omega} \quad$ Wing module platform airfoil angular velocity \\ $\boldsymbol{\omega}_{\mathbf{d}} \quad$ Wing module branch angular velocity \\ $\chi \quad$ Total curvature \\ $\delta \quad$ Intermediate airfoil section fraction \\ $\Gamma \quad$ Module cant \\ $\kappa \quad$ Curve curvature \\ $\Lambda \quad$ Module sweep \\ $\lambda \quad$ System order \\ $\dot{\mathbf{b}} \quad$ Wing module platform airfoil linear velocity \\ $\dot{\mathbf{d}} \quad$ Wing module branch linear velocity \\ $\dot{\mathbf{d}} \quad$ Wing module joint velocity \\ a Span-wise skin vector \\ B Frenet-Serret frame binormal \\ b Module span
}

xviii 


\begin{tabular}{|c|c|}
\hline d & Wing module branch vector \\
\hline $\mathbf{J}$ & Jacobian \\
\hline $\mathbf{J}_{\mathrm{t}}$ & Twist Jacobian \\
\hline $\mathbf{J}_{c}$ & Constrained Jacobian \\
\hline $\mathbf{J}_{d e p}$ & Dependent velocity Jacobian \\
\hline $\mathbf{J}_{f w d}$ & Forward Jacobian \\
\hline $\mathbf{J}_{\text {ind }}$ & Independent velocity Jacobian \\
\hline $\mathbf{J}_{i n v}$ & Inverse Jacobian \\
\hline $\mathbf{N}$ & Frenet-Serret frame normal \\
\hline $\mathbf{n}$ & Constrained branch plane normal \\
\hline $\mathbf{N}_{1}, \mathbf{N}_{2}$ & Parallel transport frame normal basis vectors \\
\hline $\mathbf{p}_{a, b}$ & Base airfoil perimeter vector \\
\hline $\mathbf{p}_{a, p}$ & Platform airfoil perimeter vector \\
\hline $\mathbf{p}_{b}$ & Base airfoil branch vector \\
\hline $\mathbf{p}_{p}$ & Platform airfoil branch vector \\
\hline $\mathbf{q}_{D}$ & Dependent platform variable motions \\
\hline $\mathbf{q}_{I}$ & Independent platform variable motions \\
\hline s & Space curve \\
\hline $\mathbf{s}^{\prime}$ & Curve velocity \\
\hline $\mathbf{s}^{\prime \prime}$ & Curve acceleration \\
\hline
\end{tabular}




\begin{tabular}{|c|c|}
\hline $\mathbf{s}_{X}$ & Wing curve $1 / 4$-chord line \\
\hline $\mathbf{T}$ & Frenet-Serret, parallel transport frame tangent \\
\hline $\mathbf{t}$ & Nodal spacing vector \\
\hline $\mathbf{t}_{p}$ & Platform airfoil twist vector \\
\hline $\mathbf{t}_{d e p}$ & Dependent twist vector \\
\hline $\mathbf{t}_{\text {ind }}$ & Independent twist vector \\
\hline $\mathcal{F}$ & Space curve moving coordinate frame \\
\hline $\mathcal{O}_{b}, \mathcal{O}_{p}$ & Wing module base airfoil and platform airfoil coordinate frames, respectively \\
\hline$\mu$ & Condition number \\
\hline$\tau$ & Curve torsion \\
\hline$\zeta$ & Wing total curvature \\
\hline$C$ & Branch connectivity \\
\hline$C_{D}$ & Drag coefficient \\
\hline$C_{L}$ & Lift coefficient \\
\hline$f$ & Joint total degrees-of-freedom \\
\hline$K$ & Constraints \\
\hline$k_{1}, k_{2}$ & Parallel transport frame parameters \\
\hline$M$ & Mobility \\
\hline$m$ & Number of modules \\
\hline$M_{\infty}$ & Free stream Mach number \\
\hline
\end{tabular}




\begin{tabular}{|c|c|}
\hline$n_{b}$ & Number of branches \\
\hline$n_{j}$ & Number of joints \\
\hline$n_{l}$ & Number of links \\
\hline$R e$ & Reynolds number \\
\hline$s_{\theta}$ & Wing curve frame spin \\
\hline$s_{x}, s_{y}, s_{z}$ & $x, y$, and $z$ space curve components, respectively \\
\hline$t$ & Curve parameterization variable \\
\hline$\AA$ & Arc length \\
\hline AoA & Angle of attack \\
\hline $\mathrm{C}$ & Cylindrical joint \\
\hline DOF & Degrees-of-Freedom \\
\hline MAV & Micro air vehicle \\
\hline $\mathrm{P}_{\mathrm{T}}$ & Fixed leg length prismatic joint \\
\hline $\mathrm{P}_{\mathrm{V}}$ & Variable leg length prismatic joint \\
\hline $\mathrm{R}_{\mathrm{A}}$ & Axial axis revolute joint \\
\hline $\mathrm{R}_{\mathrm{T}}$ & Transverse axis revolute joint \\
\hline S & Spherical joint \\
\hline SMA & Smart memory alloy \\
\hline $\mathrm{U}$ & Universal (Hooke) joint \\
\hline UAV & Unmanned air vehicle \\
\hline VGTM & Variable Geometry Truss Mechanism \\
\hline
\end{tabular}




\section{Chapter 1}

\section{Introduction}

In this chapter, an introduction to morphing aircraft technology is discussed with a particular focus on wing morphing technology. First, an introduction to various types of aircraft morphing technology is discussed. Next, aircraft morphing wing technology is discussed in further detail. The benefits of wing morphing are outlined, followed by a comparison between airfoil-level and winglevel morphing. This is followed by a more detailed description of wing-level morphing, including the characteristics and the challenges associated with wing-level morphing. With wing morphing technology introduced, the problem statement of this dissertation is given. Finally, conclusions are given.

\subsection{Morphing Aircraft Technology}

The forefathers of manned flight once used avian morphology as inspiration for the design of their heavier-than-air vehicles. The notion of this was plausible at the time given that the taxonomic class aves, representing birds, had evolved very specific biological features, wings, which provided the means for flight for most species. Originally, the avenue for manned flight was through manpowered flapping wing flying machines, or ornithoptors. Leonardo da Vinci at the end of the $15^{\text {th }}$ century proposed several mechanisms shown in his sketches (depicted in Fig. 1.1) of man-powered flying machines that bear a direct resemblance to the skeletal structure of bats and birds.

Many other pioneers of aviation looked to bird and bat morphology for inspiration for their 


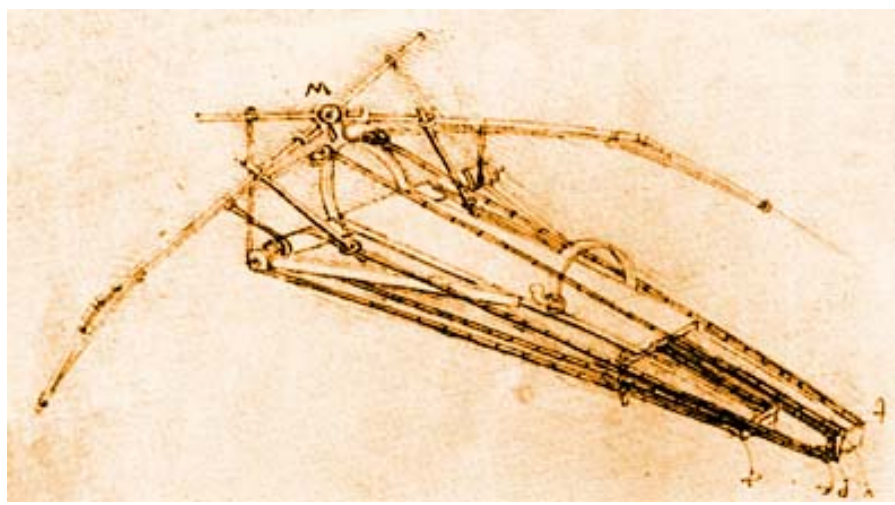

(a) da Vinci's human powered ornithoptor

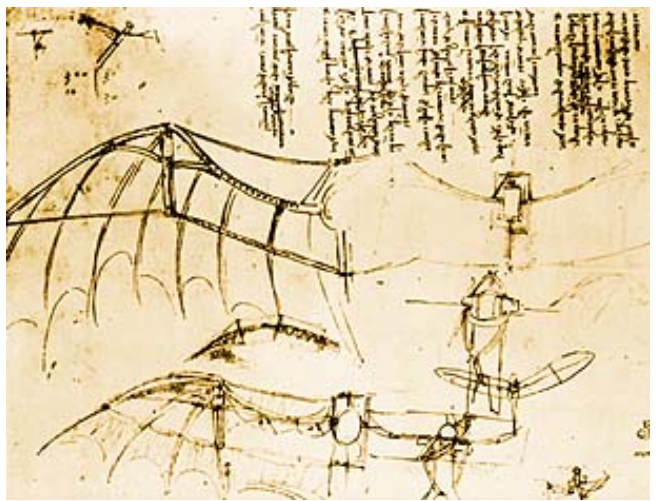

(b) da Vinci's mechanical wing device

Figure 1.1: Sketches of mechanical wings proposed by Leonardo da Vinci for the purpose of manned flight [1]

designs. Some pioneers also investigated gliding flight. However with the successful flight of the Wright Flyer I, powered, fixed-wing aircraft dominated the design scope for the majority of the $20^{\text {th }}$ century. During this time, various high-lift and control surface technologies were developed to enhance fixed-wing aircraft performance and maneuverability. Included are devices such as flaps and slats, ailerons, elevators, etc. There is a valid argument that these devices are in fact, morphing technologies. However their commonplace within the scope of aircraft design, and their necessity for current flight standards, limits the argument that these technologies can currently be categorized as morphing technology. However, these high-lift and control devices can be considered as morphing aircraft technology due to their fundamental purpose of altering the performance of the aircraft by very specific means.

Besides the high-lift and control devices mentioned, other morphing aircraft technology has not had the same success in gaining acceptance within the aircraft design spectrum. Despite this, there are in fact morphing aircraft that have been, or are currently in service, such as the Bell Boeing V22 Osprey, the Aérospatiale-BAC (now BAE-Systems) Concorde, and the Grumman F14 Tomcat. Each of these aircraft utilizes morphing for specific purposes. The Bell Boeing V-22 Osprey's vertical and short takeoff and landing (VSTOL) capabilities are possible due to the variable pitch of the two propellers (Fig. 1.2(a)). The droop-nose feature of the Aérospatiale-BAC Concorde allowed for enhanced pilot visibility during taxiing and landing maneuvers (Fig. 1.2(b)). The Grumman F-14 


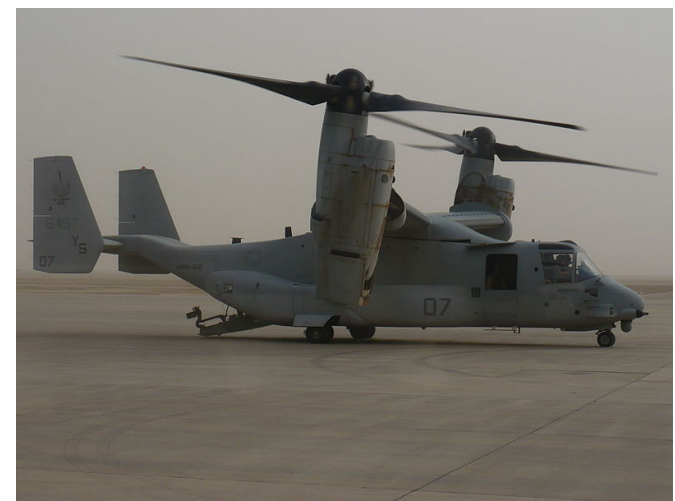

(a) Bell Boeing V-22 Osprey [2]

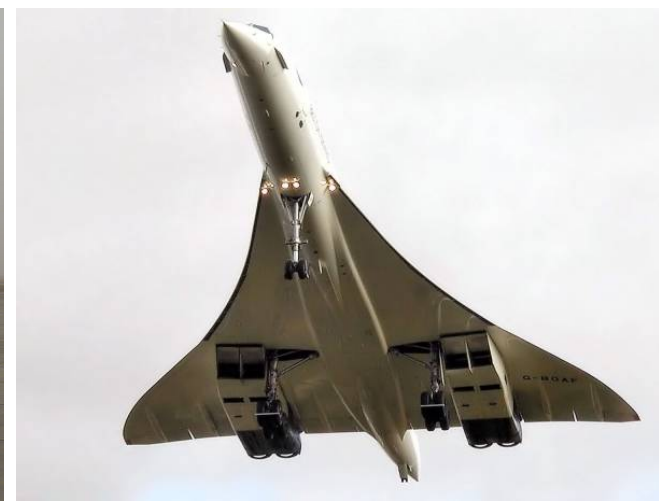

(b) Aérospatiale-BAC Concorde [3]

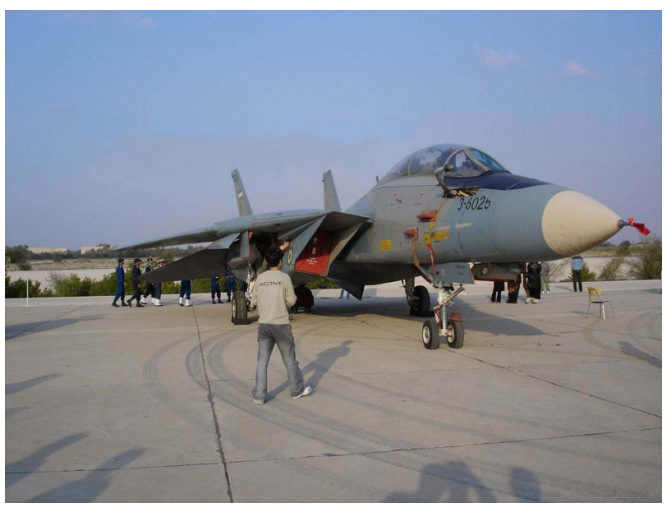

(c) Grumman F-14 Tomcat [4]

Figure 1.2: Diagrams of successful morphing aircraft

Tomcat falls under the category of morphing wing systems, as the variable sweep system allowed for efficient cruise capabilities with fully swept wings, and enhanced maneuverability and interception capabilities with a fully expanded wing (Fig. 1.2(c)).

Other morphing wing aircraft are currently in operation, each with the main goal of enhancing the performance of the aircraft via morphing. The majority of an aircraft's performance is derived from the size and shape of its wing, thus it is the wing that has garnered the majority of interest in terms of developing morphing systems. Here, and throughout the remained of this dissertation, performance is used in a broad sense, encompassing all aspects of flight performance. For example flight performance could be steady or non-steady maneuvers, such as cruise or level turns, respectively, transition from weight-on-wheels to weight-off-wheels and vice versa, such as takeoff and landing, and other on ground requirements such as taxiing and gate parking. Jha and Kudva [5] 
provide an excellent summary of morphing aircraft introduced up until 2004, and provide a classification scheme into which morphing aircraft can be categorized. Included in their report are current technology trends, and challenges associated with morphing aircraft. Rodriguez [6] also provided a technological survey of morphing aircraft in 2007, including an introduction of new technologies that can be applied to the morphing aircraft field.

\subsection{Aircraft Wing Morphing}

Morphing wing technology is categorized into two main groups: a) airfoil-level morphing in which the two-dimensional cross section geometry of an airfoil is altered, and b) wing-level morphing which alters the three-dimensional geometric configuration of the wing. Airfoil-level and winglevel morphing are mutually exclusive and could be used in combination to achieve even greater aircraft performance enhancements as compared to morphing wing systems which uses one method exclusively. A trade-off exists between morphing capabilities and realistic implementation of these system. As such, for simplicity and focus, most developed morphing systems concentrate exclusively on either airfoil-level or wing-level morphing. This is in lieu of creating systems so complex that any performance gains achieved through morphing are mitigated due to overly complex systems. There exists, however, great opportunities and their associative challenges for enhanced aircraft performance via wing morphing, which is discussed in the following sections. In this dissertation, a morphing maneuver refers to the specific morphing motion of the wing system that transforms to wing itself from one geometric state to another.

\subsubsection{Benefits of Wing Morphing}

There is a clear benefit to morphing as an aircraft with morphing capabilities can achieve performance greater than what can be achieved by its baseline, non-morphing counterpart. In effect, morphing has the potential to transform a single purpose or single mission aircraft into one that is multi-purpose, capable of multiple missions, each with drastically different profiles. An example of this potential was studied by Joshi et al. [7] in which the performance of a baseline Firebee 
aircraft was compared to ones with airfoil-level and wing-morphing capabilities. In their study, various flight profiles are used as a basis for comparing the performance of the three Firebee aircraft; the baseline aircraft, the fixed-wing aircraft retrofitted with airfoil morphing capabilities, and the aircraft with wing morphing capabilities. In their analysis, it is shown that both airfoillevel and wing-level morphing outperform the baseline aircraft for almost all flight profiles, clearly highlighting the benefits associated with morphing.

\subsubsection{Airfoil-Level vs. Wing-Level Morphing}

Before delving into wing-level morphing, it is worth comparing the fundamental differences between airfoil-level and wing-level morphing. The obvious distinction between the two are the physical parameters of the wing which are altered for each morphing paradigm; airfoil-level morphing alters the two-dimensional cross section characteristics of wing, whereas wing-level morphing alters the three-dimensional characteristics of the wing. From an analysis perspective, both airfoil-level and wing-level morphing affect the three-dimensional flow over the wing. For wing-level morphing this is obvious as the large-scale displacements of the wing will drastically alter the flow. For airfoil-level, the two-dimensional morphing of airfoil geometry is extended over a finite span of the wing, thus affected the three-dimensional flow characteristics of the wing. For instance, the performance of a flap can be analyzed aerodynamically in two-dimensions. However the physical flap is obviously a three-dimensional object with a finite span and would requires a three-dimensional flow analysis on a wing for its full aerodynamic effects. It is still characterized as an airfoil-level morphing device because the morphing mechanism alters the two-dimensional geometry of the airfoil, and the morphed body simply extends out span-wise along the wing. Wing-level morphing, on the other hand, generally, cannot be analyzed with two-dimensional airfoil theory as the effects of morphing are realized on a three-dimensional scale. For instance, a wing with twist-morphing capabilities alters the span-wise angle-of-attack (AoA) distribution. Locally, the two-dimensional shape of the airfoil has not been altered, thus twist-morphing is considered to be a wing-level morphing maneuver. 
The difference between airfoil-level and wing-level morphing lies in the ability for each morphing paradigm to improve the performance of the wing. The study of Joshi et al. [7] shows that for nearly all flight profiles, wing-level morphing outperforms airfoil-level morphing. This result is perhaps expected based on common aircraft shapes; an endurance-efficient aircraft such as a glider has much different shape than an aircraft expected to perform high-g maneuvers, the former having long straight wings with positive dihedral, and the latter having shorter wings, possibly with negative dihedral. As such, the ability to drastically alter the shape of the wing offers the aircraft greater potential to not only increase its performance for various flight regimes, but also offers the aircraft the opportunity to be multi-purpose. An aircraft with airfoil-level morphing capabilities is less likely to be able to fly beyond its initial role, despite its ability to attain improved performance. Winglevel morphing was therefore chosen as the desired avenue to developing a wing morphing system as will be highlighted in the Section 1.3. Before the problem statement of this dissertation is defined, detailed characteristics of wing morphing and the challenges of wing morphing are addressed.

\subsubsection{Characteristics of Wing-Level Morphing}

As mentioned, wing-level morphing is a large scale morphing maneuver that dramatically alters the three-dimensional configuration of the wing without altering the airfoil configuration. The configuration of a wing from a three-dimensional perspective is described geometrically in terms of its sweep, cant (or dihedral), twist and span. For morphing, each geometric feature listed above is considered to be a separate and distinct degree-of-freedom (DOF) possessed by the wing. These morphing degrees-of-freedom are shown, along with a baseline aircraft for reference, in Fig. 1.3. Here we see that sweep (Fig. 1.3(b)), cant (Fig. 1.3(c)), and twist (Fig. 1.3(d)) are all mutually orthogonal rotational motions. Span (Fig. 1.3(e)) on the other hand is a linear translational motion. Each morphing degree-of-freedom alters the wing in different ways, thus it is expected that each will affect the aircraft performance in different ways. These impacts are briefly summarized in Table 1.1. 
Table 1.1: Characteristics of wing-level morphing [8-10]

\begin{tabular}{|c|c|c|c|}
\hline $\begin{array}{l}\text { Morphing } \\
\text { Type }\end{array}$ & $\begin{array}{l}\text { Performance } \\
\text { Effects }\end{array}$ & Benefit & Cost \\
\hline Sweep & $\begin{array}{l}\text { Drag-divergence } \\
\text { Mach number }\end{array}$ & $\begin{array}{l}\text { Useful for performing dash } \\
\text { maneuvers, high-speed flight }\end{array}$ & $\begin{array}{l}\text { Lower lift coefficient, higher } \\
\text { weight }\end{array}$ \\
\hline Cant & Lateral stability & $\begin{array}{l}\text { Positive cant increases lateral } \\
\text { (roll) stability while negative } \\
\text { cant increases } \\
\text { maneuverability, possibility } \\
\text { for instantaneous winglets to } \\
\text { reduce induced drag }\end{array}$ & $\begin{array}{l}\text { Increased stability results in } \\
\text { decreased maneuverability } \\
\text { and vice versa }\end{array}$ \\
\hline Twist & $\begin{array}{l}\text { Lift, drag and } \\
\text { moment }\end{array}$ & $\begin{array}{l}\text { Control of aerodynamic forces } \\
\text { and moments, ability to } \\
\text { maintain level body, useful as } \\
\text { high lift and control surface }\end{array}$ & $\begin{array}{l}\text { Lower wing torsional rigidity } \\
\text { and moment }\end{array}$ \\
\hline Span & $\begin{array}{l}\text { Aspect ratio and } \\
\text { wing loading }\end{array}$ & $\begin{array}{l}\text { Larger aspect ratio increases } \\
\text { performance parameters, } \\
\text { shorter span increase } \\
\text { maneuverability }\end{array}$ & $\begin{array}{l}\text { Large span results in large } \\
\text { wing root moments }\end{array}$ \\
\hline
\end{tabular}

\subsubsection{Challenges Associated with Wing Morphing}

Aircraft wing morphing cannot be successfully implemented without addressing several challenges that arise when adding a mechanical morphing mechanism to a structural wing. Costs associated with specific wing morphing degrees-of-freedom are also briefly summarized in Table 1.1.

One particular challenge is the development and implementation of suitable morphing skin systems. From a wing-level morphing perspective, each of the four main morphing capabilities impose different requirements on the skin system. Sweep, cant, twist and span-morphing result in

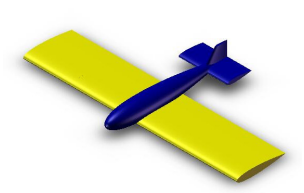

(a) Baseline

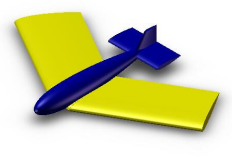

(b) Sweep

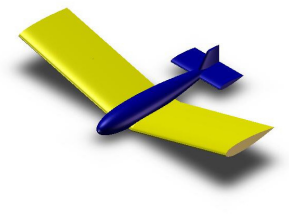

(c) Cant

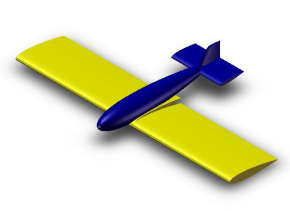

(d) Twist

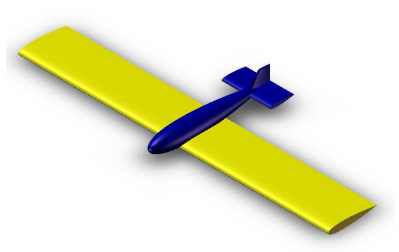

(e) Span

Figure 1.3: Wing-level morphing maneuvers 
motions that are predominantly shearing, bending, torsional and extension/retraction, respectively, thus the skin system must be able to adequately support these motions. Any combination of morphing degrees-of-freedom places additional and more complex requirements on the skin system. Regardless of the morphing motion, a wing morphing skin system must $a$ ) maintain the aerodynamic integrity of the wing, $b$ ) distribute pressure and shear force distribution to the supporting structure, and c) avoid imposing additional requirements or constraints on the morphing mechanism (i.e. increase in motor torque requirement).

\subsection{Problem Statement}

Aircraft morphing at the airfoil or wing level aims to improve the performance of the aircraft, and augment the capabilities of the aircraft from single-purpose to multi-purpose. A versatile multi-purpose morphing wing is expected to have varying morphing requirements between different regions along the wing. This variation can be addressed with two different design methodologies:

\section{Dedicated Mechanical System}

A system designed specifically to meet the current requirements imposed. For a morphing wing, the dedicated design methodology will result in the development of a morphing wing whose functioning mechanism is tailored to meet the specific morphing requirements imposed. The development process includes the cost of not only determining the appropriate architecture of the mechanism, but also analyzing the motion, strength and dynamics of the mechanism itself. For instance, a morphing wing capable only of varying a single degree-offreedom is dedicated to 1-DOF morphing only. The associative analysis of the morphing wing mechanism structure is valid only for that particular design. The final mechanism design in this case is restrictive in the sense that this system is only capable of meeting the particular requirements that the single degree-of-freedom morphing imposed. A change in the flight requirements such as different or the addition of degree-of-freedoms are required, or that the current morphing mechanism cannot meet the new requirements, results in a wing that is limited by the design, leading to a limitation on performance improvement capabilities. A 
restriction in performance is the reason that one would design a morphing wing. Therefore, we see that in certain cases a dedicated morphing wing mechanism can be a limiting factor in increasing the performance of an aircraft. To successfully address the these issues a new system must be developed and analyzed.

\section{Modular Mechanical System}

A system designed to meet a variety of requirements including not only those that are current, but rather, encompassing all known requirements. For a morphing wing, a modular reconfigurable methodology can be adopted to develop a base design platform which is used as a template for the detailed design of motion-specific modules. The base module is designed to meet all known morphing requirements. As a design platform, the morphing mechanism can quickly be reconfigured to suit the motion requirements on demand. The mechanism architecture, motion, structure and dynamic analyses are developed parametrically with respect to the base module, and adapted accordingly for each reconfigured module permutation. When applied to a full morphing wing system, modules are selected and incorporated into the wing system where they are needed. Any change in flight requirements can be addressed quickly by reconfiguring the modules currently in use, or if necessary, adding more modules to the wing system. Here there are no performance limitations associated with the mechanism design; the wing is configured to meet the current requirements without the need for a specific design. This adaptability within the mechanism design highlights the benefits of developing the system in a modular and reconfigurable manner.

Two common aspects of most morphing wing projects currently in development can be identified. First, the majority of projects focus solely on one type of wing morphing. Second, the majority of morphing concepts are first presented, then developed, and then analyzed for their potential performance improvements. These two aspects, in combination, result in a development environment that is not fully sustainable. First, focusing on a single morphing degree-of-freedom limits the potential performance gains to only those associated with the particular type of morphing (see Table 1.1). The single-purpose, dedicated mechanical wing system that facilitates morphing is 


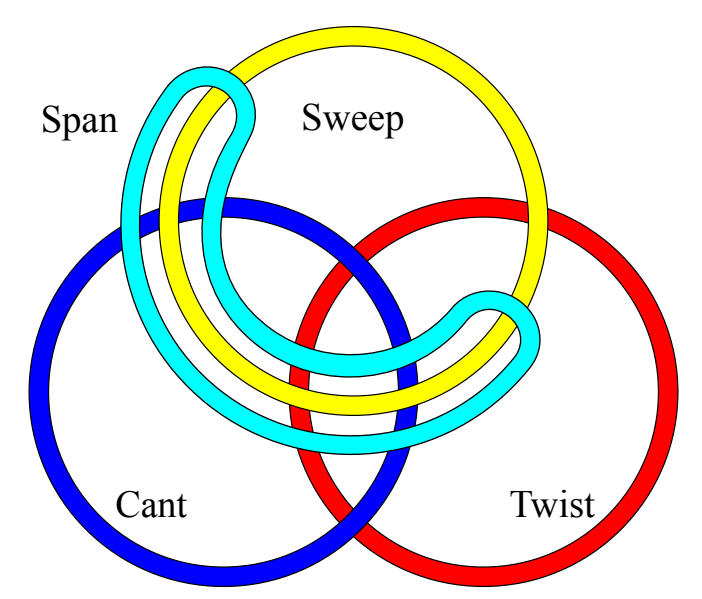

Figure 1.4: Venn diagram of morphing wing degrees-of-freedom

so specific that there is no possibility to study different morphing methodologies using the current form of the wing. A new wing must thus be designed for each case, which in part, negates the purpose of developing morphing wing systems; to enhance the aircraft performance and capabilities beyond its baseline state. Secondly, proposing a design first, followed by its analysis, results in performance improvements of a morphing wing aircraft bound by the functional limits of the morphing wing system itself. Any chance for further performance improvements or expansion of the aircraft's role is not possible due to the original design of the wing.

This dissertation focuses on the conceptual design of a reconfigurable, modular mechanical system for aircraft wing morphing. The objectives of this dissertation are to address the issues previously identified, and are summarized as follows:

\section{Wing Module Discretization}

Develop a method that discretized a continuous wing system, in which an algorithm determines the minimum number of modules required, and their respective span-wise spacing, that achieves near-optimal performance for a specific morphing maneuver.

\section{Wing Module Kinematic Architecture}

Identify the high-level architectural requirements for a modular, reconfigurable mechanism that facilitates multi-morphing capabilities, and address the relevant mobility, connectivity and constraint requirements. 


\section{Wing Module Kinematics}

Create a parametric kinematic model that is applicable to various wing module configurations, and assess the kinematic capabilities of the developed wing mechanism.

\section{Wing Module Prototype Development}

Show through the development of a prototype wing module that the developed kinematic model is valid, and the kinematic model can be used for high-level motion requirements of morphing skin systems.

\subsection{Summary}

In this chapter, morphing aircraft technology was introduced. First, a brief history of morphing aircraft was presented, starting from pioneers of flight, and leading to current examples of morphing aircraft. Next, aircraft wing morphing was introduced. Included, were the benefits of wing-morphing as it pertains to flight performance. Next, a brief comparison between airfoil-level and wing-level morphing was given in which the fundamental difference between these two morphing methodologies was analyzed. Through examples in the literature, wing-level morphing systems were shown to provide better performance improvements over airfoil-level morphing systems. After, wing-level morphing was presented in detail, including the introduction of the four main morphing degrees-of-freedom. Sweep, cant, twist and span morphing were each summarized in terms of their potential performance gains in addition to the challenges associated with each morphing type. This was followed by a brief summary of the requirements for morphing skin systems. With morphing wing technology introduced, the problem statement of this dissertation was presented. 


\section{Chapter 2}

\section{Literature Review}

In this chapter, a detailed literature review is presented on the current trends in morphing wing technology. First, a brief overview of airfoil-level morphing is given. This is followed by a detailed review of the different wing-level morphing types, including variable sweep, cant, twist and span, as well poly-morphing wings and other morphing wing systems. From the literature reviewed, a summary is given on the methods by which morphing is facilitated. Following the review of morphing wing technology, a review of mechanisms is given, focusing on truss mechanisms, their kinematic structure and their application within the morphing wing field. This is followed by a literature review of recent developments within the field of reconfigurable parallel robots. Finally, conclusions are given.

\subsection{Morphing Wing Technology}

\subsubsection{Airfoil-Level Morphing}

A morphing maneuver (transition from one geometric configuration to another) provided by an airfoil-level morphing mechanism, in general, will aim to alter the chord length, airfoil thickness, airfoil camber or possibly the airfoil reflex. As mentioned in Chapter 1, airfoil-level morphing is a technology that is used in every aircraft with high-lift devices and control surfaces as these devices alter the airfoil shape of the wing. For example, simultaneously deploying a flap and slat will significantly alter the airfoil camber and chord length, whereas ailerons essentially alter the camber 
and reflex of the airfoil. Performance improvements are achieved by use of high-lift and control surfaces during specific segments of the prescribed mission. Any additional alterations to the airfoil shape can further enhance the aircraft performance for each mission segment. Many examples of morphing airfoils can be found in the literature but are not reviewed here. The focus of the review is on wing-level morphing and is given next.

\subsubsection{Wing-Level Morphing}

Morphing at the wing-level, in general, aims to alter the sweep, cant, twist and span of some portion of the wing. Any combination of these morphing degrees-of-freedom, referred to as polymorphing, will further provide the aircraft with improved flight performance capabilities and allow it to continuously fly in a more optimal state. The mechanism required to drive a poly-morphing wing will be much more complex as compared to one that is required for a single morphing degreeof-freedom. However, the potential performance gains justify the additional complexity. Many concepts have been proposed that address wing-level morphing, each aiming to address a specific morphing requirement. For each of the individual wing-level morphing degrees-of-freedom introduced in Section 1.2.3 a review of morphing wing aircraft projects is given in the sections that follow. This is followed by a review of poly-morphing wings, and other wing morphing concepts that are not able to be categorized with respect to the individual wing-level morphing degrees-of-freedom.

\subsubsection{Sweep-Morphing Wings}

In general, sweep morphing can be accomplished by rotating regions of the wing about an axis parallel to the aircraft yaw axis, or by translating airfoil rib sections in the direction of the leading or trailing edge of the wing (similar to a shearing motion). The difference between the motions lies in the orientation of airfoil sections with the free stream flow after morphing. For a wing that is swept by rotating the wing, airfoil sections will be orientated at an angle to the free stream flow, whereas a shearing motion translates sections of the wing forward or aft and airfoil sections maintain their initial orientation to the free stream flow. Regardless of the motion, sweep morphing aircraft are capable of flying more efficiently at higher speeds. 
As stated in Section 1.1, one of the most famous sweep-morphing aircraft is the Grumman F14 Tomcat. The wings on the F14 Tomcat rotate aft to morph the aircraft from a conventional wingand-tail configuration into a delta wing configuration. A similar concept was developed by Neal et al. [11] who presented a unique sweep-morphing aircraft named the Morpheus. By means of a four-bar linkage, the sweep angle of the wings can be altered from a straight wing, to an aft-swept wing. A series of aluminum inserts within the outboard section of the wing allows control over the wing twist within the outboard region. As the outboard wing section material is compliant, a smooth wing twist is achievable and acts as a replacement for conventional control surfaces. The addition of the wing twist to the sweep morphing capabilities allow for advanced maneuvering. The most unique feature of the morphing system, and that which makes it different than the F14 Tomcat, is that the triangular tail wing can translate forward to connect with the fully swept wing, reconfiguring the aircraft from a conventional wing-and-tail configuration into a delta wing configuration.

Another example of a sweep-morphing wing was presented by Bharti et al. [12] A cellular truss mechanism was developed that sweeps the wing via a shearing motion. The wing mechanism consist of a series of airfoil ribs that are interconnected by diagonally oriented spars, thus completing the modular truss configuration. Actuation is achieved by means of a spring and cable assembly where the cable is connected to one of the airfoil ribs. The spring forces the wing to sweep aft, and the wing is returned to a straight configuration as the cable is retracted. As the author notes, wear of the cable is possible and could prevent the mechanism from returning to its straight wing state.

Under a DARPA sponsored program, NextGen Aeronautics have developed the MFX-1 morphing aircraft. Using a specialized cellular truss mechanisms, the morphing wing is capable of variable sweep, while simultaneous altering the chord length and taper ratio of the wing. The truss mechanism, which is similar to a scissor-type mechanism, consists of two independent actuation methods for each wing degree-of-freedom. For sweep-morphing, prismatic actuators are situated within the wing and actuation effects the sweep angle by means of a hinge located at the wing root leading edge. The taper ratio of the wing is altered by means of a sliding mechanism that moves 
the wing root trailing edge connection point on the fuselage. By increasing or decreasing the wing root chord length, the cellular truss expands or contracts accordingly, and changes the wing taper ratio while maintaining the wing sweep angle. The trailing edge profile of the wing maintains an 'M' shape to prevent interference between trailing edge sections as the chord length changes [13]. A prototype jet-powered unmanned air vehicle (UAV) was also developed and flight tested [14].

The aircraft listed above all have mechanisms that alter the sweep angle of the full wing. At the University of Florida, a morphing aircraft capable of sweep variation at two locations along the wing has been developed. Instead of having only a single sweeping motion for the entire wing, an additional sweep degree-of-freedom is located near the mid wing position. The aircraft uses two independent motors that drives inboard and outboard wing sections, thus allowing for local sweep control for the two main wing sections. The inboard and outboard sections rotate about axes parallel with the aircraft yaw axis, thus relative motion between the wing sections is experienced. Each wing is actuated independently, allowing for non-symmetric aircraft configurations. The performance of the aircraft was analyzed for various flight regimes, determining optimal wing sweep angles, and highlighting the benefits of morphing. In addition, a small prototype UAV was developed. To provide an aerodynamic surface, strips of carbon fibre overlap in a feather-like fashion and react according to the wing motion [15].

\subsubsection{Cant-Morphing Wings}

Cant, or dihedral-morphing is a versatile morphing maneuver that allows an aircraft to alter it stability or maneuverability and hence, performance. Unlike sweep-morphing in which a shear motion is available, cant morphing is achieved by rotating the wing about an axis parallel to the free stream flow. The variable gull-wing aircraft developed at the University of Florida is an example of this as the morphing aircraft is capable of changing the cant angle of the wing at two discrete locations. Similar to the variable sweep aircraft from the University of Florida listed above, the variable gull-wing is fitted with independently controlled joints at the wing root and mid wing locations. However, unlike the sweep-morphing prototype, the variable gull-wing always performs 
symmetric actuation of the port and starboard wings. Performance analyses and optimal wing configurations were shown for various flight regimes [16] and flight tests of the prototype were also performed, highlighting the performance and response of the aircraft $[17,18]$.

Wings with cant-morphing capabilities also have the ability to provide the aircraft with on demand winglets. For instance, Bourdin et al. [19] presented a flying wing with variable cant angle winglets. The flying wing is fitted with winglets that follow the leading edge and trailing edge sweep profiles of the wing such that in a non morphed state, the aircraft is a continuous flying wing. In their work, a wind tunnel model was tested for the aerodynamic response of winglets that represents $50 \%$ of the wing semi-span, showing the profound impact that morphing has on various aerodynamic properties. The dynamic response of the winglet was also investigated using a modified commercial remote control flying wing [20].

While wing morphing has generally taken inspiration from the morphology of birds, as well as from bats and insects, other bio-inspired morphing wings have also been proposed. A manta ray was used as inspiration for the development of a morphing wing using tensegrity trusses. Moored et al. [21] presented a cellular cable driven truss in which structural integrity and actuation of the truss is achieved via cables in tension, hence tensegrity. In their work, they introduce the truss system to mimic the motion of a mantra ray wing, using three locations in which the local cant angle can be altered. However, the developed prototype consists of solid wing sections with traditional motors. Ueno et al. [22] also developed a cant-morphing wing for a manta ray.

\subsubsection{Twist-Morphing Wings}

Twist-morphing occurs when a portion or the entire wing is rotated about the span-wise axis. Controlling the twist of the wing can be used as a replacement for control surfaces such as ailerons. In general, twist-morphing mechanisms used as control surface replacement are situated on flexible wings. For instance, a twist-morphing wing was developed using smart memory alloys (SMA) to control the twist of the wing. A developed prototype showed how the actuation of an SMA actuator can twist the local region of a flexible wing, altering the twist of the remaining outboard wing [23]. 
A small micro air vehicle (MAV) was recently developed at the University of Florida with twistmorphing capabilities. The small aircraft has flexible wings with torque rods that deflect outboard regions of the wing for roll control [24]. Later, Abdulrahim et al. [25] evaluated flight performance characteristics of this aircraft, analyzing roll maneuvers as well as stability performance. Flight test of a prototype were also performed [26]. Both tests highlight the benefits of morphing for a small remote control air vehicle.

Another aircraft with twist-morphing capabilities at the outboard region of the wing was presented by Guiler and Huebsch [27]. The tailless aircraft uses a torque rod for twist-morphing at the distal regions of the wing for aircraft pitch, roll and yaw control. Instead of using a purely flexible material, a series of floating airfoil ribs are bound together using a flexible material to maintain the structural integrity of the wing, as well as maintain a continuous aerodynamic surface. In their work, they compare the twist-morphing wing to a conventional tailless wing with control surfaces near the tip of the wing via wind tunnel testing. They show that the morphing wing can replace elevons.

While torque rods can be used for twist control of flexible wings, they can also be used for

the twist control of solid wing sections. A prototype aircraft was recently designed to mimic the approach a bird would make in flight prior to perching. The aircraft wing consists of two rigid wing sections, one inboard and one outboard, connected to torque rods through the $1 / 4$-chord line. To successfully emulate the perching maneuver, the inboard and outboard wing sections pitch upward at very high angles-of-attack in order to simulate the orientation of a bird wing as it pitches noseup. A small prototype model was built using identical airfoil shapes for the inboard and outboard wing sections, as well as the body where the actuators were placed [28].

\subsubsection{Span-Morphing Wings}

The final individual morphing degree-of-freedom is span-morphing. Varying the span of the wing affects the wing aspect ratio, which has a direct impact on the wing loading, maneuverability, as 
well as the lift and drag. To accomplish a span-morphing maneuver, the wing must be, in some way, extensible and retractable. Span-morphing is primarily achieved via telescoping mechanisms in which outboard wing sections remain stowed within a hollow inboard wing, and are extended span-wise, thus increasing the overall span of the wing. This allows solid wing skins to cover wing sections, without the need for any specialized flexible skin.

A span-morphing wing as developed using a two-stage, pneumatic, telescoping rod. The outboard wing section is initially stowed within the middle wing section which itself is initially stowed within the inboard wing section. A pneumatic actuator was developed to control the span of the wing and some static testing was preformed to show the structural capabilities of the wing [29]. Later, wind tunnel testing was performed on the wing. Tests were performed using an aluminum foil tape to blend the discontinuities between the wing sections and results were compared to those of the baseline wing; no appreciable differences between the two models were discovered, possibly due to discontinuities being parallel with the free stream flow. Additional wind tunnel analysis was performed analyzing the use of friction tape on the wing surface in attempts to simulate a sliding surface [30].

Telescoping span-morphing wings have also been proposed for use on ballistic missiles. At Virginia Polytechnic and State University, research on the use of telescoping wings have been performed for the main wing of a ballistic missile. In their work Bae et al. [31,32] examine the aerodynamic and structural effects of span morphing, showing that altering the span can increase the range of the long range Cruise missile via drag reduction. Asymmetric wing morphing was also shown to be useful in the roll control of the missile in lieu of using conventional fin ailerons. As missiles are single flight aircraft, there is no ability to reuse the morphing wing for later flights, however the increase range may be an acceptable trade off for the additional development cost.

A small span-morphing UAV was recently developed at the Universidade da Beira Interior. A rack and pinion mechanism drives the outboard wing section from within the hollow inboard wing section to achieve span morphing. Structural testing of a ground model was performed using sand bags to simulate a continuous load distribution on the full span configuration [33]. Asymmetric 
morphing was also investigated as a means for roll control. By morphing each wing individually, the lift distribution ellipse can be shifted from the aircraft centre line, resulting in aircraft roll moments. The span-morphing wing can therefore be used for aircraft maneuvers [34].

Other methods for span-morphing have also been proposed. For instance, a mechanism concept was presented that uses a scissor linkage and sliding beam to extend a wing. Each airfoil rib slides along the beam as the wing extends, and each airfoil is capable of chord and camber-morphing for drag reduction over all flight regimes [35]. A different approach to span-morphing was also attempted by a research group at Konkuk University. Heryawan et al. [36] developed a small wind tunnel model of a biomimetic inspired expandable wing. The aircraft designed resembles a small bird, and the expandable morphing wing consists of an inboard and outboard wing section, connected by a small mechanism that expands the wing. The variation of the wing aspect ratio was shown to have a profound impact on the aerodynamics, however, the developed morphing wing contained discontinuities between wing sections. The gaps between the wing sections could impose penalties on the aircraft with respect to the aerodynamics of the wing, however this was not mentioned or explored. Additionally, in the contracted state, the wing shape is highly irregular as the outboard wing section is rotated such that the $1 / 4$-chord line is parallel to the free stream flow.

\subsubsection{Poly-Morphing Wings}

The morphing wing aircraft listed in the previous sections each focus primarily on a single wing-level morphing degree-of-freedom. Some concepts and prototypes have multiple morphing capabilities, however the additional morphing degree-of-freedom for most prototypes is an implementation of twist-morphing of a flexible wing for control purposes $[11,15-18]$. There are however several morphing aircraft that have been designed specifically with multiple morphing capabilities. These poly-morphing wings have greater potential to enhance the performance capabilities of the aircraft they are placed on as compared to single morphing degree-of-freedom mechanisms.

For instance, a small UAV was designed at the University of Florida using avian morphology 
as a design inspiration. The wing concept combined sweep and cant morphing joints situated at the wing root, and mid-wing positions. The morphing wing concept represented a combination of previous research of a variable sweep wing [15] and a variable cant wing [16-18]. The combined morphing wing setup was used to emulate the musculoskeletal properties of a seagull. Optimal wing configurations (joint values) were evaluated for a variety of flight regimes, and highlight the benefits associated with morphing. However, no mechanism was proposed and no comments were made on how morphing could be achieved [37].

To facilitate a bird perch maneuver, a specialized poly-morphing wing was developed for a small UAV. The wing is divided into two parts, and inboard and outboard section. Both wing sections are capable of variable sweep with the inboard section controlled by a four-bar linkage, and the outboard section controlled via a cable and pulley system. A separate mechanism controls the twist of the entire wing so that the wings can pitch to very high angles-of-attack for the perching maneuver. The outboard wing is also designed to fold under the inboard wing section, similar to a bird stowing its wings. A small kinematic prototype was developed, but only the wing spars were actuated as there was no physical wing to test for folding capabilities [38].

Finally, a concept for a morphing winglet was recently presented. The Morphlet is a morphing winglet concept that utilizes reoccurring morphing modules at the distal wing region to control the shape of a winglet. Each module was initially designed to be capable of altering the span (spar), taper, cant and twist angles. In the work presented, a two-module winglet with twist and cant variation was optimized for a variety of flight regimes [39]. Through discussions with the paper's lead author, the Morphlet was to remain a concept as no mechanism has been proposed to facilitate morphing. This is the only known example of a morphing wing system that had been developed in a modular fashion, in which each module possess specific morphing degrees-of-freedom.

\subsubsection{Other Morphing Wings}

Other types of morphing wings have been proposed that can not be categorized in terms of specific wing-level morphing degrees-of-freedom. For example, a compliant cellular truss was designed to 
facilitate large-scale, smooth morphing maneuvers. Instead of discrete wing sections with specific morphing degrees-of-freedom, the truss can actuate in any sensible manner to morph to a more optimal configuration. A bench model of the tendon-actuated truss was developed for structural testing and preliminary designs shown similar weights of the truss compared to a traditional stiffened wing. Possible aircraft skin systems were also given [40].

A small inflatable wing with airfoil shape control was developed for compact package and deployment. The inflatable wing is flexible and consists of a series of span-wise tubes that shape the wing. Through the use of piezoelectric actuators, the shape of airfoil is controlled by flattening bumps on the wing surface. A flight ready prototype was developed and bench testing of piezoelectric actuators was performed [41].

Inoyama et al. $[42,43]$ presented an algorithm which generates planar kinematic trusses for wing morphing. In their work, the presented algorithm determines an optimal kinematic truss configuration that facilitates a specific shape-morphing maneuver under static load and other constraints. The algorithm begins with a rectangular truss, and progressively replaces static truss members with ones that are either extensible or retractable, or simply removes truss members. An optimal kinematic truss configuration is thus generated that morphs the new wing truss from the original rectangular shape to some other predefined shape. Several examples are presented and compared as different constraints sets are imposed in the algorithm, thus the algorithm represents a development platform in which the morphing wing is developed to meet specific morphing requirements.

The final and perhaps most unique example of a morphing aircraft is the multi-role aircraft under current development by Lockheed Martin. The morphing aircraft can assume two distinct configurations that drastically alters the aircraft planform wing profile. The bi-fold wing has two joint sections aligned with the free stream flow that changes the wing from a high aspect ratio, low wing loiter configuration aircraft, to a small aspect ratio, high wing dash configuration aircraft. When assuming the loiter configuration, the wing is fully extended. When assuming the dash configuration, the inboard section of the wing rotates and rests against the aircraft body and the outboard wing section rotates in the opposite direction to maintain a level wing. Much research 
on the wing has been performed including ground testing of the bench prototype wing for seamless skin development [44], critical load analysis [45], and wind tunnel testing and validation [46].

\subsection{Actuation Methods for Wing-Level Morphing}

In the previous section, the literature was surveyed and morphing wing concepts, designs and prototypes were presented. Each work cited had a short description of the actuation method proposed to achieved the desired morphing maneuver. In this section, the actuation methods for the morphing methods surveyed in the literature are briefly compared to highlight the similarities and differences in implementing morphing wings. First, the single degree-of-freedom morphing methods are compared, followed by the poly-morphing methods. Lastly, a look at mechanical truss systems are compared.

\subsubsection{Single Degree-of-Freedom Mechanisms}

\subsubsection{Sweep-Morphing Wing Mechanisms}

For the wings that are capable of sweep-morphing, the differences in mechanisms lie in the style in which sweep-morphing occurs. In general, sweep-morphing can be achieved either by a shearing motion or a rotational motion about an axis perpendicular to the aircraft yaw axis. From the literature surveyed, rotational sweeping wings are more prevalent than those that shear as complex skin systems must be used for wings that shear. A sweep-morphing wing that is rotates about a fixed axis however, will in general, rotate a solid wing section, with specialized cutouts, or other measures taken to maintain a suitable aerodynamic surface; the F14 Tomcat is an example of this design, whereas the MFX-1 uses flexible skins $[13,14]$. The mechanism by which sweep-morphing is achieved can be a simple rotational joint [15], or through a four-bar linkage situated within the body of the aircraft $[11,13,14,38]$. Morphing wings that sweep via a shear motion have airfoil sections that maintain a consistent orientation to the free stream flow throughout the morphing maneuver, and the example from the literature uses a cellular truss to achieve this specific motion [12]. 


\subsubsection{Cant-Morphing Wing Mechanisms}

Cant-morphing, unlike sweep-morphing, is achieved solely through the rotation of a wing section about and axis that is parallel, or closely parallel, to the free stream flow. From the surveyed literature, shear motion has not be considered for a cant-morphing wing, thus all wings are in general, solid wing sections. To achieve cant-morphing maneuvers a single rotational joint has been primarily used for prototype development. For instance, the inboard section of the gull-

wing $[16,18]$, as well as all wing sections of the manta ray prototype [21] use rotary joints to achieve cant motion. The morphing aircraft by Lockheed Martin, although not specifically a cant morphing wing, actuates both wing sections in a similar manner to that of a cant-morphing wing, as the rotational joint axes are parallel to the free stream flow, and are used to morph the aircraft from one state to the next. The only instance of cant-morphing driven by a four-bar linkage was implemented for the outboard wing sections of the variable-gull wing. Here, the four-bar mechanism stowed within the body drives a slender rod that spans the length of the inboard wing section, below the wing, actuating the outboard cant angle $[16,18]$.

\subsubsection{Twist-Morphing Wing Mechanisms}

From the three rotational morphing degrees-of-freedom, twist-morphing is unique as it represents a torsional, or axial rotation of a flexible wing, or solid wing segments. For twist-morphing, a torque rod passing through the wing, usually through the 1/4-chord line, is used. Examples include the Morpheus [11], the UAV from the University of Florida [24-26], the UAV designed for perching maneuvers [28] and the elevon-replacing flexible twist-morphing wing [27]. Other methods have also been proposed including the use of SMA actuators [23]. A specialized four-bar linkage was also proposed to control the wing twist of a perching bio-inspired UAV [38].

\subsubsection{Span-Morphing Wing Mechanisms}

Span-morphing mechanisms are the easiest to categorize with respect to the mechanism due to the extensible nature of the morphing maneuver. Any prismatic-style (linear) actuator is capable of 
altering the span of the wing. Unsurprisingly, the majority of the span-morphing wings surveyed use some form of a linear mechanism, including using a track-guided scissor mechanism [35], a pneumatic telescoping rod [29,30], and a rack and pinion mechanism [33,34]. The exception to this is the expandable wing which uses a four-bar linkage to actuate the wing. The main difference with this particular prototype is span-morphing is achieved via rotating the outboard wing section from a stowed, to extended position [36]. No actuation method was listed for the span-morphing ballistic missile project, however the outboard wing section is stowed within the inboard fixed wing, and extends span-wise when morphing is required [31,32].

\subsubsection{Poly-Morphing Wing Mechanisms}

For single degree-of-freedom wings, there are some similarities in the methods used to actuate the wing. The mechanisms used to drive poly-morphing wings however are expected to be very different from one project to the next as each has been designed to achieve a specific set of morphing degreeof-freedom. As mentioned, some concepts and prototypes have multiple morphing capabilities, however the additional morphing degree-of-freedom is usually twist morphing of a flexible outboard wing section for control purposes $[11,15-18]$. The NextGen MFX-1 uses a cellular truss to alter the sweep of the wing, while simultaneously controlling the root airfoil chord length, thus altering the wing taper and aspect ratio $[13,14]$. Of the literature surveyed, the small bio-inspired UAV for perching is the only poly-morphing wing-capable two wing-level morphing degrees-of-freedom, and not including twist-morphing of a flexible wing section for control surface replacement-with a detailed description of the actuation method. Here, separate four-bar linkages control the sweep and twist of the wing independently. Other poly-morphing wing projects do not have a description of the mechanism that provides morphing.

\subsection{Summary of Morphing Wing Technology Surveyed}

In summary, a wide variety of morphing wings are present in the literature. While wing is designed to address specific morphing requirements, in each and every case, the proposed design is only 
capable of providing the wing with the particular morphing degree(s)-of-freedom being studied. The exceptions to this are the compliant truss wing [40], and the Morphlet project [39]. These projects exhibit the ability to study individual or combined morphing degrees-of-freedom without the need to redesign the wing. Unfortunately, as far as the author knows, the compliant truss wing has only a single bench-scale truss section for testing, and no working prototype of the Morphlet was developed. In addition to this, from the literature surveyed, the algorithm presented by Inoyama et al. $[42,43]$ is the only example in which a development platform has been attempted. The presented algorithm was applied to a variety of desired wing shapes, and with varying constraints, however with each iteration a completely new mechanism is generated, albeit of a similar style.

The goals of this dissertation outlined in Section 1.3 aims to address the above issues by not only providing a development platform for which a morphing wing can easily be generated, but also one that allows for a variety of morphing degrees-of-freedom to be utilized, either individual or in combination. In the following section, overviews of truss mechanisms and reconfigurable parallel robots are given as these mechanisms are used as basis for the development of the modular morphing wing system. The remaining chapters of this dissertation are dedicated to providing the details of the conceptual design and development of a modular morphing wing system.

\subsection{Mechanism Overview}

At their core, mechanisms can be categorized as being serial or parallel. Serial mechanisms and robots consist of a series of alternating structural links and controllable kinematic joints, terminating at an end effector which provides the means to do useful work. Parallel mechanisms and robots on the other hand consist of single or multiple kinematic loops. These loops consist of alternating structural links and kinematic joints, however, unlike their serial counterparts, not all of the joints within the loop are controllable. In fact, there need not be any controllable joints within a given loop. Recently, a combination of serial and parallel mechanisms have lead to the development of variable geometry truss mechanisms (VGTM). Truss-based mechanisms inherit properties from both the serial and parallel mechanisms from which they are derived, making them more complex 
than serial and parallel mechanisms, yet at the same time, much more versatile.

\subsubsection{Mechanism Style for Wing Morphing}

In Section 2.2 above, wing morphing mechanisms were grouped according to the degree(s)-offreedom of the wing, however, the mechanisms themselves can be categorized as being either serial, parallel or truss based.

Due to the weight and associative torque penalties of placing joints sequentially along the wing span, there are few morphing wings that can definitively be categorized as being serial in nature. Morphing wings such as the University of Florida's variable-sweep wing [15], the developed prototype manta ray wing [21], the pneumatic telescoping wing [29,30], the span-morphing MAV from the Universidade da Beira Interior [33], and the multi-role aircraft developed by Lockheed Martin [44], have each been developed using primarily serial style mechanisms, in which the inboard motors drive the mass of the wing and any subsequent outboard motor. Once again, the additional motor weight and torque must be considered in the design of these wings.

Parallel-style mechanisms have been proposed for wing morphing, primarily in the form of fourbar linkages. For instance, the Morpheus aircraft [11], the variable gull-wing aircraft from the University of Florida [16], the span-morphing aircraft with an expanding scissor mechanism [35], the expandable wing aircraft from Konkuk University [36], and the poly-morphing perching aircraft from the University of Maryland [38] all use four-bar linkages to drive one, or all of the joints within the wing.

The final category of wing mechanisms are those that utilize a truss for morphing, with each implementation being vastly different than the others. For instance, the wing concept by Bharti et al. [12] uses a single degree-of-freedom cellular truss to provide a shear-type sweep motion, whereas the manta ray wing concept (not prototype) uses a tensegrity truss for cant-morphing [21]. The planar cellular truss in the NextGen MFX-1, as mentioned above, is capable of sweep and taper/aspect ratio morphing. Large scale morphing is achievable with the proposed compliant truss [40]. These wings all use a modular cell structure within the mechanism, where cell modules 
are repeated throughout the mechanism. This feature is beneficial from a development perspective, and is a feature of the modular morphing wing developed in this dissertation. The final example of a truss-based wing is the algorithm presented by Inoyama et al. [42,43] which generates kinematic trusses for planar morphing maneuvers, though the resultant trusses are not modular.

\subsubsection{Truss Mechanism Overview}

Truss mechanisms, as mentioned, are derived from a combination of serial and parallel-style mechanisms. Architecturally, a truss mechanism is a sequential stacking of parallel-style mechanisms to form an open loop serial-style mechanism. Table 2.1 presents a relative comparison of truss mechanisms against their serial and parallel mechanism parents. The VGTM type inherits positive attributes from both serial and parallel mechanisms. For example, the payload-to-weight ratio of serial mechanisms tends to be very low. This is contrast to parallel mechanisms which generally have very high payload-to-weight rations. For a VGTM, the truss-structure of the mechanism has a higher stiffness than the structure of a serial robot, but not as high as that of an individual parallel robot. In general, a VGTM inherits positive qualities from serial and parallel mechanisms. The exception to this are the inherited complex kinematics.

Table 2.1: Relative comparison of mechanism archetypes

\begin{tabular}{llll}
\hline Parameter & Serial Mechanism & Parallel Mechanism & VGTM \\
\hline Forward kinematics & Simple & Complex & Complex \\
Inverse kinematics & Complex & Simple & Complex \\
Workspace & Large & Small & Large \\
Payload-to-weight ratio & Small & Very large & Large \\
Force transmission & Low & Very high & High \\
Dexterity & High & Very low & High \\
Repeatability & Low & Very high & High \\
Stiffness & Very low & Very high & High \\
\hline
\end{tabular}


While truss mechanisms generally have complicated kinematics, they inherit positive qualities from both serial and parallel mechanisms. From serial mechanisms, they inherit large workspaces and dexterity, while inheriting high payload-to-weight ratios, force transmission and repeatability from parallel mechanisms. The mechanisms qualities listed in Table 2.1 reflect general capabilities of truss-based mechanisms, but a more detailed description is given below with respect to requirements of a morphing wing system.

\section{Kinematics Requirements}

A morphing wing system capable of large-scale geometric changes to the wing shape requires a robust mechanism to provide the necessary kinematic motion. The position of the wing tip in a system as such might experience large displacements with respect to the body of the aircraft, and many mechanisms are certainly capable of providing such motion. For wing morphing, the internal position and orientation of the mechanism is equally important to the wing tip position and orientation. This requirement is generally not a concern for traditional mechanisms, however, truss-based mechanisms provide the capabilities to individually control segments of the mechanism. Specifically, a modular system allows for self-contained, individual kinematic requirements that are tailored specifically to needs required in that area of the wing. For instance, sweep and cant morphing capabilities might be required near the wing root, whereas span and twist morphing might not be. Conversely, an area of the wing might require all four morphing degrees-of-freedom, whereas others might require only

a single morphing degree-of-freedom. A modular, reconfigurable wing morphing system can be designed to individually or simultaneously provide the four morphing degrees-of-freedom. Thus the kinematic requirements at specific span-wise locations can individually addressed in a modular fashion, providing only the necessary motions to each area of the wing, while eliminating the need to develop specific kinematic systems for each wing morphing aircraft.

\section{Structural Requirements}

A morphing wing will experience a range of load profiles throughout its mission, which must be sustained by the mechanism that provides morphing. In situations where the wing is 
not required to morph, controllable truss members within the system are locked, resulting in a traditional truss. During a morphing maneuver, however, the controllable members are unlocked and actuated according to the particular morphing requirements. The actuators must not only be able to move the dry-weight of the wing, but also do so adequately under loading conditions imposed by the aerodynamic loads exerted on the wing during flight. The very nature of truss-based mechanisms is that they simultaneously provide the kinematic requirements of a mechanism with the structural capabilities of a truss. In addition to this, truss structures are naturally modular, as are truss-based mechanisms, thus they can easily be utilized in the conceptual development of a modular wing morphing mechanism.

\section{Volume Requirements}

Finally, there is a significant constraint on mechanism sizing as the profile of a wing restricts what can used to actuate a morphing mechanism. In a serial-style robot, very large actuators and links are required at the base of the mechanism in order to support the anticipated load. This physical requirement is mitigated with the use of parallel-style mechanisms, and thus by extension, truss-based mechanisms. The sizing of the truss-members at the base of the wing will naturally be larger to support the bending moments imposed by lift and drag distribution along the wing, however a truss can easily be adapted to fit within the structural space originally allocated for the wing box. This is also the case with a truss-based mechanism, and allows for a design to fit entirely within the bound volume of the wing.

The kinematic, structural and volume requirements listed above must each be addressed for the development of a truss mechanism for wing morphing. The structural requirements are beyond the scope of this dissertation, including the weight implications of removing the structural members of the wing box and replacing them with a kinematic truss. However the kinematic, and by extension, volume requirements are addressed here. What is evident and has previously been mentioned, is that the modules of a truss mechanism are in fact, individual parallel-style mechanisms. This modularity allows for the truss mechanism to be analyzed at the module level as opposed to the system level as a whole. In addition to modularity, the proposed morphing wing systems is 
reconfigurable. Hence, the individual wing modules are also reconfigurable. As the wing modules consist of parallel mechanisms, a review of reconfigurable parallel mechanisms is given below.

\subsubsection{Reconfigurable Parallel-Style Mechanisms}

Traditionally, reconfiguration of a parallel mechanism is achieved with the desired goal of altering the motion characteristics of the moving platform or end effector. An example of this is reconfiguration which changes the motion profile and subsequent workspace of limited mobility mechanisms (mechanisms with less than six controllable degrees-of-freedom for spatial motion, and those with less than three for planar motion). This is generally achieved by switching joints within any of the branches in the mechanical system. Instead of reconfiguring the joints within the system, the shape of the reachable workspace can be changed by altering the size of the links within any of the branches, or by altering their connection points within the system. Alternatively, the shape of the workspace and its kinematic qualities can be enhanced by means of redundancy within any of the branches. Kinematic qualities here can be conditioning of the Jacobian matrix or singularity avoidance, as an example. Reconfiguring joints and reconfiguring links are mutually exclusive, but can also be used in combination to further enhance the reconfiguration capabilities of a parallel mechanism or robot.

Parallel robots, specifically, are attractive candidates for reconfiguration due to the modular nature of their branch systems where reconfiguration can be classified as being geometric, topological or a combination of the two (classified as group reconfiguration) [47]. Geometric reconfiguration is the process of adjusting the size or orientation of branch components without rearranging their sequence. An example of a reconfigurable parallel robot capable of geometric reconfiguration is the reconfigurable tricept machine tool developed by Bi and Wang [48]. A reconfigurable parallel robot system was proposed in which standard components are used in an optimization algorithm to create different sized tricept robots. A new reconfigurable Hooke (universal) joint was proposed for the reconfiguration of parallel robots. The specialized Hooke joint allows for one axis to be oriented at orthogonal angles with respect to the initial configuration, thus altering the motion profile of 
the joint itself. Reconfiguring the joint alters the motion profile of the parallel robot $[49,50]$.

Redundancy within the robot structure also allows for geometric reconfiguration. Kotlarski et al. [51] presented a parallel robot capable of reconfiguration via redundancy within the system. Instead of a fixed position on the base, one branch is connected to a prismatic joint that is passively activated to alter the geometry of the parallel robot. As the resultant workspace is altered after reconfiguration, the robot is capable of singularity avoidance. Spatial reconfigurable robots have also been presented recently. Bi and Kang [52] presented a reconfigurable Gough-Stewart platform. Offline adjustments to the connection points of the branches on the base and platform, as well as other geometric features allow various permutations of the traditional Gough-Stewart platform to be assumed. In their work, they explore the kinematic and structural stiffness properties associated with reconfiguration. A reconfigurable Stewart platform for contour generation was presented [53]. More recently, a reconfigurable tricept robot was also presented [54].

Topological reconfiguration is more complex and is associated with the re-sequencing of joints within the branch structure, usually to achieve a different motion profile for the platform. For instance, Dash et al. [55] presented a reconfigurable system in which multiple actuation modules are combined with link modules to assemble different parallel robot configurations. By altering the number of branches and actuation modules within each branch, the topology of the robot is drastically altered. Xi et al. [56] presented a reconfigurable parallel-robot with varying platform degrees-of-freedom and detachable serial arms. The robot can reconfigured to configurations with mobility ranging from three to six by detaching arms from platform, and locking joints. The detached arms remain active, and in fact, gain a degree-of-freedom to be used in unison with the reconfigured parallel robot. Other branch configurations for an on-the-fly reconfigurable parallel robot were also studied [57]. A new fully reconfigurable, isomorphic parallel robot was presented. The presented robot can assume platform mobility ranging from three to six, however when reconfiguring to limited mobility modes, all branches remain fixed to the base and platform. As each branch is a candidate for reconfiguration, isomorphism within the reconfiguration are present, further expanding the capabilities of the robot $[58,59]$. This type of reconfiguration uses reconfigurable 
joints that can assume active or passive states, similar to work presented in [60]. Redundancy can also be used, as seen in the topological reconfigurable 4-branch parallel robot [61].

Group morphing as mentioned is a combination of geometric and topological reconfiguration and is seen in advanced systems such as variable geometry truss mechanisms (VGTM). The original modular morphing wing module is one such example as categorized in the paper presented Xi et al.w [47]. Multiple level of reconfiguration of the wing allow for unique wing shapes [62].

Reconfiguration can be performed automatically, or require external assistance. Reconfigurable parallel robots built with redundancy, in general, can be reconfigured automatically as shown with $[51,52]$. Other robots require external assistance for reconfiguration as the robot configuration is assembled from a cache of link and joint modules [48,61]. However, in these cases, the mobility of the system remains fixed. Despite the obvious advantages to reconfiguration, the achievable tasks of the mechanism are limited to the initial mobility of the mechanism itself. For example, a task requiring independent planar positioning and orientation, i.e. three degrees-of-freedom, would require a mechanism with three controllable degrees-of-freedom, or a mobility of three. If an additional degree-of-freedom was added to the task requirements, then a reconfigurable parallel mechanism must have the ability to add a degree-of-freedom and assume a configuration in which the mechanism mobility matches the degrees-of-freedom of the task. The system presented by Dash et al. can address these task-based reconfiguration requirements, however reconfiguration requires external assistance [55]. Another example is the task-based reconfigurable robotic system has also been proposed using a variety of robot structures [63]. From the parallel robots listed, [56,57,59,64] are the only one in which a change in mobility is achievable via reconfiguration without the need for external assistance. These are examples of reconfigurable parallel robots in which task-based mobility requirements are built into the reconfiguration system.

\subsection{Summary}

In this chapter, pertinent works within the literature were surveyed and presented. First, morphing wing concepts, designs and prototypes were presented. Focusing on wing-level morphing, concepts 
and designs that provide individual sweep, cant, twist and span morphing were shown, followed by poly-morphing wings as well as specialized morphing wings. After, the mechanisms used for morphing were categorized according the style of mechanism. Here, wing morphing mechanisms were categorized as being serial, parallel and truss-based. With morphing wing technology introduced, an overview of mechanisms was presented, including a focus on truss-based mechanisms. Requirements for truss-based wing morphing mechanisms were summarized, and the concepts of modularity and reconfigurability were established for the presented morphing wing system. This was followed by a survey of reconfigurable parallel mechanisms and robots. 


\section{Chapter 3}

\section{Wing Module Discretization}

In this chapter, a novel discretization method is presented that determines the number and associative spacing of wing modules required to perform a morphing maneuver. First, an overview of the placement of kinematic joints within current morphing wing systems is given, including a discussion on modular morphing wing requirements. Next, the discretization method is presented, which determines the minimum number of wing modules required, and their associative spacing to sufficiently emulate a known, desirable wing shape. Included are details of the various steps within the algorithm. With the algorithm established, an overview of differential curve geometry is given as concepts from the Frenet-Serret and Parallel-Transport curve framing methods are used within the discretization algorithm. In addition to this, a new curve framing method is developed specifically for the discretization of a modular morphing wing. A case study is then presented that

shows how the discretization method is used to determine the number and associative spacing of wing modules for a hypothetical wing and morphing maneuver. Finally, a summary of the chapter is given.

\subsection{Morphing Wing System Overview}

A morphing wing is capable of altering its shape in some way for the purpose of improved flight performance. To facilitate morphing, mechanical joints are placed at various stations along the wing, and this provides the wing with kinematic motion capabilities. The number and location of 
these joints obviously has a direct impact on morphing, but beyond that, the number and location of joints defines the limit on which potential performance gains a morphing aircraft can achieve. As such, joint location is an important aspect of wing morphing, however as will be shown, it tends to be overlooked when a morphing wing is developed. In this section, an overview on joint placement within current morphing wing systems is given. Following this, a discussion is given on modular morphing wing systems in terms of implementation and performance, and requirements that are imposed are highlighted.

\subsubsection{Joint Placement in Morphing Wing Mechanisms}

As surveyed in Chapter 2, many different methods to morphing have been presented. From the projects surveyed, almost every morphing wing aircraft has a single joint placed at the root of the wing which alters the configuration of the remainder of the wing as a whole [11-14]. The obvious exception to having a joint at the wing root are those projects focusing on wingtip morphing such as $[19,20,23,27,39]$.

Those morphing wings that do have additional joints along the wing generally have one positioned at or near the mid-wing (quarter-span) position [15-19,28,37,38]. The placement of the second joint in these cases for the most part is entirely arbitrary. Selecting the mid-wing position may or may not offer any benefit over positioning the additional joint elsewhere on the wing.

One unique exception to arbitrary joint placement are the family of morphing aircraft developed at the University of Florida. These morphing aircraft are bio-inspired as the skeletal structure of a seagull was used as a reference for joint placement along the wing [15-18,37]. Here, the aircraft were design to mimic the physical features of a seagull in flight for a variety of flight profiles.

Without exception, a morphing wing will have a profound impact on the performance of an aircraft when the wing configuration is altered. Some research has been performed on the effects of joint location on performance. Ameri et al. [20] compared the dynamic performance of a morphing wing with different sized active winglets designed to replace conventional aircraft control surfaces. Stanford et al. [65] from the University of Florida used performance requirements to optimized 
the roll rate and lift-to-drag ratio for a twist-morphing aircraft by altering the connection points of a torque rod along the wing. They show that through an optimization algorithm, altering the torque rod configuration and connection points can not only improve the roll rate and lift-to-drag ratio, but can also result in a decrease in these indices as compared to the original torque rod configuration. Inoyama et al. [42,43] used a topology optimization algorithm to select actuator type and placement of a planar morphing wing. In their paper, a genetic algorithm is used to create a planar morphing wing that is capable of morphing between a rectangular planform shape and a predetermined morphed wing shape.

\subsubsection{Requirements of Modular Morphing Wings}

As seen from many of the projects surveyed in Section 3.1.1, the arbitrary placement of joints within the morphing wing system can limit the potential performance gains that morphing wings grant. Similarly, designing a morphing wing system first, and then analyzing its potential also restricts the performance gains that are attainable through wing morphing. On the other hand, using some type of performance parameter to influence the design of the morphing wing systems allows the wing to provide near-optimal performance.

If a desirable wing shape for a particular flight regime is known, then a morphing wing must be developed to sufficiently emulate that desirable reference wing. The goal is to develop a modular morphing mechanism that provides the wing with enough degrees-of-freedom to sufficiently emulate the reference wing shape. As the wing is expected to require different morphing capabilities at different regions of the wing, then the morphing wing module must be designed to achieve all expected morphing degrees-of-freedom. This not only allows for modules to be used at any portion of the wing, but it also allows for any number of modules to be used. The challenge is then to determine how many modules are needed, and where they should be placed along the wing span. If the entire wing is to be made of morphing wing modules, then their sizing can be adjusted according to the complexity of the reference wing. Wing regions that are predominantly straight and untwisted requires fewer degrees-of-freedom to adequately describe the wing, thus fewer, longer 
modules are needed. Regions of the wing that are not straight and contain significant amount of twisting or bending are geometrically complex, thus a greater number of tightly spaced modules are required to successfully describe the wing degrees-of-freedom. Implementing the morphing wing system in this fashion allows for the morphing wing to better emulate the wing from which its design is required to emulate. The advantage to this approach is that the developed morphing wing will also emulate the performance of the desirable reference wing.

It is noted that with any morphing wing, performance losses are expected due to morphing itself and the implemented morphing mechanism. Thus a morphing wing is capable of near-optimal flight performance for particular flight regimes. For a modular morphing wing, losses occur due to discretized wing sections, however, since a desirable wing shape is used to influence the morphing wing configuration, then the performance loses can be minimized. Using these requirements, a discretization method can be developed to determine the number of wing modules required, and their associative span-wise distribution for a particular flight regime. In the following section, the discretization algorithm is introduced and details on the various steps are given.

\subsection{Discretization Method}

The following discretization method presented addresses the first objective of this dissertation $[58,66]$. The purpose of the discretization method and the developed algorithm is to determine the minimum number of morphing wing modules required to achieve a desired flight performance associated with a particular flight regime or requirement. This discretizes the otherwise continuous wing into a discrete set of modules with specific module sizes. In the next section, the algorithm is introduced, after which details of each step is given.

\subsubsection{Discretization Algorithm}

The discretization algorithm is a two step curve fitting process that alternates between the geometric discretization of the wing into morphing modules, and the evaluation of the morphing wing flight performance. First, a reference wing is defined in regards to its wing curve, then beginning with two, 


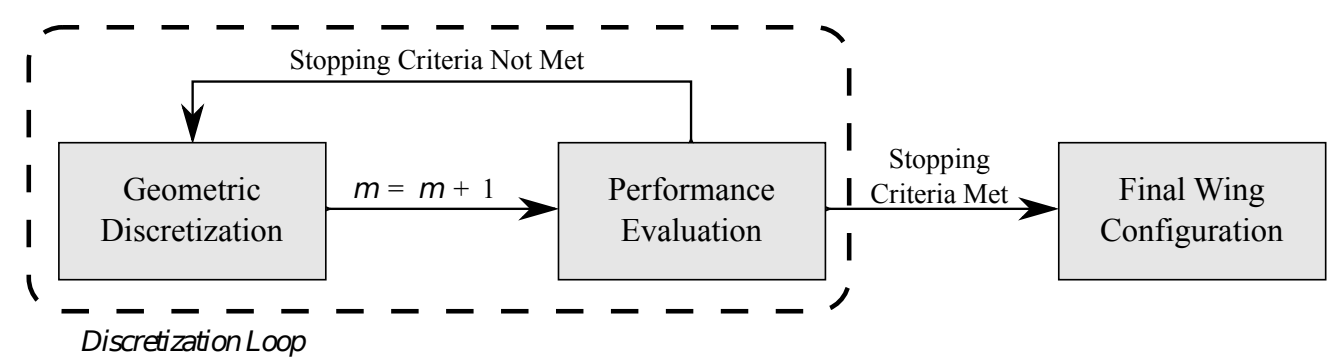

Figure 3.1: Discretization algorithm

and incrementally increasing the number of modules, the performance of the current morphing wing configuration is recursively checked for an acceptable stopping criteria. A flow chart depicting the steps of the discretization algorithm is shown in Fig. 3.1. The steps of the discretization algorithm are as follows:

1. Discretize the wing into $m$ modules. Module spacing is determined by equally distributing the wing curvature and wing torsion amongst the $m$ modules.

2. Calculate the relevant performance parameter(s) of the discretized wing. If the performance parameters area acceptable, are no longer changing as modules are added, or the performance is decreasing significantly, then stop, otherwise, go to Step 3.

3. If $m=m_{\max }$, then stop, otherwise, increase the number of modules $m=m+1$ and return to Step 1.

An example of the discretized algorithm is depicted in Fig. 3.2 where a hypothetical wing curve is shown. Also depicted are three wings that have been generated using the discretization algorithm. The curve used describes a wing shape similar to that of a bird gliding close to the ground. The first wing, Fig. 3.2(a), is discretized into two modules, and as seen, does not accurately emulate the reference wing curve. The second wing, Fig. 3.2(b), is discretized into five modules. With the additional modules, we see that the reference curve is better emulated by the discretized wing. We also note that as additional modules are added, they are clustered within the central region of the wing. This region is the most geometrically complex as there wing is highly curved. The final wing shown in Fig. 3.2(a), is discretized into 10 modules. Here, there is very little difference 


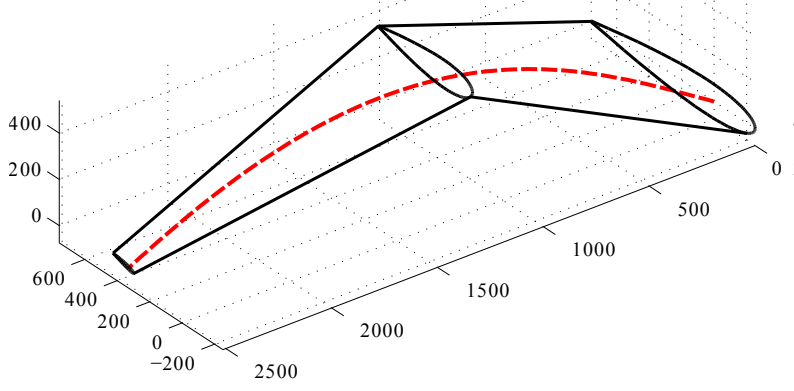

(a) 2 module wing

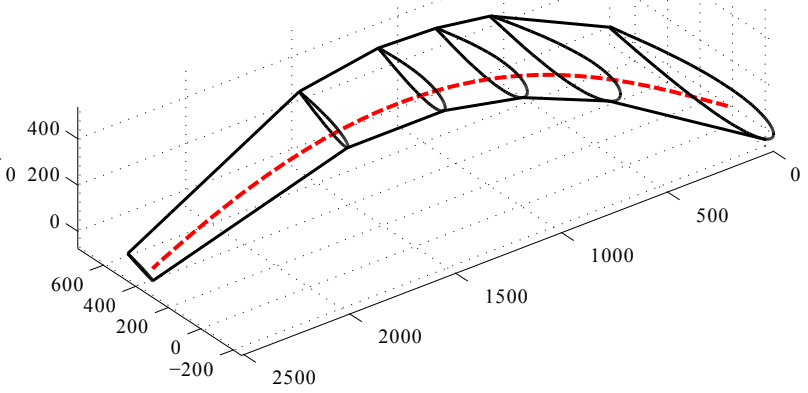

(b) 5 module wing

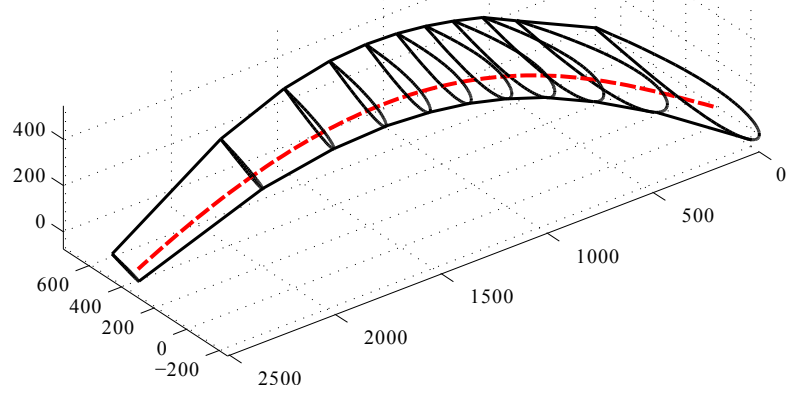

(c) 10 module wing

Figure 3.2: Discretization algorithm example of a wing curve defining a bird gliding close to the ground

between the original wing curve and the discretized wing. Again, additional modules are clustered within the central wing region, and those near the wing root and wing tip are continually becoming shorter in length. This trend will obviously continue as additional modules are added. Continuing to increase the number of modules will eventually result in the discretized morphing wing reaching a continuum.

Prior to detailing the steps of the algorithm in Fig. 3.1, some notes about the algorithm are given. First, the algorithm uses a previously determined or desired wing shape as a reference and distributes the modules of the morphing wing according to the geometry of the reference wing. Second, the algorithm is not intended for wing shape optimization or for shape rate control, rather it is used generate a modular morphing wing that is capable of performing a desired morphing maneuver from an initial straight and untwisted state. By using the reference wing curvature 
and twist distribution, the algorithm spaces modules to emulate the geometric features of the wing, not by simply dividing the span of the wing evenly amongst the modules. Thus, wing regions with large curvature and/or twist distribution have several, tightly spaced wing modules as compared to wing regions with small curvature and/or twist distribution in which fewer, longer wing modules are required. By distributing the modules as such, the developed modular morphing wing can sufficiently emulate the shape of the reference wing, and as such, provide the aircraft with near-optimal flight performance for the particular flight regime or requirement associated with the reference wing.

\subsubsection{Geometry Discretization}

The first step of the discretization algorithm is geometry discretization. This step within the discretization algorithm incrementally increase the number of modules within the current wing configuration. The main purpose of this step is to determine the spacing of the modules, so that the performance of the current wing configuration can be analyzed.

As mentioned, the modules are spaced according to the local geometric features of the reference wing for which the morphing wing is designed to mimic. The minimum number of modules that can be used is two. Using only a single module defeats the purpose of the reusability of the wing module, and does not grant the aircraft sufficient control of the wing shape. Thus the geometry discretization requires at least two modules to be used.

When the wing geometry is discretized, each module consists of a base and platform airfoil rib. The base airfoil of the first module is situated at the root of the wing, and the platform airfoil of the most outboard module is situated at the tip of the wing. The remaining modules within the interior region of the wing share common airfoil ribs between adjacent modules. The platform airfoil of one module acts as the base airfoil of the subsequent module, thus maintaining continuity along the wing. The local position of the interior airfoil ribs is what the geometry discretization portion of the algorithm achieves, and their respective spacing is dependent on the geometry of the reference wing. To accomplish the spacing previously mentioned, concepts from differential 
geometry are used, and are detailed in Section 3.3. With the module, and thus airfoil rib, spacing established, the current wing configuration's performance can then be analyzed.

\subsubsection{Performance Analysis}

With each successive iteration of the discretization loop, an additional module is added to the wing, thus altering the overall shape of the wetted wing surface. Thus the performance of the current morphing wing configuration must be evaluated. This represents the second step of the discretization algorithm. To find the minimum number of morphing wing modules, four criteria are used to stop the discretization loop:

1. The performance of the discretized wing has asymptotically approached a maximum attainable performance where the addition of modules does not change the performance. This maximum performance is equal to the reference wing performance.

2. The performance of the discretized wing is within an allowable user-defined tolerance of the reference wing performance and no additional modules are added to minimize the wing complexity.

3. The number of modules has reached the user-defined maximum allowable modules. The potential loss of performance is deemed acceptable in order to minimize the wing complexity.

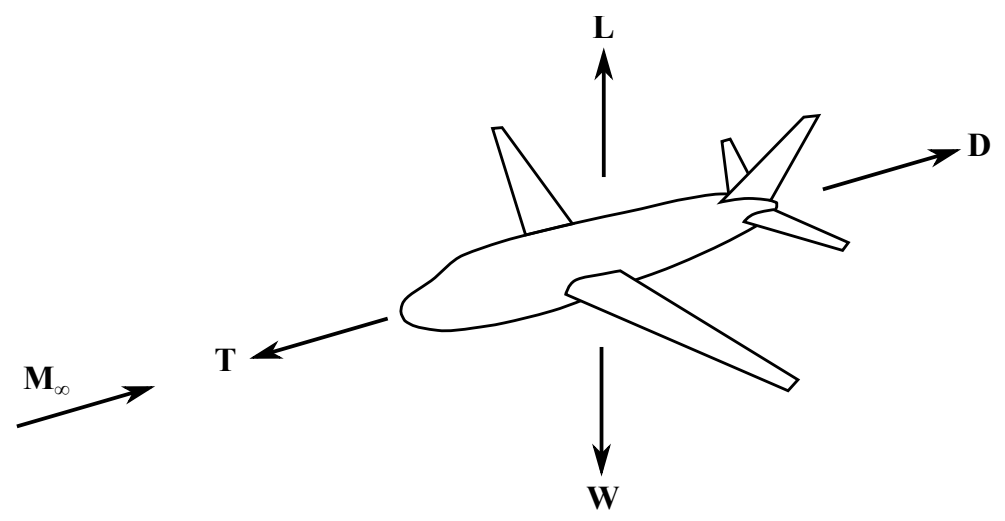

Figure 3.3: Aircraft forces in steady level flight 
The final performance of the morphing is obviously dependent on the number of modules used. As more modules are added, the variation in performance will diminish due to the similarity between the morphing wing configurations. The selection of the performance parameter itself will depend on the mission requirements. For instance, the lift-to-drag ratio is a fundamental parameter that affects the cruise performance of an aircraft. Other indices related to aircraft stability, accelerated flight or maneuvering can also be used depending on the mission requirements [8-10]. However in the case study, the lift-to-drag ratio, $C_{L} / C_{D}$, is used as a performance index. For steady level flight, lift is equal to the aircraft weight, and drag is equal to the aircraft thrust as shown in Fig. 3.3. A case study is shown to highlight the effectiveness of the discretization method and is shown in Section 3.5, however in the following section, the relevant differential curve geometry theories are outlined and then applied to achieve the geometry discretization step of the algorithm.

\subsection{Differential Curve Geometry}

In this section, highlights of different curve geometry are given as they pertain their use in the wing discretization algorithm. First, an introduction on curve framing is given, with a discussion on the Frenet-Serret and parallel-transport curve framing methods. Following this section, the method used to frame the wing curve is outlined.

\subsubsection{Curve Framing}

In differential geometry, curve framing is the mathematical description of a curve in space. It allows the position of the curve to be defined in $n$-space, and defines local geometric characteristics, such as the curve tangent, curvature, torsion, etc.

Although a curve can mathematically be described in a space of any dimension, for the purpose of the wing discretization algorithm, curves are limited to 3-space. Let $\mathbf{s}(\mathbf{t})$ be any 3 -space curve with a parameterization variable, $t$. The parameterization variable can be of any dimension, but it is common and advantageous to define $t$ such that the curve $\mathbf{s}(\mathbf{t})$ is arc length parameterized. In

other words, $t$ lies on the interval $[0,1]$, where $t=0$ and $t=1$ are representative of the beginning 


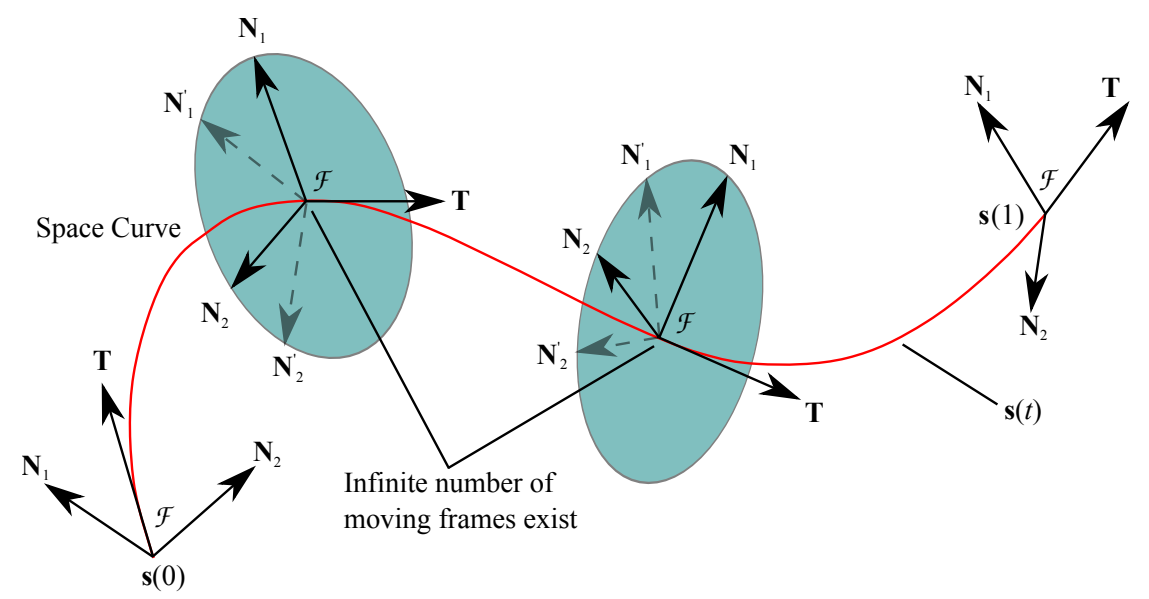

Figure 3.4: Space curve showing infinite number of curve frames

and end of the curve, respectively. The beginning and end points of the curve can be arbitrary, however, when analyzing a particular curve, a specific portion of the curve is generally meant to be analyzed. Thus, if the arc length of a curve section is $\AA$, then the arc length of an arc-length parameterized curve can be calculated

$$
\AA=\int_{0}^{1} \mathrm{~d} \mathbf{s}(t)=\int_{0}^{1} \sqrt{s_{x}^{2}(t)+s_{y}^{2}(t)+s_{y}^{2}(t)} \mathrm{d} t
$$

where $s_{x}(t), s_{y}(t), s_{z}(t)$ are the $x, y$, and $z$ components of the space curve. The beginning of the curve can, but does not necessarily originate at the origin when $t=0$.

If an arc length parameterized curve in 3 -space is defined by its $x, y$, and $z$ components, then the location of the curve at any $0 \leq t \leq 1$ is known. However, what is not known is the local orientation of the curve. In order to describe the curve orientation, curve framing methods are used. Basically, a curve frame is a moving coordinate frame, $\mathcal{F}$, whose origin lies on the curve, and at any point on the curve, describes the local orientation of the curve as seen in Fig. 3.4. If one axis of the moving frame is required to remain locally tangent to the space curve, then the remaining two axes must be defined so that the curve orientation is consistently described by the moving frame. This challenge arises due to the fact that there are an infinite number of frames that can describe a curve as shown in Fig. 3.4. Because of this, methods were developed to consistently frame a curve. Two methods, the Frenet-Serret and parallel-transport framing methods are described below. 


\subsubsection{Frenet-Serret Frame}

The Frenet-Serret curve frame is a framing method discovered independently by Jean Frédéric Frenet in 1847, and Joseph Alfred Serret in 1851 and is named accordingly. A depiction of the frame is shown in Fig. 3.5, where the coordinate frame is defined by the tangent, $\mathbf{T}$, the binormal, $\mathbf{B}$, and the normal $\mathbf{N}$ vectors. If the curve is arc-length parameterized, then the coordinate frame components are defined as $[67]$

$$
\begin{gathered}
\mathbf{T}=\frac{\mathrm{d} \mathbf{s}}{\mathrm{d} t} \\
\mathbf{B}=\frac{\mathrm{d} \mathbf{T}}{\mathrm{d} t}\left\|\frac{\mathrm{d} \mathbf{T}}{\mathrm{d} t}\right\| \\
\mathbf{N}=\mathbf{T} \times \mathbf{B}
\end{gathered}
$$

The three basis vectors are related by

$$
\left\{\begin{array}{c}
\frac{\mathrm{d} \mathbf{T}}{\mathrm{d} s} \\
\frac{\mathrm{d} \mathbf{N}}{\mathrm{d} s} \\
\frac{\mathrm{d} \mathbf{B}}{\mathrm{d} s}
\end{array}\right\}=\left\|\mathbf{s}^{\prime}(t)\right\|\left[\begin{array}{ccc}
0 & \kappa(t) & 0 \\
-\kappa(t) & 0 & \tau(t) \\
0 & -\tau(t) & 0
\end{array}\right]\left\{\begin{array}{l}
\mathbf{T} \\
\mathbf{N} \\
\mathbf{B}
\end{array}\right\}
$$

where $\kappa(t)$ and $\tau(t)$ are the curvature and torsion of the space curve, and each are defined locally with respect to the curve parameterization variable, $t$. In this form, and as shown in Fig. 3.5, T, $\mathbf{N}$, and $\mathbf{B}$ are orthonormal basis vectors of the frame $\mathcal{F}$, inter-related by the curvature and torsion of the curve. Curvature is a tendency for the curve to deviated from the local tangent, $\mathbf{T}(\mathbf{t})$ where torsion is a measure of the twisting of the osculating plane, or the orientation of the normal and binormal basis vectors at any point $t$.

If $\mathbf{s}$ posses $C^{3}$ continuity, i.e. is thrice differentiable, and the curve velocity, $\mathbf{s}^{\prime}$, and curve acceleration, $\mathbf{s}^{\prime \prime}$ are linearly independent*, then the curvature and torsion of a Frenet-Serrent curve are respectively

$$
\kappa(t)=\frac{\left\|\mathbf{s}^{\prime}(t) \times \mathbf{s}^{\prime \prime}(t)\right\|}{\left[\mathbf{s}^{\prime}(t)\right]^{3 / 2}}
$$

${ }^{*}$ The curve velocity and acceleration are differentiated with respect to the curve parameterization, $t$, and are represented of the velocity and acceleration of the motion of a frame along the curve 


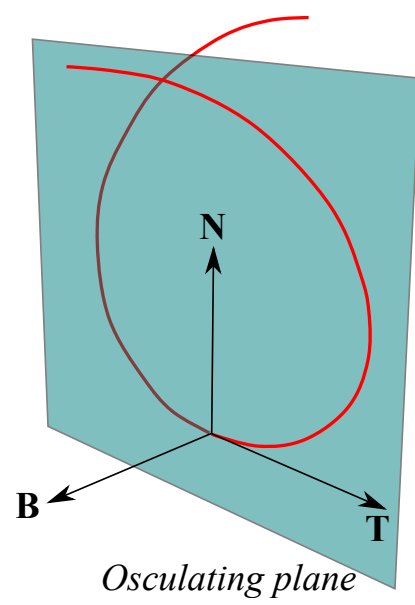

Figure 3.5: Frenet-Serret moving frame and osculating plane

and

$$
\tau(t)=\frac{\mathbf{s}^{\prime}(t) \times \mathbf{s}^{\prime \prime}(t) \cdot \mathbf{s}^{\prime \prime \prime}(t)}{\left\|\mathbf{s}^{\prime}(t) \times \mathbf{s}^{\prime \prime}(t)\right\|^{2}}
$$

We note that curvature and torsion are local geometric features of a curve, and are generally different at any $t$. A unique curve in which curvature and torsion are constant is a helix. In order for Eq. (3.6) and (3.7) to be valid at any $t$, a curve described by the Frenet-Serret formulae, Eq. (3.5) must be thrice differentiable, and must have linearly independent curve velocity and acceleration.

\subsubsection{Parallel-Transport Frame}

An alternative to the Frenet-Serret frame was proposed originally in 1975 by Bishop in which the normal development of a curve was used to describe a curve frame [67]. In this work, he described the new framing method which uses a relatively adapted parallel frame. Later Hanson and Ma referred to this method as parallel transport frame, which uses the properties of parallel vector fields to describe a curve frame. Basically, a parallel vector field with normal vectors fields $\mathbf{N}_{1}(t)$ and $\mathbf{N}_{2}(t)$ on a curve $\mathbf{s}(t)$ maintains a common orientation along the entire vector field. If we evaluate two points, $t_{i}$ and $t_{i+1}$, along the field, in the limit as $\delta t=t_{i+1}-t_{i} \rightarrow 0$, we see that $\mathbf{N}_{1, i} \rightarrow \mathbf{N}_{1, i+1}$ and $\mathbf{N}_{2, i} \rightarrow \mathbf{N}_{2, i+1}$. Thus, we can see that applied to curve framing, a parallel transport frame is parallely transported along a curve, defining a parallel vector field. An example 


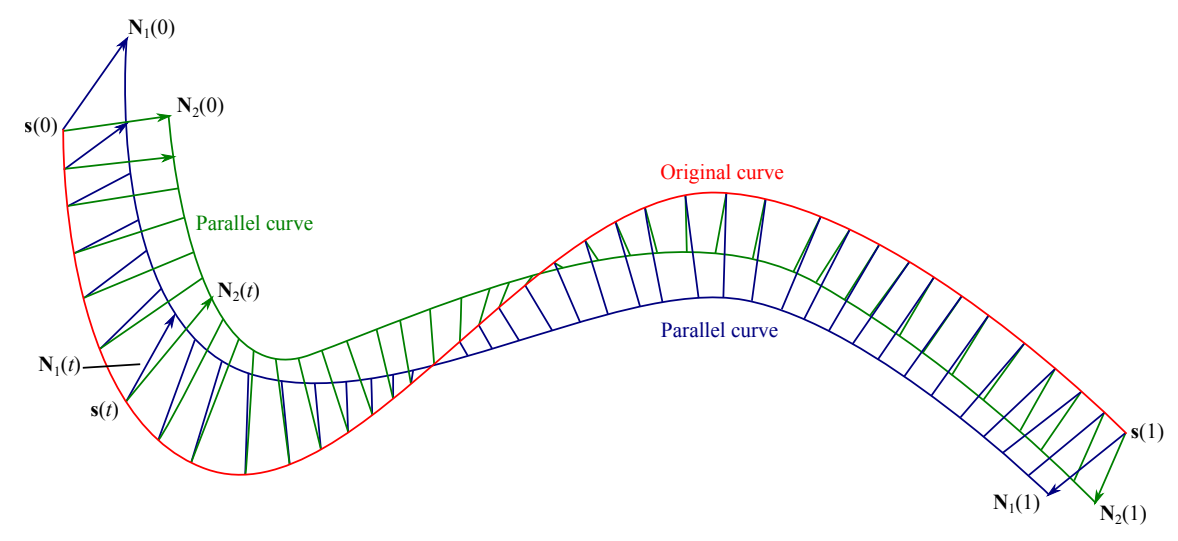

Figure 3.6: Parallel vector field

of the parallel vector field is shown in Fig. 3.6

The three basis vectors $\mathbf{T}, \mathbf{N}_{1}$ and $\mathbf{N}_{2}$ are related by

$$
\left\{\begin{array}{c}
\frac{\mathrm{d} \mathbf{T}}{\mathrm{d} s} \\
\frac{\mathrm{d} \mathbf{N}_{1}}{\mathrm{~d} s} \\
\frac{\mathrm{d} \mathbf{N}_{2}}{\mathrm{~d} s}
\end{array}\right\}=\left\|\mathbf{s}^{\prime}(t)\right\|\left[\begin{array}{ccc}
0 & k_{1}(t) & k_{2}(t) \\
-k_{1}(t) & 0 & 0 \\
-k_{2}(t) & 0 & 0
\end{array}\right]\left\{\begin{array}{c}
\mathbf{T} \\
\mathbf{N}_{1} \\
\mathbf{N}_{2}
\end{array}\right\}
$$

where for the parallel transport frame, $k_{1}$ and $k_{2}$ are parameters which are used to calculate the curvature and torsion by $[67]$

$$
\kappa(t)=\sqrt{k_{1}^{2}(t)+k_{2}^{2}(t)}
$$

and

$$
\tau(t)=-\frac{\mathrm{d} \theta(t)}{\mathrm{d} t}
$$

where

$$
\theta(t)=\arctan \left(\frac{k_{2}}{k_{1}}\right)
$$

Here, we see that the parameterization terms $k_{1}$ and $k_{2}$ are the two fundamental parameters that dictate the motion of the frame along the curve, and that $\theta$ is essentially a differential measure of the twisting of the vector field described by the moving frame. As such, the initial choice of $\theta$ can be arbitrarily, but also, additional spin can be given to the parallel transport frame to force the frame to arrive at a particular orientation at the end of the curve [68]. 


\subsubsection{Curve Frame Comparison}

The Frenet-Serret and parallel transport frames each describe the motion of a moving coordinate frame along a curve. However, there are instances in which the Frenet-Serret formulae fail where the parallel transport frame succeeds. An example of this phenomenon is the rooftop example shown in Fig. 3.7. Here, we see that at the tip of the rooftop, the Frenet-Serret frame (Fig. 3.7(a)) is undefined, and thus the moving frame is discontinuous at this point. Further, when the frame passes this inflection point, the coordinate frame does not maintain its orientation with respect to the initial portion of the curve; the binormal basis vector $\mathbf{B}$ initially pointing above the rooftop, has inflected and points below the rooftop at the end of the curve. The parallel transport frame (Fig. 3.7(b)) on the other hand is defined at all points of the curve, including the tip inflection point. Additionally, since the parallel transport frame is developed based on parallel vector fields, the orientation of the frame throughout the curve maintains a smooth transition, without a discontinuous switch in orientation as seen with the Frenet-Serret frame. It is for this reason, that the parallel transport approach to curve framing is used for the wing curve definition, however aspects of the Frenet-Serret frame are used. In the sections that follow, details of the wing curve are given.

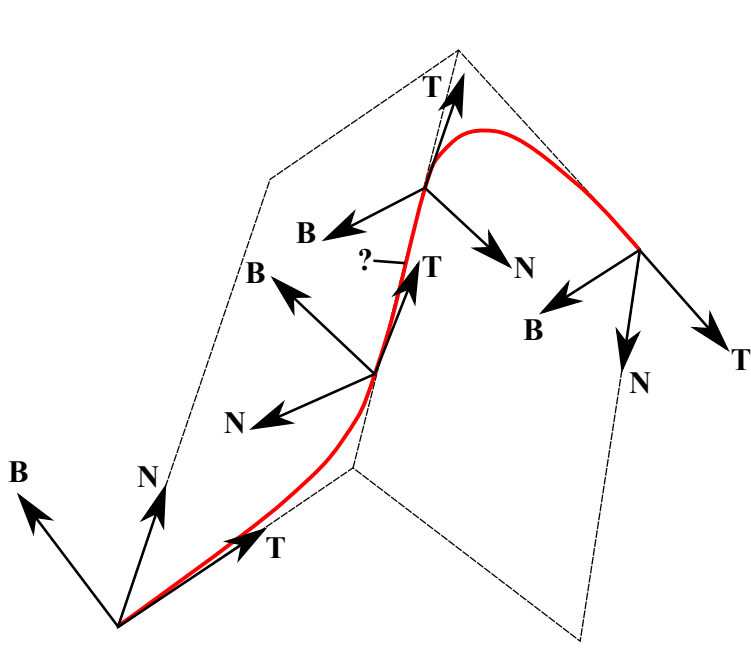

(a) Frenet-Serret Frame

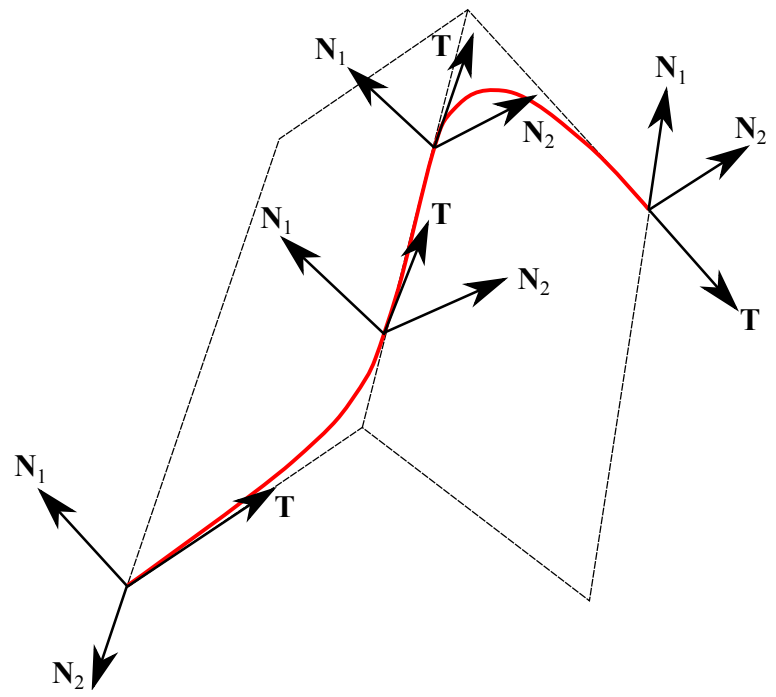

(b) Parallel Transport Frame

Figure 3.7: Comparison between Frenet-Serret and parallel transport frames moving along a curve on a rooftop [68] 


\subsection{Wing Curve Discretization}

In this section, the relevant theories from differential curve geometry are applied to the wing discretization algorithm. First, the wing curve equation is defined using the parallel transport method for curve framing. With the wing curve equation defined, the wing curvature, torsion and total curvature are defined. Finally, the method for which the wing total curvature is used to discretize the wing according to local geometry is shown.

\subsubsection{Wing Curve Framing}

In order for the wing to be discretized accordingly, a parametric space curve and moving coordinate frame that defines the reference wing geometry must be established. In general, a wing can be described but the 1/4-chord line which intersects the camber line at a point that is projected from the 1/4-chord point on each airfoil cross section. This point is shown in Fig. 3.8(a). The spacial location of any $1 / 4$-chord point along the wing is defined by a parametric space curve, $\mathbf{s}_{X}(t)$. An example of this is shown in Fig. 3.8(b).

The moving frame, $\mathcal{F}$, attached to the wing curve must maintain a consistent orientation with respect to the wing, thus the following convention is used:

\section{Moving frame $z$-axis}

For both starboard and port side wings, the coordinate frame $z$ axis is the wing curve tangent, directed outboard.

\section{Moving frame $y$-axis}

The $y$-axis of the coordinate frame is directed toward the trailing edge of the wing for a starboard wing, and is directed toward the leading edge of a port wing.

\section{Moving frame $x$-axis}

Forming the right hand system, the moving frame $x$-axis is always directed upwards. 
Since the frame is of the parallel transport variety, we can specify a parametric spin, $s_{\theta}(t)$, such that the above frame orientation is maintained. Thus, the wing curve used for discretization is a four-dimensional arc length parameterized space curve in which the first three components $\mathbf{s}_{X}(t)$ define the spatial positioning of the $1 / 4$-chord line and the last component $s_{\theta}(t)$ defines the specific coordinate frame spin that maintains a consistent frame orientation as the frame moves from the base to the tip of the wing.

$$
\mathbf{s}(t)=\left\{\begin{array}{l}
\mathbf{s}_{X}(t) \\
s_{\theta}(t)
\end{array}\right\}
$$

The equations used to define the components $\mathbf{s}$ can be any set of parametric equations that posses $C^{2}$ continuity on the interval $0 \leq t \leq 1$. Each component of $\mathbf{s}$ can be a single parametric equation with $C^{2}$ continuity, or a parametric spline with the condition that $C^{2}$ continuity is maintained at the control points. For instance, the wing curve can be described by a cubic b-spline. Cubic b-splines are twice continuously differentiable, thus maintaining $C^{2}$ continuity at the con-

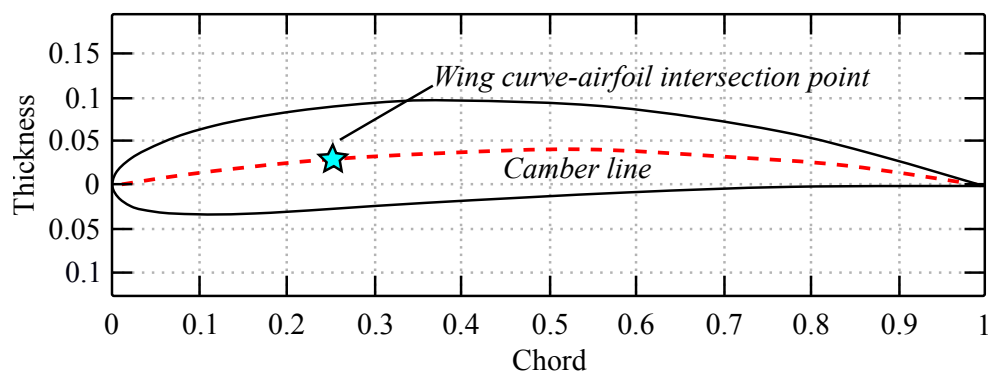

(a) Location of 1/4-chord line airfoil intersection

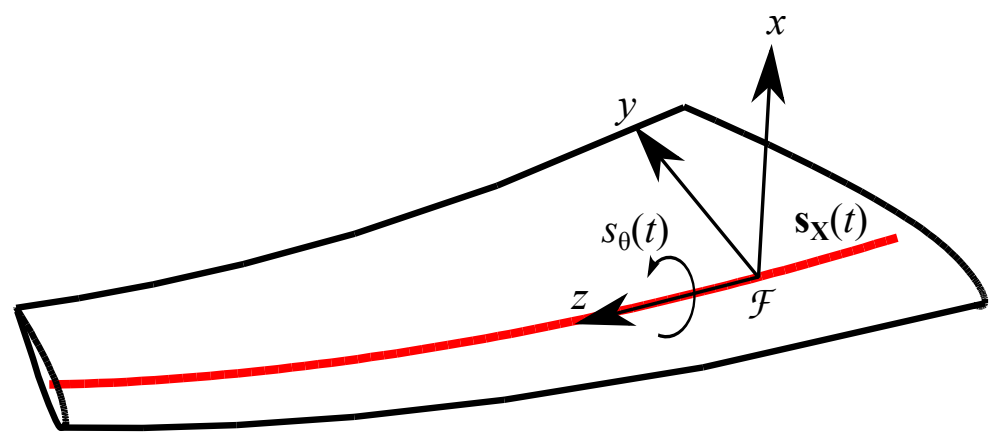

(b) Reference wing with 1/4-chord curve and parallel transport frame

Figure 3.8: Definition of wing curve framing 
trol points. Any airfoil shape can be used insofar as the space curve and parallel transport frame described above are applied consistently along the wing span.

\subsubsection{Differential Wing Curve Geometry}

With the wing curve defined, we can now examine the differential curve geometry properties of the curve. As mentioned, the wing curve is defined using the parallel transport method for curve framing. The coordinate frame is parallely transported if in the limit, there is no difference in orientation between two adjacent coordinate frames. Consider two arbitrary points on the curve, $\mathbf{s}\left(t_{i}\right)$ and $\mathbf{s}\left(t_{i+1}\right)$, which are spanned vectorally by $\mathbf{b}=\mathbf{s}\left(t_{i+1}\right)-\mathbf{s}\left(t_{i}\right)$. Similarly, the orientation of the frame $\mathcal{F}\left(t_{i}\right)$ with respect to $\mathcal{F}\left(t_{i-1}\right)$ is defined by the local pitch, roll and yaw rotations about about the $x_{i^{-}}, y_{i^{-}}$, and $z_{i^{-}}$axes, respectively. Here, since we are concerned with wing geometry, the pitch, roll and yaw rotations correspond to rotations of local sweep, $\Lambda_{i}$, about the $x_{i}$-axis, cant, $\Gamma_{i}$, about the $y_{i}$-axis, and twist, $\alpha_{i}$, about the $z_{i}$-axis, respectively. Thus the total rotation matrix between the two coordinate frame orientations is the multiplication of the individual sweep, cant and twist rotations about their respective axes

$$
\mathbf{R}_{i}=\mathbf{R}_{x}\left(\Lambda_{i}\right) \mathbf{R}_{y}\left(\Gamma_{i}\right) \mathbf{R}_{z}\left(\alpha_{i}\right)
$$

where $\mathbf{R}_{x}\left(\Lambda_{i}\right), \mathbf{R}_{y}\left(\Gamma_{i}\right)$, and $\mathbf{R}_{z}\left(\alpha_{i}\right)$ are the sweep, cant and twist rotations about the local $x_{i^{-}}$, $y_{i^{-}}$, and $z_{i^{-}}$axes, respectively. The individual rotations are given by

$$
\mathbf{R}_{x}=\left[\begin{array}{ccc}
1 & 0 & 0 \\
0 & \cos \Lambda & -\sin \Lambda \\
0 & \sin \Lambda & \cos \Lambda
\end{array}\right], \quad \mathbf{R}_{y}=\left[\begin{array}{ccc}
\cos \Gamma & 0 & \sin \Gamma \\
0 & 1 & 0 \\
-\sin \Gamma & 0 & \cos \Gamma
\end{array}\right], \quad \mathbf{R}_{z}=\left[\begin{array}{ccc}
\cos \alpha & -\sin \alpha & 0 \\
\sin \alpha & \cos \alpha & 0 \\
0 & 0 & 1
\end{array}\right]
$$

Two arbitrary coordinate frame orientations can be associated with any two points along the wing curve as discussed above, but from a module perspective, represents the orientation of two adjacent modules. The successive rotations are shown in Fig. 3.9 where we see how the successive rotations are applied to a module with a finite span. In the limit however, as $\left\|\mathbf{b}_{i}\right\| \rightarrow 0$, we see that $\Lambda_{i}, \Gamma_{i}$, and $\alpha_{i}$ all tend to zero, and thus the two coordinate frames are locally parallel, thus the coordinate 


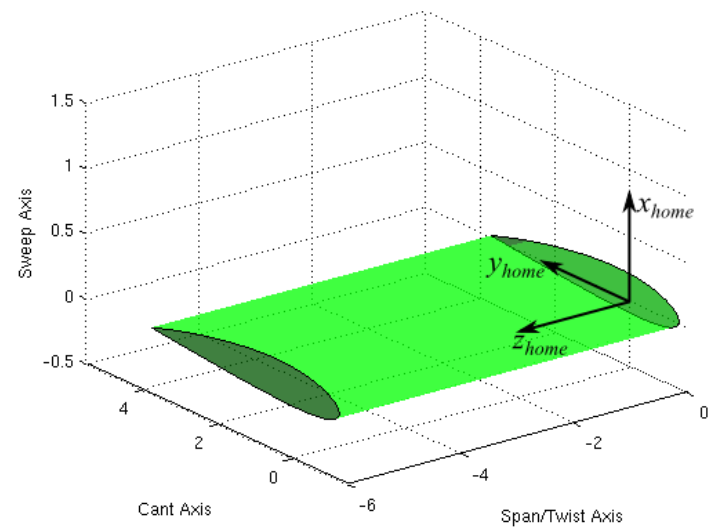

(a) Home position

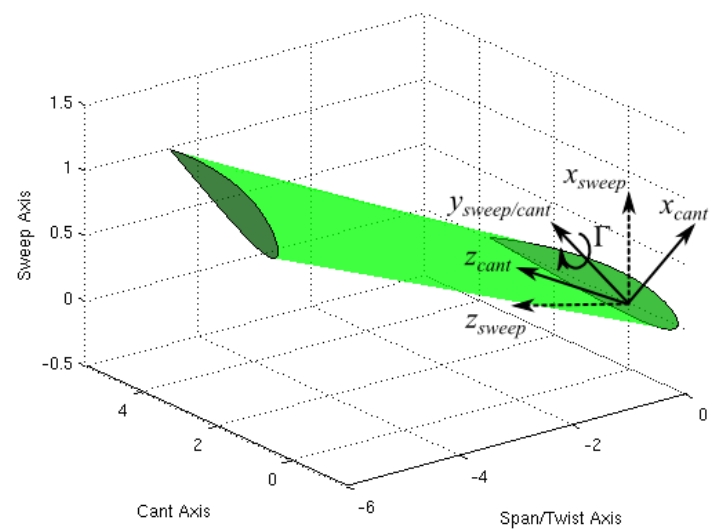

(c) Cant rotation

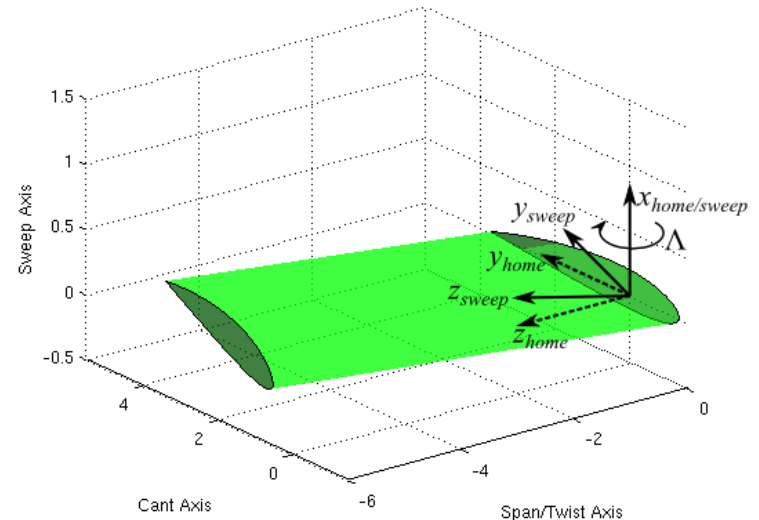

(b) Sweep rotation

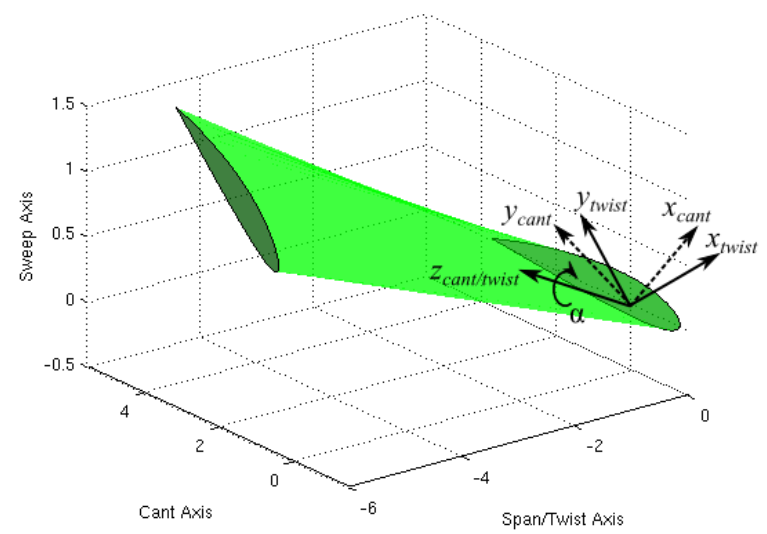

(d) Twist rotation

Figure 3.9: Wing module showing successive sweep, cant and twist rotations

frame is parallely transported along the wing curve.

\subsubsection{Wing Curvature}

The wing curvature, like that of a general curve, is a measure of the tendency for the local tangent axis, the $z$-axis, to deviate from the local orientation. For the parallel transport frame, the curvature defined in Eq. (3.9) is simply the root of the squared sum of the parameterization terms $k_{1}$ and $k_{2}$. However, curvature is an inherent curve property that is not dependent on what type of framing method is used to frame the curve. Thus, $k_{1}$ and $k_{2}$ can effectively be ignored, and the Frenet-Serret 
definition of curvature, Eq. (3.6) can be used. Thus the wing curvature can be defined as

$$
\kappa(t)=\frac{\left|\mathbf{s}_{X}^{\prime \prime}(t) \times \mathbf{s}_{X}^{\prime}(t)\right|}{\left[\mathbf{s}_{X}^{\prime}(t)\right]^{3 / 2}}
$$

As previously mentioned, $\mathbf{s}_{X}(t)$ must maintain $C^{2}$ continuity for all $0 \leq t \leq 1$ due to the second order differential in Eq. (3.14).

\subsubsection{Wing Torsion}

The wing torsion is a measure of the twisting or spin of the moving coordinate frame. As previously described in Section 3.3.3, the parallel transport method of curve framing allows for additional spin to be added which only impacts the curve torsion. For the wing curve, $s_{\theta}(t)$ is defined accordingly so that the frame maintains a consistent orientation as it moves from the wing root to tip. As such, a properly defined $s_{\theta}(t)$ nullifies the parameterization terms $k_{1}$ and $k_{2}$ in Eq. (3.10) and (3.11). This then allows us to define the wing torsion as

$$
\tau(t)=\frac{\left|s_{\theta}^{\prime}(t)\right|}{\left\|\mathbf{s}_{X}^{\prime}(t)\right\|}
$$

Equation (3.15) is essentially a slope of the differential frame spin over the differential wing curve euclidean norm. For unit consistency with respect to the wing curvature, wing curve norm is used in the denominator in lieu of simple the wing curve parameterization differential as in Eq. (3.10). Also, the absolute value of the differential frame spin is used to prevent a possible cancellation of torsion for $s_{\theta}(r)$ that alternates between positive and negative values on the interval $0 \leq t \leq 1$

\subsubsection{Wing Total Curvature}

Total curvature, sometimes referred to as the force of the curve, is essentially a combination of curvature and torsion. At any point along the curve, the total curvature is calculated as

$$
\chi(t)=\sqrt{(\kappa(t))^{2}+(\tau(t))^{2}}
$$

where it is a numerical measure of the instantaneous behaviour of the moving coordinate frame at

any point $t$. A large total curvature indicates any combination of large curvature or torsion, or 
both.

For the reference wing, a complete mathematical description of the wing is given by the wing curve defined in Eq. (3.12). Since $\mathbf{s}$ is required to have $C^{2}$ continuity on the interval $0 \leq t \leq 1$, Eq. (3.16) can be integrated along the entire length of the arc length parameterized wing curve to define the wing total curvature

$$
\zeta=\int_{0}^{1} \chi(t) \mathrm{d} t=\int_{0}^{1} \sqrt{(\kappa(t))^{2}+(\tau(t))^{2}} \mathrm{~d} t
$$

The wing total curvature is thus a measure of the total deviation of the reference wing from a straight, untwisted wing. ${ }^{\dagger}$

\subsubsection{Module Discretization}

Equation (3.17) provides the means by which the wing modules can be discretized according to the local geometric features of the reference wing. As the purpose of the algorithm is to determine the minimum number of modules required to sufficiently emulate a reference wing, the algorithm starts by discretizing the morphing wing into two modules. The algorithm will continue to iterate until an acceptable performance is achieved, thus at any iteration, we can define the average wing total curvature as

$$
\bar{\zeta}=\zeta / m
$$

To determine the nodes at which two adjacent modules are situated, we first note that the wing root and tip are represented by the points $t=0$ and $t=1$, respectively. Since two adjacent modules share a common airfoil rib as a platform of the inboard module and base of the outboard module, we can define the wing root point as $t_{0}=0$, and the wing tip point as $t_{m}=1$ for any $m$-module wing.

\footnotetext{
${ }^{\dagger}$ The wing total curvature is not to be confused with the total curvatured derived from differential curve geometry, although they are similar. The total curvature for the Frenet-Serret Frame and Parallel-Transport Frame are specific to those curve framing methods. For the discretization algorith, the curvature and torsion and extracted from the Frenet-Serret formulae, and Parallel Transport formulation, respectively. As such, the total wing curvature uses the wing curvature (Frenet-Serret) and wing torsion (Parallel-Transport) to describe the instantaneous behavour of the moving coordinate frame.
} 
Since it is required that modules are spaced according to local geometric features, each module should span an equal amount of wing total curvature, or $\bar{\chi}$. Thus using Eq. (3.17), we can determine the nodal spacing vector, $\mathbf{t}$ by starting at sequentially solving

$$
\bar{\zeta}=\int_{t_{i-1}}^{t_{i}} \sqrt{(\kappa(t))^{2}+(\tau(t))^{2}} \mathrm{~d} t, i=1, \cdots, m
$$

In Eq. (3.19) it is plain to see that by starting with the first module, $t_{0}=0$, the only unknown in the equation is $t_{i}$. Equation (3.19) can be solved numerically using any search method such as the bisection or golden section search method. Once Eq. (3.19) is solved for $t_{1}$, then we can continue to solve for all unknown $t_{i}$ values up until $t_{m-1}$ to form the nodal spacing vector

$$
\mathbf{t}=\left\{0, \quad t_{1}, \cdots, t_{i}, \cdots, t_{m-1}, \quad 1\right\}
$$

Equation (3.20) represents the nodal points on the arc-length parameterized wing curve, distributing the wing total curvature equally amongst the number of modules in the current modular wing configuration. As such, a natural module spacing is achieved that will cluster more modules in areas that are geometrically complex, whereas fewer modules are used in areas that are geometrically simple. This allows for the morphing wing to easily achieve geometric similarity between itself and the reference wing for which it must emulate.

\subsubsection{Kinematic Implementation}

In order for the performance of the current wing configuration to be evaluated, the kinematics of the modules must be evaluated so that suitable geometry can be generated for analysis purposes. For instance, the module kinematics along with the airfoil data can be used to generate a point cloud which can be imported to any suitable solid modeling system.

With the set of module nodal points known, the kinematics can be solved for the local sweep, cant, twist, and span of each module. First, it is plain to see that span of each module is simply 
the magnitude of the vector between each sequential node

$$
b_{i}=\left\|\mathbf{s}_{X}\left(t_{i}\right)-\mathbf{s}_{X}\left(t_{i-1}\right)\right\|
$$

To calculate the module sweep and cant, we must look at the difference in orientation between two sequential span vectors. First, we note that $\mathbf{b}_{i}$ acts along the $z_{i}$-axis, thus the local vector form is $\mathbf{b}_{i}^{l}=\left\{\begin{array}{lll}0, & 0, & b_{i}\end{array}\right\}^{\mathrm{T}}$. In the global coordinate frame, $\mathbf{b}_{i}^{g}=\mathbf{s}\left(t_{i}\right)-\mathbf{s}\left(t_{i-1}\right)=\left\{\begin{array}{ll}b_{i, x}, & b_{i, y}, \quad b_{i, z}\end{array}\right\}^{\mathrm{T}}$. Here, $b_{i, x}, b_{i, y}$, and $b_{i, z}$ are the $x, y$, and $z$ components of the module span vector. The local and global representation of $b_{i}$ are related by

$$
\mathbf{b}_{i}^{g}=\left(\prod_{j=1}^{i} \mathbf{R}_{i}\right) \mathbf{b}_{i}^{l}
$$

where $\mathbf{R}_{i}$ is the rotation matrix of module $i$, defined in Eq. (3.13), consisting of the sequential sweep, cant and twist rotations of the module. Since $\mathbf{b}_{i}^{l}$ acts along the $z_{i}$-axis, the twist rotation about the $z_{i}$-axis of module $i$ does not contribute to orientation of $\mathbf{b}_{i}^{l}$. Thus $\mathbf{R}_{z}\left(\alpha_{i}\right)$ can be dropped from Eq. (3.22), which can then be rearranged to form

$$
\left(\prod_{j=i-1}^{1} \mathbf{R}_{j}^{\mathrm{T}}\right) \mathbf{b}_{i}^{g}=\mathbf{R}_{x}\left(\Lambda_{i}\right) \mathbf{R}_{y}\left(\Gamma_{i}\right) \mathbf{b}_{i}^{l}
$$

To simplify the notation, $\mathbf{b}_{i}^{\prime}=\left(\prod_{j=i-1}^{1} \mathbf{R}_{j}^{\mathrm{T}}\right) \mathbf{b}_{i}^{g}=\left\{b_{i, x}^{\prime}, \quad b_{i, y}^{\prime}, \quad b_{i, z}^{\prime}\right\}^{\mathrm{T}}$. After some rearranging, the module sweep and cant can be determined by

$$
\begin{aligned}
& \Lambda_{i}=\arctan 2\left(-b_{i, y}^{\prime}, b_{i, z}^{\prime}\right) \\
& \Gamma_{i}=\arcsin \left(\frac{b_{i, x}^{\prime}}{b_{i}}\right)
\end{aligned}
$$

Finally, the module twist can be calculated. Since the tangent of the wing curve and the direction $\left(z_{i}\right.$-axis) of the module $i$ are not necessarily co-linear, the module twist angle cannot be determine by simply computing the twist at $s_{\theta}\left(t_{i}\right)$. This is due to the discrete nature of the modular morphing wing. Referring to Fig. 3.10, the module twist angle is actually the angle between the $y z$-plane at $\mathbf{s}_{X}\left(t_{i}\right)$ and the module $y$-axis projected on the $y z$-plane at $\mathbf{s}_{X}\left(t_{i}\right)$. The module twist 


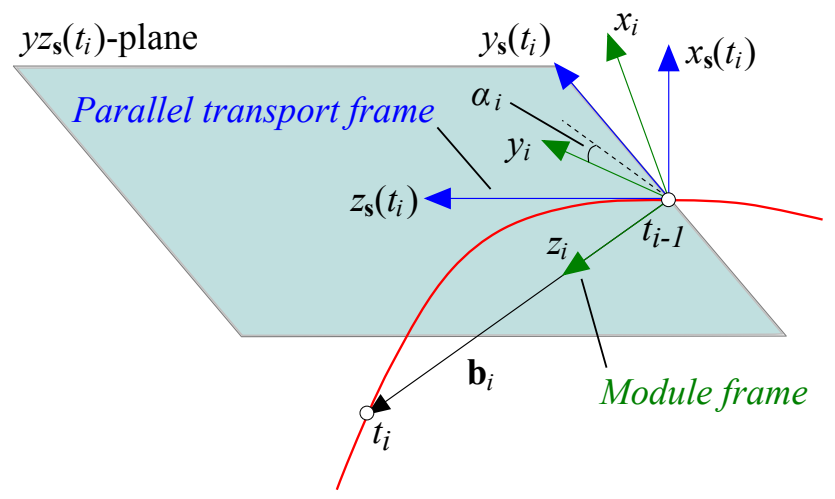

Figure 3.10: Definition of module twist

can now be determined by calculating the angle between the wing curve $z$-axis at $\mathbf{s}_{X}\left(t_{i}\right)$ (normal of the $y z$-plane at $\left.\mathbf{s}_{X}\left(t_{i}\right)\right)$ and the $y$-axis of the module after sweep and dihedral rotations have been computed

$$
\alpha_{k}=\pi / 2-\arccos \left(\mathbf{x}_{\alpha} \cdot \mathbf{x}_{\mathbf{R}}\right)
$$

where

$$
\begin{aligned}
& \mathbf{x}_{\alpha}=\left\{-\sin \left(s_{\theta}\left(t_{i}\right)\right), \quad \cos \left(s_{\theta}\left(t_{i}\right)\right), \quad 0\right\}^{\mathrm{T}} \\
& \mathbf{x}_{\mathbf{R}}=\left(\prod_{j=1}^{i-1} \mathbf{R}_{j}\right) \mathbf{R}_{x}\left(\Lambda_{i}\right) \mathbf{R}_{y}\left(\Gamma_{i}\right)\left\{\begin{array}{lll}
0, & 1, & 0
\end{array}\right\}^{\mathrm{T}}
\end{aligned}
$$

Eq. (3.21), (3.24), (3.25), and (3.26) are joint variables for each wing module. The position of each wing module in the global coordinate frame can be calculated recursively using Eq. (3.23), whereas the orientation of each module in the global coordinate frame is

$$
\mathbf{R}_{i}^{g}=\prod_{j=1}^{i} \mathbf{R}_{j}
$$

We note that in the limit as $m \rightarrow \infty, b_{i}, \Lambda_{i}, \Gamma_{i}$ and $\alpha_{i}$ all tend to zero. Also in the limit, the orientations of module $i$ and the wing curve moving coordinate frames align. 


\subsection{Case Study}

In order to show the effectiveness of the discretization algorithm, a hypothetical reference wing is used as a case study. First, the reference wing for case study is defined. This is followed by a summary of the geometry discretization and performance analysis steps of the algorithm. The final wing configuration is given, followed by a short discussion on results of the analysis.

\subsubsection{Reference Wing Curve Definition}

For the case study, an arbitrary reference wing curve is defined by using the set of points listed in Table 3.1 to form a spline for each component. Each b-spline forms one of the components of Eq. (3.12) is valid on the interval $0 \leq t \leq 1$. The wing and coordinate frame setup is shown in Fig. 3.11(a). Also shown is a graph with the wing curve splines plotted on the same graph. The wing itself has a taper ratio of 0.1 . It has three main sections; the inner section with a moderate sweep and small cant; the middle section with a slightly larger sweep and cant, and; an outer portion with a winglet. A NACA 0015 airfoil was used for the wing. This wing is entirely hypothetical, however it does resemble a swept wing with a blended winglet, and where the span is constrained to a specific value. From the module performance analysis, the $C_{L} / C_{D}$ performance target of the wing was shown to be approximately 26.5, i.e. the maximum performance attained from the discretized wings. Other unconventional wing configurations have been shown to be desirable for certain flight regimes and requirements [37].

Table 3.1: Points used to form wing curve b-spline

\begin{tabular}{lllllllllllll}
\hline \multicolumn{1}{c}{ Wing curve spline coordinates } \\
$\mathbf{t}$ & $\mathbf{0}$ & $\mathbf{0 . 1}$ & $\mathbf{0 . 2}$ & $\mathbf{0 . 3}$ & $\mathbf{0 . 4}$ & $\mathbf{0 . 5}$ & $\mathbf{0 . 6}$ & $\mathbf{0 . 7}$ & $\mathbf{0 . 8}$ & $\mathbf{0 . 9}$ & $\mathbf{1}$ \\
\hline$s_{x}$ & 0.00 & 3.00 & 6.72 & 11.43 & 17.58 & 25.97 & 38.01 & 57.14 & 91.43 & 171.43 & 571.43 \\
& & & & & & & & & & & \\
$s_{y}$ & 0.00 & 36.85 & 80.40 & 130.65 & 187.60 & 251.25 & 321.60 & 398.65 & 482.40 & 572.85 & 670.00 \\
$s_{z}$ & 0 & 250 & 500 & 750 & 1000 & 1250 & 1500 & 1750 & 2000 & 2250 & 2500 \\
$s_{\theta}$ & 0.0227 & 0.0250 & 0.0278 & 0.0313 & 0.0357 & 0.0417 & 0.050 & 0.0625 & 0.0833 & 0.125 & 0.250 \\
\hline
\end{tabular}




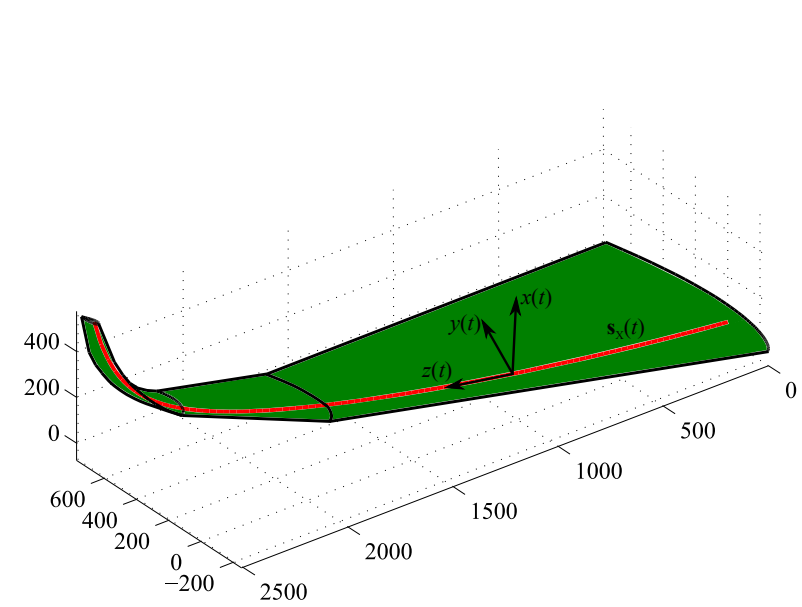

(a) Reference wing isometric view

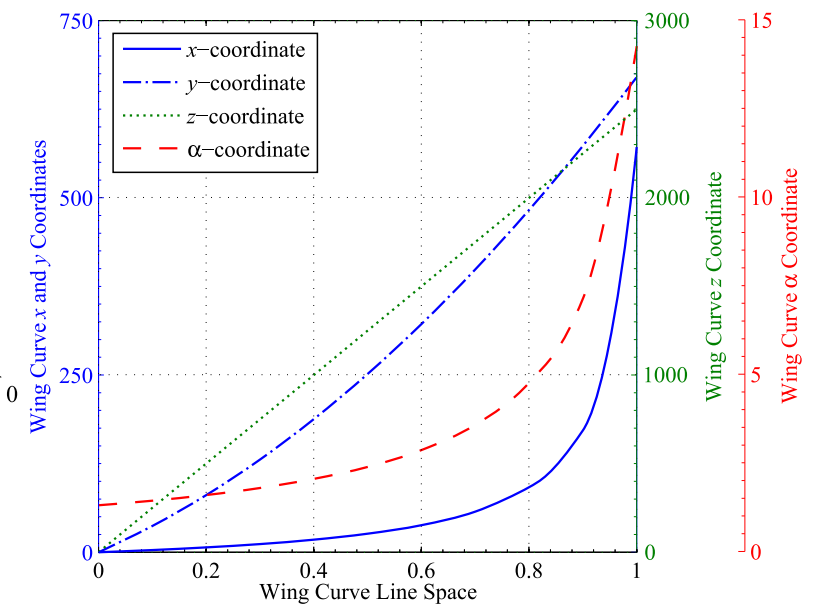

(b) Reference wing coordinates

Figure 3.11: Definition of reference wing used for discretization algorithm case study

\subsubsection{Morphing Wing Geometry Discretization}

With the wing curve defined using a b-spline with the coordinates listed in Table 3.1, the morphing wing can be discretized using the total curvature of the reference wing. This represents the first step of the discretization algorithm, and the geometry discretization would be performed as additional modules are added to the morphing wing system until a suitable performance is achieved (shown in the following section). The geometry discretization step of the algorithm is detailed here using the reference wing as an example.

Due to the reference wing geometry, it is expected that when discretized, modules will be clustered in the region where the winglet curves upward from the remaining portion of the wing. This is illustrated in Fig. 3.12 where the reference wing total curvature is plotted. The solid line represents the total curvature, $\chi(t)(\mathrm{Eq}(3.16))$; the vector sum of the wing curvature and wing torsion at any point $t$ along the curve. If integrated from $t=0$ to $t=1$, the wing total curvature is calculated as $\zeta=4.1825 \times 10^{-4}$. Also shown along the curve are the components of the nodal spacing vector for a selection of discretized wings. The horizontal spacing of the markers reflects the components of the nodal spacing vector $\mathbf{t}$ for the various discretized wing. As seen, there is a peak in local contribution of total curvature at $t \approx 0.94$, and then drops off as $t \rightarrow 1$. Because 
of the sudden rise in curvature, corresponding to the location where the winglet begins, there are more modules located in this wing region, representative by the horizontal clustering of the markers within this region.

Table 3.2: Nodal point distribution and module kinematics of the a selection of discretized wings

\begin{tabular}{|c|c|c|c|c|c|c|c|}
\hline $\mathbf{m}$ & $\bar{\zeta} \times 10^{-4}$ & $\mathbf{i}$ & $t_{i}$ & $\Lambda[\mathrm{deg}]$ & $\Gamma[\mathrm{deg}]$ & $\alpha[\mathrm{deg}]$ & b [units] \\
\hline \multirow[t]{2}{*}{2} & 2.90125 & 1 & 0.89 & -14.2 & 4.0 & 1.3 & 2312.8 \\
\hline & & 2 & 1 & -4.7 & 51.3 & 6.8 & 496.0 \\
\hline \multirow[t]{3}{*}{3} & 1.39417 & 1 & 0.83 & -13.8 & 2.9 & 1.3 & 2151.5 \\
\hline & & 2 & 0.93 & -5.9 & 20.5 & 3.9 & 272.7 \\
\hline & & 3 & 1 & 3.8 & 38.0 & 6.3 & 401.7 \\
\hline \multirow[t]{4}{*}{4} & 1.04563 & 1 & 0.77 & -13.4 & 2.3 & 1.3 & 1989.7 \\
\hline & & 2 & 0.83 & -5.9 & 12.6 & 3.0 & 330.7 \\
\hline & & 3 & 0.94 & 0.6 & 19.2 & 2.2 & 156.8 \\
\hline & & 4 & 1 & 3.9 & 30.2 & 4.4 & 355.0 \\
\hline \multirow[t]{5}{*}{5} & 0.83650 & 1 & 0.71 & -12.9 & 1.9 & 1.3 & 1812.1 \\
\hline & & 2 & 0.86 & -5.9 & 8.4 & 2.2 & 423.6 \\
\hline & & 3 & 0.92 & -0.4 & 14.3 & 2.1 & 152.9 \\
\hline & & 4 & 0.95 & 1.3 & 15.9 & 1.8 & 123.0 \\
\hline & & 5 & 1 & 3.8 & 25.6 & 4.1 & 323.9 \\
\hline \multirow[t]{6}{*}{6} & 0.69708 & 1 & 0.63 & -12.3 & 1.5 & 1.3 & 1620.8 \\
\hline & & 2 & 0.83 & -5.8 & 5.8 & 1.7 & 535.0 \\
\hline & & 3 & 0.89 & -1.1 & 11.4 & 2.0 & 167.2 \\
\hline & & 4 & 0.93 & 0.6 & 12.1 & 1.4 & 107.9 \\
\hline & & 5 & 0.96 & 1.5 & 14.0 & 1.8 & 107.2 \\
\hline & & 6 & 1 & 3.7 & 22.3 & 4.2 & 300.6 \\
\hline \multirow[t]{7}{*}{7} & 0.59750 & 1 & 0.56 & -11.8 & 1.3 & 1.3 & 1434.5 \\
\hline & & 2 & 0.80 & -5.7 & 4.3 & 1.3 & 641.1 \\
\hline & & 3 & 0.87 & -1.7 & 9.3 & 1.9 & 189.5 \\
\hline & & 4 & 0.91 & 0.2 & 9.9 & 1.3 & 107.3 \\
\hline & & 5 & 0.94 & 0.9 & 10.8 & 1.4 & 86.6 \\
\hline & & 6 & 0.96 & 1.6 & 12.6 & 1.9 & 98.1 \\
\hline & & 7 & 1 & 3.6 & 19.9 & 4.3 & 281.9 \\
\hline
\end{tabular}




\begin{tabular}{|c|c|c|c|c|c|c|c|}
\hline $\mathbf{m}$ & $\bar{\zeta} \times 10^{-4}$ & $\mathbf{i}$ & $t_{i}$ & $\Lambda$ [deg] & $\Gamma[\mathrm{deg}]$ & $\alpha[\mathrm{deg}]$ & $\mathbf{b}$ [units] \\
\hline \multirow[t]{8}{*}{8} & 0.52281 & 1 & 0.50 & -11.3 & 1.2 & 1.3 & 1271.1 \\
\hline & & 2 & 0.77 & -5.5 & 3.3 & 1.0 & 721.5 \\
\hline & & 3 & 0.85 & -2.1 & 7.6 & 1.8 & 217.9 \\
\hline & & 4 & 0.89 & -0.1 & 8.5 & 1.2 & 113.5 \\
\hline & & 5 & 0.92 & 0.5 & 8.9 & 1.1 & 82.1 \\
\hline & & 6 & 0.94 & 1.0 & 9.8 & 1.4 & 75.3 \\
\hline & & 7 & 0.96 & 1.6 & 11.6 & 1.9 & 92.1 \\
\hline & & 8 & 1 & 3.5 & 18.0 & 4.4 & 266.4 \\
\hline \multirow[t]{9}{*}{9} & 0.46472 & 1 & 0.45 & -11.0 & 1.1 & 1.3 & 1135.0 \\
\hline & & 2 & 0.74 & -5.3 & 2.6 & 0.8 & 770.6 \\
\hline & & 3 & 0.83 & -2.4 & 6.2 & 1.6 & 251.5 \\
\hline & & 4 & 0.88 & -0.4 & 7.4 & 1.2 & 122.9 \\
\hline & & 5 & 0.91 & 0.3 & 7.7 & 1.0 & 82.9 \\
\hline & & 6 & 0.93 & 0.7 & 8.2 & 1.1 & 68.5 \\
\hline & & 7 & 0.95 & 1.1 & 9.0 & 1.4 & 68.2 \\
\hline & & 8 & 0.97 & 1.7 & 10.7 & 2.0 & 87.9 \\
\hline & & 9 & 1 & 3.4 & 16.5 & 4.5 & 253.1 \\
\hline \multirow[t]{10}{*}{10} & 0.41825 & 1 & 0.40 & -10.6 & 1.0 & 1.3 & 1022.8 \\
\hline & & 2 & 0.71 & -5.1 & 2.1 & 0.7 & 791.3 \\
\hline & & 3 & 0.81 & -2.7 & 5.1 & 1.4 & 289.5 \\
\hline & & 4 & 0.86 & -0.6 & 6.5 & 1.1 & 134.7 \\
\hline & & 5 & 0.89 & 0.1 & 6.8 & 0.9 & 86.3 \\
\hline & & 6 & 0.92 & 0.4 & 7.1 & 0.9 & 66.8 \\
\hline & & 7 & 0.93 & 0.7 & 7.6 & 1.1 & 60.1 \\
\hline & & 8 & 0.95 & 1.0 & 8.4 & 1.4 & 63.3 \\
\hline & & 9 & 0.97 & 1.7 & 10.0 & 2.1 & 84.8 \\
\hline & & 10 & 1 & 3.4 & 15.2 & 4.6 & 241.5 \\
\hline
\end{tabular}

A summary of the distribution of nodal points and the kinematics of the various modules is shown in Table 3.2. Here, values for the first nine iterations of the geometry discretization are shown. For the nodal points, $t_{0}=0$ is omitted as it represents the beginning of each wing. However, $t_{m}=1$ is shown along with the remaining interior nodal points, which for each module, indicates the point along the wing curve where the modules end. It is worth noting that as the number of modules increase, the average kinematic values per module begin to decrease, eventually tending towards zero, thus forming a continuous wing. This is reflected in Fig. 3.13 where the average module kinematic values all tend to zero as modules are increased. 


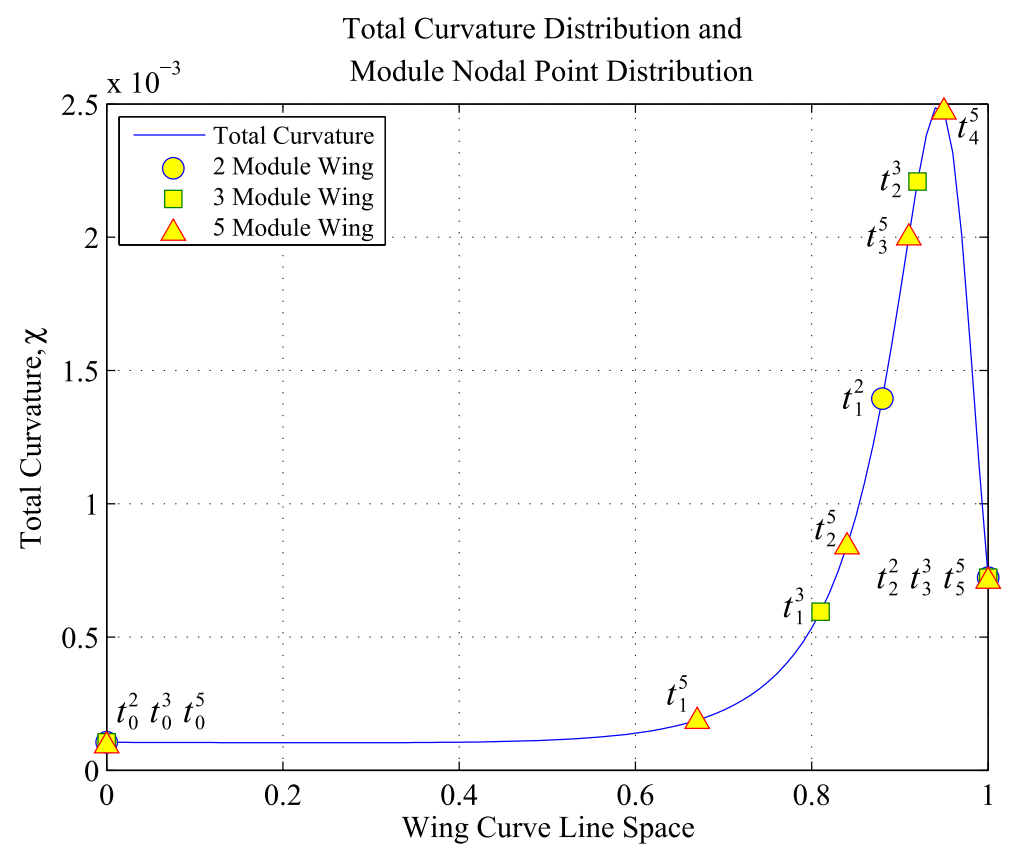

Figure 3.12: Total curvature distribution of the reference wing curve showing nodal point distribution for selected wing configurations

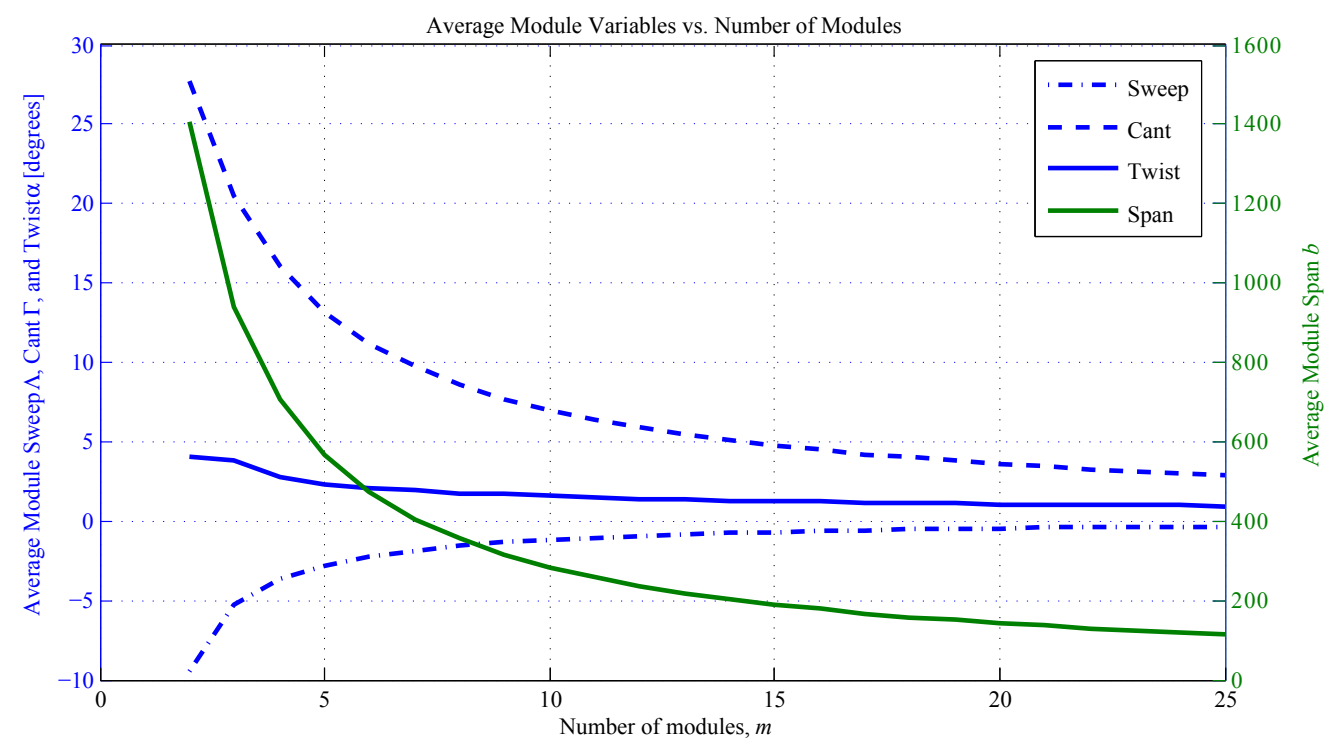

Figure 3.13: Wing module average variable values

\subsubsection{Morphing Wing Performance Evaluation}

As mentioned, the module kinematics are then used to form the geometry of the discretized wing by sequentially calculating the position and orientation, or pose, of each wing module in relation to the previous module. The module kinematics allow for the geometric construction of the wing for the 
implementation purposes. The geometric wing information can be fed into a suitable CAD program to generate a solid model, or can be input directly into a mesh generation program with some type of CAD editing capabilities. For this study, ANSYS@ Gambit ${ }^{\mathrm{TM}}$ is used for mesh generation, and ANSYSC Fluent ${ }^{\mathrm{TM}}$ is used to solve the aerodynamics. Each wing is meshed with structured and unstructured meshes within the control volume, with a total of approximately 12 million cells. The number of cells along the span of each module is scaled according to the module length to provide constant node spacing along the entire span of the wing with $y^{+} \approx 1$ (see Appendix A for more details). Each configuration is set to $A o A=5^{\circ}$, and flown at $M_{\infty}=0.3$ at standard sea level and a mean chord Reynolds number $R e \approx 3.5 \times 10^{6}$. A steady, implicit, density-based, second-order upwind solver is used with the Spalart-Allmaras viscous model. The lift-to-drag ratio is used here as a performance index due to its importance in regards to cruise performance, however other indices can be used for different flight requirements.

Three discretized wings are shown in Fig. 3.14, and a summary of discretized wing performances is shown in Fig. 3.15. For the first loop of the discretization algorithm, the wing is divided into two modules as shown in Fig. 3.14(a). When comparing the two-module discretized wing to the reference wing (Fig. 3.11(a)), we see that the wing tip region is not sufficiently emulated, solely due to the limited number of modules available to the morphing wing. As a result, the corresponding $C_{L} / C_{D}$ ratio is approximately 21.5. With additional modules, the modular morphing wing is better suited to emulate the winglet region of the reference wing. This is reflected not only in the physical wing configurations, but also in the flight performance data.

As modules are increased, we see that $C_{L}$ steadily increases, $C_{D}$ decreases to a minimum of approximately 0.0169 , and then steadily increases, and $C_{L} / C_{D}$ increases asymptotically to a maximum of approximately 26.4. The configurations corresponding to the minimum $C_{D}$ and the maximum $C_{L} / C_{D}$ are the four-module wing (Fig. 3.14(b)) and the seven-module wing respectively. However, we notice that there are no appreciable gains and $C_{L} / C_{D}$ begins to decline after seven modules. In fact, $C_{L} /\left.C_{D}\right|_{m=6}=26.4002$ and $C_{L} /\left.C_{D}\right|_{m=7}=C_{L} /\left.C_{D}\right|_{\max }=26.4174$. Since $C_{L} /\left.C_{D}\right|_{m=6} \approx C_{L} /\left.C_{D}\right|_{m=7}$, the six-module wing (Fig. 3.14(c)) is the configuration with the mini- 


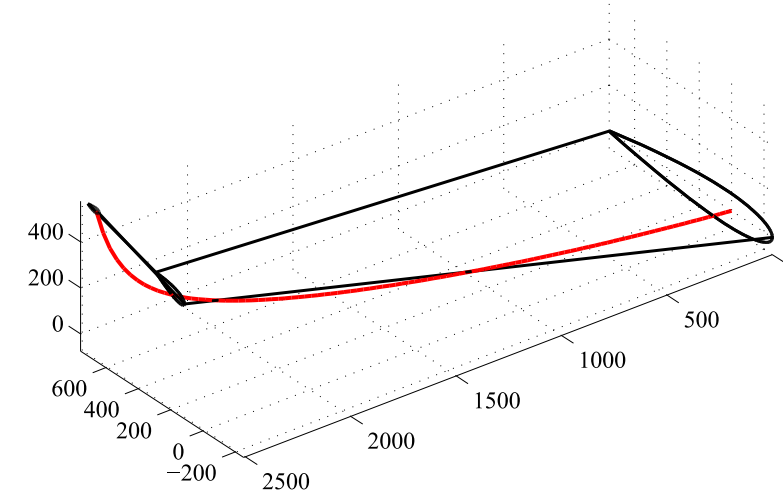

(a) 2 module wing

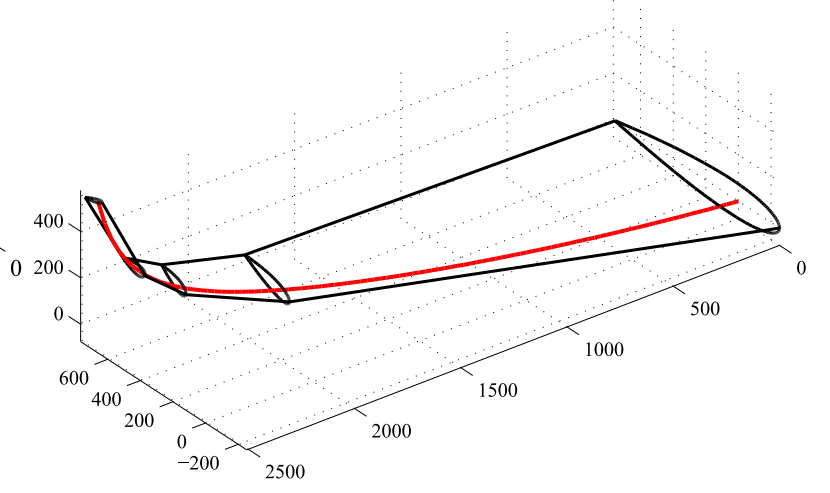

(b) 4 module wing

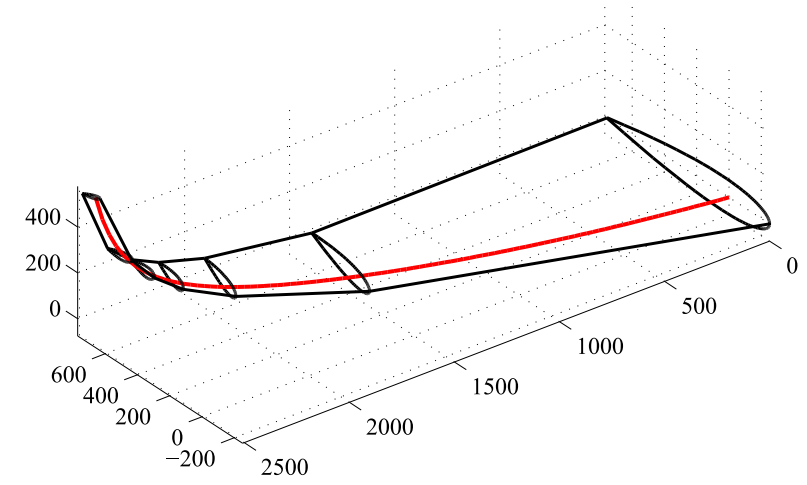

(c) 6 module wing

Figure 3.14: Wing configurations for case study discretization algorithm example

mum number of modules that maximizes the flight performance. Comparing this value to that of the reference performance, we see that they are essentially identical, thus we can confidently state that the six-module wing sufficiently emulates the reference wing shape. In fact, the six-module configuration meets the stopping criteria listed in Section 3.2.3.

On the other hand, if drag reduction was the sole performance requirement, then we see that the final wing configuration would a four-module morphing wing. We note that $\left.C_{D}\right|_{m=4}=0.0170$ and $\left.C_{D}\right|_{m=4}=0.0169$, and $C_{L} /\left.C_{D}\right|_{m=4}=25.0807$ which is within $5.06 \%$ of $C_{L} /\left.C_{D}\right|_{m=7}$. Thus the four-module wing module suffers only a minimum decrease in $C_{L} / C_{D}$, while having a significantly lower $C_{D}$. In fact, four-, five-, and six-module wings provide the aircraft with a balance between low drag and a high lift to drag ratio.

Choosing the six-module wing to be the final configuration for the morphing wing, Fig. 3.16(a) 
shows the spacing of the modules along the wing in its straight, untwisted state. The spacing of the module was determined by the discretization algorithm. The remaining images in Fig. 3.16 shows the stages of the morphing maneuver at $25 \%, 50 \%, 75 \%$ and $100 \%$.

A full aerodynamic analysis of the wing is beyond the scope of this dissertation, but some observations on the performance results are given. From the CFD analysis, there are adverse flow conditions in regions near the module boundaries due to the discontinuity between two adjacent module surfaces and results in increased drag. Any cant-variation between two adjacent modules results in mixing of the two boundary layers from each module. This phenomenon is compounded as additional modules are added to the system, however the effect of each contribution is diminshed, corresponding to a decrease in local module cant angles. The drop in $C_{D}$ is attributed to the two and three module wings failing to accurate describe the geometry of the reference wing. Also, as fewer modules are used in these configurations, the module cant values are higher, thus contributing to the mixing of adjacent boundary layers. The pressure coefficient distribution for the two, four and six-module wing configurations is shown in Fig. 3.17. As additional modules are added, there is

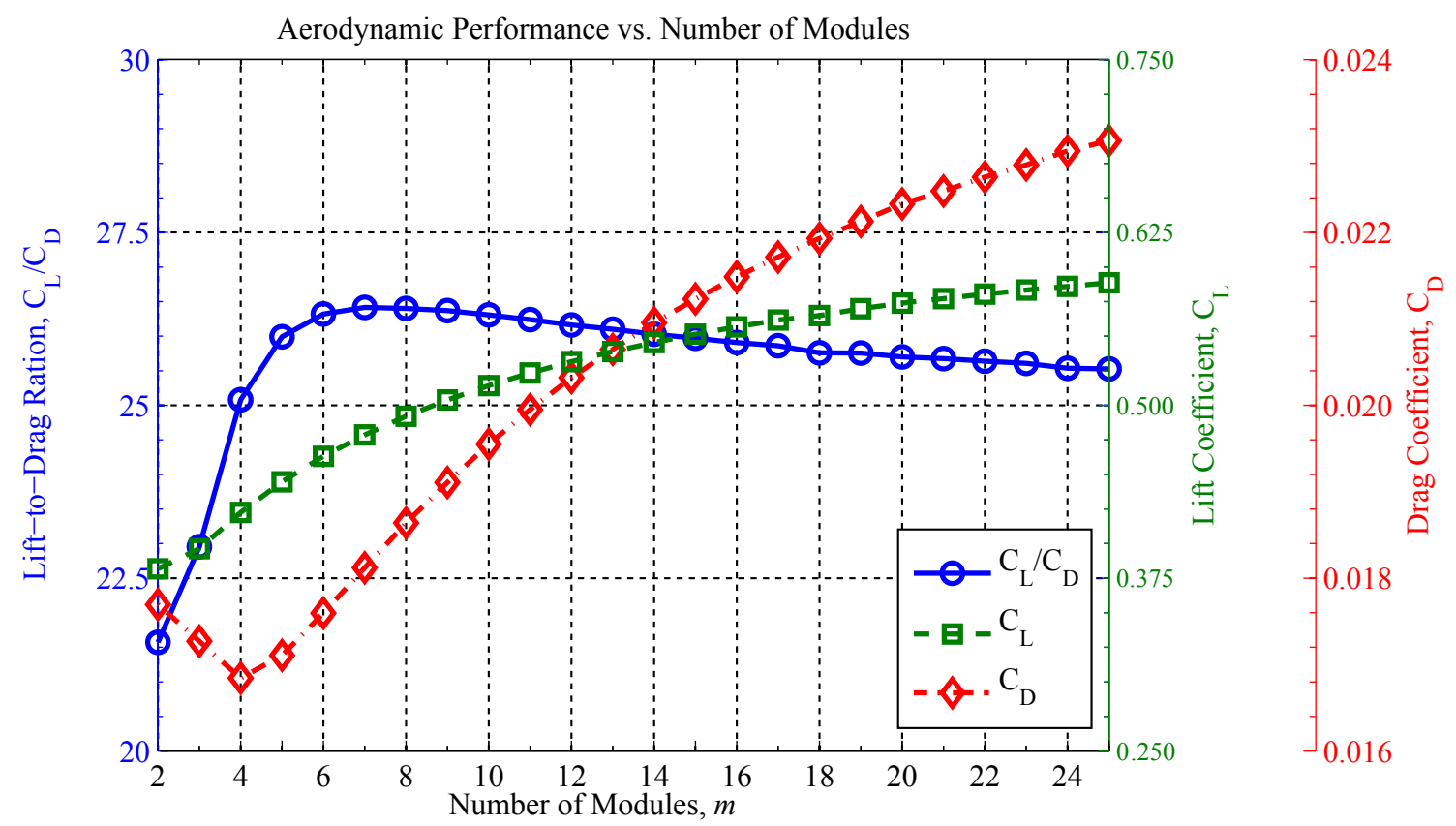

Figure 3.15: Case study performance evaluation results showing lift, drag and lift-to-drag ratio distribution 


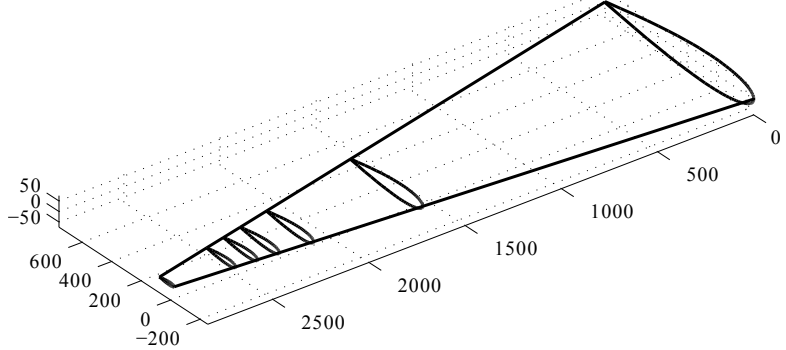

(a) $0 \%$ morphed

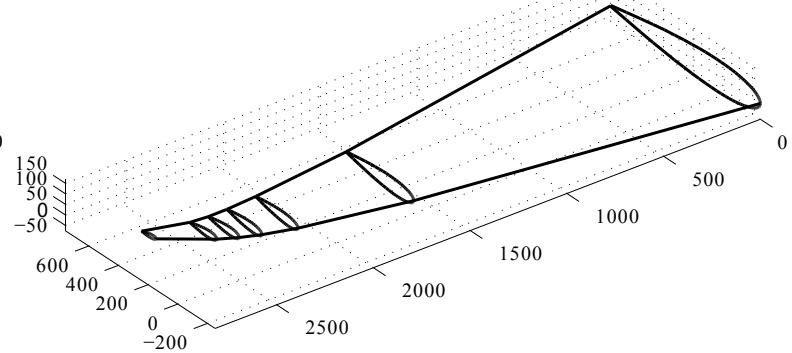

(b) $25 \%$ morphed

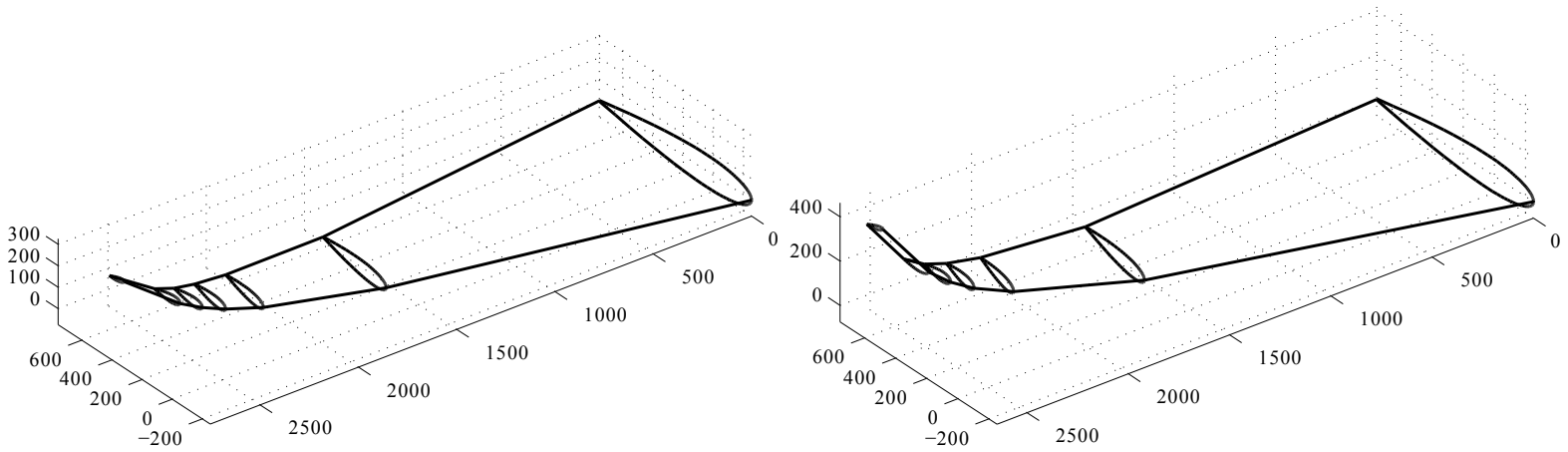

(c) $50 \%$ morphed

(d) $75 \%$ morphed

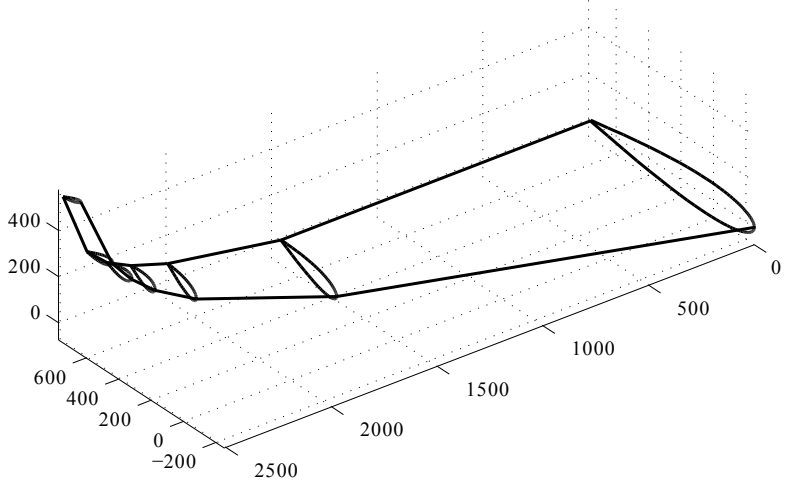

(e) $100 \%$ morphed

Figure 3.16: Example morphing maneuver showing a transition from a straight, untwisted wing, to a fully morphed state

an increase in wetted wing surface area which also leads to an increase skin friction drag. This buildup of drag begins at some point to outweigh the benefits in performance associated with adding modules. This phenomenon is seen with the decrease in $C_{L} / C_{D}$ beyond the seven-module wing configuration for the case study, and could also be associated with the fact that a hypothetical reference wing was used in the case study. These effects will differ depending on the reference wing used. However, we notice that as more modules are added, the discontinuity between two 


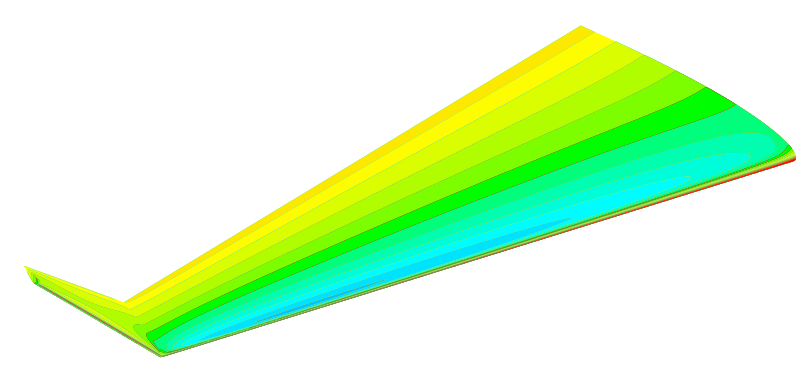

(a) 2 module wing

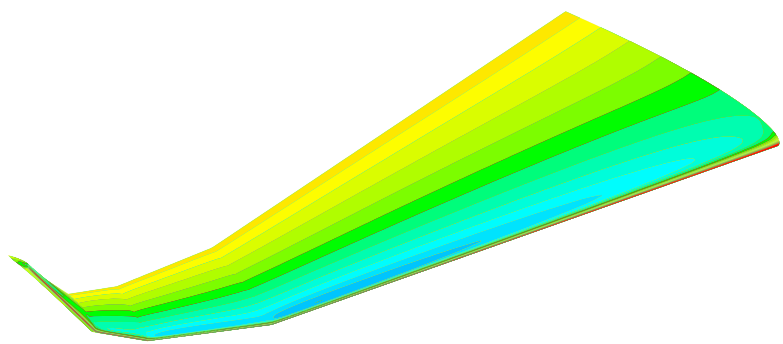

(b) 4 module wing

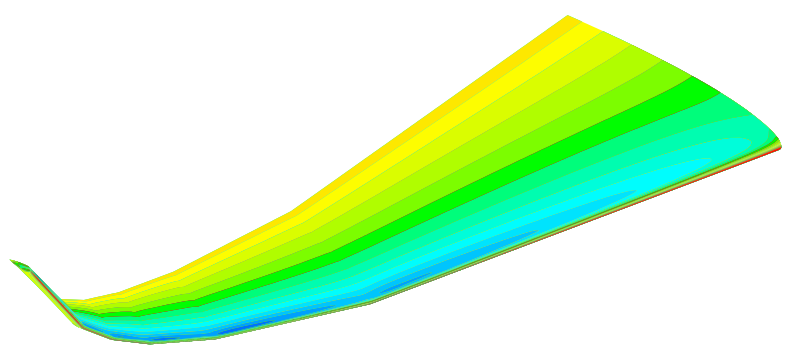

(c) 6 module wing

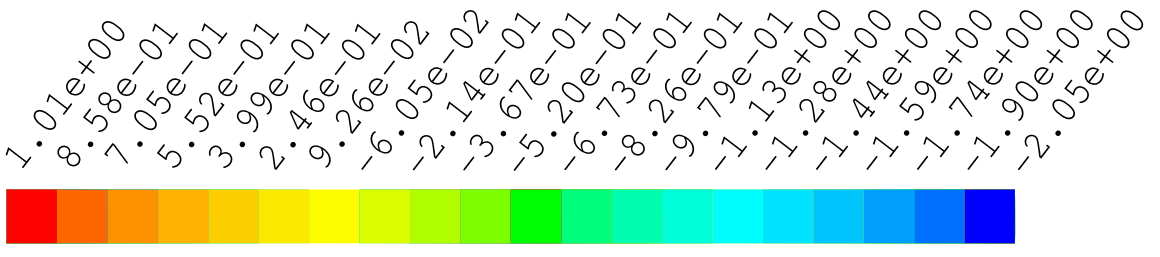

Figure 3.17: Pressure coefficient distribution for three discretized wing configurations

adjacent module surfaces begins to decrease, and the lift, drag and lift-to-drag ratio all begin to level off. There is thus a trade-off between performance gains, and number of modules with respect to the increase in drag associated with both the discontinuity between modules, and total wetted surface area. It is near the module boundary regions that further analysis into the flow is required, particularly how the flow is affected for different module kinematic values.

\subsection{Summary}

In this chapter, a discretization method for the development of a modular morphing wing was presented. The new and unique algorithm is meant to provide the minimum number of morphing wing modules required to satisfy a particular flight requirement. By using a reference wing with desirable performance qualities, the discretization uses the reference wing geometry to discretize the morphing wing into a set number of modules. Differential curve geometry was shown to be 
an effective means to not only mathematically describe the reference wing, but was also shown to determine a natural module spacing that clusters more modules in areas of large wing total curvature, and spacing out fewer modules in areas of small wing total curvature. The natural spacing of the modules allows for the modular morphing wing to easily emulate the geometry of the reference wing, and a case study was performed to show how the performance of the modular wing changes with the addition of modules. 


\section{Chapter 4}

\section{Wing Module Kinematic Architecture}

In this chapter, the kinematic architectural requirements of the wing module are presented. First, the high-level mobility, connectivity and constraint requirements of the wing truss module are introduced. To satisfy the constraint requirements of the poly-morphing wing mechanism, two distinct methodologies are presented. For each constraint methodology, a detailed mobility analysis is performed, along with the enumeration of module configurations for different morphing criteria. A comparison is then made between the two module constraint methodology, and one is selected for further development. Finally, a summary of the chapter is given.

\subsection{Parallel Mechanism Branch Mobility, Connectivity and De- sign Methodologies}

Truss mechanisms, introduced in Section 2.4, are best suited to satisfy the kineto-structural morphing requirements of a morphing wing system. Additionally, truss-based mechanisms are ideal to be used as a development platform for the systematic development of a modular morphing wing due to the features previously outlined. Each module within the truss system is a self contained parallel style mechanism. The base of one module acts a platform for the next, and this setup is repeated as modules are positioned span-wise along the wing until a full wing system is assembled. Each module, in effect, is an isolated mechanical system, however, the modularity of the system allows a single module to be reconfigured according to the morphing requirements at the particular 
span-wise location, thus only a single module need be designed and analyzed. In this section, the mobility, connectivity and design methodology of parallel mechanism branches are discussed.

\subsubsection{Parallel Mechanism Mobility and Connectivity}

In order to understand the kinematic capabilities of a parallel style mechanism, including the wing module, the mobility of the system must be established. The mobility $M$, of a mechanical system is defined using the Chebychev-Gr ubler-Kutzbach criterion (also known as the mobility equation)

$$
M=\lambda\left(n_{l}-n_{j}-1\right)+\sum_{j=1}^{n_{j}} f_{j}
$$

where $\lambda$ is the system order, respectively, $n_{l}$ is the number of links, $n_{j}$ is the number of joints,

and $f_{j}$ is the degree-of-freedom number for the $j^{\text {th }}$ joint. In Eq. (4.1), the system order is defined depending on the motion profile of the system, where $\lambda=3$ for planar motion and $\lambda=6$ for spatial motion. In other words, the system order equals the total degrees-of-freedom possible for the mechanism.

Connectivity is the total degrees-of-freedom within any branch of the parallel robot and is the sum of the individual joint degrees-of-freedom for the particular branch. In order for a parallel mechanism to maintain its kinematic integrity, limits on branch connectivity must be maintained. Tsai [69] has shown using Euler's equation that for each branch, the following relationship between the mobility, connectivity and system order must hold

$$
M \leq C_{k} \leq \lambda
$$

where $C_{k}$ is the connectivity of the $k^{t h}$ branch. In Eq. (4.2), we see that the connectivity of any branch must be no less than the mobility of the robot, and no greater than the system order, thus establishing the robot mobility and branch connectivity relationship requirement. The physical interpretation of Eq. (4.2) is such that branches with a connectivity less than the mobility of the system introduce undesirable constraints on the moving platform, whereas branches with a connectivity greater than the system order posses redundant degrees-of-freedom and may be loose. 
For the former, the introduction of constraints as such is undesirable, as systems are designed to achieve a specific mobility. By introducing constraints in this manner, the mobility of the system is reduced to below what is required, and thus cannot be actuated according to the design requirements. In the latter case, a loose branch within the parallel robot structure on the other hand may introduce additional degrees-of-freedom for the platform which may not be controllable, thus altering the kinematic behaviour of the system in a negative manner. This scenario might also results in a parallel mechanical system that cannot in general be actuated according to the specific design requirements. Therefore the branches of the wing module must satisfy Eq. (4.2).

\subsubsection{Parallel Mechanisms Constraint Methodology}

A full 6-DOF parallel mechanism or robot is free of mechanical constraints, however, as the wing module is a limited mobility mechanism, modifications must be made. To achieve the specific modification, we look again at Eq. (4.1). The physical interpretation of Eq. (4.1) provides the not

only the mobility of the system, but also the number of controllable joints (or actuators/motors) required to control the mechanism. Systems with fewer actuators than system mobility represents an under-actuated system that is unable to provide independent motion for all available degreesof-freedom. Over-actuated systems are the opposite of this in which there are additional actuators than system mobility and results in redundancy in providing independent motion for all available degrees-of-freedom. However, assuming that the system is neither under- nor over-actuated, then the resultant mobility of Eq. (4.1) is also an indicator of the number of constraints (if any) are required to maintain the kinematic integrity of the system. The number of constraints applied to a mechanical system is given by

$$
K=\lambda-M
$$

where $K$ is the number of constraints in the mechanical system. Here, we see that the number of constraints is simply the difference between the system order and mobility of the system.

The morphing wing module that requires at most, four controllable degrees-of-freedom, thus two constraints at a minimum, must be applied to the module in order to maintain the kinematic 
integrity imposed by Eq. (4.3). As the wing module is a specialized parallel-style mechanism, constraints can be added to the system using one of two individual methodologies: $a$ ) introducing a passive branch with specific branch architecture, or b) removing a degree-of-freedom from a joint within the system. These methodologies are distinct and have been applied in various parallel robot systems. For example, a passive branch constraint is used in developing tricept-style parallel robots, whereas joint constraints have been used to develop lower-mobility parallel robots, such as tripod style robots. To further understand the physical implications of each constraint methodology, the mobility and connectivity of each are explored further.

Using Eq. (4.1) and Eq. (4.2), a summary of parallel robot configurations for each constraint methodology are listed in Table 4.1 with respect to mobility and connectivity. In the table, the number of branches, links and joints for configurations ranging from $1 \leq M \leq 6$ are summarized.*.

For the configurations listed in Table 4.1, we see that for every decrease in system mobility, there is a reduction in the number of branches in the system. As previously mentioned, parallel robots in general have an equal number of branches as mobility with a single controllable actuator on each branch. The exception to this is obviously those parallel robots with a passive constraint branch. Regardless of constraint methodology used, the reducing the number of branches within the system cannot be used in a truss-based structure, as removing branches from the system jeopardizes the integrity of the truss itself.

Additionally, it becomes increasingly difficult to generate a suitable parallel mechanism with low system mobility due to the branch connectivity requirements. For the robot to be considered parallel, it must contain at least two branches. We can easily see that each limited mobility mode for the constraint branch methodology satisfies this requirement. However this is not the case for the joint constraint configuration with a mobility $M=1$ as there is only one branch within the system. We also note that the joint constraint configuration with a mobility $M=2$, the two branches within the system are architectural dissimilar to those with a higher mobility. The lack

\footnotetext{
${ }^{*}$ For the passive branch constraint methodology it is assumed that the following link, joint and connectivity parameters are used: $M=6: n_{l}=2$ and $n_{j}=3, M=5: n_{l}=2$ and $n_{j}=3, M=4: n_{l}=2$ and $n_{j}=3$, $M=3: n_{l}=1$ and $n_{j}=2, M=2: n_{l}=1$ and $n_{j}=2, M=1: n_{l}=0$ and $n_{j}=1$
} 
Table 4.1: Mobility and connectivity summary of typical parallel robots

\begin{tabular}{llllllllllll}
\hline \multicolumn{3}{c}{ System } & \multicolumn{3}{c}{ Branch Constraint Parallel Robot } & \multicolumn{3}{c}{ Joint Constraint Parallel Robot } \\
$\mathbf{M}$ & $\mathbf{K}$ & $\mathbf{n}_{\mathbf{b}}$ & $\mathbf{n}_{\mathbf{l}}$ & $\mathbf{n}_{\mathbf{j}}$ & $\sum \mathbf{f}_{\mathbf{j}}$ & $\mathbf{C}_{\mathbf{k}}{ }^{1}$ & $\mathbf{n}_{\mathbf{b}}$ & $\mathbf{n}_{\mathbf{l}}$ & $\mathbf{n}_{\mathbf{j}}$ & $\sum \mathbf{f}_{\mathbf{j}}$ & $\mathbf{C}_{\mathbf{k}}$ \\
\hline 6 & 0 & 7 & 16 & 21 & 42 & $6,6,6,6,6,6,(6)$ & 6 & 14 & 18 & 36 & $6,6,6,6,6,6$ \\
5 & 1 & 6 & 14 & 18 & 35 & $6,6,6,6,6,(5)$ & 5 & 12 & 15 & 29 & $6,6,6,6,5$ \\
4 & 2 & 5 & 12 & 15 & 28 & $6,6,6,6,(4)$ & 4 & 10 & 12 & 22 & $6,6,5,5$ \\
3 & 3 & 4 & 9 & 11 & 21 & $6,6,6,(3)$ & 3 & 8 & 9 & 15 & $5,5,5$ \\
2 & 4 & 3 & 7 & 8 & 14 & $6,6,(2)$ & 2 & 6 & 4 & 8 & 4,4 \\
1 & 5 & 2 & 4 & 4 & 7 & $6,(1)$ & 1 & 2 & 1 & 1 & 1 \\
\hline
\end{tabular}

1 The connectivity of the constraint branch is shown in parentheses

of similarity in branch connectivity makes any comparison between the configurations impossible, as the configurations with a mobility $M>2$ are categorized differently.

The physical ramifications mentioned above render traditional parallel robots unable to fulfill the requirements of a multi-configuration, truss-based mechanism, as which is to be used for the wing module. As such, modifications must be made in order to accommodate the unique requirements of the wing. From Table 4.1, we do see that both systems are entirely dependent on the configuration of particular passive branches. Thus a closer look at the mobility equation is warranted.

First, we can expand Eq. (4.1) to differentiate between the unconstrained branches, and passive constrained branches

$$
M=\lambda\left(n_{l, u}+n_{l, c}-n_{j, u}-n_{j, c}-1\right)+\sum_{j=1}^{n_{j, u}} f_{j, u}+\sum_{j=1}^{n_{j, c}} f_{j, c}
$$

where the subscripts ' $u$ ' and 'c' refer to links and joints situated in unconstrained branches, and passive constrained branches, respectively. In Eq. (4.4), we can see that $\sum_{j=1}^{n_{j}, u} f_{j, u}$ and $\sum_{j=1}^{n_{j}, c} f_{j, c}$ are summations of the connectivity of each of the unconstrained and constrained branches, respectively. Thus Eq. (4.4) can be rewritten in terms of unconstrained and constrained branches

$$
M=n_{b, u}\left[\lambda\left(n_{l, u}-n_{j, u}\right)+C_{u}\right]+n_{b, c}\left[\lambda\left(n_{l, c}-n_{j, c}\right)+C_{c}\right]+\lambda
$$


where $n_{b}$ are the number of branches, and where the subscripts ' $u$ ' and 'c' still refer to links and joints situated in unconstrained branches, and passive constrained branches, respectively. Additionally, the base and platform link have been accounted for within Eq. (4.5), which renders the equation only a function of the branches within the system.

Equation (4.5) describes the mobility of a parallel mechanical system in terms of a collection of constrained and unconstrained branches. This new form of the mobility analysis allows for the parallel mechanical system to be analyzed in a modular fashion, as the branches themselves are viewed as modules that are used within the system. Unconstrained branch modules can be swapped out with suitable branch candidates to form new parallel mechanical systems with different kinematic qualities, without the need to perform additional mobility and connectivity analyses. Constrained branch modules will alter the mobility of the system, however as will be shown below, Eq. (4.5) is versatile and can be for the analysis of both constraint methodology. It will also be used in developing the truss-based mechanisms to drive the morphing wing modules.

\subsection{Wing Module Design Methodology}

In this section, the mobility and connectivity requirements outlined in Section 4.1 are used to develop the specific wing module concepts based on the branch constraint and joint constraint methodology. For each wing module constraint methodology, a detailed mobility and connectivity analysis is presented, followed by the wing module concept. The branch constraint wing module is presented first, followed by the joint constraint wing module. A comparative discussion is then given contrasting the wing module concepts.

\subsubsection{Branch Constraint Wing Module}

\subsubsection{Branch Constraint Mobility Analysis}

Lower mobility parallel robots designed with the branch constraint methodology have a single constraint branch within the system that houses all of the constraints. Thus in Eq. (4.5), $n_{b, c}=1$. The remaining unconstrained branches in the system are of $\mathrm{UP}_{\mathrm{V}} \mathrm{S}$ joint architecture. Thus $n_{l, u}=2$ 
and $n_{j, u}=3$. Additionally, the unconstrained branches each possess a connectivity $C_{u}=6$. Using these values and setting $\lambda=6$, we see that $\lambda\left(n_{l, u}-n_{j, u}\right)+C_{u}=0$, and Eq. (4.5) reduces to

$$
M=6\left(n_{l, c}-n_{j, c}+1\right)+C_{c}
$$

Here, it is plain to see that the mobility of the system is entirely dependent on the constraint branch, and only the constraint branch.

The constraint branch itself can be configured at random such that the required constraints are imposed on the moving platform as per the requirements of the system, however, overly complex configurations should not in general be considered in lieu of ones that are simple. If we then require the branch to be a simple open-loop mechanism, disregarding the base and platform links as they have already been taken into account within the derivation, then we see that every potential branch follows a joint-link-joint sequence, where the total number of joints is always one greater than the number of links, $n_{j, c}=n_{l, c}+1$. This holds for configurations in which all joints are lower-order joints with a single degree-of-freedom, and configurations in which there are a combination of lower and higher-order joints with multiple degrees-of-freedom. Making the substitution, Eq. (4.6) finally becomes

$$
M=C_{c}
$$

Table 4.2: Mobility and connectivity comparison of typical constraint branch parallel robots

\begin{tabular}{llllllllllll}
\hline \multicolumn{3}{c}{ System } & \multicolumn{3}{c}{ Typical Parallel Robot } & \multicolumn{5}{c}{ New Parallel Robot } \\
$\mathbf{M}$ & $\mathbf{K}$ & $\mathbf{n}_{\mathbf{b}}$ & $\mathbf{n}_{\mathbf{l}}$ & $\mathbf{n}_{\mathbf{j}}$ & $\sum \mathbf{f}_{\mathbf{j}}$ & $\mathbf{C}_{\mathbf{k}}{ }^{1}$ & $\mathbf{n}_{\mathbf{b}}$ & $\mathbf{n}_{\mathbf{l}}$ & $\mathbf{n}_{\mathbf{j}}$ & $\sum \mathbf{f}_{\mathbf{j}}$ & $\mathbf{C}_{\mathbf{k}}{ }^{1}$ \\
\hline 6 & 0 & 7 & 16 & 21 & 42 & $6,6,6,6,6,6,(6)$ & 7 & 16 & 21 & 42 & $6,6,6,6,6,6,(6)$ \\
5 & 1 & 6 & 14 & 18 & 35 & $6,6,6,6,6,(5)$ & 7 & 16 & 21 & 41 & $6,6,6,6,6,6,(5)$ \\
4 & 2 & 5 & 12 & 15 & 28 & $6,6,6,6,(4)$ & 7 & 16 & 21 & 40 & $6,6,6,6,6,6,(4)$ \\
3 & 3 & 4 & 9 & 11 & 21 & $6,6,6,(3)$ & 7 & 15 & 20 & 39 & $6,6,6,6,6,6,(3)$ \\
2 & 4 & 3 & 7 & 8 & 14 & $6,6,(2)$ & 7 & 15 & 20 & 38 & $6,6,6,6,6,6,(2)$ \\
1 & 5 & 2 & 4 & 4 & 7 & $6,(1)$ & 7 & 14 & 19 & 37 & $6,6,6,6,6,6,(1)$ \\
\hline
\end{tabular}

\footnotetext{
1 The connectivity of the constraint branch is shown in parentheses
} 
Equation (4.7) shows that the mobility of a parallel robot using a passive constraint branch is a function only of the connectivity of the constraint branch. The mobility is also independent of the number of unconstrained branches in the system insofar as a suitable number of controllable joints are present within the robot system. A comparison of the mobility and connectivity of typical branch constraint and new branch constraint parallel robots is shown in Table 4.2. The same constraint branch configurations for the various lower mobility modes are used here as were used in Table 4.1. We see that now, there are always seven branches within the system, with six unconstrained branch and one constrained branch. This then allows for a truss-based mechanism to be designed to fulfill the requirements of the morphing wing module, as a set number of branches can be incorporated within the system, thus maintaining the integrity of the truss, and thus the structural characteristics of the wing.

\subsubsection{Branch Constraint Wing Module Conceptual Design}

The module is required to assume 15 different kinematic states, thus the configuration of the constraint branch must change according to the required kinematic motions of the particular wing module configuration. As the module can assume any combination of variable sweep, cant, twist and/or span, then the constraint branch must be configured to allow only for these motions, namely one translational and three rotational degrees-of-freedom. The translational degree-of-freedom obviously corresponds to the span-wise motion of the module, and the sweep, cant and twist degrees-of-freedom correspond to the three rotational degrees-of-freedom. The order in which these degrees-of-freedom are applied will result in different constraint kinematics and the options available are discussed further below.

\section{Translation first, then rotations}

For configurations in which translational motion is followed by subsequent rotations, the rotational joints present within the system are in general, located at the platform airfoil. As such, any span motion is always first and is along the module $z$-axis, followed by any subsequent rotations. Figure 4.1 shows an example wing module with a translational total 


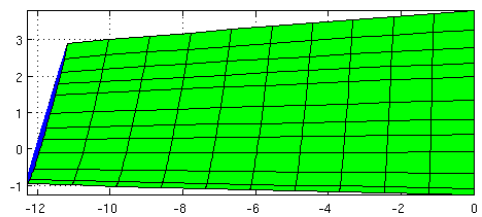

(a) Top View

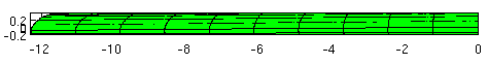

(b) Front View

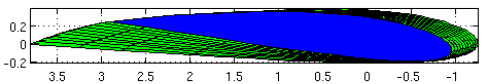

(c) Side View

Figure 4.1: Example of translation then rotation branch constraint module with $15^{\circ}$ sweep, $15^{\circ}$ cant, $-5^{\circ}$ twist, and 12 unit span

span of 12 units, followed by a $15^{\circ}$ sweep, $15^{\circ}$ cant, and $-5^{\circ}$ twist. Here, we see that when the rotations are applied, the module maintains its initial sweep and cant orientations with respect to the aircraft or previous module. Twist motion on the other hand alters the configuration of the module itself. As such, a configuration in which translation is followed by rotation essentially separates the degrees-of-freedom between the module itself, and the subsequent module where sweep and cant motion primarily affect any subsequent module within the wing module sequence. Using this setup for the module, an additional module is always required following a module where sweep and/or cant morphing is required.

\section{Rotations first, then translation}

For configurations in which any rotations are followed by the translation, larger platform airfoil displacements are observed due to the rotational joints being positioned at the base airfoil. This is reflected in Fig. 4.2 in which a $15^{\circ}$ sweep, $15^{\circ}$ cant, and $-5^{\circ}$ twist is followed by a 12 unit span. Here we see that all degrees-of-freedom, including sweep and cant variation, have a direct impact on the orientation of the module itself, which is in contrast to the translation followed by rotation configurations. As such, there is no separation in degrees-offreedom from one module to the next, and there is no requirement that an additional module is needed following a module with sweep and/or cant morphing capabilities.

A summary of the kinematic states and constraint branch architecture for each kinematic option is summarized in Table 4.3. ${ }^{\dagger}$ To facilitate the constraints for the rotational degrees-of-freedom, a transverse revolute joint is used for sweep and cant motion, whereas an axial revolute joint is used

\footnotetext{
${ }^{\dagger}$ For a description of joint types and joint nomenclature, see Table B.1 in Appendix B
} 


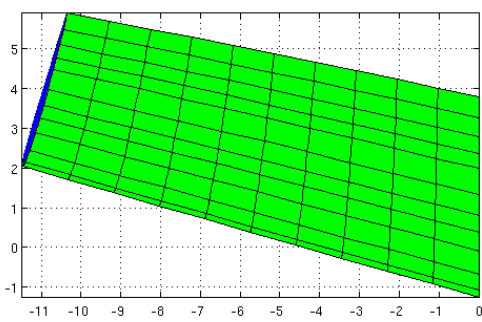

(a) Top View
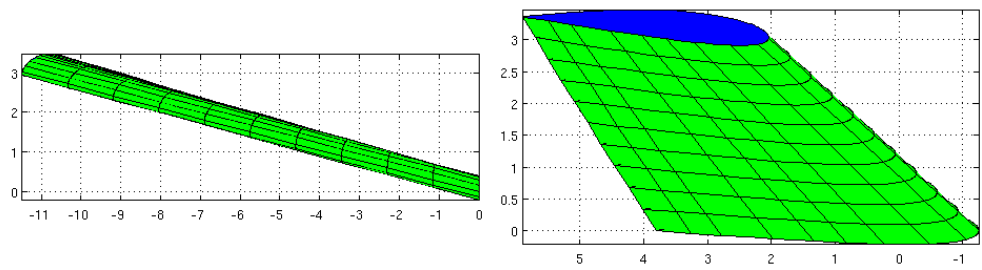

(c) Side View

Figure 4.2: Example of rotation then translation branch constraint module with $15^{\circ}$ sweep, $15^{\circ}$ cant, $-5^{\circ}$ twist, and 12 unit span

Table 4.3: Constraint branch joint sequence summary

\begin{tabular}{|c|c|c|c|c|c|c|}
\hline \multirow{2}{*}{$\begin{array}{l}\text { Module } \\
\text { Mobility }\end{array}$} & \multicolumn{4}{|c|}{ Platform Degrees-of-Freedom } & \multicolumn{2}{|c|}{ Constraint Branch Joint Sequence } \\
\hline & Sweep & Cant & Twist & Span & Trans. $\rightarrow$ Rot. & Rot. $\rightarrow$ Trans. \\
\hline 1 & $\mathrm{X}$ & - & - & - & $\mathrm{R}_{\mathrm{T}}$ & $\mathrm{R}_{\mathrm{T}}$ \\
\hline 1 & - & $\mathrm{X}$ & - & - & $\mathrm{R}_{\mathrm{T}}$ & $\mathrm{R}_{\mathrm{T}}$ \\
\hline 1 & - & - & $\mathrm{X}$ & - & $\mathrm{R}_{\mathrm{A}}$ & $\mathrm{R}_{\mathrm{A}}$ \\
\hline 1 & - & - & - & $\mathrm{X}$ & $\mathrm{P}_{\mathrm{V}}$ & $\mathrm{P}_{\mathrm{V}}$ \\
\hline 2 & $\mathrm{X}$ & $\mathrm{X}$ & - & - & $\mathrm{U}$ & $\mathrm{U}$ \\
\hline 2 & $\mathrm{X}$ & - & $\mathrm{X}$ & - & $\mathrm{R}_{\mathrm{T}} \mathrm{R}_{\mathrm{A}}$ & $\mathrm{R}_{\mathrm{T}} \mathrm{R}_{\mathrm{A}}$ \\
\hline 2 & $\mathrm{X}$ & - & - & $\mathrm{X}$ & $\mathrm{P}_{\mathrm{V}} \mathrm{R}_{\mathrm{T}}$ & $\mathrm{R}_{\mathrm{T}} \mathrm{P}_{\mathrm{V}}$ \\
\hline 2 & - & $\mathrm{X}$ & $\mathrm{X}$ & - & $\mathrm{R}_{\mathrm{T}} \mathrm{R}_{\mathrm{A}}$ & $\mathrm{R}_{\mathrm{T}} \mathrm{R}_{\mathrm{A}}$ \\
\hline 2 & - & $\mathrm{X}$ & - & $\mathrm{X}$ & $\mathrm{P}_{\mathrm{V}} \mathrm{R}_{\mathrm{T}}$ & $\mathrm{R}_{\mathrm{T}} \mathrm{P}_{\mathrm{V}}$ \\
\hline 2 & - & - & $\mathrm{X}$ & $\mathrm{X}$ & $\mathrm{P}_{\mathrm{V}} \mathrm{R}_{\mathrm{A}}\left(\mathrm{R}_{\mathrm{A}} \mathrm{P}_{\mathrm{V}}\right)$ & $\mathrm{R}_{\mathrm{A}} \mathrm{P}_{\mathrm{V}}\left(\mathrm{P}_{\mathrm{V}} \mathrm{R}_{\mathrm{A}}\right)$ \\
\hline 3 & $\mathrm{X}$ & $\mathrm{X}$ & $\mathrm{X}$ & - & $\mathrm{UR}_{\mathrm{A}}$ & $\mathrm{UR}_{\mathrm{A}}$ \\
\hline 3 & $\mathrm{X}$ & $\mathrm{X}$ & - & $\mathrm{X}$ & $\mathrm{P}_{\mathrm{V}} \mathrm{U}$ & $\mathrm{UP}_{\mathrm{V}}$ \\
\hline 3 & $\mathrm{X}$ & - & $\mathrm{X}$ & $\mathrm{X}$ & $\mathrm{P}_{\mathrm{V}} \mathrm{R}_{\mathrm{T}} \mathrm{R}_{\mathrm{A}}$ & $\mathrm{R}_{\mathrm{T}} \mathrm{R}_{\mathrm{A}} \mathrm{P}_{\mathrm{V}}\left(\mathrm{R}_{\mathrm{T}} \mathrm{P}_{\mathrm{V}} \mathrm{R}_{\mathrm{A}}\right)$ \\
\hline 3 & - & $\mathrm{X}$ & $\mathrm{X}$ & $\mathrm{X}$ & $\mathrm{P}_{\mathrm{V}} \mathrm{R}_{\mathrm{T}} \mathrm{R}_{\mathrm{A}}$ & $\mathrm{R}_{\mathrm{T}} \mathrm{R}_{\mathrm{A}} \mathrm{P}_{\mathrm{V}}\left(\mathrm{R}_{\mathrm{T}} \mathrm{P}_{\mathrm{V}} \mathrm{R}_{\mathrm{A}}\right)$ \\
\hline 4 & $\mathrm{X}$ & $\mathrm{X}$ & $\mathrm{X}$ & $\mathrm{X}$ & $\mathrm{P}_{\mathrm{V}} \mathrm{UR}_{\mathrm{A}}$ & $\mathrm{UR}_{\mathrm{A}} \mathrm{P}_{\mathrm{V}}\left(\mathrm{UP}_{\mathrm{V}} \mathrm{R}_{\mathrm{A}}\right)$ \\
\hline
\end{tabular}


for twist motion due to the alignment of rotation axes and coordinate frames. When sweep and cant morphing are present, the combination of the two transverse revolute joints form a universal joint. This is not the case for a combination of sweep and twist or cant and twist as the rotational axes of the combined transverse and axial revolute joint do not form a proper universal joint. Gan et al. $[49,50]$ introduced a reconfigurable Hooke joint that combines one transverse and one axial rotation axis, but for the module, the two joints remain separate. When the module is capable of simultaneous sweep, cant and twist, a universal joint and axial revolute joint are used to impose the necessary constraints. Here, the universal and axial revolute joint could in theory be combined to form a spherical joint as the rotation axes align, however in practice, spherical joints have limited motion ranges about all axes as compared a universal and axial revolute joint combination. For the span-wise translational degree-of-freedom a variable prismatic joint is used. For the configurations in which the prismatic joint is attached to the base or platform airfoil, a fixed prismatic joint could in theory be used, however in regards to space and weight limitations, a fixed prismatic joint imposes a larger penalty as compared to those of the variable prismatic joint. As such, the variable prismatic joint is used when span morphing is required. Lastly, it is worth noting that the span-wise translation axis and twist rotational axis are colinear. As such, when both motions are required within the module, the order of the variable prismatic and axial revolute joint are interchangeable without affecting the translation-rotation sequence. The corresponding constraint branch configurations are shown in parentheses in Table 4.3.

With the various configurations of the constraint branch defined in Table 4.3, the constraint branch wing module is shown kinematically in Fig. 4.4. Here, we see that there are seven branches within the wing module system. Branches 1 through 6 form a specialized truss mechanism that is situated within the bound wing volume as seen in Fig. 4.3(a), and branch 7 is the constraint branch. The unconstrained branches connect to the base and platform airfoils in a loop with three distinct connection points as shown in Fig. 4.3(b). The point $\mathbf{p}_{1}$ lies above the $1 / 4$-chord point on the $x$-axis and $\mathbf{p}_{2}$ lies at the intersection of the camber line and the $y$-axis, with $\mathbf{p}_{3}$ being placed near the lower leading edge depending on the camber (shape) and thickness of the airfoil. Using 
Table 4.4: Enumeration of controllable branch combinations for the constraint branch wing module

\begin{tabular}{|c|c|}
\hline M & Active Branch Combinations \\
\hline 4 & $\begin{array}{l}1-2-3-4,1-2-3-5,1-2-3-6,1-2-3-7,1-3-4-5,1-3-4-6,1-3-4-7,1-4-5-6,1-4-5-7,1-4-6-7, \\
1-5-6-7,2-3-4-5,2-3-4-6,2-3-4-7,2-4-5-6,2-4-5-7,2-5-6-7,3-4-5-6,3-4-5-7,3-4-6-7, \\
4-5-6-7\end{array}$ \\
\hline 3 & $\begin{array}{l}1-2-3,1-2-4,1-2-5,1-2-6,1-2-7,1-3-4,1-3-5,1-3-6,1-3-7,1-4-5,1-4-6,1-4-7,1-5-6, \\
1-5-7,1-6-7,2-3-4,2-3-5,2-3-6,2-3-7,2-4-5,2-4-6,2-4-7,2-5-6,2-5-7,2-6-7,3-4-5, \\
3-4-6,3-4-7,3-5-6,3-5-7,3-6-7,4-5-6,4-5-7,4-6-7,5-6-7\end{array}$ \\
\hline 2 & $\begin{array}{l}1-2,1-3,1-4,1-5,1-6,1-7,2-3,2-4,2-5,2-6,2-7,3-4,3-5,3-6,3-7,4-5,4-6,4-7,5-6, \\
5-7,6-7\end{array}$ \\
\hline 1 & $1,2,3,4,5,6,7$ \\
\hline
\end{tabular}

these connection points results in three planar truss sections within the wing, and are formed by branches 1-2-3, 3-4-5, and 5-6-1. In Fig. 4.3(b), a NACA 4512 airfoil is used to show the rationale for the point selection. Highly cambered airfoils tend to have a lower surface that has very low curvature, thus the planar truss formed by branches 5-6-1, fits this profile well. Also, positioning $\mathbf{p}_{1}$ and $\mathbf{p}_{2}$ on the $x$ and $y$-axes, respectively, give large moment arms for the actuation of cant and sweep morphing, respectively. This is further explained below. Finally, the branch connection point setup leaves sufficient space to include high lift devices such as trailing edge flaps and leading edge slats on the wing [62].

The last step for the conceptual design of the constraint branch wing module is to determine the architecture of the unconstrained branches and the layout of controllable actuators within the module system. While numerous branch candidates are possible (see Appendix B), $\mathrm{UP}_{\mathrm{V}} \mathrm{S}$ style branches are used due to their compact form and resemblance to traditional truss members. Using the branches listed in Table 4.3 for the constraint branch, and the $\mathrm{UP}_{\mathrm{V}} \mathrm{S}$ branches for the unconstrained branches, the allocation of controllable actuators can now be achieved. If each branch can only have one controllable actuator, and the constraint branch can also have a controllable joint, then the list of enumerated module configurations is shown in Table 4.4. At total of 21, 35, 21, and 7 configurations are possible for the 4-DOF, 3-DOF, 2-DOF and 1-DOF modules, respectively. 


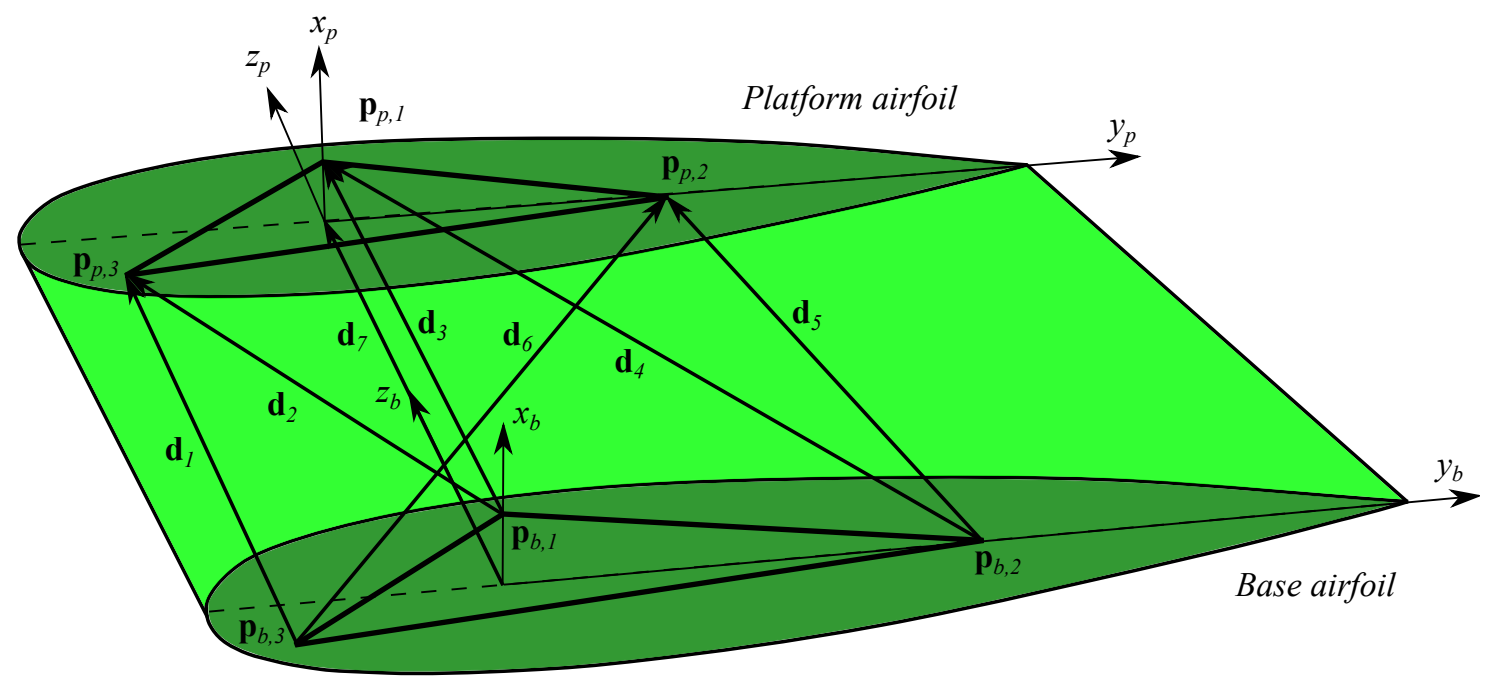

(a) Iso View

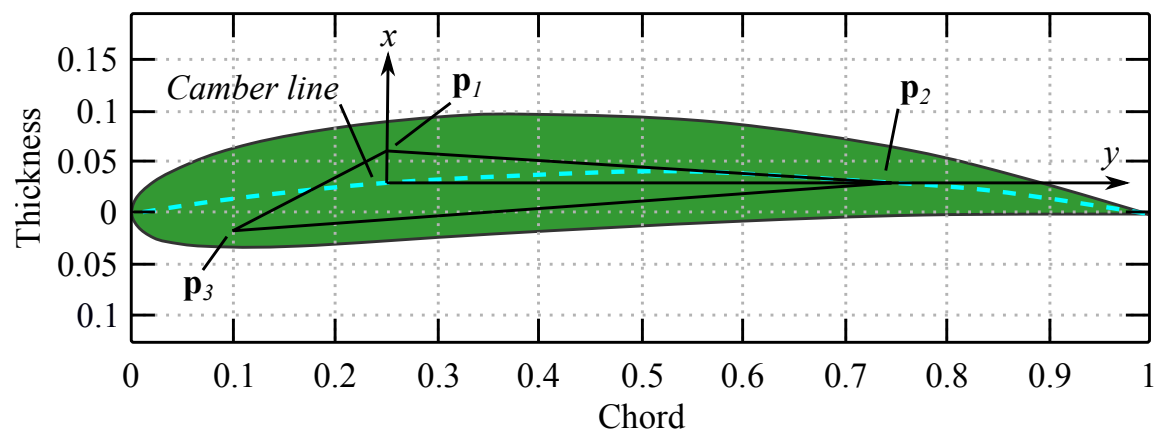

(b) Airfoil View

Figure 4.3: Branch constraint wing module with branch numbers and branch airfoil connection points

To reduce the number of candidate configurations, the single degree-of-freedom modules are analyzed based on the motion required of the platform. The 1-DOF wing modules are shown in Fig. 4.4 where the active branch is shown as a dashed line. Also shown is the required motion of the platform using the translation followed by rotation motion method. For the sweep module shown in Fig. 4.4(a), the rotation of the platform is about the platform $x_{p}$-axis. The branch that provides the largest moment arm for this motion is branch 5, thus for any sweep motion, this branch is designated as active. Similarly for the cant module (Fig. 4.4(b)), the rotation of the platform is about the platform $y_{p}$-axis, thus the branch that provides the larges moment arm and is used for any cant motion is branch 3 . The twist module on the other hand require a rotation about the $z_{p}$-axis. With branches 3 and 6 eliminated from contention, any of branches $1,2,4,6$ or 7 can be 


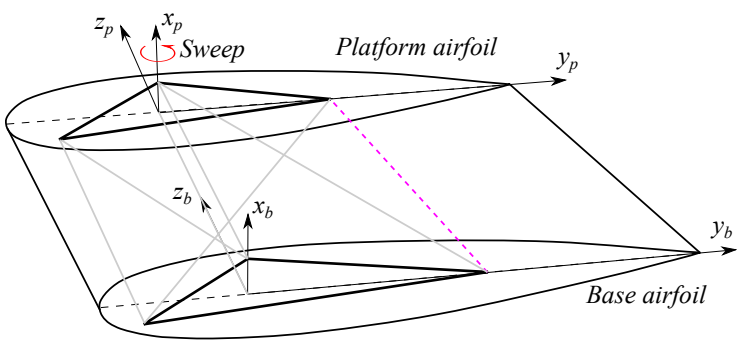

(a) Sweep module

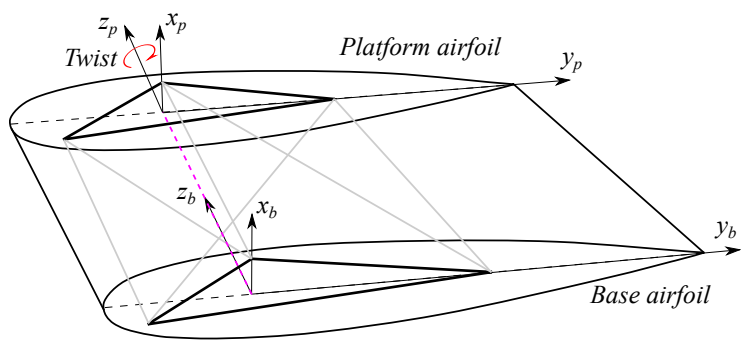

(c) Twist module

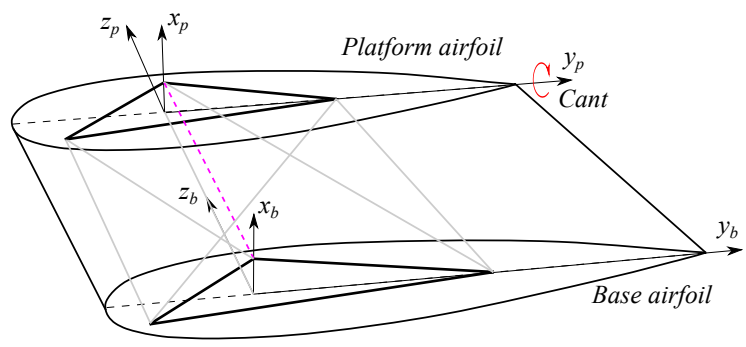

(b) Cant module

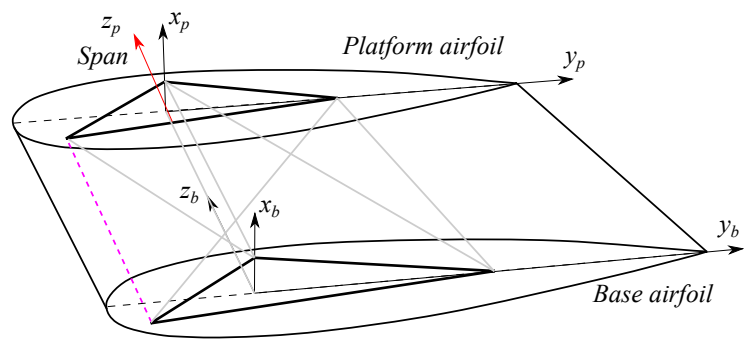

(d) Span module

Figure 4.4: Constraint branch modules with 1-DOF (dashed line represents active branch)

used to provide platform twist. Branch 1 is very closely aligned to the twist axis, thus represent a dead point in regards to actuation, and thus is not suitable to provide twist motion. Branches 2,4 and 6 all are situated at an angle to the twist axis and thus could be used, however, we note that for any configuration which requires twist, there is a revolute joint at the platform on the constraint branch (see Table 4.3). As the $\mathrm{R}_{\mathrm{A}}$ and $z_{p}$-axis align, this joint can be used as an active joint to provide the necessary platform twist, thus designating branch 7 to be used for any configuration which requires twist as shown in Fig. 4.4(a). This leaves branches 1, 2, 4, and 6 available for control of the span motion. As branch 1 is very closely aligned with the span-wise motion of the module, this branch is then designated for all span motion as shown in Fig. 4.4(d). Branches 2, 4, and 6, the diagonally aligned branches, thus remain passive at all times.

Using the distribution of actuators above, modules ranging from 4 to 1-DOF can quickly be configured by either adding or removing the corresponding actuator for the particular motion, while simultaneously reconfiguring the constraint branch. If branches $1,3,5$ and 7 are used exclusively to provide the module with span, sweep, cant and twist morphing capabilities, respectively, then the final configurations for the branch constraint module are listed in Table 4.5. For example, a 
Table 4.5: Enumeration of final configurations for the constraint branch wing module

\begin{tabular}{ll}
\hline $\mathbf{M}$ & Active Branch Combinations \\
\hline 4 & $1-3-5-7$ \\
3 & $1-3-5,1-3-7,1-5-7,3-5-7$ \\
2 & $1-3,1-5,1-7,3-5,3-7,5-7$ \\
1 & $1,3,5,7$ \\
\hline
\end{tabular}

1-3-7 module represents a module capable of variable cant, twist and span. As such, we see that there are 1, 4, 6 and 4 final configurations for the 4-DOF, 3-DOF, 2-DOF and 1-DOF constraint branch wing modules, respectively. These configurations match the number of possible motion combinations shown in Fig. 1.4.

\subsubsection{Joint Constraint Wing Module}

\subsubsection{Joint Constraint Mobility Analysis}

Parallel robots that assume lower mobility modes via joint constraints require that a single degreeof-freedom to be removed from the system for each reduction in mobility from a full 6-DOF robot. Although there is no restriction on where degrees-of-freedom are removed, it is common and practical to impose a requirement that degrees-of-freedom only be removed from branches with a connectivity $C_{k}=6$, resulting in a branch of connectivity $C_{k}=5$. If multiple degrees-of-freedom are removed from a single branch, then the robot more closely resembles the constraint branch parallel robot. Thus to provide sufficient differentiation, degrees-of-freedom can only be removed from a branch of connectivity $C_{k}=6$.

When the robot assumes a limited mobility mode, any unconstrained branch has $C_{u}=6$, $n_{l, u}=2$, and $n_{j, u}=3$ and any constrained branch has $C_{c}=5, n_{l, c}=2$, and $n_{j, c}=2$. Using these values and $\lambda=6$, Eq. (4.5) reduces to

$$
M=6-n_{b, c}
$$

Here we see that the mobility of a joint constraint parallel robot is only dependent on the number 
of constrained branches within the system. Similar to the branch constraint parallel robot, the mobility here is also independent of the number of unconstrained branches in the system insofar as a suitable number of controllable joints are present within the robot system. A comparison of the mobility and connectivity of typical joint constraint and new joint constraint parallel robots is shown in Table 4.6. We see that now, there are always six branches with an equal number of constrained branches as system mobility. This allows allows for configurations with a mobility $M<3$ to be developed without losing the physical resemblance of a parallel robot. Again, similar to the branch constraint parallel robot, the new joint constraint methodology allows for a truss-based mechanism to be designed to fulfill the requirements of the morphing wing module.

\subsubsection{Joint Constraint Wing Module Conceptual Design}

The base design for the joint constraint wing module is shown in Fig. 4.5. Equation (4.8) indicates that an unlimited number of unconstrained branches can exist in the system, and the mobility is only dependent on the number of constrained branches. Here, a constraint branch is any branch that reconfigures from a $\mathrm{UP}_{\mathrm{V}} \mathrm{S}$ to a $\mathrm{R}_{\mathrm{T}} \mathrm{P}_{\mathrm{V}} \mathrm{S}$ configuration. The minimum number of branches as previously developed is six, however a total of eight are used to form a rectangular truss as shown in Fig.4.5. Branches 1 and 3 diagonally span the upper and lower wing, respectively, while branches 2 and 4 are situated in the trailing and leading edge regions, respectively. Branches 5 through 8

Table 4.6: Mobility and connectivity comparison of typical joint constraint parallel robots

\begin{tabular}{|c|c|c|c|c|c|c|c|c|c|c|c|}
\hline \multicolumn{2}{|c|}{ System } & \multicolumn{5}{|c|}{ Typical Parallel Robot } & \multicolumn{5}{|c|}{ New Parallel Robot } \\
\hline M & $\mathbf{R}$ & $\mathbf{n}_{\mathbf{b}}$ & $\mathrm{n}_{1}$ & $\mathbf{n}_{\mathbf{j}}$ & $\sum \mathbf{f}_{\mathbf{j}}$ & $\mathrm{C}_{\mathrm{k}}$ & $\mathbf{n}_{\mathbf{b}}$ & $\mathbf{n}_{1}$ & $\mathbf{n}_{\mathbf{j}}$ & $\sum \mathbf{f}_{\mathbf{j}}$ & $\mathrm{C}_{\mathrm{k}}$ \\
\hline 6 & 0 & 6 & 14 & 18 & 36 & $6,6,6,6,6,6$ & 6 & 14 & 18 & 36 & $6,6,6,6,6,6$ \\
\hline 5 & 1 & 5 & 12 & 15 & 29 & $6,6,6,6,5$ & 6 & 14 & 18 & 35 & $6,6,6,6,6,5$ \\
\hline 4 & 2 & 4 & 10 & 12 & 22 & $6,6,5,5$ & 6 & 14 & 18 & 34 & $6,6,6,6,5,5$ \\
\hline 3 & 3 & 3 & 8 & 9 & 15 & $5,5,5$ & 6 & 14 & 18 & 33 & $6,6,6,5,5,5$ \\
\hline 2 & 4 & 2 & 6 & 4 & 8 & 4,4 & 6 & 14 & 18 & 32 & $6,6,6,5,5,5$ \\
\hline 1 & 5 & 1 & 2 & 1 & 1 & 1 & 6 & 14 & 18 & 31 & $6,5,5,5,5,5$ \\
\hline
\end{tabular}




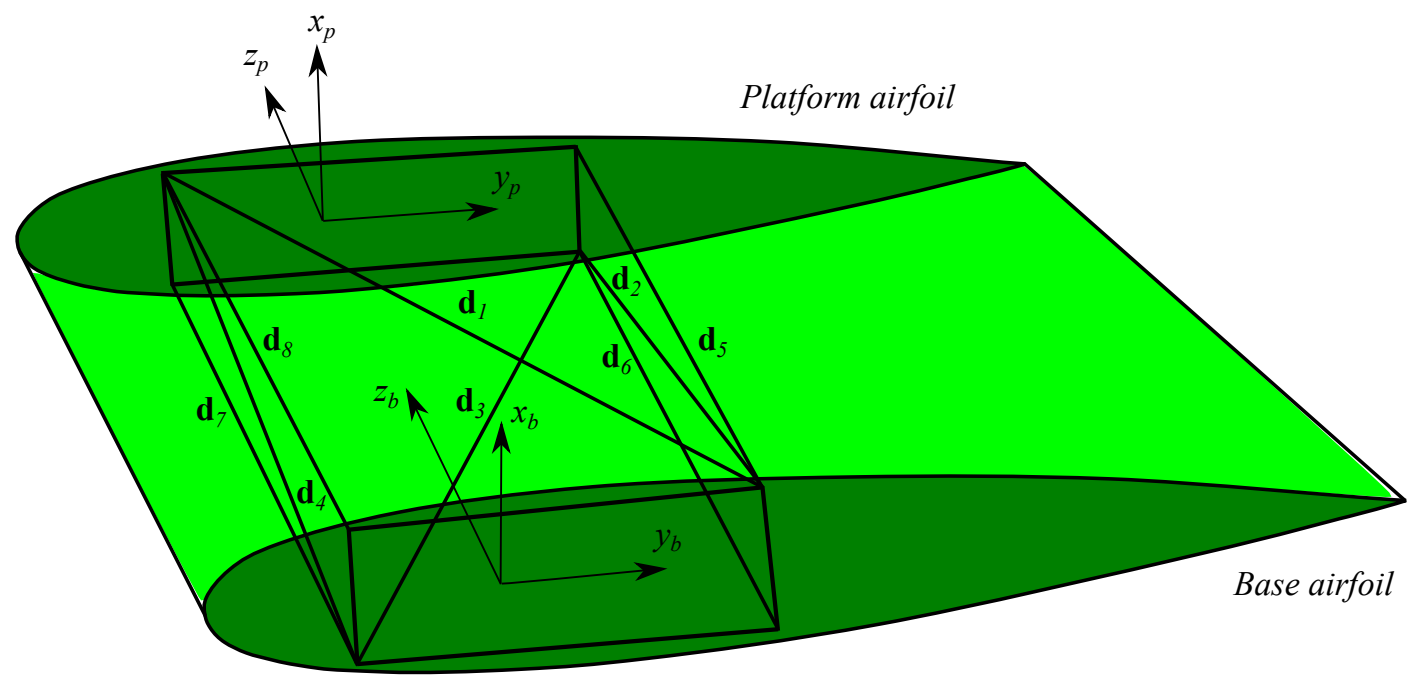

Figure 4.5: Joint constraint wing module

are axial branches with branches 5 and 6 at the trailing region and branches 7 and 8 at the leading region. The four diagonal and four axial branches, or truss members, form a rectangular prism with planar trusses formed around the perimeter of the entire truss mechanism. The planar trusses are formed by the 1-5-8, 2-5-6, 3-6-7, and 4-7-8 branches. The positioning of the leading and trailing planar trusses 4-7-8, and 2-5-6, respectively are located near to the area in which the wing spars would have normally be placed.

From the eight branches, the number of constrained branches that reconfigure from a $\mathrm{UP}_{\mathrm{V}} \mathrm{S}$ to a $\mathrm{R}_{\mathrm{T}} \mathrm{P}_{\mathrm{V}} \mathrm{S}$ configuration is equal to the mobility of the wing module. However, the combination of the constrained branches is independent of the specific degrees-of-freedom the wing module is required to provide. With eight branches within the wing module forming the kinematic truss, multiple configurations can be used for each mobility mode. Additionally, the number of active branches, i.e. those with a controllable degree-of-freedom, is equal to the mobility of the wing module. As with the joint constraint module, only one joint can be active per branch, thus there are an equal number of active branches as wing module mobility. For example, a 4-DOF wing module has four branches designated as being active. With the $\mathrm{UP}_{\mathrm{V}} \mathrm{S} / \mathrm{R}_{\mathrm{T}} \mathrm{P}_{\mathrm{V}} \mathrm{S}$ branch configuration, the prismatic joint is controllable.

From the kinematic requirements of the truss, we can see that a branch can be categorized as 


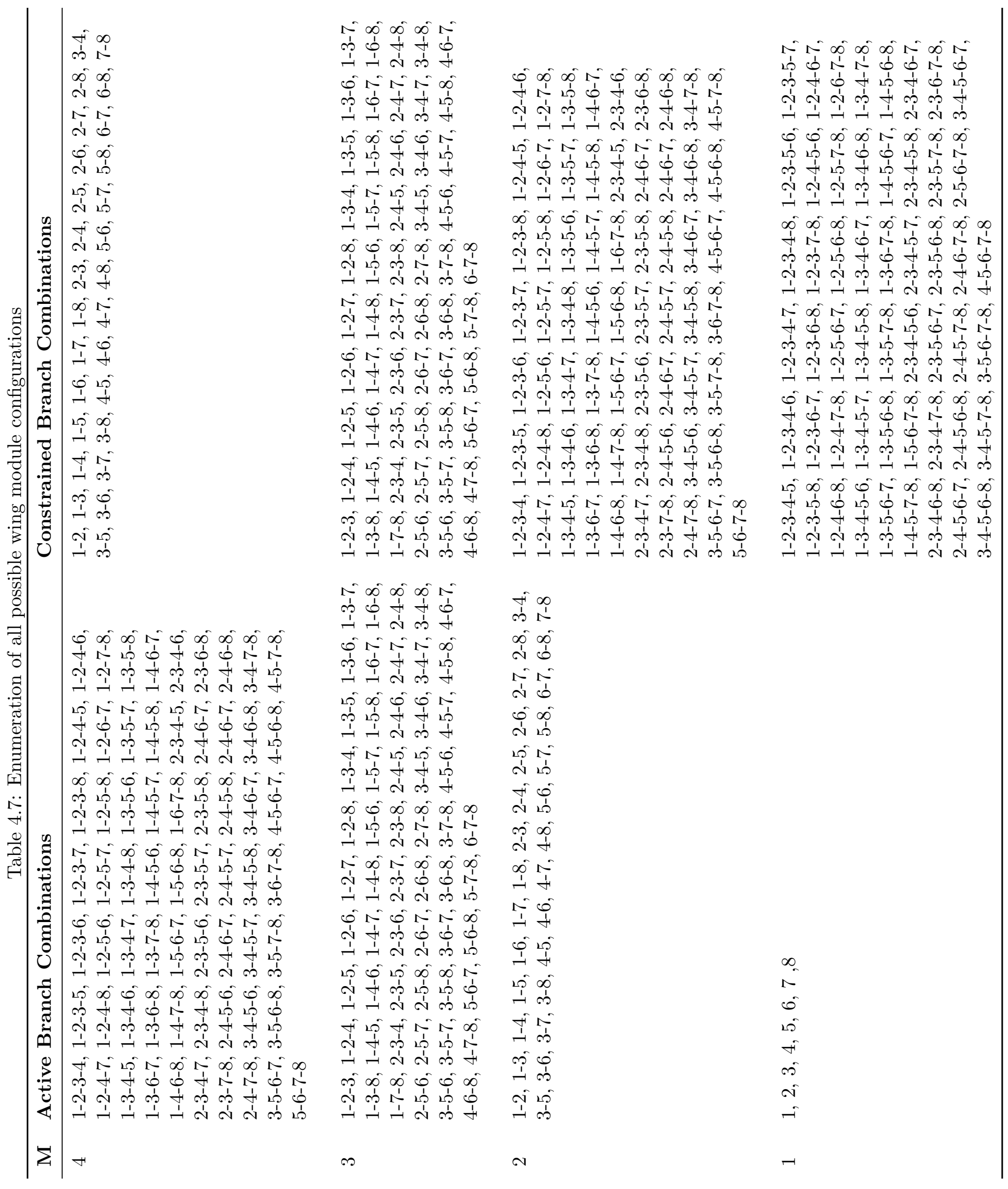


being unconstrained or constrained and as being active or passive. The unconstrained/constrained and active/passive state of the branches are mutually exclusive, thus branches can be either unconstrained-active, unconstrained-passive, constrained-active or constrained-passive. With the eight branches of the wing module labeled in Fig. 4.5, Table 4.7 enumerates the configurations the module can assume for each mobility mode. In the table, configurations are grouped according to the active branches and then passive branches within the system. Each entry in the second column can be combined with an entry in the third column. As such, each configuration is unique. For illustrative purposes, a configuration from each mobility mode is shown in Fig. 4.6 where unconstrained-active branches are solid, unconstrained-passive are dashed, constrained-active are dotted, and constrained-passive are dash-dotted. For example, Fig. 4.6(a) shows 4-DOF module configuration with a 1-2-3-4 active branch and 5-6 passive branch setup. Branches 7 and 8 are unconstrained and passive. In this case there are unconstrained-active, unconstrained-passive and constrained branches are present within the system. Figure 4.6(b) represents a 3-DOF module with 2-3-6 active and 3-5-6 constrained branches. Here unconstrained-active, unconstrained-passive, constrained-active and constrained-passive branches are present within the system. A 2-DOF wing module is shown in Fig. 4.6(c).

In total, we see that for the $4,3,2$, and 1-DOF mobility modes, a total of 1792, 3136, 1792, and 416 unique configurations are possible, respectively. Note here that these configurations are independent of the module sweep, cant, twist or span-morphing capabilities. The configurations listed in Table 4.7 contain a mix of unconstrained-active, unconstrained-passive, constrained-active or constrained-passive branches, which greatly increase the number of enumerated configurations. To help reduce the candidate configurations, a restriction on the active/passive, unconstrained/constrained combinations are imposed. As used in [59,64], branches are limited to be either unconstrained-active, unconstrained-passive, or constrained-passive, thus eliminating constrained-active branches. Unconstrained branches, active and passive, now are of UPS branch architecture with a controllable prismatic joint for those that active, while constrained branches are of RPS branch architecture. This reduces configuration candidates for the 4, 3, 2 and 1-DOF 


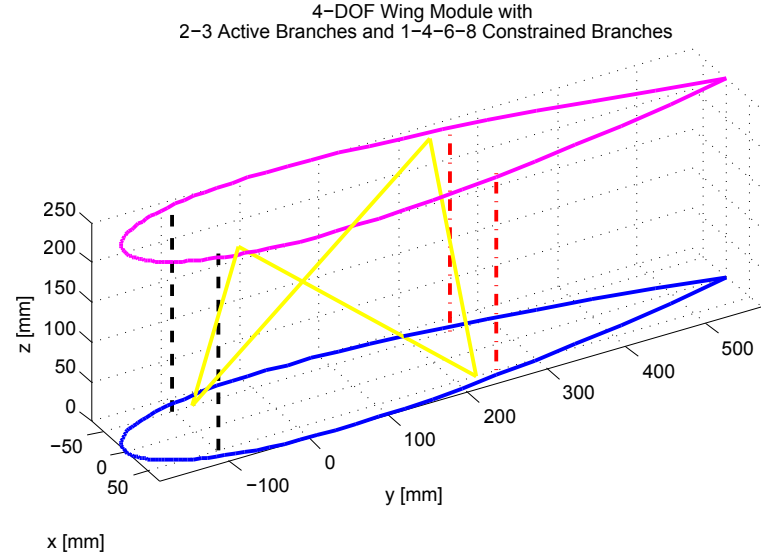

(a) 4-DOF Wing Module

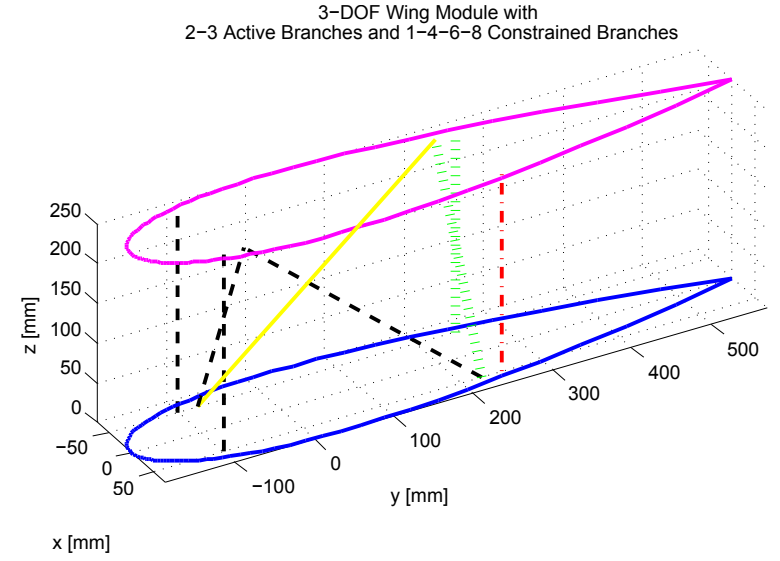

(b) 3-DOF Wing Module

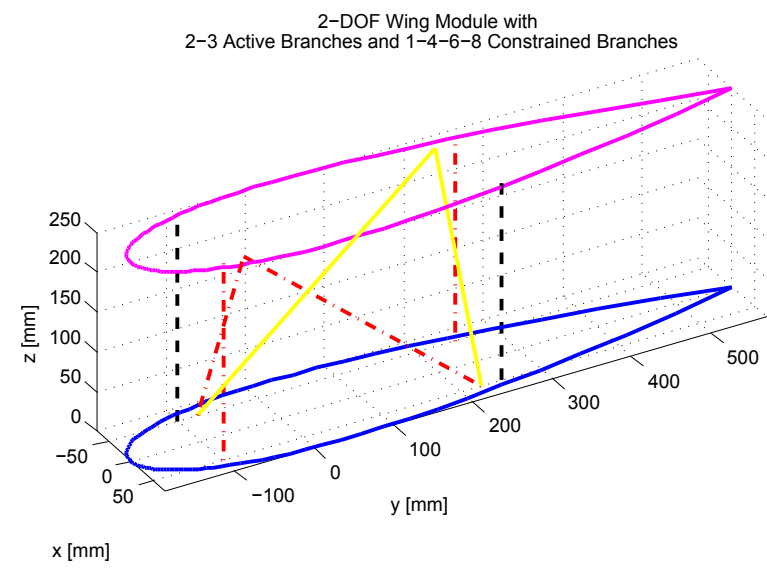

(c) 2-DOF Wing Module

Figure 4.6: Example wing module configurations

mobility modes to a total of $384,560,384$, and 160 unique configurations, respectively.

Finally, we can reduce the number of candidate configurations by limiting active branches to the diagonal truss members, and constrained branches to the axial truss members. This however eliminates the single degree-of-freedom module configurations as there are only four axial truss members and the single degree-of-freedom modules require five constraints. Constraints can in theory be added by removing a degree-of-freedom from elsewhere in the system. There are compelling reasons as to why the single degree-of-freedom modules be discarded which are listed below.

1. First, from a paralle; mechanism perspective, four-bar linkages are sufficient to provide a single degree-of-freedom. The module in the current configuration is overly complicated for 
Table 4.8: Enumeration of final wing module configurations

\begin{tabular}{lll}
\hline $\mathbf{M}$ & Active Branch Combinations & Constrained Branch Combinations \\
\hline 4 & $1-2-3-4$ & $5-6,5-7,5-8,6-7,6-8,7-8$ \\
3 & $1-2-3,1-2-4,1-3-4,2-3-4$ & $5-6-7,5-6-8,5-7-8,6-7-8$ \\
2 & $1-2,1-3,1-4,2-3,2-4,3-4$ & $5-6-7-8$ \\
\hline
\end{tabular}

such a motion set.

2. The resultant motion of the platform airfoil may be highly irregular, and perhaps undesirable. Also, the constrained kinematics could be very difficult or be impossible to solve.

3. One of the requirements of the modular morphing wing is the ability to position degrees-offreedom a specific locations along the wing span. As such, varying the span of each module satisfies this requirement, and allows specific placement of the remaining degrees-of-freedom.

For these reasons, eliminating the single degree-of-freedom modules is justifiable, and thus reduces the candidate configurations to a more reasonable number. Table 4.8 enumerates the candidate configurations that remain. Here we see that there are a total of 6,16 , and 6 unique configurations for the 4, 3, and 2-DOF modules, respectively.

\subsubsection{Module Comparison}

From the architecture descriptions of the two different wing modules, the difference between the two lie not only in the layout of the constraint methodology used, the branch layout, but also in reconfiguration. Much of this chapter was dedicated to describing in detail, the constraint methodology of each wing module. The branch constraint wing module uses a central branch to house all of the constraints, while the joint constraint module distributes constraints amongst the branches.

The branch layout between the two proposed systems are very different. The branch constraint module consisted of six unconstrained branches, with a central constrained branch. The six unconstrained branches formed a triangular prism shaped truss joining the base airfoil to the platform 
airfoil. The joint constraint module on the other hand consisted of eight branches forming a rectangular prism truss with constrained and unconstrained branches distributed amongst the branches. The physical location of the joint constraint branches lie within the volume that would be filled by the wing torque box, thus following a more sensible layout. From Eq. (4.7), we see that the mobility of the branch constraint module is dependent only on the central constrained branch. Thus adding two additional unconstrained branches and altering their layout to one that is identical to the joint constraint module is an option to bring the branch constraint module in line with current wing geometry.

Branch layout of course can be altered, as mentioned, however reconfiguration of each is much different. Reconfiguration of branch constraint module requires removing degrees of freedom from the central branch and thus altering the kinematic topology of joints within the structure. As shown in Table 4.3, the configuration of the module is fully dependent on the degree-of-freedom requirements of the wing module. The joint constraint module, on the other hand, separates the degree-of-freedom requirements from the specific constraint configuration and thus offers much more flexibility in configuring the module. As each of the eight branches within the system are candidates in which a constraint may be placed, multiple configurations can be assumed to perform the same morphing requirements of the module. The kinematic performance of the module can thus be adjusted to not only address the degree-of-freedom requirements of the wing, but also obtain an optimized kinematic performance. It is for this reason that the joint constraint wing module is the more desirable option for the development of the modular morphing wing system.

\subsubsection{Passive Wing Module}

It is worth noting that within the morphing wing system, there may be instances where morphing is not needed. For instance, in the vicinity of the wing tip, kinematic capabilities may not necessarily be required as the wing tip location can be controlled by inboard modules. In these instances, a passive wing module can be utilized in which kinematic capabilities of the wing truss are removed by replacing all prismatic joints with fixed-lengths links. This offers greater flexibility for the modular 
wing system.

\subsection{Summary}

In this chapter, the kinematic architectural requirements of the morphing wing module was presented. The overview of common mechanical systems from the previous chapters was used to provide a basis for the development of the modular morphing wing system. A specialized variable geometry truss system with parallel mechanism modules was proposed for the architectural development process. As the wing module is a constrained system, two parallel mechanism constraint methodologies were proposed for the wing module. A detailed branch-based mobility analysis was developed and branch enumeration evaluation was completed for each module constraint methodology. The mobility analysis provided the branch connectivity requirements for the modules and a concept was proposed for each constraint methodology. A comparison of the two module concepts lead to the selection of the joint constraint model as the base for the wing module. Lastly, the idea of passive modules was discussed.

It should also be noted that the branch enumeration process can easily be used for other modular systems in which volumetric and weight constraints are not as strict as those required for a wing system. Thus, branches that are kinematically feasible but not suitable due to their individual workspace requirements here, may be better suited for other mechanisms, and could provide the means to develop new modular parallel-style mechanisms. These branches are listed in Appendix B. 


\section{Chapter 5}

\section{Wing Module Kinematic Modeling}

In this chapter, the kinematics of the wing module are developed. First, the kinematic setup of the wing module is given including the establishment of branch vector loop kinematics. This is followed by the development of a parametric formulation for the kinematics of the wing module. Here a new parametric constraint system is introduced and applied to the wing module. With the constraint system established, the methods by which to solve the constraints are detailed. This is then followed by the development of the wing module instantaneous kinematics, and the system Jacobian and constrained Jacobian matrices. With the kinematics formulated, the kinematic performance of the wing module is evaluated. Variable range and constraint Jacobian inverse condition distribution are used as indices to measure the kinematic performance of the module for the different module configurations and comments on reconfiguration and configuration selection are given. Finally, a summary of the chapter is given.

\subsection{Wing Module Kinematic Setup}

The joint constraint module, as mentioned in Section 4.2.3 was chosen to be used as the platform in which the morphing wing module was to be built upon. In the previous chapter, the kinematic architecture of the module described in detail and the module joint topology was studied. With the architecture established, the kinematic model of the wing can be developed.

The kinematics of parallel robots, including the wing module, can be modeled using branch 


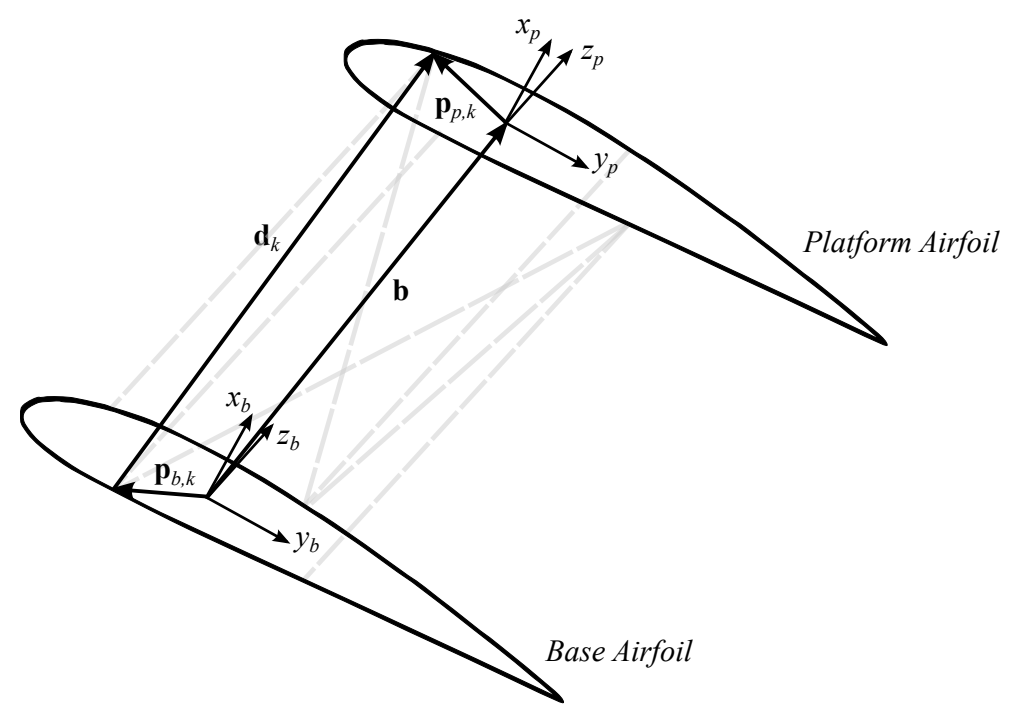

Figure 5.1: Joint constraint wing module shown branch vector loop

loop vectors. The loop vectors trace separate paths within the module, linking a common interior path for all branches, with individual paths for each branch, thus forming a loop of vectors. An example of a vector loop the $k^{t h}$ branch of the wing module is shown in Fig. 5.1. From the figure, we can see that for the $k^{t h}$ branch, the following vector loop equations holds

$$
\mathbf{p}_{b, k}+\mathbf{d}_{k}=\mathbf{b}+\mathbf{p}_{p, k}
$$

where for the $k^{\text {th }}$ branch, $\mathbf{p}_{b, k}$, and $\mathbf{p}_{p, k}$ are the base, and platform airfoil branch vectors, respectively, $\mathbf{d}_{k}$ is the branch vector, and $\mathbf{b}$ is the height of the platform airfoil above the base, and is the span of the module. All of the vectors in Eq. (5.1) are expressed in the base-fixed coordinate frame $\mathcal{O}_{b}$.

If a 6 -DOF parallel robot were to be analyzed using Eq. (5.1), $\mathbf{b}=\left\{\begin{array}{lll}b_{x}, & b_{y}, & b_{z}\end{array}\right\}^{\mathrm{T}}$ and $\mathbf{p}_{p, k}=\mathbf{R}_{x}(\Lambda) \mathbf{R}_{y}(\Gamma) \mathbf{R}_{z}(\alpha) \mathbf{p}_{p, k}^{\prime}$ contain the six controllable degrees-of-freedom. Here $\mathbf{p}_{p, k}^{\prime}=$ $\left\{p_{k, x}^{\prime}, \quad p_{k, y}^{\prime}, \quad p_{k, z}^{\prime}\right\}^{\mathrm{T}}$, which is the local representation of the platform vector and is expressed in the platform-fixed coordinate frame $\mathcal{O}_{p} . \mathbf{R}=\mathbf{R}_{x}(\Lambda) \mathbf{R}_{y}(\Gamma) \mathbf{R}_{z}(\alpha)$ is the rotation matrix that 
expresses $\mathbf{p}_{p, k}^{\prime}$ within the base-fixed coordinate frame $\mathcal{O}_{b}$ where

$$
\mathbf{R}_{x}=\left[\begin{array}{ccc}
1 & 0 & 0 \\
0 & \cos \Lambda & -\sin \Lambda \\
0 & \sin \Lambda & \cos \Lambda
\end{array}\right], \quad \mathbf{R}_{y}=\left[\begin{array}{ccc}
\cos \Gamma & 0 & \sin \Gamma \\
0 & 1 & 0 \\
-\sin \Gamma & 0 & \cos \Gamma
\end{array}\right], \quad \mathbf{R}_{z}=\left[\begin{array}{ccc}
\cos \alpha & -\sin \alpha & 0 \\
\sin \alpha & \cos \alpha & 0 \\
0 & 0 & 1
\end{array}\right]
$$

The rotation order is chosen to correspond with the sequential sweep, cant and twist rotations of the wing. Equation (5.1) can then be rearranged to solve for the only unknown, the branch vector length

$$
d_{k}=\left|\mathbf{b}+\mathbf{p}_{p, k}-\mathbf{p}_{b, k}\right|
$$

Equation (5.2) can be solved automatically for a 6-DOF parallel robot system as the platform height $\mathbf{b}$ and angles within the platform rotation $\mathbf{R}$ are defined by the user for the particular task. The morphing wing module, however, is a reconfigurable system in which different limited mobility modes can be assumed. Any system operating in a limited mobility state requires constraints to be solved, thus maintaining the kinematic integrity of the system. As outlined in the Chapter 4, with each degree-of-freedom removed from the full 6-DOF system, an additional constraint must be added. The constraints are mathematical equations that describe the physical constraints of the system, which for the wing module, is the motion of the platform. The dependent platform motions, $\mathbf{q}_{D}$ are expressed in terms of the remaining independent motions, $\mathbf{q}_{I}$, such that

$$
\mathbf{q}_{D}=f\left(\mathbf{q}_{I}\right)
$$

Equation (5.3) is the symbolic representation of the system constraints, and specific constraint equations are modeled for the particular mechanism state. As the wing module is capable of task-based reconfiguration, the constraints on the system must change accordingly to not only the current module mobility, but also the configuration of the wing module with respect to the selection of constrained branches. A parametric constraint system must therefore be developed for the wing module that addresses these changes. 


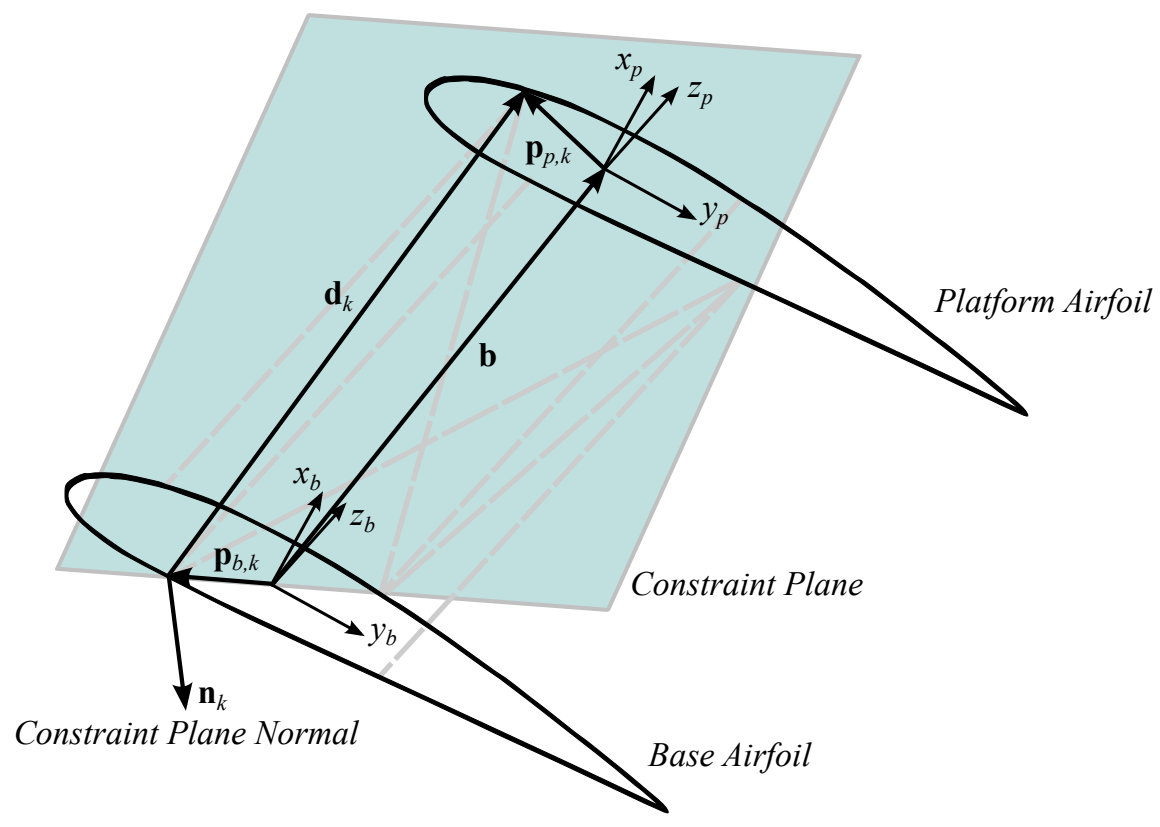

Figure 5.2: Joint constraint wing module shown branch vector loop and constraint plane

\subsection{Module Kinematic Formulation}

In this section, the kinematics of the wing module are developed. First, a new parametric constraint equation is formulated for the wing module. Using the parametric formulation, constraint equation solutions are developed for the mobility modes assumed by the wing module. Afterwards, the instantaneous kinematics of the wing are introduced, including formation of the system Jacobian. Then, the parametric constraint equation is used to form a parametric constrained velocity equation. Finally, the constrained Jacobian is formulated.

\subsubsection{Parametric Constraint Formulation}

In order to solve Eq. (5.2) for each branch within the wing module, the constraints of the system must first be solved. As mentioned, these constraints are mathematical constraints that define the motion of any constrained branch within the system. As the combination of branches, including how many are needed can vary according to mobility requirements of the system, the formulation should be independent of system mobility, and the number and combination of constrained branches.

As mentioned, the spherical joint of each passive branch is constrained to move in a plane as 
shown in Fig. 5.2. From this we can define the following parametric constraint equation

$$
\left(\mathbf{b}+\mathbf{p}_{p, k}\right) \cdot \mathbf{n}_{k}=0
$$

where $\mathbf{n}_{k}$ is the constraint plane normal for branch $k$. Since the constrained branch consists of a RPS joint configuration, $\mathbf{n}_{k}$ is parallel to the rotation axis of the revolute joint at the base. Note that there is no restriction on the the orientation of the constraint plane. Traditionally, for parallel robots with constrained branches, the constraint plane passes through the origin of the base coordinate frame $\mathbf{O}_{b}$ and is perpendicular to the base of the robot, such as the formulation developed by Xi et al. [56] No such requirement is placed on Eq. (5.4), as any orientation can be evaluated. Equation 5.4 is thus a fully parametric constraint equation.

If there are $n_{b, c}$ constrained branches, then Eq. (5.4) can be expanded to included all constrained branches

$$
\left[\begin{array}{cccc}
\left(\mathbf{b}+\mathbf{p}_{p, 1}\right)^{\mathrm{T}} & \mathbf{0}_{1 \times 3} & \cdots & \mathbf{0}_{1 \times 3} \\
\mathbf{0}_{1 \times 3} & \left(\mathbf{b}+\mathbf{p}_{p, 2}\right)^{\mathrm{T}} & \ddots & \vdots \\
\vdots & \ddots & \ddots & \mathbf{0}_{1 \times 3} \\
\mathbf{0}_{1 \times 3} & \cdots & \mathbf{0}_{1 \times 3} & \left(\mathbf{b}+\mathbf{p}_{p, n_{b, c}}\right)^{\mathrm{T}}
\end{array}\right]\left\{\begin{array}{c}
\mathbf{n}_{1} \\
\mathbf{n}_{2} \\
\vdots \\
\mathbf{n}_{n_{b, c}}
\end{array}\right\}=\left\{\begin{array}{c}
0 \\
0 \\
\vdots \\
0
\end{array}\right\}_{n_{b, c} \times 1}
$$

Equation (5.5) forms the system of equations for $n_{b, c}$ constrained branches and grants the required flexibility in selecting the combination and number of constrained branches, and is also independent of the wing module degrees-of-freedom chosen as independent and which are dependent.

For example, a 5-6 type 4-DOF wing module, requires two constrained branches. Applying these constraints to Eq. (5.5) results in the following system of equations

$$
\left[\begin{array}{cc}
\left(\mathbf{b}+\mathbf{p}_{p, 5}\right)^{\mathrm{T}} & \mathbf{0}_{1 \times 3} \\
\mathbf{0}_{1 \times 3} & \left(\mathbf{b}+\mathbf{p}_{p, 6}\right)^{\mathrm{T}}
\end{array}\right]\left\{\begin{array}{l}
\mathbf{n}_{5} \\
\mathbf{n}_{6}
\end{array}\right\}=\left\{\begin{array}{l}
0 \\
0
\end{array}\right\}
$$

This new parametric formulation offers a very high level of flexibility in which task-based reconfiguration is possible $[59,64]$. For the wing module, task-based reconfiguration refers to configuring the module to the required morphing degrees-of-freedom. The particular task therefore dictates 
the mobility of the system and the independent platform airfoil motions. In the section that follows, solutions to the constraint equations are shown for the mobility modes assumed by the wing module. For each developed solution, only one set of module degrees-of-freedom are shown, while the other degree-of-freedom combinations can be derived in a similar manner.

\subsubsection{Constraint Solving}

The particular solution to Eq. (5.5) is dependent on the mobility of the wing module, and the selected constrained branches. If we look at a single constrained branch, and expand Eq. (5.4), the following constraint equation is formed. For simplicity, $\mathbf{p}_{p, k}^{\prime}$ is replaced with $\mathbf{p}_{k}^{\prime}$ to represent the platform airfoil branch vectors since the base airfoil branch vector, $\mathbf{p}_{b, k}$, does not appear in the constraint solution. Thus, expanding Eq. (5.4) yields

$$
\begin{aligned}
n_{k, x}\left(b_{x}+R_{11} p_{k, x}^{\prime}+R_{12} p_{k, y}^{\prime}+R_{13} p_{k, z}^{\prime}\right) & +n_{k, y}\left(b_{y}+R_{21} p_{k, x}^{\prime}+R_{22} p_{i, y}^{\prime}+R_{23} p_{i, z}^{\prime}\right) \\
& +n_{k, z}\left(b_{z}+R_{31} p_{k, x}^{\prime}+R_{32} p_{k, y}^{\prime}+R_{33} p_{k, z}^{\prime}\right)=0
\end{aligned}
$$

where the components of $\mathbf{R}$ are

$$
\begin{array}{lll}
R_{11}=\cos \Gamma \cos \alpha & R_{12}=-\cos \Gamma \sin \Lambda & R_{13}=\sin \Gamma \\
R_{21}=\sin \Lambda \sin \Gamma \cos \alpha+\cos \Lambda \sin \alpha & R_{22}=-\sin \Lambda \sin \Gamma \sin \alpha+\cos \Lambda \cos \Lambda & R_{23}=\sin \Lambda \sin \Gamma \\
R_{31}=-\cos \Lambda \sin \Gamma \cos \alpha+\sin \Lambda \sin \alpha & R_{32}=\cos \Lambda \sin \Gamma \sin \alpha+\sin \Lambda \cos \alpha & R_{33}=\cos \Lambda \cos \Gamma
\end{array}
$$

Using Eq. (5.6), the solution to the particular constraint system can be solved. For each case, $b_{x}$ and $b_{y}$ are mandatory dependent motions. Additional dependent motions for the 3-DOF and 2-DOF module configurations will obviously vary accordingly. Within Fig. 5.3, Fig. 5.4 and Fig. 5.5 examples of module kinematic motions are shown. The base and platform airfoil vectors outlined

in Table 5.1 are used for the module configurations shown within the figures, however operation limits of the joints are disregarded here. Branches that are active-diagonal are dotted lines, passivediagonal are dashed lines, constrained-axial branches are dash-dotted lines, and unconstrained-axial are solid lines. A comparison of the kinematic performance is discussed later in Seciton 5.3. 


\subsubsection{Constraint Solution for 4-DOF Configurations}

For the 4-DOF wing module, there are six unique configurations the module can assume. For this configuration, the module span, sweep, cant, and twist are all independent. The dependent motions

are defined as $\mathbf{q}_{D}=\left\{b_{x}, \quad b_{y}\right\}^{\mathrm{T}}=f\left(b_{z}, \Lambda, \Gamma, \alpha\right)$, thus two constraint equations are required. Using the 5-6 constrained branch configuration as an example, a linear system of equations is formed using Eq. (5.6), and the two constrained motions can be solved as

$$
\left\{\begin{array}{l}
b_{x} \\
b_{y}
\end{array}\right\}=\left[\begin{array}{ll}
n_{x, 5} & n_{y, 5} \\
n_{x, 6} & n_{y, 6}
\end{array}\right]^{-1}\left\{\begin{array}{l}
-\mathbf{p}_{5} \cdot \mathbf{n}_{5}-n_{z, 5} b_{z} \\
-\mathbf{p}_{6} \cdot \mathbf{n}_{6}-n_{z, 6} b_{z}
\end{array}\right\}
$$

\subsubsection{Constraint Solution for 3-DOF Configurations}

For the 3-DOF wing module, 16 unique configurations can be assumed, with four unique constrained branch combinations. In addition to $b_{x}$ and $b_{y}$ being designated as dependent, one platform airfoil rotation must be added to $\mathbf{q}_{D}$. As the selection of constrained rotation is independent of the selection of constrained branches, a total of 12 unique constrained motion and branch combinations are possible. If the enumerated active branches are also included, a total of 48 modules are possible, however, active branches are independent of constrained branches and are not considered. Here, three constraint equations are required. For the developed solution, the module twist, $\alpha$, is designated as dependent, thus $\mathbf{q}_{D}=\left\{b_{x}, \quad b_{y}, \alpha\right\}^{\mathrm{T}}=f\left(b_{z}, \Lambda, \Gamma\right)$, and the 5-6-7 constrained branch configuration is used. The first step is to rearrange Eq. (5.6) in terms of the $\cos \alpha$, $\sin \alpha$, and $b_{x}$ and $b_{y}$. For simplicity, c and s will be used in lieu of cos and sin, respectively. For each constrained branch, collecting forms

$$
\begin{aligned}
0 & =\left[n_{k, x} p_{k, x}^{\prime} \mathrm{c}_{\Gamma}+n_{k, y}\left(p_{k, x}^{\prime} \mathrm{s}_{\Lambda} \mathrm{s}_{\Gamma}+p_{k, y}^{\prime} \mathrm{c}_{\Lambda}\right)+n_{k, z}\left(-p_{k, x}^{\prime} \mathrm{c}_{\Lambda} \mathrm{s}_{\Gamma}+p_{k, y}^{\prime} \mathrm{s}_{\Lambda}\right)\right] \mathrm{c}_{\alpha} \\
& +\left[-n_{k, x} p_{k, y}^{\prime} \mathrm{c}_{\Gamma}+n_{k, y}\left(p_{k, x}^{\prime} \mathrm{c}_{\Lambda} \mathrm{s}_{\Gamma}-p_{k, y}^{\prime} \mathrm{s}_{\Lambda} \mathrm{s}_{\Gamma}\right)+n_{k, z}\left(p_{k, x}^{\prime} \mathrm{s}_{\Lambda} \mathrm{s}_{\Gamma}+p_{k, y}^{\prime} \mathrm{c}_{\Lambda} \mathrm{s}_{\Gamma}\right)\right] \mathrm{s}_{\alpha} \\
& +\left(n_{k, x}\right) b_{x}+\left(n_{k, y}\right) b_{y}+\left[n_{k, x} p_{k, z}^{\prime} \mathrm{s}_{\Gamma}-n_{k, y} p_{k, z}^{\prime} \mathrm{s}_{\Lambda} \mathrm{c}_{\Gamma}+n_{k, z}\left(b_{z}+p_{k, z}^{\prime} \mathrm{c}_{\Lambda} \mathrm{c}_{\Gamma}\right)\right]
\end{aligned}
$$




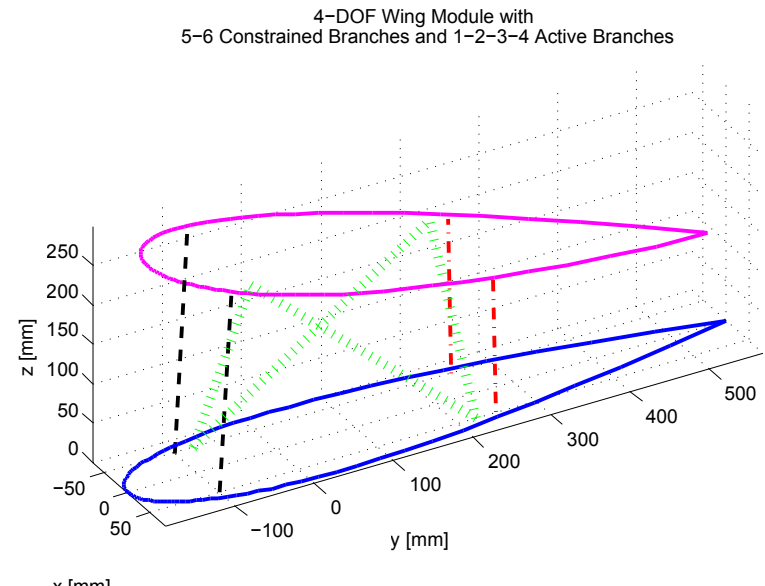

$\mathrm{x}[\mathrm{mm}]$

(a) 5-6 Constrained Branches

4-DOF Wing Module with
5-8 Constrained Branches and 1-2-3-4 Active Branches

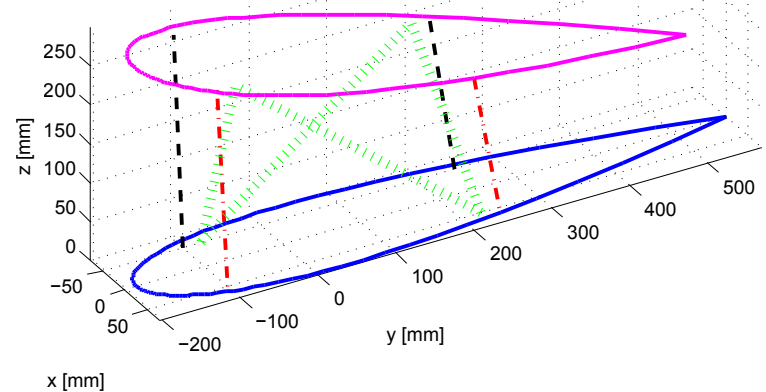

(c) 5-8 Constrained Branches

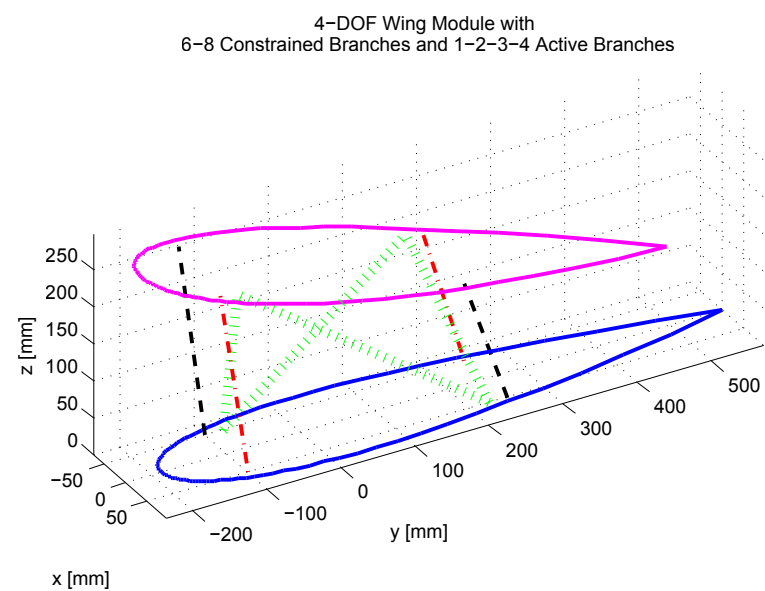

(e) 6-8 Constrained Branches
$4-D O F$ Wing Module with

5-7 Constrained Branches and 1-2-3-4 Active Branches

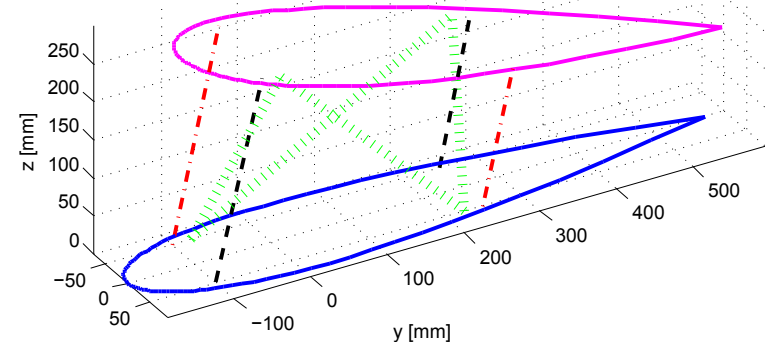

$\mathrm{x}[\mathrm{mm}]$

(b) 5-7 Constrained Branches

4-DOF Wing Module with
6-7 Constrained Branches and 1-2-3-4 Active Branches

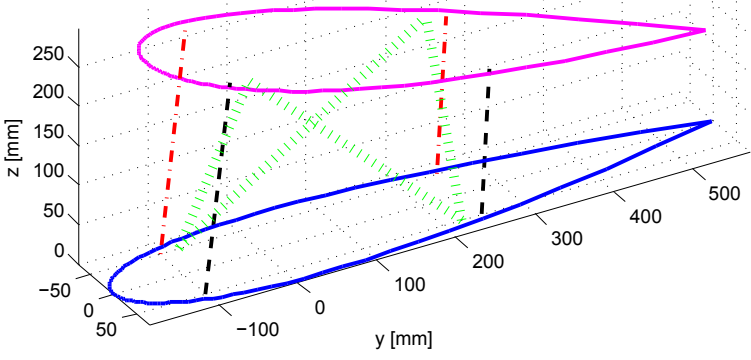

$\mathrm{x}[\mathrm{mm}]$

(d) 6-7 Constrained Branches

4-DOF Wing Module with

7-8 Constrained Branches and 1-2-3-4 Active Branches

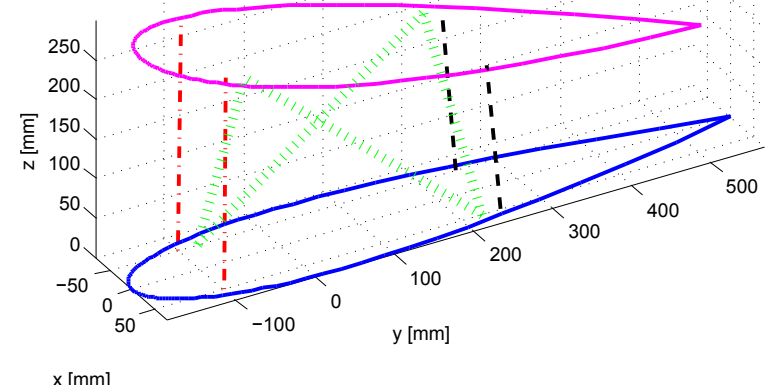

(f) 7-8 Constrained Branches

Figure 5.3: 4-DOF wing modules with red constrained branches and $\Lambda=-15^{\circ}, \Gamma=30^{\circ}, \alpha=-5^{\circ}$ and $b_{z}=275 \mathrm{~mm}$ 
which for the 5-6-7 constrained branch configuration, form the following system of equations

$$
\begin{aligned}
& A_{5} \mathrm{c}_{\alpha}+B_{5} \mathrm{~s}_{\alpha}+n_{5, x} b_{x}+n_{5, y} b_{y}+C_{5}=0 \\
& A_{6} \mathrm{c}_{\alpha}+B_{6} \mathrm{~s}_{\alpha}+n_{6, x} b_{x}+n_{6, y} b_{y}+C_{6}=0 \\
& A_{7} \mathrm{c}_{\alpha}+B_{7} \mathrm{~s}_{\alpha}+n_{7, x} b_{x}+n_{7, y} b_{y}+C_{7}=0
\end{aligned}
$$

Equation (5.10) is nonlinear due to the trigonometric terms, however a numerical solution can still be developed. First, one equation is rearranged to isolate $b_{x}$ which is then substituted into the other two equations. If branch 5 is used to rearrange for $b_{x}$, then the equations for branches 6 and 7 become

$$
\begin{aligned}
& \left(n_{5, x} A_{6}-n_{6, x} A_{5}\right) \mathrm{c}_{\alpha}\left(n_{5, x} B_{6}-n_{6, x} B_{5}\right) \mathrm{s}_{\alpha}+\left(n_{5, x} n_{6, y}-n_{6, x} n_{5, y}\right) b_{y}+\left(n_{5, x} C_{6}-n_{6, x} C_{5}\right)=0 \\
& \left(n_{5, x} A_{7}-n_{7, x} A_{5}\right) \mathrm{c}_{\alpha}\left(n_{5, x} B_{7}-n_{7, x} B_{5}\right) \mathrm{s}_{\alpha}+\left(n_{5, x} n_{7, y}-n_{7, x} n_{5, y}\right) b_{y}+\left(n_{5, x} C_{7}-n_{7, x} C_{5}\right)=0
\end{aligned}
$$

Next, the equations in Eq. (5.11) are rearranged to isolate $b_{y}$, which are then equated to each other, leading to

$$
\begin{aligned}
\left(\frac{n_{5, x} A_{6}-n_{6, x} A_{5}}{n_{5, x} n_{6, y}-n_{6, x} n_{5, y}}-\frac{n_{5, x} A_{7}-n_{7, x} A_{5}}{n_{5, x} n_{7, y}-n_{7, x} n_{5, y}}\right) \mathrm{c}_{\alpha} & +\left(\frac{n_{5, x} B_{6}-n_{6, x} B_{5}}{n_{5, x} n_{6, y}-n_{6, x} n_{5, y}}-\frac{n_{5, x} B_{7}-n_{7, x} B_{5}}{n_{5, x} n_{7, y}-n_{7, x} n_{5, y}}\right) \mathrm{s}_{\alpha} \\
& +\left(\frac{n_{5, x} C_{6}-n_{6, x} C_{5}}{n_{5, x} n_{6, y}-n_{6, x} n_{5, y}}-\frac{n_{5, x} C_{7}-n_{7, x} C_{5}}{n_{5, x} n_{7, y}-n_{7, x} n_{5, y}}\right)=0
\end{aligned}
$$

which is in the form

$$
A^{*} \mathrm{c}_{\alpha}+B^{*} \mathrm{~s}_{\alpha}+C^{*}=0
$$

At this point, Eq (5.13) consists of only one unknown, which is the module twist, $\alpha$. Although multiple solutions to Eq (5.13) exist, a minimum-norm solution can be determined. Equation (5.13) is rearranged into quadratic form in terms of cos and sin

$$
\begin{aligned}
& \left(A^{* 2}+B^{* 2}\right) \cos ^{2} \alpha-2 A^{*} C^{*} \cos \alpha+\left(C^{* 2}-B^{* 2}\right)=0 \\
& \left(A^{* 2}+B^{* 2}\right) \sin ^{2} \alpha-2 B^{*} C^{*} \sin \alpha+\left(C^{* 2}-A^{* 2}\right)=0
\end{aligned}
$$




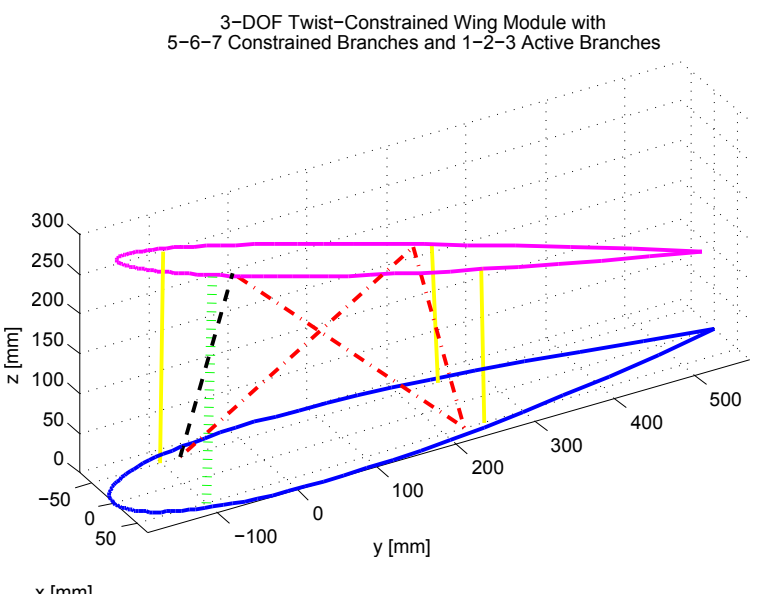

(a) 5-6-7 Constrained Branches

3-DOF Twist-Constrained Wing Module with 5-7-8 Constrained Branches and 1-2-3 Active Branches

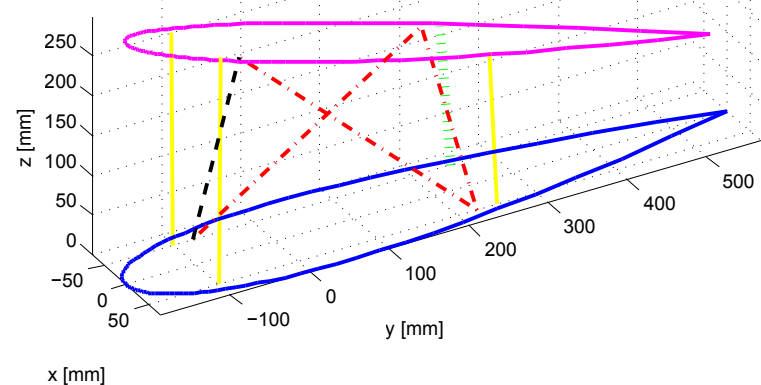

(c) 5-7-8 Constrained Branches
3-DOF Twist-Constrained Wing Module with
5-6-8 Constrained Branches and 1-2-3 Active Branches

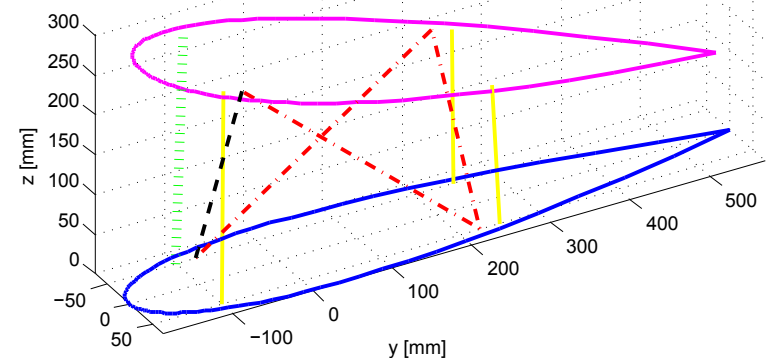

$\mathrm{x}[\mathrm{mm}]$

(b) 5-6-8 Constrained Branches

3-DOF Twist-Constrained Wing Module with 6-7-8 Constrained Branches and 1-2-3 Active Branches

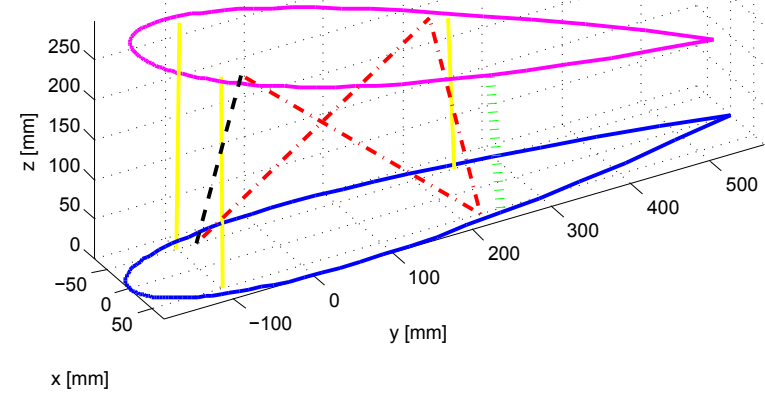

(d) 6-7-8 Constrained Branches

Figure 5.4: 3-DOF twist-constrained wing modules with red constrained branches and $\Lambda=-15^{\circ}$, $\Gamma=30^{\circ}$, and $b_{z}=275 \mathrm{~mm}$

for which each equation, $\alpha$ can be determined

$$
\begin{aligned}
& \alpha=\arccos \left[\frac{2 A^{* 2} C^{* 2} \pm \sqrt{4 A^{* 4} C^{* 4}-4\left(A^{* 2}+B^{* 2}\right)\left(C^{* 2}-B^{* 2}\right)}}{2\left(A^{* 2}+B^{* 2}\right)}\right] \\
& \alpha=\arcsin \left[\frac{2 B^{* 2} C^{* 2} \pm \sqrt{4 B^{* 4} C^{* 4}-4\left(A^{* 2}+B^{* 2}\right)\left(C^{* 2}-A^{* 2}\right)}}{2\left(A^{* 2}+B^{* 2}\right)}\right]
\end{aligned}
$$

The two solutions for each form of Eq. (5.15) are then compared to find one that matches for both the cos and sin quadratic solutions. Alternatively, the minimum norm solution can be found by 
using the generalized inverse as shown in [56]

$$
\left\{\begin{array}{c}
\cos \alpha \\
\sin \alpha
\end{array}\right\}=\frac{1}{A^{* 2}+B^{* 2}}\left\{\begin{array}{l}
A^{*} C^{*} \\
B^{*} C^{*}
\end{array}\right\}
$$

The cos and sin solutions from Eq. (5.16) can then be used to find $\alpha$. After some simplification

$$
\alpha=\arctan \frac{-A^{*}}{B^{*}}
$$

With $\alpha$ known, the remaining dependent motions, $b_{x}$ and $b_{y}$ can now be solved using Eq. (5.10). A similar solution can be obtained using sweep or cant as a constrained motion, and any combination of constrained branches.

Alternatively, if the span of the module is not required, then the solution for the dependent motions defined by $\mathbf{q}_{D}=\left\{b_{x}, \quad b_{y}, \quad b_{z}\right\}^{\mathrm{T}}=f(\Lambda, \Gamma, \alpha)$ can be solved using a system of linear equations similar to Eq. (5.8). If the 5-6-7 constrained branch is therefore used as an example, the constraints are solved as

$$
\left\{\begin{array}{l}
b_{x} \\
b_{y} \\
b_{z}
\end{array}\right\}=\left[\begin{array}{ccc}
n_{x, 5} & n_{y, 5} & n_{y, 6} \\
n_{x, 6} & n_{y, 6} & n_{z, 6} \\
n_{x, 7} & n_{y, 7} & n_{z, 7}
\end{array}\right]^{-1}\left\{\begin{array}{l}
-\mathbf{p}_{5} \cdot \mathbf{n}_{5} \\
-\mathbf{p}_{6} \cdot \mathbf{n}_{6} \\
-\mathbf{p}_{7} \cdot \mathbf{n}_{7}
\end{array}\right\}
$$

\subsubsection{Constraint Solution for 2-DOF Configurations}

The final solution developed is for the 2-DOF wing module configurations. For this mobility, six unique configurations are possible, however referring to Table 4.8 in Section 4.2.2.2, only one combination of constrained branches is possible, represented by the 5-6-7-8 constrained branch configuration, thus four constraint equations are required. The dependent motions for this configuration consist of two platform airfoil rotations in addition to $b_{x}$ and $b_{y}$. For developing the solution, it is

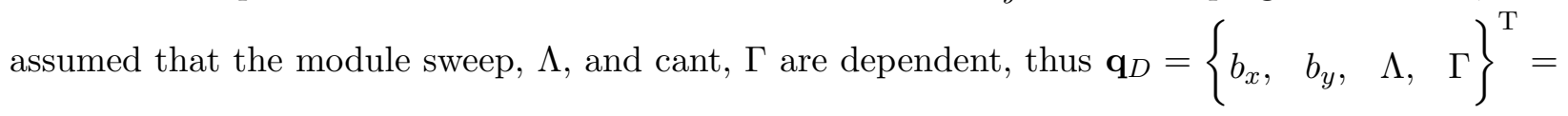
$f\left(b_{z}, \alpha\right)$. The constraint solution for any combination of platform airfoil rotations for the 2-DOF mobility mode is nonlinear due to the presence of the trigonometric terms in Eq. (5.6). However, steps can be taken to reduce the four nonlinear equations by eliminating $b_{x}$ and $b_{y}$ from the system. 
First, terms are grouped, where for each constrained branch

$$
A_{k} \mathrm{c}_{\Lambda}+B_{k} \mathrm{~s}_{\Lambda}+C_{k} \mathrm{c}_{\Lambda} \mathrm{c}_{\Gamma}+D_{k} \mathrm{~s}_{\Lambda} \mathrm{c}_{\Gamma}+E_{k} \mathrm{~s}_{\Lambda} \mathrm{c}_{\Gamma}+F_{k} \mathrm{~s}_{\Lambda} \mathrm{s}_{\Gamma}+G_{k} \mathrm{c}_{\Gamma}+H_{k} \mathrm{~s}_{\Gamma}+n_{k, x} b_{x}+n_{k, y} b_{y}+n_{k, z} b_{z}=0
$$

where

$$
\begin{array}{ll}
A_{k}=n_{k, y}\left(p_{k, x} \mathrm{~s}_{\alpha}+p_{k, y} \mathrm{c}_{\alpha}\right) & E_{k}=-n_{k, y} p_{k, z} \\
B_{k}=n_{k, z}\left(p_{k, x} \mathrm{~s}_{\alpha}+p_{k, y} \mathrm{c}_{\alpha}\right) & F_{k}=n_{k, y}\left(p_{k, x} \mathrm{c}_{\alpha}-p_{k, y} \mathrm{~s}_{\alpha}\right) \\
C_{k}=n_{k, z} p_{k, z} & G_{k}=n_{k, x}\left(p_{k, x} \mathrm{c}_{\alpha}-p_{k, y} \mathrm{~s}_{\alpha}\right) \\
D_{k}=n_{k, z}\left(p_{k, y} \mathrm{~s}_{\alpha}-p_{k, x} \mathrm{c}_{\alpha}\right) & H_{k}=n_{k, x} p_{k, z}
\end{array}
$$

Using a similar system for the 3-DOF constraint solution, one constraint equation is rearranged to isolate for $b_{x}$ and then substituted into the remaining three. For example, using constraint branch 5 to isolate for $b_{x}$ and then substitute into the equation for constraint branch 6 results in

$$
\begin{aligned}
0 & =\left(n_{5, x} A_{6}-n_{6, x} A_{5}\right) \mathrm{c}_{\Lambda}+\left(n_{5, x} B_{6}-n_{6, x} B_{5}\right) \mathrm{s}_{\Lambda}+\left(n_{5, x} C_{6}-n_{6, x} C_{5}\right) \mathrm{c}_{\Lambda} \mathrm{c}_{\Gamma}+\left(n_{5, x} D_{6}-n_{6, x} D_{5}\right) \mathrm{c}_{\Lambda} \mathrm{s}_{\Gamma} \\
& +\left(n_{5, x} E_{6}-n_{6, x} E_{5}\right) \mathrm{s}_{\Lambda} \mathrm{c}_{\Gamma}+\left(n_{5, x} F_{6}-n_{6, x} F_{5}\right) \mathrm{s}_{\Lambda} \mathrm{s}_{\Gamma}+\left(n_{5, x} G_{6}-n_{6, x} G_{5}\right) \mathrm{c}_{\Gamma}+\left(n_{5, x} H_{6}-n_{6, x} H_{5}\right) \mathrm{s}_{\Gamma} \\
& +\left(n_{5, x} n_{6, y}-n_{6, x} n_{5, y}\right) b_{y}+\left(n_{5, x} n_{6, z}-n_{6, x} n_{5, z}\right) b_{z}
\end{aligned}
$$

Similar equations are formed for the constraint equations for branches 7 and 8 . Once again, one equation is used to isolate for $b_{y}$ which is then substituted into the remaining two equations. After performing the substitutions, two constraint equations remain, one with components from branches 5, 6 and 7, and the other with components from branches 5,6 and 8. Using branches 5, 6 and 7 as an example, the constraint equation becomes

$$
\begin{aligned}
0 & =A_{567}^{*} \mathrm{c}_{\Lambda}+B_{567}^{*} \mathrm{~s}_{\Lambda}+C_{567}^{*} \mathrm{c}_{\Lambda} \mathrm{c}_{\Gamma}+D_{567}^{*} \mathrm{c}_{\Lambda} \mathrm{s}_{\Gamma} \\
& +E_{567}^{*} \mathrm{~s}_{\Lambda} \mathrm{c}_{\Gamma}+F_{567}^{*} \mathrm{~s}_{\Lambda} \mathrm{s}_{\Gamma}+G_{567}^{*} \mathrm{c}_{\Gamma}+H_{567}^{*} \mathrm{~s}_{\Gamma}+Z_{567}^{*} b_{z}
\end{aligned}
$$


where

$$
\begin{aligned}
A_{567}^{*} & =\left(\frac{n_{5, x} A_{7}-n_{7, x} A_{5}}{n_{5, x} n_{6, y}-n_{6, x} n_{5, y}}-\frac{n_{5, x} A_{6}-n_{6, x} A_{5}}{n_{5, x} n_{7, y}-n_{7, x} n_{5, y}}\right) \\
B_{567}^{*} & =\left(\frac{n_{5, x} B_{7}-n_{7, x} B_{5}}{n_{5, x} n_{6, y}-n_{6, x} n_{5, y}}-\frac{n_{5, x} B_{6}-n_{6, x} B_{5}}{n_{5, x} n_{7, y}-n_{7, x} n_{5, y}}\right) \\
C_{567}^{*} & =\left(\frac{n_{5, x} C_{7}-n_{7, x} C_{5}}{n_{5, x} n_{6, y}-n_{6, x} n_{5, y}}-\frac{n_{5, x} C_{6}-n_{6, x} C_{5}}{n_{5, x} n_{7, y}-n_{7, x} n_{5, y}}\right) \\
D_{567}^{*} & =\left(\frac{n_{5, x} D_{7}-n_{7, x} D_{5}}{n_{5, x} n_{6, y}-n_{6, x} n_{5, y}}-\frac{n_{5, x} D_{6}-n_{6, x} D_{5}}{n_{5, x} n_{7, y}-n_{7, x} n_{5, y}}\right) \\
E_{567}^{*}= & \left(\frac{n_{5, x} E_{7}-n_{7, x} E_{5}}{n_{5, x} n_{6, y}-n_{6, x} n_{5, y}}-\frac{n_{5, x} E_{6}-n_{6, x} E_{5}}{n_{5, x} n_{7, y}-n_{7, x} n_{5, y}}\right) \\
F_{567}^{*}= & \left(\frac{n_{5, x} F_{7}-n_{7, x} F_{5}}{n_{5, x} n_{6, y}-n_{6, x} n_{5, y}}-\frac{n_{5, x} F_{6}-n_{6, x} F_{5}}{n_{5, x} n_{7, y}-n_{7, x} n_{5, y}}\right) \\
G_{567}^{*}= & \left(\frac{n_{5, x} G_{7}-n_{7, x} G_{5}}{n_{5, x} n_{6, y}-n_{6, x} n_{5, y}}-\frac{n_{5, x} G_{6}-n_{6, x} G_{5}}{n_{5, x} n_{7, y}-n_{7, x} n_{5, y}}\right) \\
H_{567}^{*}= & \left(\frac{n_{5, x} H_{7}-n_{7, x} H_{5}}{n_{5, x} n_{6, y}-n_{6, x} n_{5, y}}-\frac{n_{5, x} H_{6}-n_{6, x} H_{5}}{n_{5, x} n_{7, y}-n_{7, x} n_{5, y}}\right) \\
Z_{567}^{*}= & \left(\frac{n_{5, x} n_{7, z}-n_{7, x} n_{5, z}}{n_{5, x} n_{6, y}-n_{6, x} n_{5, y}}-\frac{n_{5, x} n_{6, z}-n_{6, x} n_{5, z}}{n_{5, x} n_{7, y}-n_{7, x} n_{5, y}}\right)
\end{aligned}
$$

Equation (5.22) is still nonlinear with the module sweep, $\Lambda$, and cant, $\Gamma$ present; the other two dependent variables, $b_{x}$ and $b_{y}$ have been eliminated. Solving Eq. (5.22) can be accomplished using numerical methods, such as Newton-Raphson, and yields the values of $\Lambda$ and $\Gamma$. The remaining dependent motions, $b_{x}$ and $b_{y}$ can now be solved using Eq. (5.9). A similar solution can be obtained using any combination of sweep, cant and twist.

\subsubsection{Instantaneous Kinematics}

The instantaneous kinematics of parallel robots, including the wing module, form a relationship between the platform rate-based variable inputs and the rate-based joint velocity outputs. This relationship, formally known as the system Jacobian, is essentially a transformation between the system global coordinate frame into individual local frames for each controllable joint within the

system. For parallel robot systems, including the wing module, the rate-based relationship takes the form

$$
\mathbf{J}_{f w d} \dot{\mathbf{d}}=\mathbf{J}_{i n v} \mathbf{t}_{p}
$$




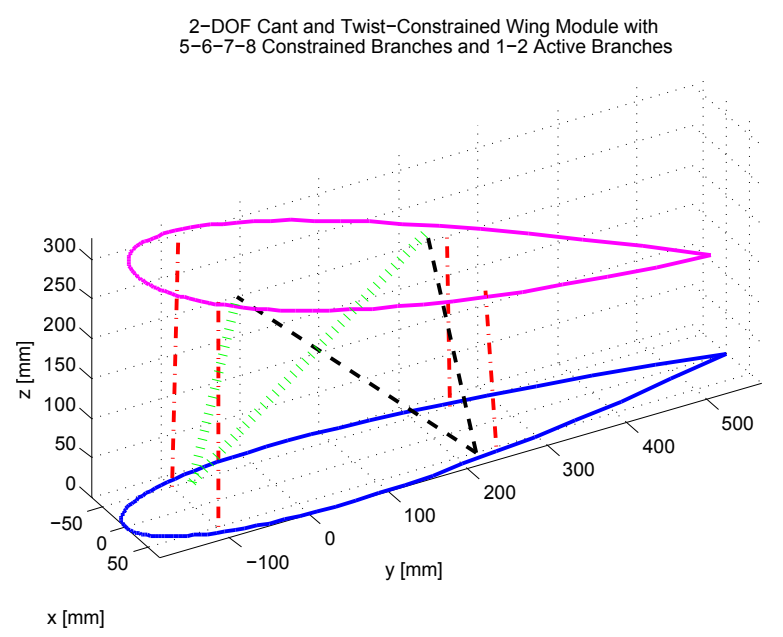

(a) Active Sweep with $\Lambda=-15^{\circ}$

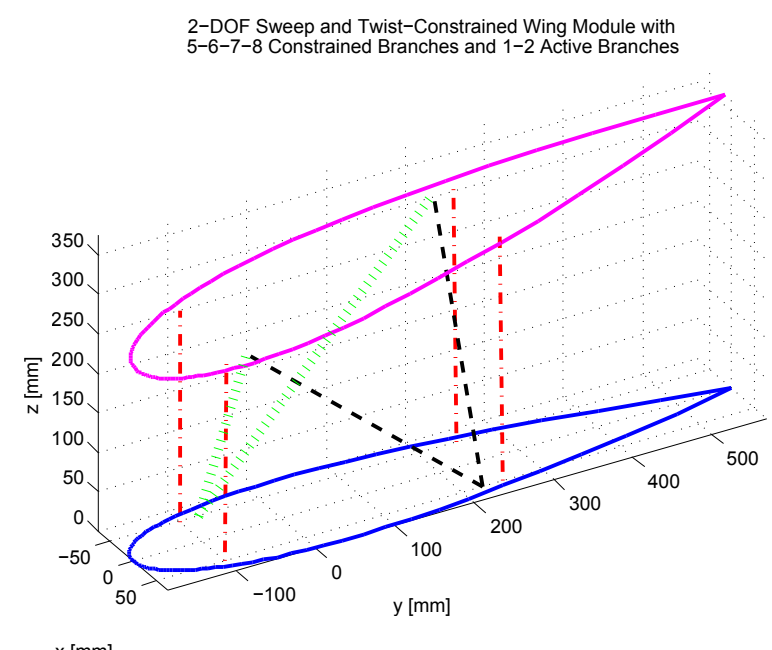

(b) Active Cant with $\Gamma=10^{\circ}$

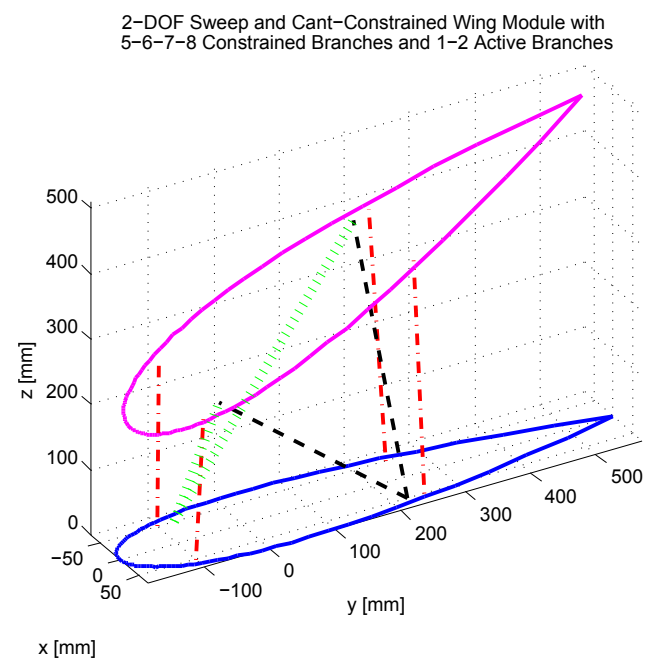

(c) Active Twist with $\alpha=-1^{o}$

Figure 5.5: 2-DOF wing modules with red constrained branches and $b_{z}=275 \mathrm{~mm}$

where $\dot{\mathbf{d}}$ is the vector of joint velocities, $\mathbf{t}_{p}$ is the instantaneous twist of the platform airfoil, and $\mathbf{J}_{f w d}$ and $\mathbf{J}_{i n v}$ are the forward and inverse Jacobian matrices. The platform instantaneous twist vector is a

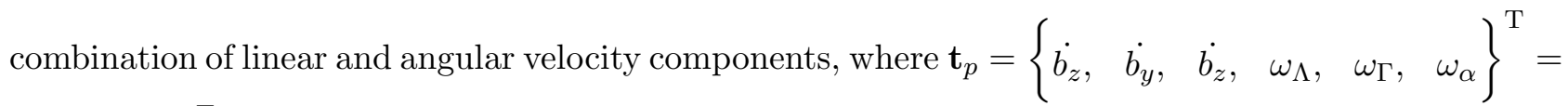
$\left\{\dot{\mathbf{h}}^{\mathrm{T}}, \quad \boldsymbol{\omega}^{\mathrm{T}}\right\}^{\mathrm{T}}$. The platform airfoil twist vector is not to be confused with wing twist variable associated with the mobility of the module, as the twist vector is derived from screw theory.

To form the system Jacobian, the derivative of the branch vector loop, Eq (5.1), is taken

$$
\dot{\mathbf{d}}_{k}+\boldsymbol{\omega}_{\mathbf{d}_{k}} \times \mathbf{d}_{k}=\dot{\mathbf{b}}+\boldsymbol{\omega} \times \mathbf{p}_{p, k}
$$


where for branch $k, \dot{\mathbf{d}}_{k}$ and $\boldsymbol{\omega}_{\mathbf{d}_{k}}$ are the linear and angular velocity components, respectively and where for the platform airfoil, $\dot{\mathbf{b}}$ and $\boldsymbol{\omega}$ are the linear and angular velocity components, respectively. The base airfoil vectors do not contribute to the velocity as the vectors are stationary within the module frame. Equation (5.25) is the velocity vector loop and holds for each branch within the system, thus forming the aforementioned rate-based relationship. Each side of Eq. (5.25) calculates the spherical joint velocity with respect to the module base airfoil.

In Eq. (5.25), six velocity components for the branch are present, however only the controllable joint velocity is needed. As the active branches of the wing module are of UPS architecture, with an active prismatic joint, the only velocity component needed is that which aligns with the prismatic actuator. In this case, this velocity is the summation of linear velocity components that align with $\mathbf{d}_{k}$. All other velocity components, including the angular velocity components of the branch, are orthogonal and can be eliminated by projecting Eq. (5.25) onto $\mathbf{d}_{k}$, which after elimination becomes

$$
\dot{d}_{k}=\dot{\mathbf{b}} \cdot \mathbf{d}_{k}+\boldsymbol{\omega} \times \mathbf{p}_{p, k} \cdot \mathbf{d}_{k}
$$

Equation (5.26) can be rearranged such that the instantaneous twist of the platform can be extracted. Doing so yields

$$
\dot{d}_{k}=\left\{\mathbf{d}_{k}^{\mathrm{T}}, \quad\left(\mathbf{p}_{p, k} \times \mathbf{d}_{k}\right)^{\mathrm{T}}\right\} \mathbf{t}_{p}
$$

Finally, Eq. (5.27) is formed for each active branch within the wing module system and combined to form the system of linear equations in the form of Eq. (5.24). In general, the Jacobian system for a parallel robot with mobility $M$ takes the form

$$
\left[\begin{array}{cccc}
1 & 0 & \cdots & 0 \\
0 & 1 & \ddots & \vdots \\
\vdots & \ddots & \ddots & 0 \\
0 & \cdots & 0 & 1
\end{array}\right]\left\{\begin{array}{c}
\dot{d}_{1} \\
\dot{d}_{2} \\
\vdots \\
\dot{d}_{M}
\end{array}\right\}=\left[\begin{array}{cc}
\mathbf{d}_{1}^{\mathrm{T}} & \left(\mathbf{p}_{p, 1} \times \mathbf{d}_{1}\right)^{\mathrm{T}} \\
\mathbf{d}_{2}^{\mathrm{T}} & \left(\mathbf{p}_{p, 2} \times \mathbf{d}_{2}\right)^{\mathrm{T}} \\
\vdots & \vdots \\
\mathbf{d}_{1}^{\mathrm{T}} & \left(\mathbf{p}_{p, 1} \times \mathbf{d}_{1}\right)^{\mathrm{T}}
\end{array}\right]\left\{\begin{array}{c}
\dot{\mathbf{b}} \\
\boldsymbol{\omega}
\end{array}\right\}
$$

We can form the Jacobian as

$$
\mathbf{J}=\mathbf{J}_{f w d}^{-1} \mathbf{J}_{i n v}
$$


where we see that $\mathbf{J}=\mathbf{J}_{i n v}$, as the inverse of the identity forward Jacobian is also the identity matrix.

\subsubsection{Parametric Velocity Constraint}

For the limited mobility modes the wing module assumes, the Jacobian matrix of Eq. (5.28) is of size $M \times 6$, and as such is not directly invertible. If the platform instantaneous twist is known in full, then via the Jacobian transformation, the joint velocities can be determined. Conversely, if joint velocities are known, then the platform instantaneous twist can be determined via the inverse Jacobian. Since for the wing module the Jacobian matrix is never square, the pseudo-inverse must be used when calculating the platform twist for a given set of joint velocities. Alternatively constrained Jacobian matrices have been proposed that are square, and thus are directly invertible $[70,71]$.

The platform twist vector $\mathbf{t}$ contains a mix of both independent and dependent platform velocity components, regardless of the wing module mobility mode as $\dot{b}_{x}$ and $\dot{b}_{y}$ are always dependent velocities. From Eq.(5.4), the spherical joint within the branch is required to move within the constraint plane and the resultant velocity of the spherical joint must also be on this plane. Thus the derivative of Eq. (5.4) can be used to form a relationship between the dependent and independent platform velocities. After taking the time derivative of Eq. (5.4), the velocity constraint becomes

$$
\left(\dot{\mathbf{b}}+\boldsymbol{\omega} \times \mathbf{p}_{p, k}\right) \cdot \mathbf{n}_{k}=0
$$

which is the parametric velocity constraint. Once again for simplicity, the platform airfoil vectors are expressed as $\mathbf{p}_{k}$ as only the platform airfoil vector appears in Eq. (5.30). Expanding Eq. (5.30) and collecting platform instantaneous twist components results in

$$
\left\{n_{k, x}, \quad n_{k, y}, \quad n_{k, z}, \quad\left(n_{k, y} p_{k, z}-n_{k, z} p_{k, y}\right), \quad\left(n_{k, z} p_{k, x}-n_{k, x} p_{k, z}\right), \quad\left(n_{k, x} p_{k, y}-n_{k, y} p_{k, x}\right)\right\} \mathbf{t}_{p}=0
$$

In this form, Eq. (5.31) can be used to form the specific system of equations in which the dependent velocity components are related to the independent velocity components. Symbolically, 
this relationship is in the form

$$
\mathbf{J}_{\text {dep }} \mathbf{t}_{d e p}=\mathbf{J}_{\text {ind }} \mathbf{t}_{\text {ind }}
$$

where the independent Jacobian, $\mathbf{J}_{\text {ind }}$, and dependent Jacobian $\mathbf{J}_{\text {dep }}$ relate the independent and dependent twist vectors, $\mathbf{t}_{\text {ind }}$ and $\mathbf{t}_{d e p}$, respectively. Within the system there are an equal number of velocity constraints as there are platform constraints, thus $\mathbf{J}_{d e p}$ is square of size $M \times M$. Therefore it can be inverted such that

$$
\mathbf{t}_{d e p}=\mathbf{J}_{d e p}^{-1} \mathbf{J}_{\text {ind }} \mathbf{t}_{\text {ind }}=\mathbf{J}_{\mathbf{t}} \mathbf{t}_{\text {ind }}
$$

where $\mathbf{J}_{\mathbf{t}}$ is the twist Jacobian. Finally, the rows of Eq. (5.33) are aligned accordingly such that $\mathbf{t}_{p}=\left\{\mathbf{t}_{\text {ind }}, \quad \mathbf{t}_{d e p}\right\}^{\mathrm{T}}=\left\{\mathbf{t}_{\text {ind }}, \quad \mathbf{J}_{\mathbf{t}} \mathbf{t}_{\text {ind }}\right\}^{\mathrm{T}}$ and

$$
\dot{\mathbf{d}}=\mathbf{J J}_{\mathbf{t}} \mathbf{t}_{\text {ind }}
$$

where the constrained Jacobian $\mathbf{J}_{c}$ is defined as

$$
\mathbf{J}_{c}=\mathbf{J J}_{\mathbf{t}}
$$

This is the $M \times M$ matrix that represents the transformation between independent platform twist components to the velocities of the unconstrained-active prismatic joint velocities.

\subsection{Kinematic Evaluation}

In this section, the kinematics of the wing module are evaluated with respect to various performance parameters. The goal is to not only show how the module will respond to kinematic input, but also to contrast the performance of each configuration for each mobility mode. Ultimately, module selection is made based not only on the mobility requirement of the module, but also on which configuration provides the best kinematic performance.

For the simulations, the base and platform airfoils each have a chord length of $750 \mathrm{~mm}$, with the coordinate frames centered at the $1 / 4$-chord points. Connection points for the the branch vectors are summarized in Table 5.1. Also listed are the stroke limits for each branch which correspond to the total physical length of each branch of the developed prototype (branches 1 and 3 have 
Table 5.1: Wing module branch parameters

\begin{tabular}{|c|c|c|c|c|}
\hline \multirow{3}{*}{$\begin{array}{l}\text { Branch } \\
1\end{array}$} & \multirow{3}{*}{ 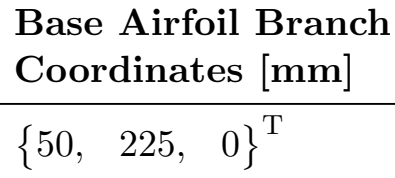 } & \multirow{3}{*}{$\begin{array}{l}\text { Platform Airfoil Branch } \\
\text { Coordinates [mm] }\end{array}$} & \multicolumn{2}{|c|}{ Stroke Length [mm] } \\
\hline & & & Minimum & Maximum \\
\hline & & & 283.7 & 421.7 \\
\hline 2 & $\{50, \quad 225, \quad 0\}^{\mathrm{T}}$ & $\{50,225, \quad 0\}^{\mathrm{T}}$ & 207.5 & 345.5 \\
\hline 3 & $\left\{\begin{array}{lll}-50, & -75, & 0\end{array}\right\}^{\mathrm{T}}$ & $\left\{\begin{array}{lll}-50, & 225, & 0\end{array}\right\}^{\mathrm{T}}$ & 283.7 & 421.7 \\
\hline 4 & $\left\{\begin{array}{lll}-50, & -75, & 0\end{array}\right\}^{\mathrm{T}}$ & $\{50,-75, \quad 0\}^{\mathrm{T}}$ & 207.5 & 345.5 \\
\hline 5 & $\left\{\begin{array}{lll}50, & 250, & 0\end{array}\right\}^{\mathrm{T}}$ & $\{50,250, \quad 0\}^{\mathrm{T}}$ & 207.5 & 345.5 \\
\hline 6 & $\left\{\begin{array}{lll}-50, & 250, & 0\end{array}\right\}^{\mathrm{T}}$ & $\left\{\begin{array}{lll}-50, & 250, & 0\end{array}\right\}^{\mathrm{T}}$ & 207.5 & 345.5 \\
\hline 7 & $\left\{\begin{array}{lll}-50, & -100, & 0\end{array}\right\}^{\mathrm{T}}$ & $\left\{\begin{array}{lll}-50, & -100, & 0\end{array}\right\}^{\mathrm{T}}$ & 207.5 & 345.5 \\
\hline 8 & $\left\{\begin{array}{lll}50, & -100, & 0\end{array}\right\}^{\mathrm{T}}$ & $\left\{\begin{array}{lll}50, & -100, & 0\end{array}\right\}^{\mathrm{T}}$ & 207.5 & 345.5 \\
\hline
\end{tabular}

added extensions to account for the additional requied length of the axial branches; see Chapter 6 for figures). The operational limit of the spherical joint at the platform airfoil is restricted to be within $30^{\circ}$ of the home configuration. Here, we define the home configuration of the module to be $\mathbf{b}_{\mathrm{h}}=\{0,0,250\}^{\mathrm{T}}, \mathrm{mm}$, and no platform rotation. At this configuration, the direction of each branch is simply $\hat{\mathbf{d}}_{k, \mathrm{~h}}=\left|\mathbf{b}_{\mathrm{h}}+\mathbf{p}_{k, p}^{\prime}-\mathbf{p}_{k, b}\right| /\left|\mathbf{d}_{k, \mathrm{~h}}\right|$. Thus we can see that the operational limit of each spherical joint is $\arccos \left(R * \hat{\mathbf{d}}_{k, \mathrm{~h}} \cdot \hat{\mathbf{d}}_{k}\right) \leq \pi / 6$. Lastly, the constraint planes are oriented perpendicular to the base airfoil, and pass through the base airfoil coordinate frame $z$-axis.

\subsubsection{Module Kinematic Range}

The kinematic performance of the wing module depends on the operational range of the controllable airfoil degrees-of-freedom. In this section, the variable range for the wing module configurations listed in Table 4.8 are evaluated for each mobility. The sweep, cant and twist for each configuration is dependent on the module span. This is due to the operational limits of the prismatic joints within the system, as well as the operational limits of the spherical joints. Minimum and maximum platform sweep, cant and twist values are listed in the subsequent tables The figures with the 
Table 5.2: Kinematic range for the 4-DOF wing module configurations at $b_{z}=250 \mathrm{~mm}$

\begin{tabular}{lllllll}
\hline $\begin{array}{l}\text { Constrained } \\
\text { Branches }\end{array}$ & \multicolumn{2}{l}{ Platform } & \multicolumn{2}{c}{ Airfoil Variable Range $[\mathbf{d e g}]$} & \multicolumn{2}{c}{} \\
\hline $5-6$ & $\boldsymbol{\Lambda}_{\max }$ & $\boldsymbol{\Lambda}_{\min }$ & $\boldsymbol{\Gamma}_{\max }$ & $\boldsymbol{\Gamma}_{\min }$ & $\boldsymbol{\alpha}_{\max }$ & $\boldsymbol{\alpha}_{\min }$ \\
$5-7$ & 18.0 & -14.0 & 29.0 & -28.0 & 37.5 & -27.5 \\
$5-8$ & 20.5 & -12.0 & 30.0 & -28.0 & 3.5 & -4.0 \\
$6-7$ & 21.0 & -12 & 30.0 & -27.0 & 8.0 & -8.5 \\
$6-8$ & 21.0 & -13.5 & 27.0 & -30.0 & 9.0 & -7.5 \\
$7-8$ & 21.0 & -12.0 & 30.0 & -31.5 & 5.0 & -3.5 \\
\hline
\end{tabular}

following sections depict the distribution of these variables.

\subsubsection{4-DOF Module Kinematic Range}

The 4-DOF wing module consists of four active branches and two constrained branches, leading to six possible configurations. A visual representation of the joint variable range is difficult for the 4-DOF wing modules as the four degrees-of-freedom, if plotted, represent a manifold in 4-space. As such, a midpoint span of $b_{z}=250 \mathrm{~mm}$ is used to evaluate the platform angular ranges, in lieu of using the full span range.

Table 5.2 presents the range of platform airfoil variables at a span of $b_{z}=250 \mathrm{~mm}$. The physical interpretation of the constraint system here is finding the set of lines between the two constraint planes which spans the distance between the spherical joints on the constrained branches. Here we see that the range of platform sweep and cant is fairly consistent between the different constraint branch combinations. Thus we can conclude that the sweep and cant variation is fairly independent of the combination constrained branches. This is because as the module varies the sweep and cant, the overall motion of platform airfoil tends to be in the direction in which the constrained branch spherical joints are forced to move. This alignment of motions allows for an increased range in platform motions for the sweep and cant variables. The limiting factor for these motions is therefore dominated by the operational limits of the prismatic and spherical joints. 


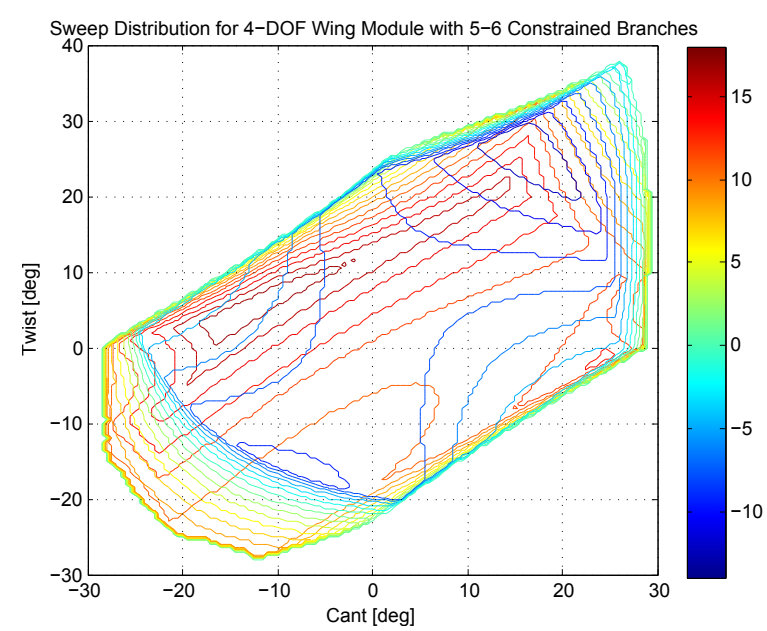

(a) 5-6 Constrained Branches

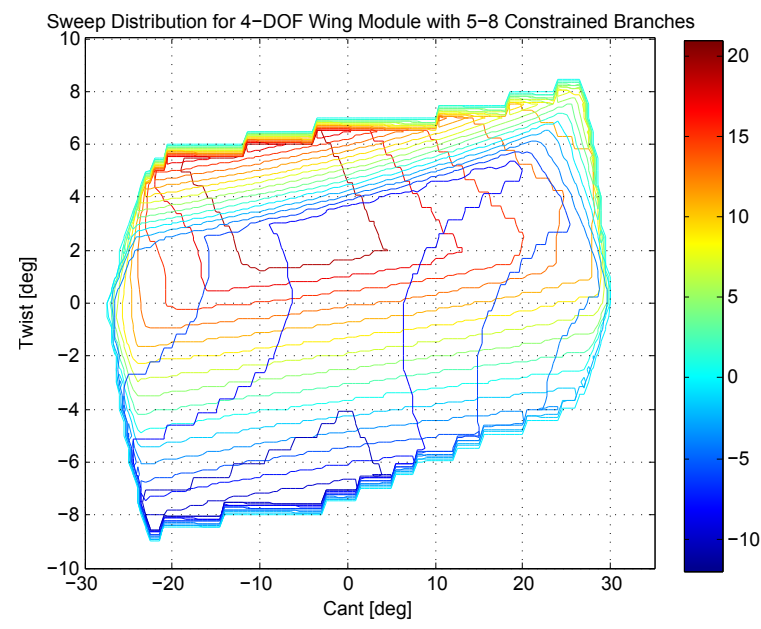

(c) 5-8 Constrained Branches

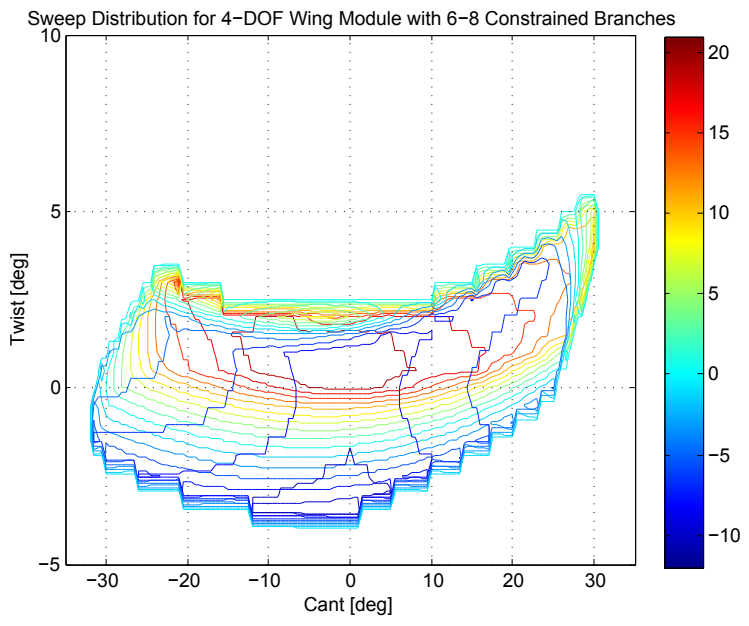

(e) 6-8 Constrained Branches

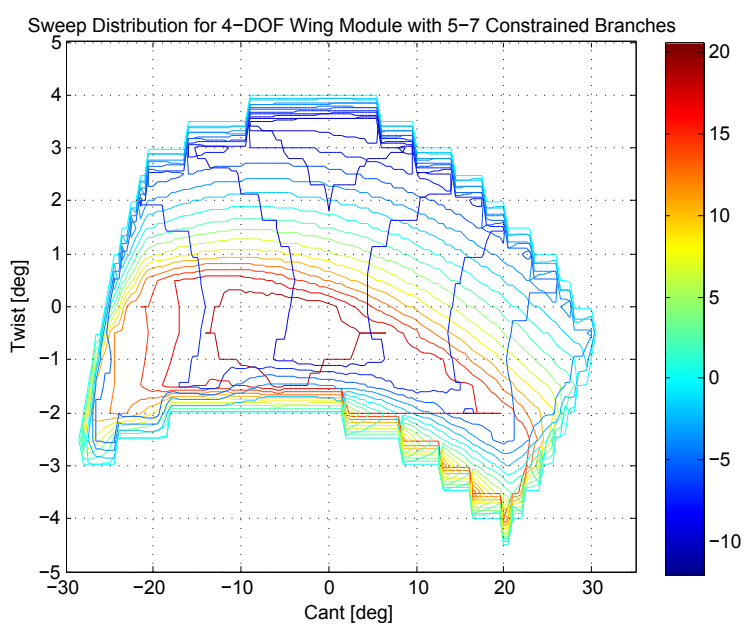

(b) 5-7 Constrained Branches

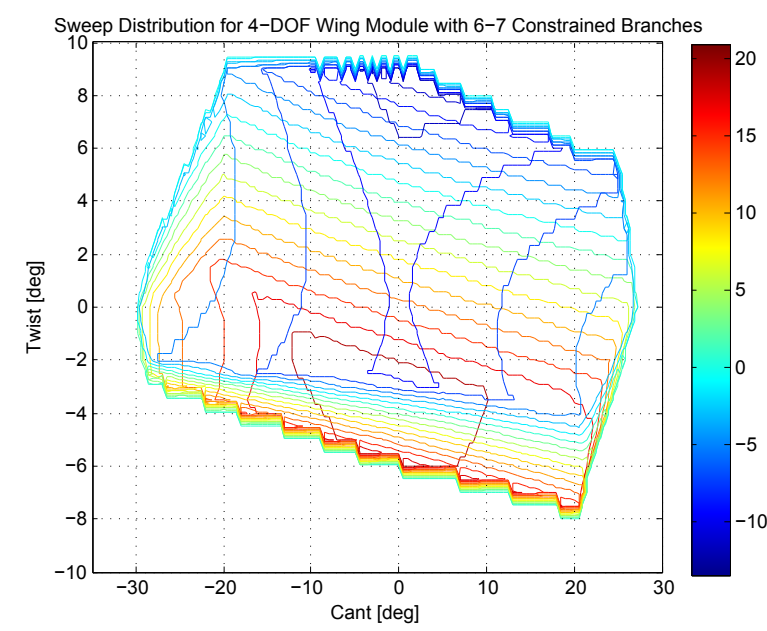

(d) 6-7 Constrained Branches

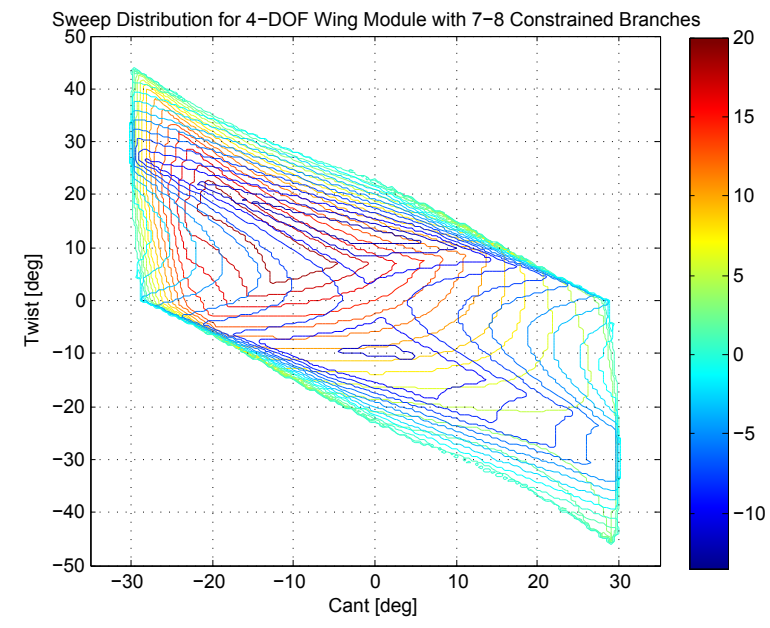

(f) 7-8 Constrained Branches

Figure 5.6: Sweep range of 4-DOF wing modules at $b_{z}=250 \mathrm{~mm}$ 
This is not the case, however with the platform twist, $\alpha$ as there are very large differences between configurations. For twist variation, the range is predominantly dependent on the constraint planes. First we see that the 5-7 and 6-8 configurations allow for the smallest amount of twist, bound by approximately $\pm 5^{\circ}$. These configurations represent constrained branches on opposite quadrants of the base airfoil coordinate frame (refer to Table 5.1) for constrained branch locations. These configurations have constraint planes separated by approximately $15^{\circ}$. The smaller the angular spacing between constraint planes, the closer the configuration is to becoming degenerate in which constrained platform motion becomes increasingly more difficult to achieve. If the two constraint planes are co-planar, then the configuration is degenerate as the system loses the ability to achieve suitable motions. This effect was explored with a symmetric parallel robot in $[59,64]$.

Next, the 5-8 and 6-7 configurations have platform twist bound by approximately $\pm 10^{\circ}$. These configurations represent the two constrained branches being located in the upper or lower regions of the wing module, respectively, with one branch located in the trailing edge region, and the other in the leading edge region. The increased range in platform twist is associated with fact that the constraint planes are separated by approximately $38^{\circ}$. Despite the larger range, the increase is modest compared to the twist range of the 5-6 and 7-8 configurations. These configurations represent both constrained branches located at trailing edge region, or leading edge region, respectively. For the 5-6 configuration, the constraint planes are separated by approximately $23^{\circ}$, but the increase in twist range is associated with a larger set of lines between the constraint planes, that span the distance between the constrained branch spherical joints. The 7-8 configuration on the other hand benefits from the constraint planes being separated by approximately $53^{\circ}$. A correlation becomes apparent between the angular spacing of constraint planes and the set of lines between the two planes that span the distance between the constrained branch spherical joints. The 5-6 and 7-8 configurations possess these qualities and thus allow very large twist range for the airfoil platform.

Not only are the variable ranges important, but also how the platform variable limits effect the others. To explore this, overlapping contour plots of platform sweep (Fig. 5.6), cant (Fig. 5.7), and twist (Fig. 5.8) show minimum and maximum variable ranges with respect to the other variable 
values. For each figure, the colourbar represents the variation in the particular platform variable. For instance, for Fig. 5.6, the sweep variation is shown with respect to the module cant and twist. The coloured contours and colourbar are representative of the minimum and maximum module sweep with respect to the module cant and twist. Likewise, for Fig. 5.7, the coloured contours and colourbar are representative of the minimum and maximum module cant with respect to the module sweep and twist. The same coloured contour representation is used for the twist variation in Fig. 5.8.

For the sweep variation, we see that maximum values occur when the platform cant and twist are close to $0^{\circ}$. Figure 5.6(b), Fig. 5.6(c), Fig. 5.6(d), and Fig. 5.6(e) show the 5-7, 5-8, 6-7, and 6-8 configurations respectively, and from the contours, positive and negative platform sweep is predominantly sided with either positive or negative twist, whereas this is not the case with respect to cant variation. The 5-6 and 7-8 configurations, Fig. 5.6(a) and Fig. 5.6(f) on the other hand show combined cant and twist correlation between extreme sweep motion. The shape of the workspace is almost mirrored between the two configurations. For the 5-6 configuration, the sweep workspace is predominantly within the range of positive cant and twist, or negative cant and twist. The 7-8 configuration on the other hand has the sweep workspace is predominant when the cant twist are of opposite sign.

Cant variation of the platform is predominantly dependent on sweep for the 5-6 and 7-8 configurations, Fig. 5.7(a) and Fig. 5.7(f), respectively. Here, maximum and minimum cant values occur at opposite signs of twist. For the 5-6 configuration, maximum and minimum cant occur with positive and negative twist, and vice versa for the 7-8 configuration. The remaining configurations, 5-7, 5-8, 6-7, and 6-8, Fig. 5.7(b), Fig. 5.7(c), Fig. 5.7(d), and Fig. 5.7(e), respectively each show that there is little variation in cant ranges with respect to the platform twist. The exception here is the maximum cant for the 5-7 configuration occurs at a negative twist. For all configurations, sweep variation, except at extreme values, has little effect on cant variation.

Finally, the maximum and minimum twist values dependent on both the cant and sweep. Using the same groupings as analyzed above, we see trends for the 5-7 and 6-8, 5-8 and 6-7, and 5-6 


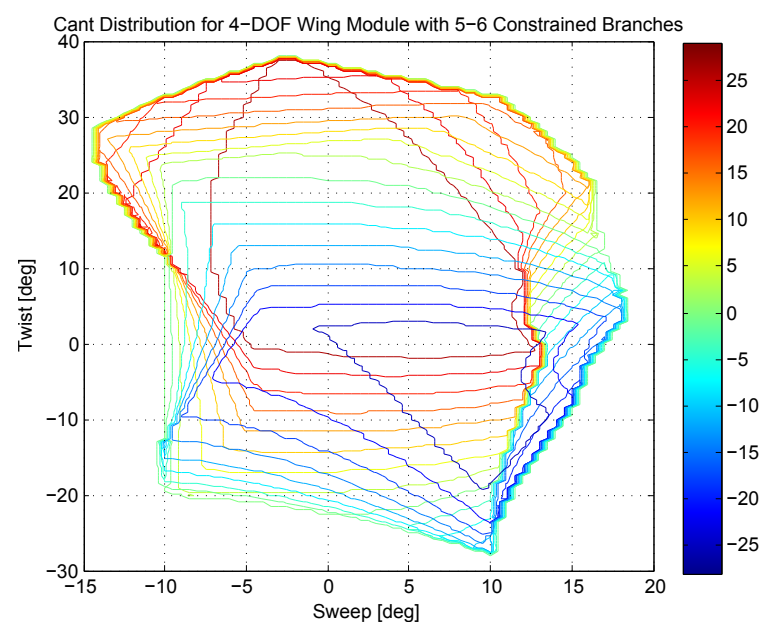

(a) 5-6 Constrained Branches

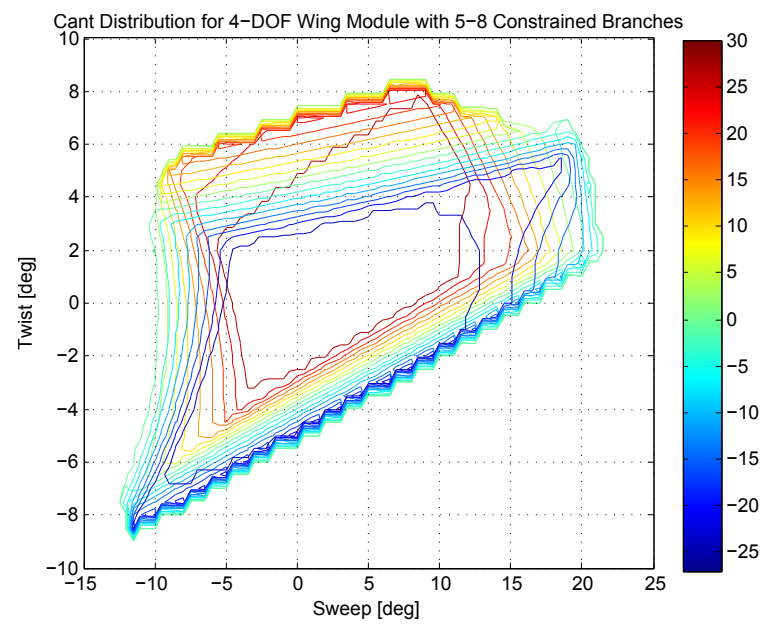

(c) 5-8 Constrained Branches

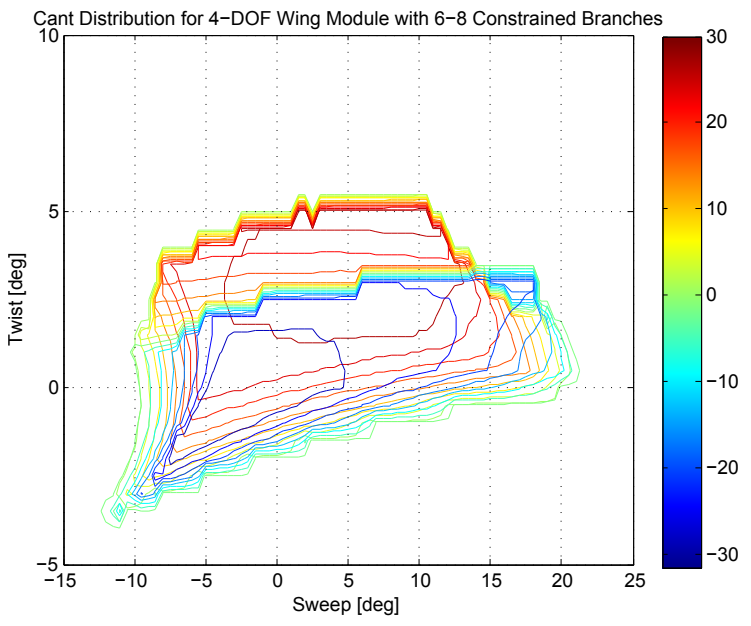

(e) 6-8 Constrained Branches

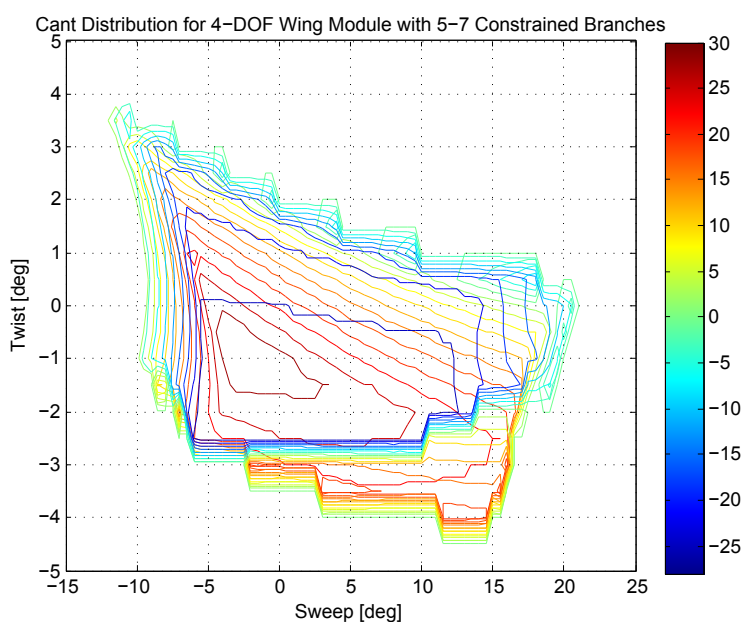

(b) 5-7 Constrained Branches

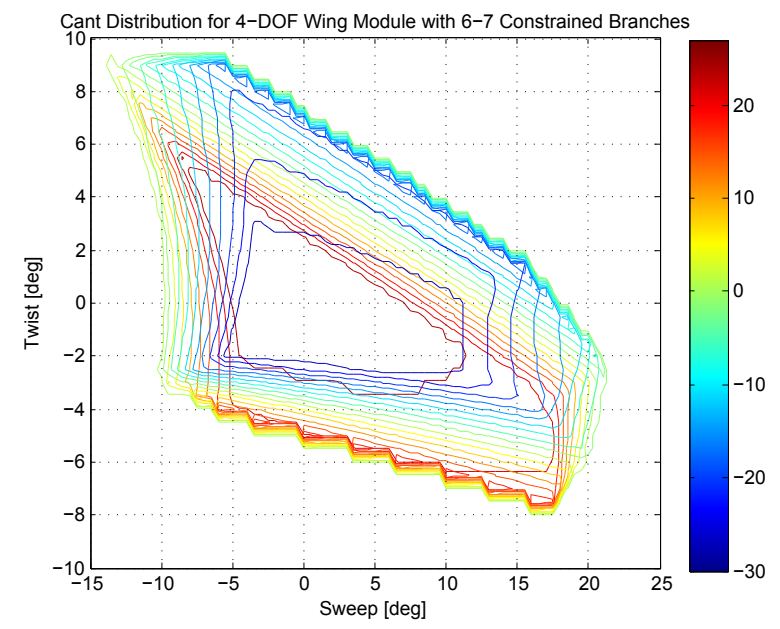

(d) 6-7 Constrained Branches

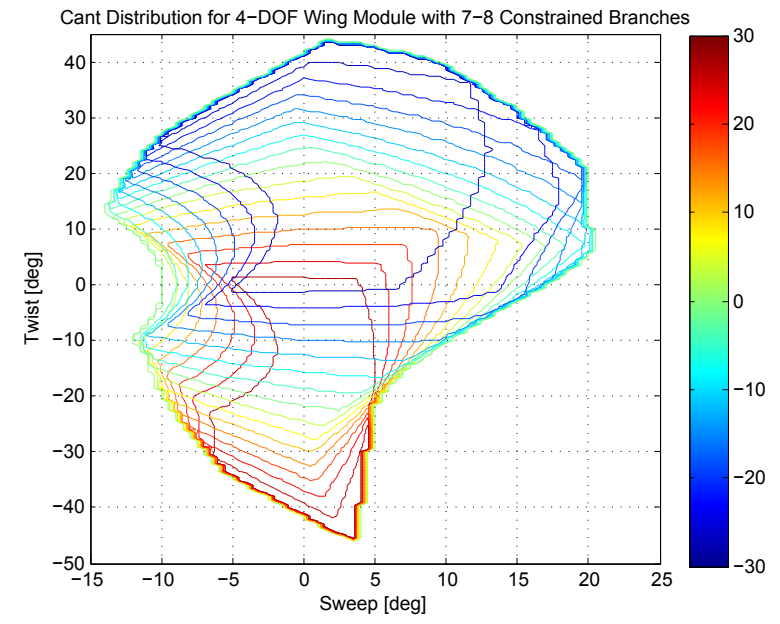

(f) 7-8 Constrained Branches

Figure 5.7: Cant range of 4-DOF wing modules at $b_{z}=250 \mathrm{~mm}$ 


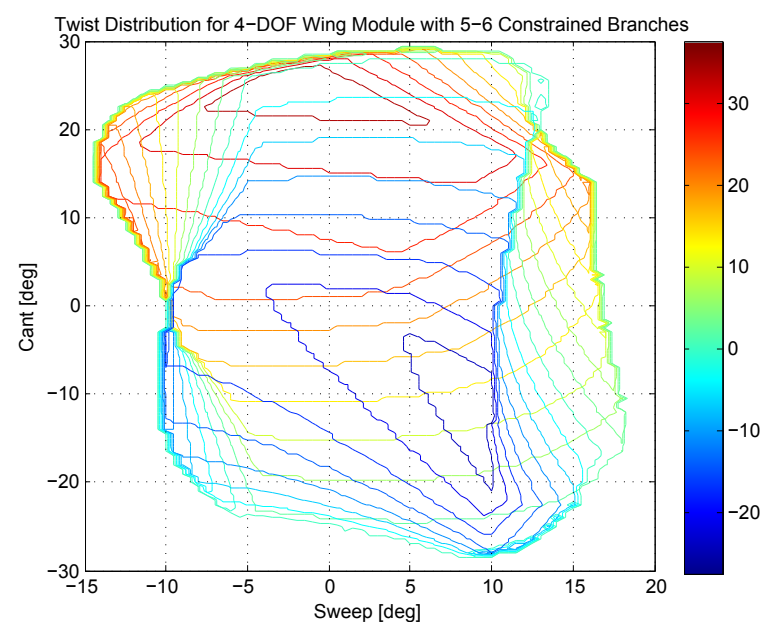

(a) 5-6 Constrained Branches

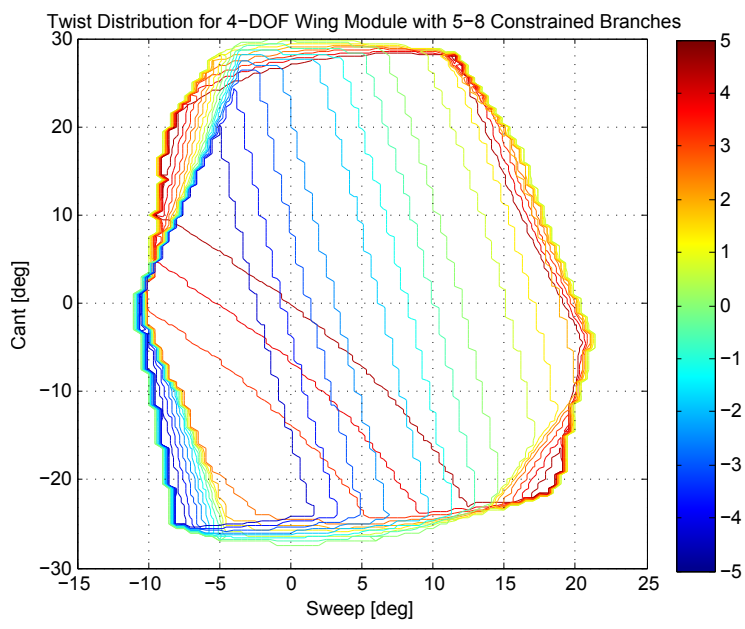

(c) 5-8 Constrained Branches

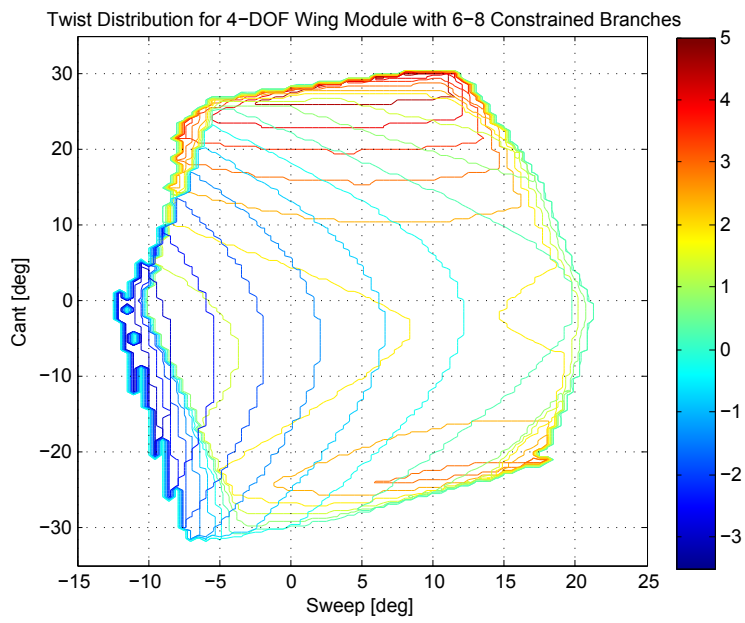

(e) 6-8 Constrained Branches

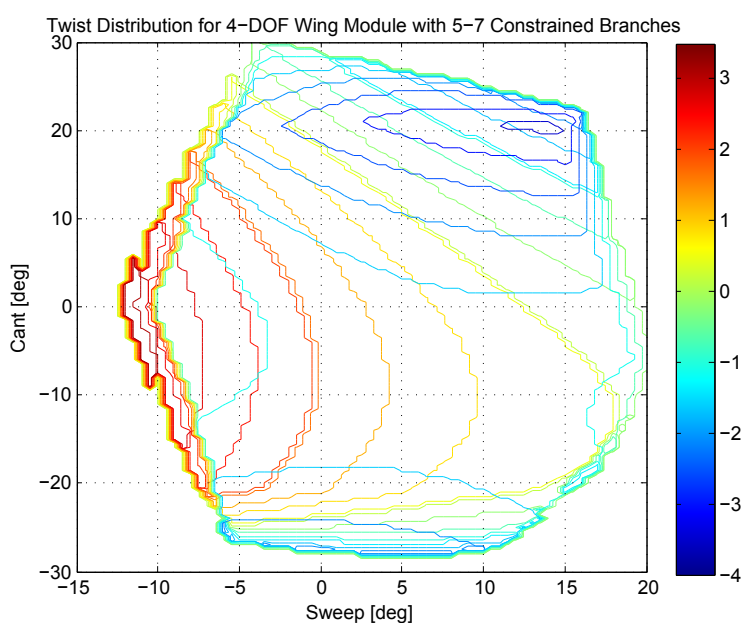

(b) 5-7 Constrained Branches

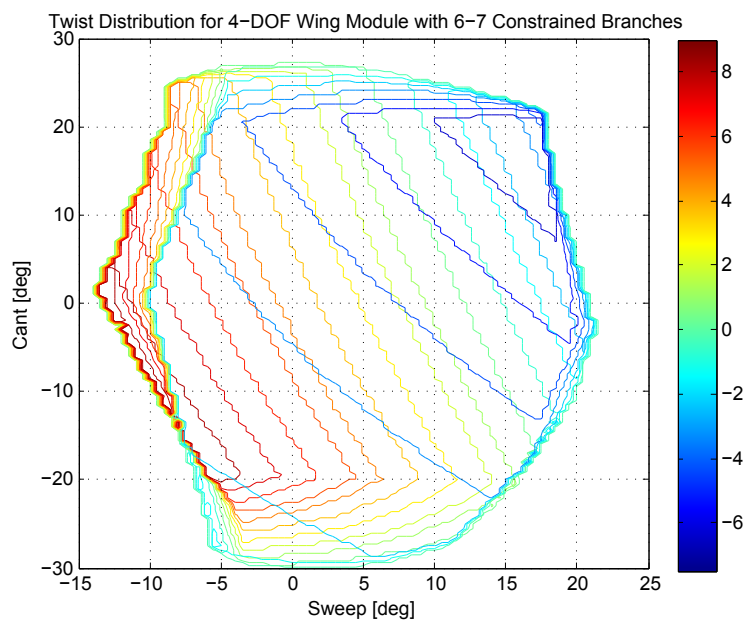

(d) 6-7 Constrained Branches

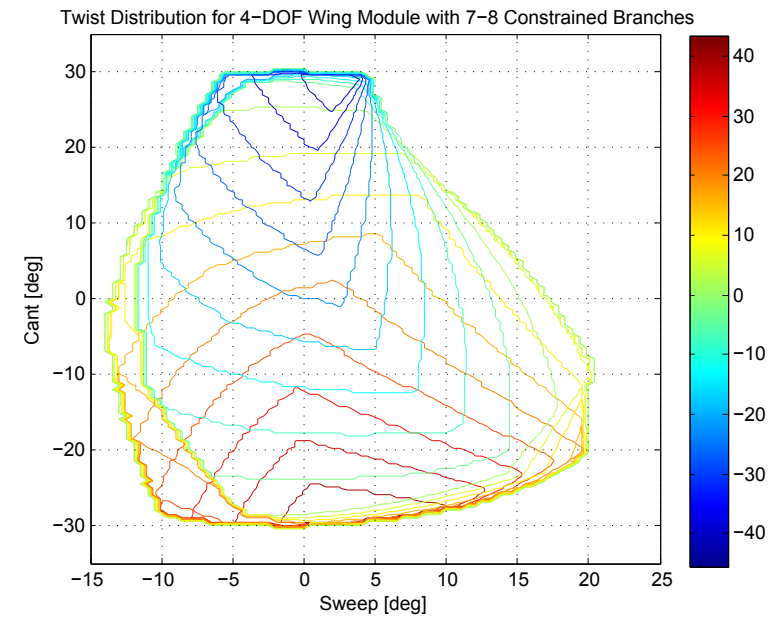

(f) 7-8 Constrained Branches

Figure 5.8: Twist range of 4 -DOF wing modules at $b_{z}=250 \mathrm{~mm}$ 
and 7-8 configurations. Starting with the 5-7 and 6-8 configurations, Fig. 5.8(b) and Fig. 5.8(e), respectively, have opposite twist distributions. For the 5-7 configuration, a maximum twist occurs at negative sweep and small values of cant. The minimum twist occurs at a positive cant and sweep. The 6-8 configuration is opposite with maximum twist occurring with a positive cant and sweep, and minimum twist occurring at a negative sweep and small values of cant. Next, the 5-8 and 6-7 configurations, Fig. 5.8(c) and Fig. 5.8(d), respectively, also have opposite twist distributions. The 5-8 configuration has minimum and maximum twist in the negative cant and sweep, and positive cant and sweep regions, respectively. The minimum and maximum values are switched for the 6-7 configuration. Lastly, the 5-6 and 7-8 configurations, Fig. 5.8(a) and Fig. 5.8(f), respectively, represent the configurations with the largest twist variation. These configurations exhibit less of a dependency on sweep variation when observing the twist distribution, as the minimum and maximum twist values are predominantly determined by the module cant.

The 4-DOF wing modules represent the configurations with the largest variable reach. The uneven distribution of branches about the base coordinate frame does not lead to isomorphic configurations, however strong kinematic correlations develop between configurations with similar placement of passive branches. While sweep and cant range is relatively consistent between the configurations, the twist range is not. The 5-7 and 6-8 configurations represent diagonally opposite constrained branches, which severely limit the twist range as described above. The 5-8 and 6-7 configurations have constrained branches either within the upper or lower region of the module, respectively, and although these configurations expands the twist range versus the 5-7 and 6-8 configurations, the twist range is still limited with respect to that of the 5-6 and 7-8 configurations. The 5-6 and 7-8 configurations have constrained branches in the trailing and leading edge regions, respectively.

\subsubsection{3-DOF Module Kinematic Range}

The 3-DOF wing module consists of four constrained branch configurations, each capable of providing two independent rotations to the platform. The remaining rotational motion is dependent. 
Table 5.3: Kinematic range for the 3-DOF wing module configurations

\begin{tabular}{|c|c|c|c|c|c|c|c|c|c|}
\hline \multirow{2}{*}{$\begin{array}{l}\text { Const. } \\
\text { Branches }\end{array}$} & \multirow{2}{*}{$\begin{array}{l}\text { Dep. } \\
\text { Mot. }\end{array}$} & \multicolumn{2}{|c|}{ Range [deg] } & \multirow{2}{*}{$\begin{array}{l}\text { Dep. } \\
\text { Mot. }\end{array}$} & \multicolumn{2}{|c|}{ Range [deg] } & \multirow{2}{*}{$\begin{array}{l}\text { Dep. } \\
\text { Mot. }\end{array}$} & \multicolumn{2}{|c|}{ Range [deg] } \\
\hline & & $\Lambda_{\max }$ & $\boldsymbol{\Lambda}_{\min }$ & & $\boldsymbol{\Gamma}_{\max }$ & $\boldsymbol{\Gamma}_{\min }$ & & $\boldsymbol{\alpha}_{\max }$ & $\boldsymbol{\alpha}_{\min }$ \\
\hline \multirow[t]{2}{*}{$5-6-7$} & $\Gamma$ & 14.5 & -18.0 & $\Lambda$ & 25.0 & -28.5 & $\Lambda$ & 1.25 & -3.25 \\
\hline & $\alpha$ & 17.0 & -19.0 & $\alpha$ & 26.0 & -28.5 & $\Gamma$ & 1.25 & -3.25 \\
\hline \multirow[t]{2}{*}{$5-6-8$} & $\Gamma$ & 17.5 & -18.0 & $\Lambda$ & 28.5 & -24.5 & $\Lambda$ & 2.5 & -1.25 \\
\hline & $\alpha$ & 17.5 & -18.5 & $\alpha$ & 28.5 & -26.5 & $\Gamma$ & 3.5 & -1.25 \\
\hline \multirow[t]{2}{*}{$5-7-8$} & $\Gamma$ & 18.0 & -14.5 & $\Lambda$ & 28.5 & 26.5 & $\Lambda$ & 0.5 & -1.25 \\
\hline & $\alpha$ & 18.5 & -16.0 & $\alpha$ & 28.5 & -27.0 & $\Gamma$ & 0.5 & -1.25 \\
\hline \multirow[t]{2}{*}{$6-7-8$} & $\Gamma$ & 18.0 & -16.0 & $\Lambda$ & 26.5 & -28.5 & $\Lambda$ & 1.5 & -0.5 \\
\hline & $\alpha$ & 18.5 & -16.0 & $\alpha$ & 27.0 & -28.5 & $\Gamma$ & 1.5 & -0.5 \\
\hline
\end{tabular}

Thus a total of twelve combinations of branch constraints and platform motions are possible. For this case, the span-wise distribution of variables can be visualized due to the presence of three independent motions.

A summary of the variable ranges is shown in Table 5.3. In the table, the constraining platform motion is listed beside the variable range for each configuration. Thus for the 5-6-7 configuration, cant and twist ranges are shown for dependent sweep, sweep and twist ranges are shown for dependent cant, and sweep and cant ranges are shown for dependent twist. As seen in the table, the 3-DOF wing modules have sweep and cant variable ranges comparable to the 4 -DOF modules, however we see that in all cases, the twist range has be severely reduced. This once again is due to the orientation of the constraint planes. For the 3-DOF modules, there is always a combination of leading or trailing edge, upper or lower, and diagonal passive branches, thus forming a triangular constraint between the spherical joints. The limitations associated with diagonally opposing constrained branches, here, are compounded with the additional constraint, thus further reducing the effective twist rage for each 3-DOF configuration. The constraint planes could be reoriented to increase the twist range, but this could have an affect on other performance parameters not addressed here. What is apparent is the similarity in variable ranges depending on the constraint branch combination. This will be explored further below, but the variable range exhibits similarities with the 5-6-7 and 5-6-8 configurations, and with the 5-7-8 and 6-7-8 configurations. These two 
groupings are those with two constrained branches at the trailing and leading edge wing regions, respectively.

The distribution of platform variables depicted in the figures are grouped according to the variable range and the dependent motion. Thus the sweep distribution is depicted in Fig. 5.9 and Fig. 5.10 are associated with dependent cant and twist respectively. Likewise, Fig. 5.11 and Fig. 5.12 depict the cant distribution with dependent sweep and twist, respectively, and Fig. 5.13 and Fig. 5.14 depict the twist distribution with dependent sweep and cant, respectively. Like the kinematic range for the 4-DOF modules, the coloured contours and colourbars are representative of the particular platform variable variation of the wing module. For example, the coloured contours and colourbar for Fig. 5.9 are representative of the sweep maximum and minimum values of the cant-constrained wing module with respect to the module twist and span. Figure 5.10 shows the maximum and minimum sweep contours of the twist-constrained wing module with respect to the module cant and span. The remaining figures follow a similar convention for the cant and twist variation.

The constraint branch combinations between the pairing of trailing edge verse leading edge combinations is apparent for the sweep variation with both constrained platform cant and twist. Figure 5.9 shows the sweep variation for a constrained cant platform airfoil. Mirroring of the workspace is seen with the 5-6-7 (Fig. 5.9(a)) and 5-6-8 (Fig. 5.9(b)) configurations, and with the 5-7-8 (Fig. 5.9(c)) and 6-7-8 (Fig. 5.9(d)) configurations, respectively. Both groupings of trailing and leading edge configurations mirror variable ranges with respect to the change in twist sign. Additionally, we see that minimum and maximum sweep values occur at the same sweep sign, and are highly dependent on the module span, with positive (forward) sweep occurring at shorter module spans, and negative (aft) sweep occurring at large module spans. This is beneficial from an implementation point of view as additional trailing edge clearance is required for negative sweep, and larger module spans accommodate this requirement. We do see though that the twist range is limited due to the three constrained branches as discussed above.

The sweep variation for a constrained platform twist is shown in Fig. 5.10. Here, trailing edge 


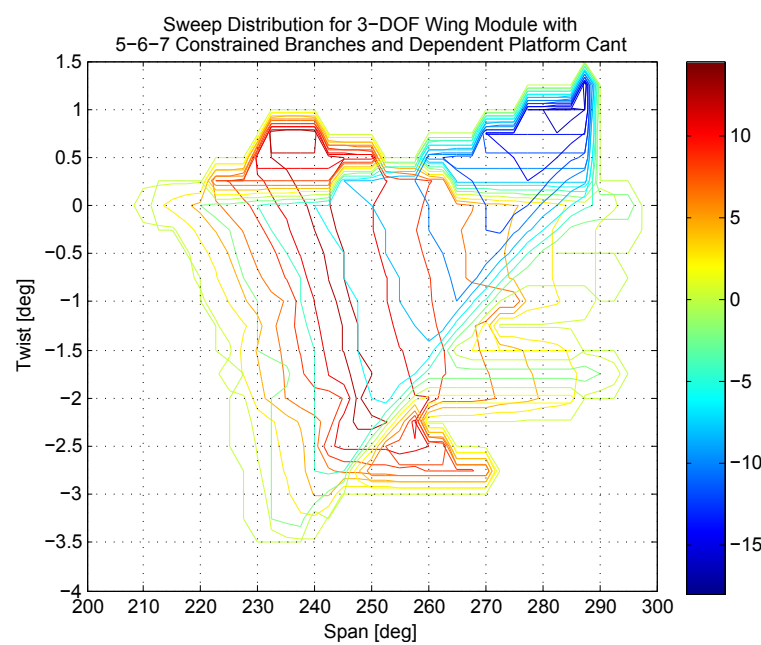

(a) 5-6-7 Constrained Branches

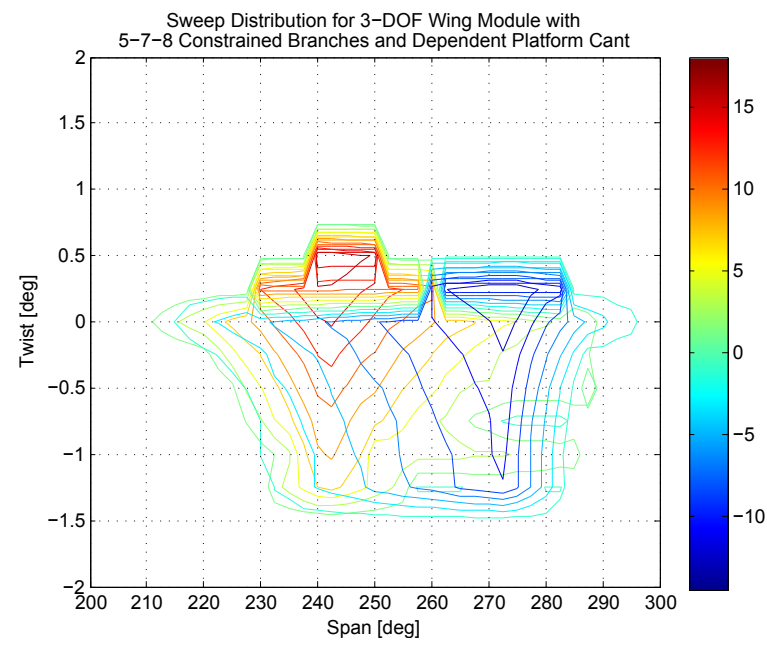

(c) 5-7-8 Constrained Branches

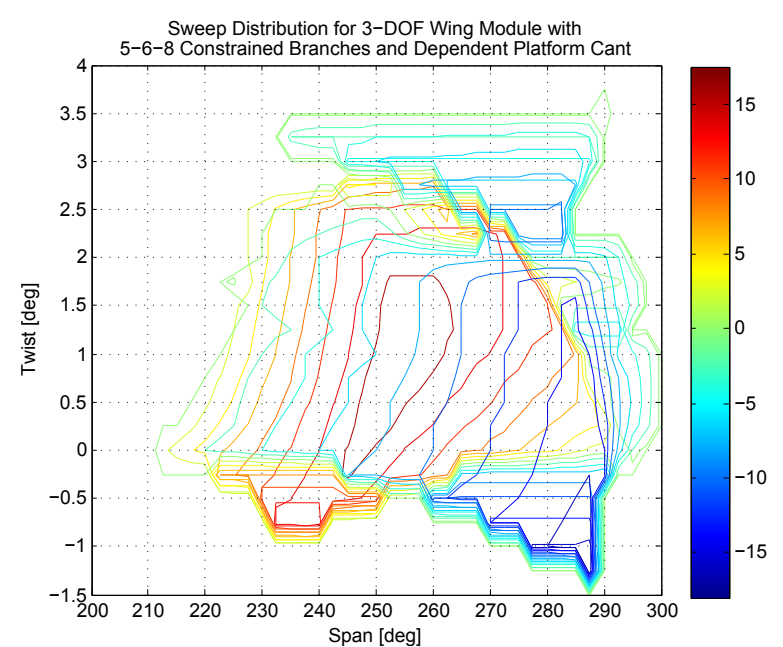

(b) 5-6-8 Constrained Branches

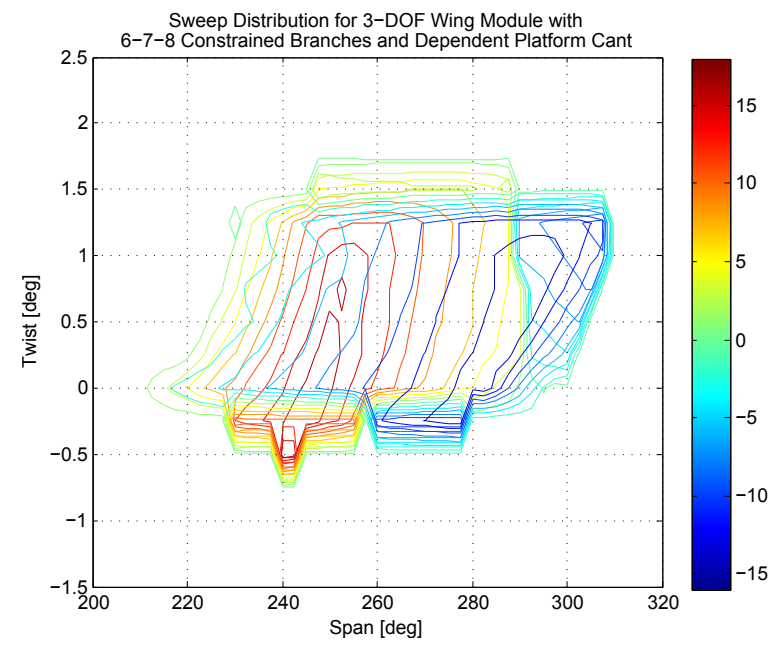

(d) 6-7-8 Constrained Branches

Figure 5.9: Sweep range of 3-DOF wing modules with constrained platform cant

(Fig. 5.10(a) and Fig. 5.10(b)) and leading edge (Fig. 5.10(c) and Fig. 5.10(d)) constrained branch groupings are evident from workspace shapes, where here, the workspaces are almost identical. The maximum positive sweep at smaller module spans and minimum negative sweep at larger module spans are maintained for the twist-constrained configurations, which as mentioned, is beneficial. The small variations in the ranges are associated with the asymmetric layout of branches, however the difference here are minor. What we do note for both the cant and twist-constrained configurations, the bulk of module sweep variable range can be achieved for a large range of twist and cant, respectively. 


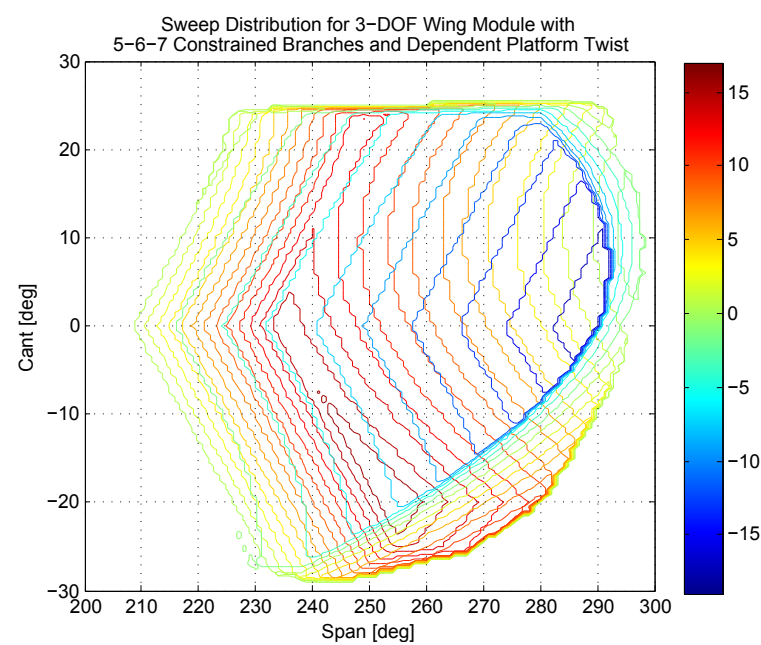

(a) 5-6-7 Constrained Branches

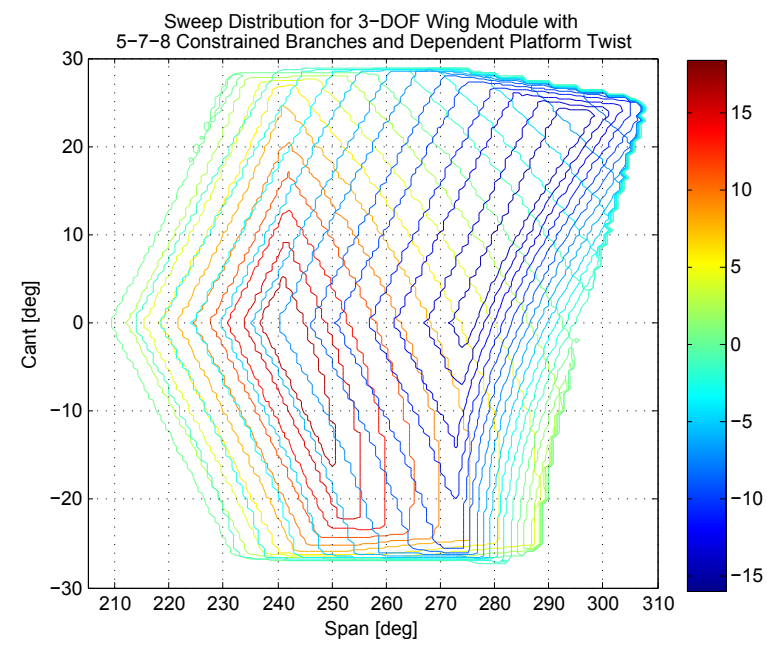

(c) 5-7-8 Constrained Branches

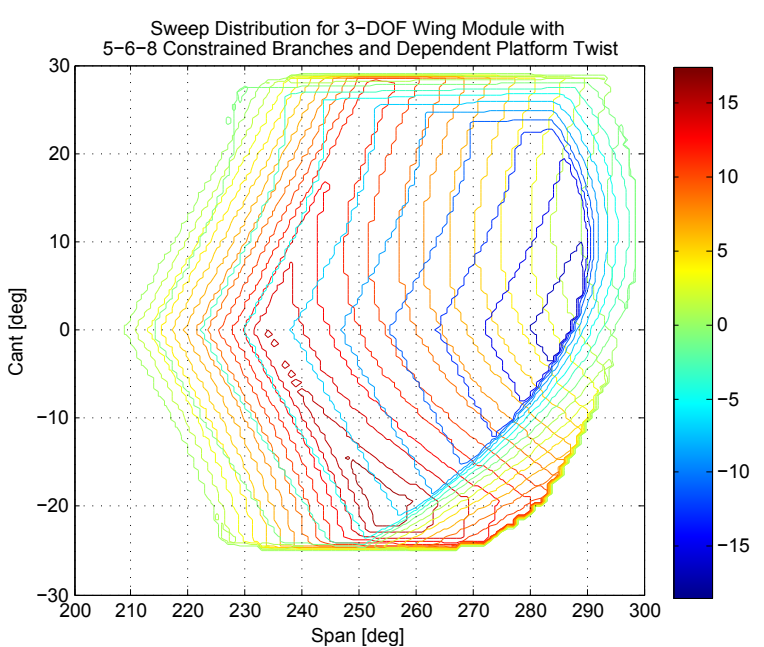

(b) 5-6-8 Constrained Branches

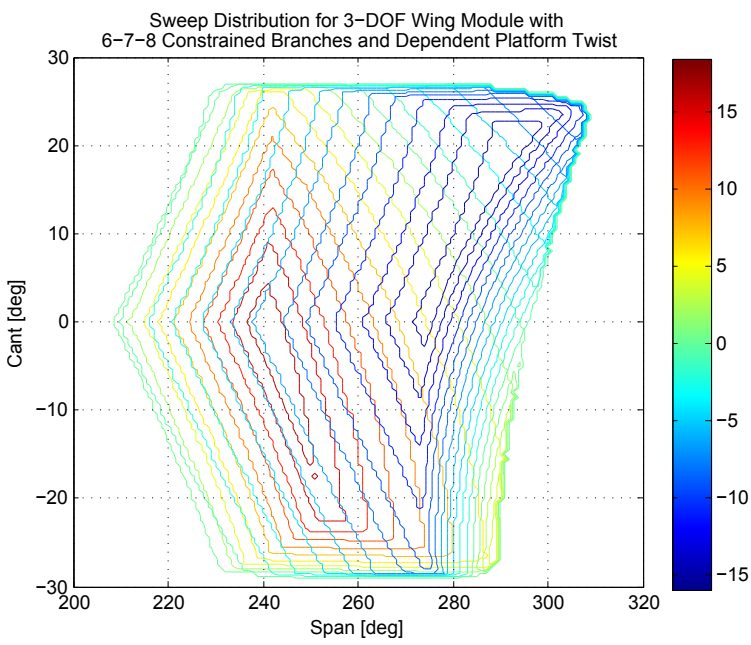

(d) 6-7-8 Constrained Branches

Figure 5.10: Sweep range of 3-DOF wing modules with constrained platform twist

As with the sweep, the trailing and leading edge constraint branch combination groupings are evident for the cant variation of the 3-DOF wing module configurations. For the sweep-constrained modules, there is again an apparent mirroring of the cant range shape with respect to a twist sign change, with minimum and maximum values being sided with respect to the sign of the platform twist as seen in Fig. 5.11. The 5-6-7 (Fig. 5.11(a)) and 5-6-8 (Fig. 5.11(b)) configurations show a large dependence on the cant range based on the span of the module, where negative cant values are achievable at smaller module spans, and positive cant values achievable at large module spans. Unlike the sweep variation, clearance requirements are not an issues with respect to cant variation, 


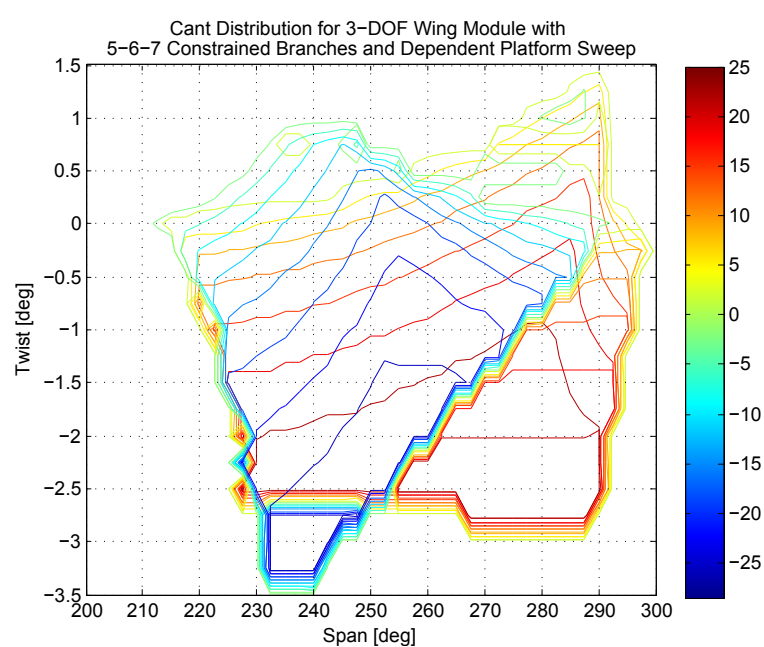

(a) 5-6-7 Constrained Branches

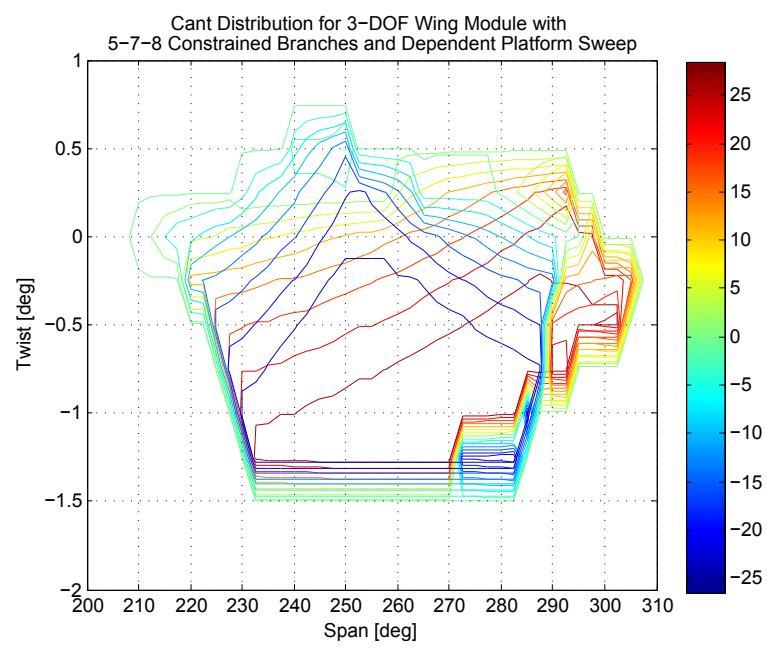

(c) 5-7-8 Constrained Branches

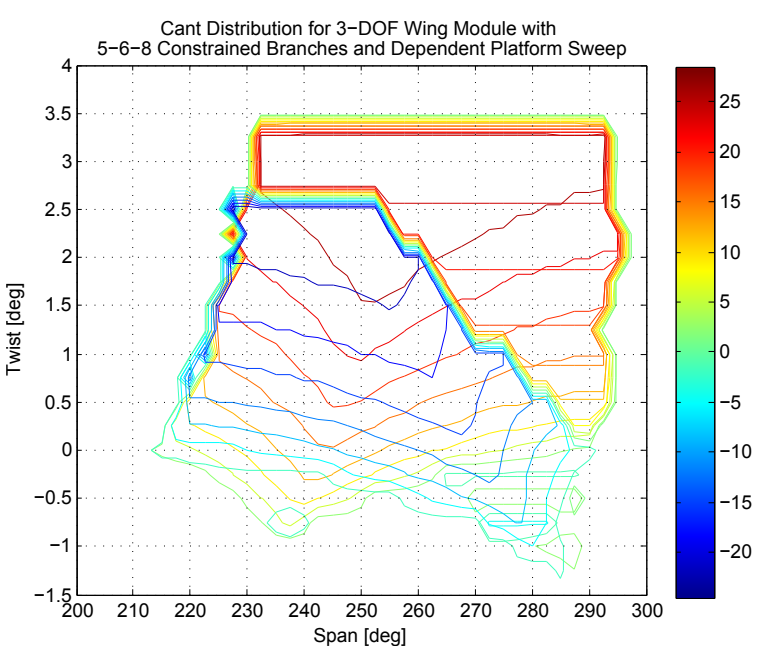

(b) 5-6-8 Constrained Branches

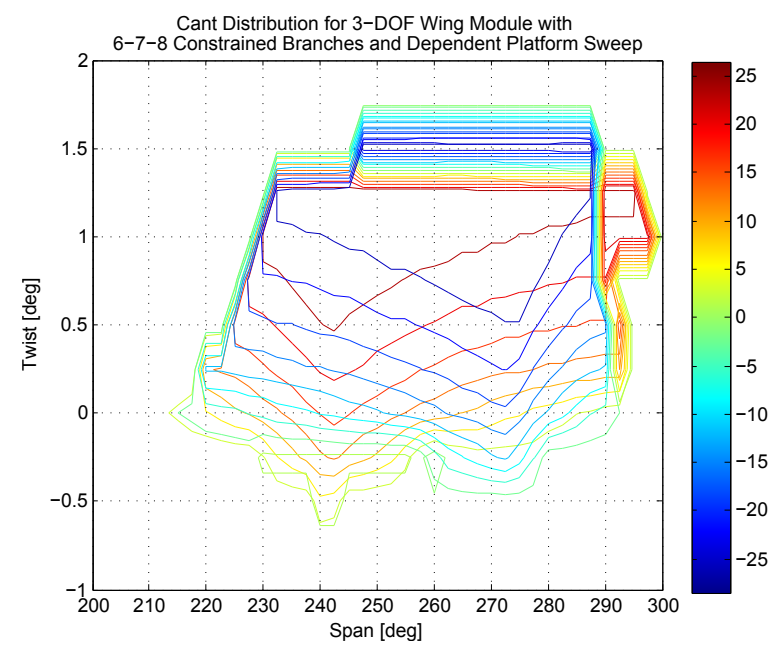

(d) 6-7-8 Constrained Branches

Figure 5.11: Cant range of 3-DOF wing modules with constrained platform sweep

so the variable range associated with the 5-6-7 and 5-6-8 constrained branch configurations are not necessarily a positive feature. This is in contrast though to the 5-7-8 (Fig. 5.11(c)) and 6-7-8 (Fig. 5.11(d)) constrained branch configurations where there the span-dependency on cant is less apparent. The minimum and maximum cant values are dependent though on the sign of the twist, but a larger span range is available in these instances, thus making the 5-7-8 and 6-7-8 configuration performance more desirable than the 5-6-7 and 5-6-8 configurations.

The cant variation for a constrained platform twist is shown in Fig. 5.12. Similarly to the sweep 


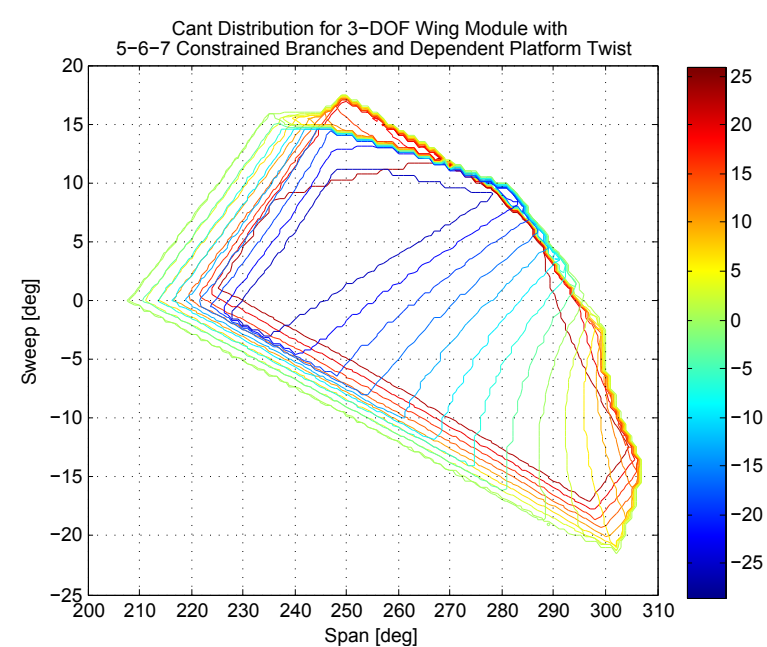

(a) 5-6-7 Constrained Branches

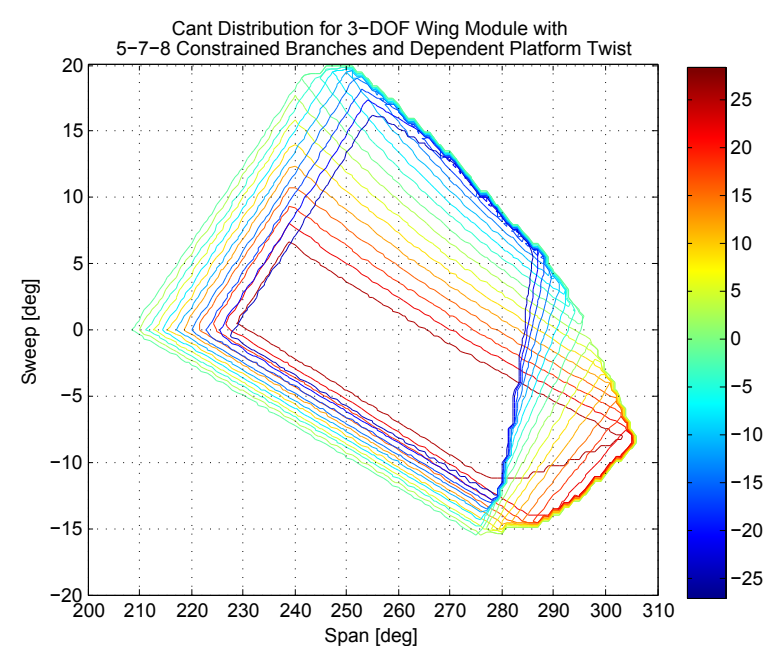

(c) 6-7-8 Constrained Branches

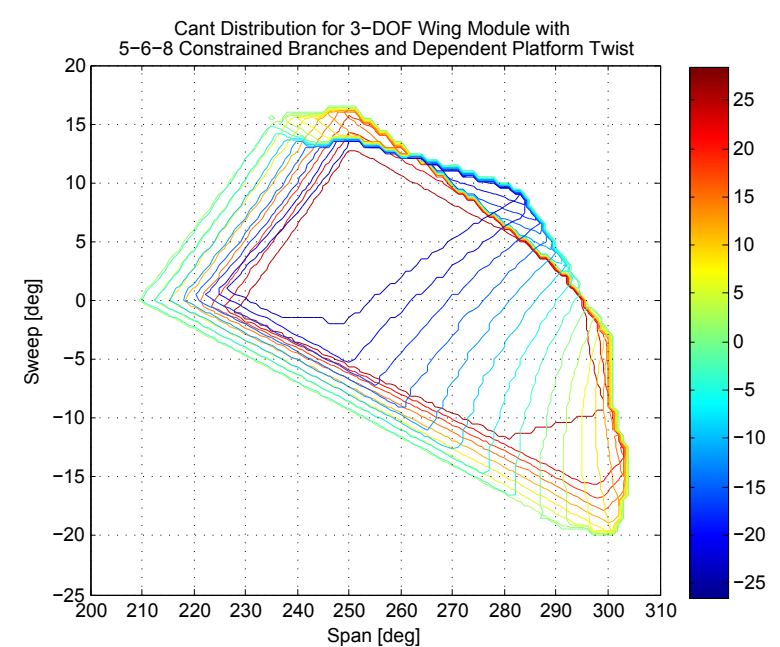

(b) 5-6-8 Constrained Branches

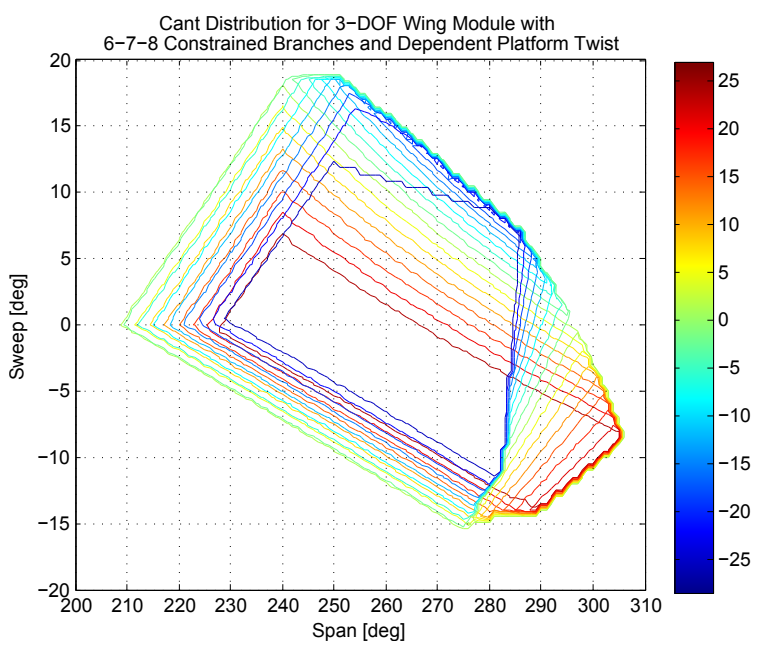

(d) 6-7-8 Constrained Branches

Figure 5.12: Cant range of 3-DOF wing modules with constrained platform twist

variation for a constrained platform twist, the workspace shapes here are almost identical for the trailing and leading edge constraint branch combinations. For these configurations, we see that there is a larger workspace for a positive cant for the 5-6-7 (Fig. 5.12(a)) and 5-6-8 (Fig. 5.12(b)) configurations, whereas the 5-7-8 (Fig. 5.12(c)) and 6-7-8 (Fig. 5.12(d)) configurations allow for a larger negative cant workspace. With respect to span variation, the minimum and maximum cant values generally are achievable near the mid-span region, however, as mentioned, the minimum and maximum values cover a large range of the variable workspace. This is in contrast to the sweepdependent configurations, where the 5-7-8 and 6-7-8 configurations exhibit a better variable range. 


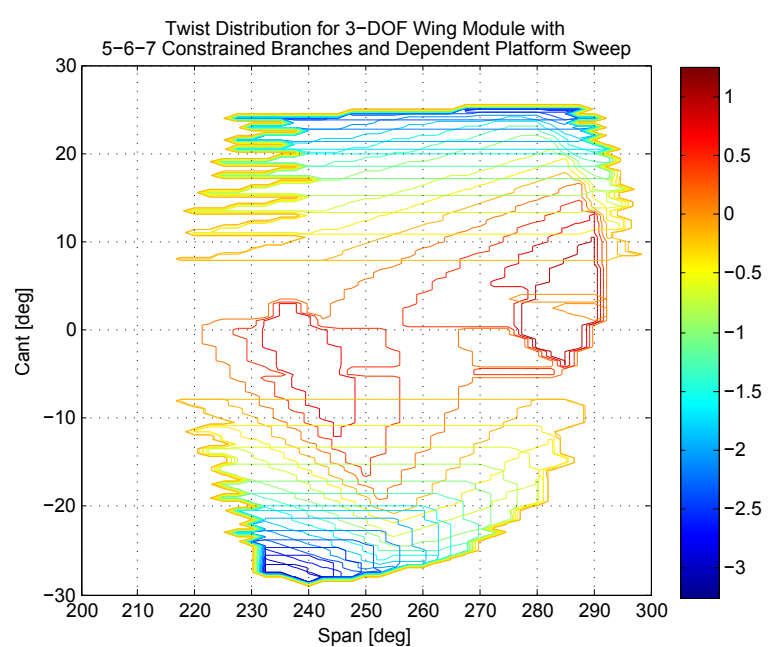

(a) 5-6-7 Constrained Branches

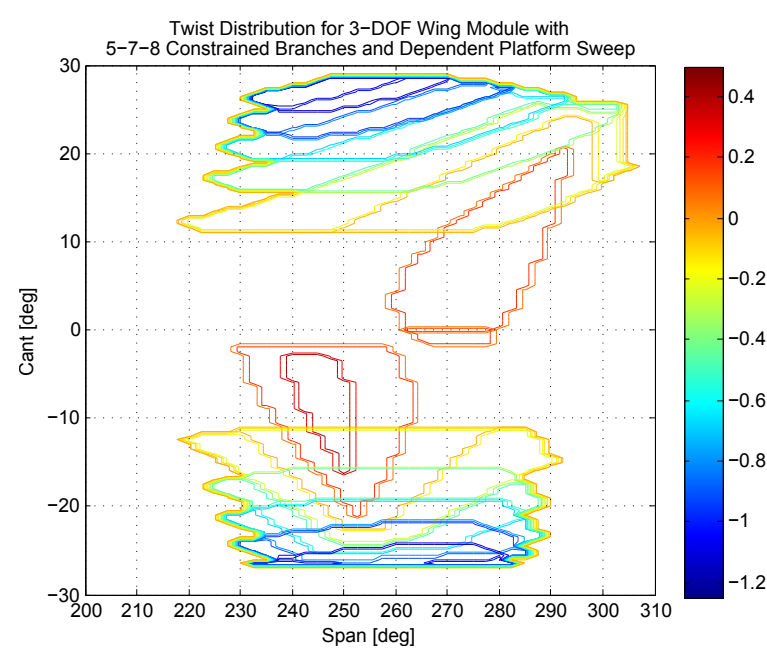

(c) 5-7-8 Constrained Branches

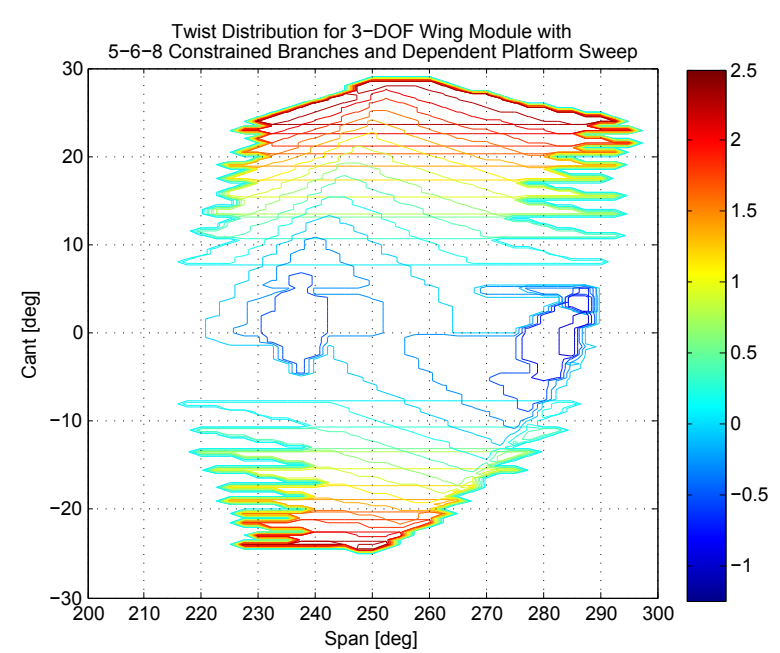

(b) 5-6-8 Constrained Branches

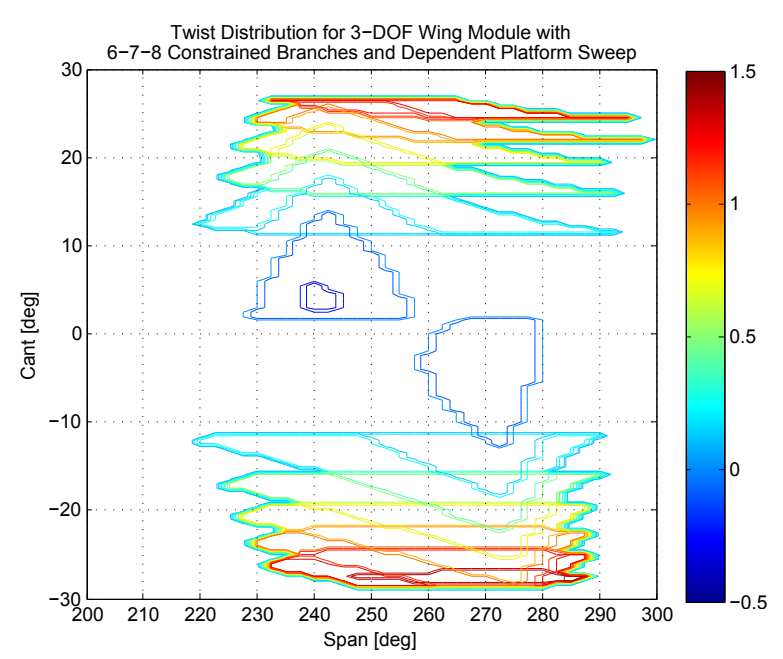

(d) 6-7-8 Constrained Branches

Figure 5.13: Twist range of 3-DOF wing modules with constrained platform sweep

Here, all four twist-dependent constraint branch combinations are good candidates with respect to the cant variable range, however if used in combination with a sweep-dependent module, the 5-7-8 and 6-7-8 configurations are best.

The twist variation for the sweep-dependent and cant-dependent modules are shown in Fig. 5.13 and Fig. 5.14, respectively. Once again, the trailing and leading edge constraint branch combination trends continue. The sweep-dependent configurations show workspaces that are essentially divided with respect to the sign of the module cant. The central portion of the workspace near $\Gamma=0^{\circ}$ 
is reachable, however due to the rendering of contours in Matlab, this region appears outside of the workspace. However, from the kinematics analysis, it is known that this workspace region is reachable. The twist workspace emerges further at cant values above/below $\Gamma= \pm 10^{\circ}$, with minimum and maximum twist values achievable at the cant workspace extremities. Here, we see that maximum and minimum twist values are essentially mirrored for the 5-6-7 (Fig. 5.13(a)) and 5-6-8 (Fig. 5.13(b)), and the 5-7-8 (Fig. 5.13(c)) and 6-7-8 (Fig. 5.13(d)) configurations, respectively. As the 5-6-7 and 5-6-8 configurations have a large twist variable range for $\Gamma \leq \pm 10^{\circ}$, these two configurations are better, and should be selected base on the minimum or maximum twist requirements of the module. There is a small dependence on module span.

Finally, the twist variation for the cant-dependent modules continue the workspace trends for the other 3-DOF configurations. The trailing edge (Fig. 5.14(a) and Fig. 5.14(b)) and leading edge (Fig. 5.14(c) and Fig. 5.14(d)) have similar but mirrored workspaces with respect to the maximum and minimum twist values. Here though, unlike the sweep-dependent configurations, the central portion of the workspace is well defined, thus all configurations can be used for morphing within this variable region. The twist variable extremities however are sided, with maximum or minimum values achievable at positive or negative sweep values. For these configurations, the twist variable extremities are dependent on the module span, however the majority of the twist range can be achieved within the bulk of the span and sweep variable ranges. As such, each module provides good kinematic performance. If used in combination with a sweep-dependent module, the the 5-6-7 or 5-6-8 configuration should be used. Again, the small variations in the workspaces are due to the asymmetric setup of the wing module branches.

The 3-DOF wing modules can achieve a very good kinematic range for the various configurations. As previously mentioned, the twist-dependent configurations are ideal due to the orientation of the constraint planes. Further work into the orientation of the constraint planes could result in larger twist variation for the sweep and cant-dependent configurations, yet this was not explored here. Exploring this kinematic feature should be done in combination with other pertinent module performance parameters to ensure an enhanced kinematic profile does not significantly degrade 


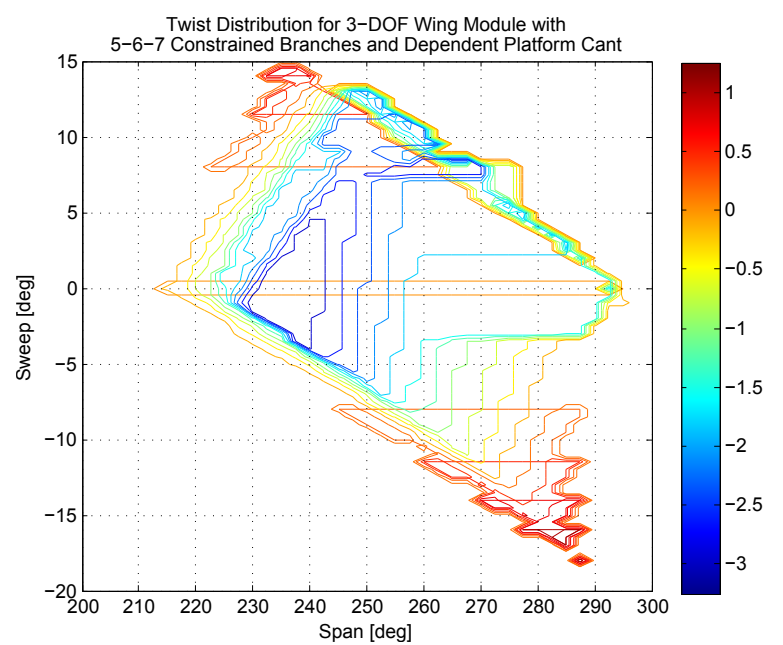

(a) 5-6-7 Constrained Branches

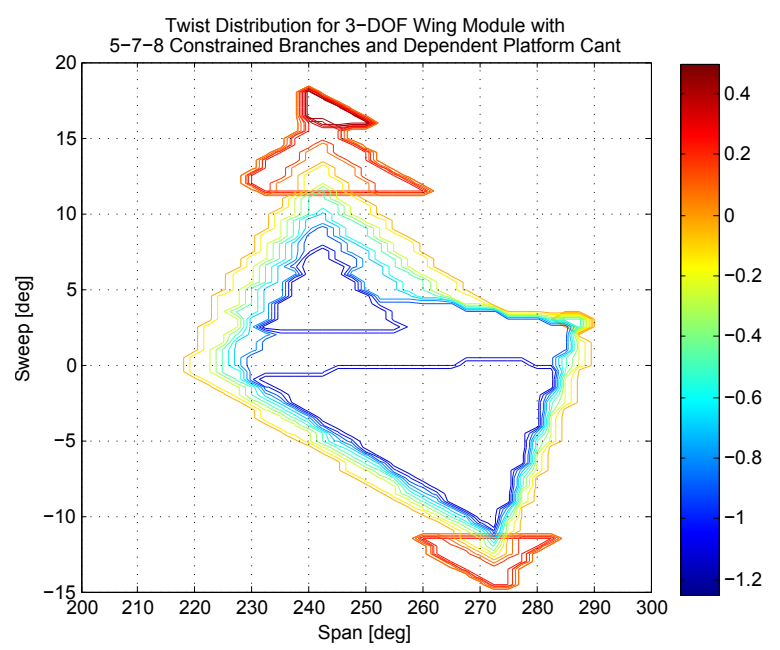

(c) 5-7-8 Constrained Branches

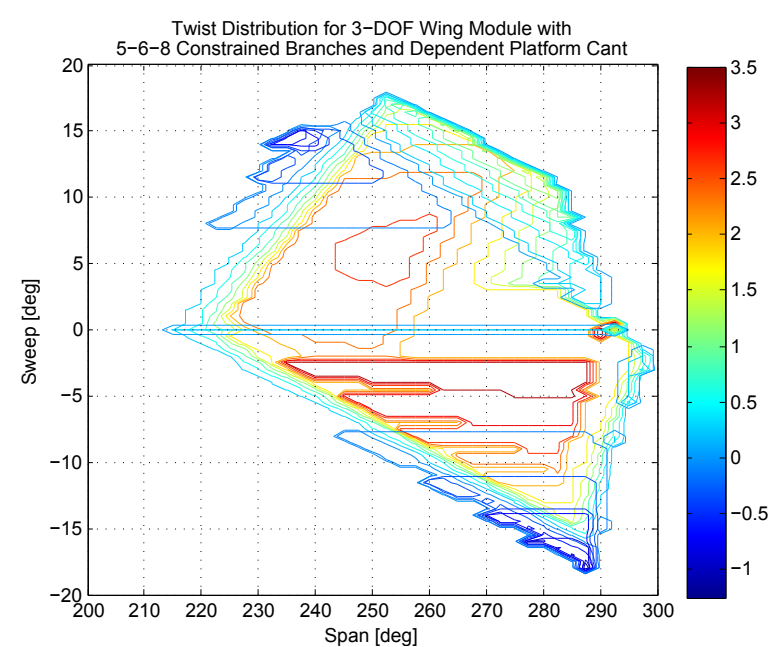

(b) 5-6-8 Constrained Branches

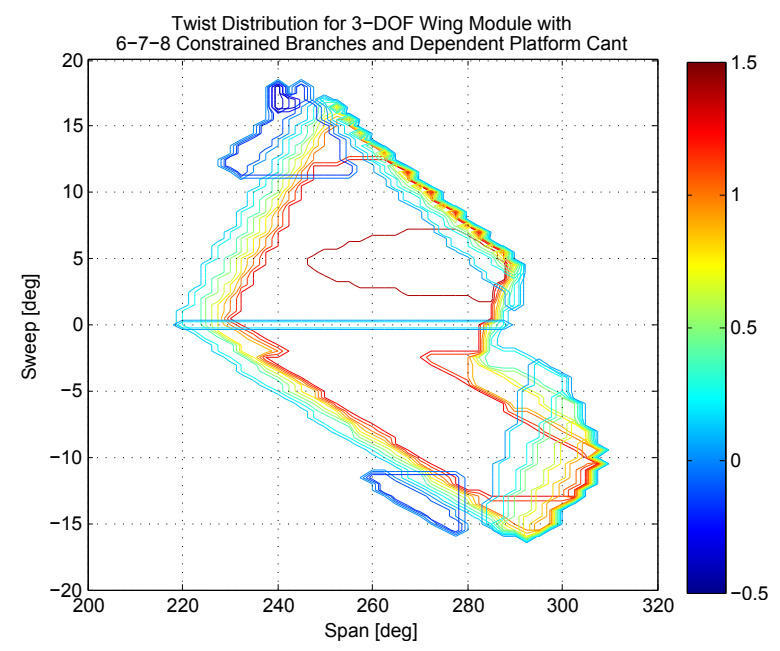

(d) 6-7-8 Constrained Branches

Figure 5.14: Twist range of 3-DOF wing modules with constrained platform cant

other parameters. An example of such could be the strength or stiffness of the wing truss under load. Orientation of the constraint planes to pass through the base airfoil $z$-axis is common among parallel robots, and thus was used here.

What is apparent from the figures above, are the similarities between the various 5-6-7 and 5-6-8, and 5-7-8 and 6-7-8 configurations, respectively. These grouping represent the configurations with two constrained branches at the trailing edge and leading edge, respectively. The other groupings which results in mirroring of minimum and maximum values are the 5-6-7 and 6-7-8, and the 5-6-8 
and 5-7-8 configurations. This grouping is for a pair of constrained branches on the upper and lower wing surfaces, respectively. Thus for these modules, larger variable ranges are sided between the trailing and leading edge combinations, whereas the mirroring of variable range is associated with the upper and lower wing surfaces. This knowledge allows the appropriate configuration to be selected based on the morphing requirements of the particular module.

\subsubsection{2-DOF Module Kinematic Range}

For the 2-DOF wing module, only one constrained branch configuration is possible, where all four parallel branches are constrained. As such, there is no choice in configuration selection for the 2-DOF wing modules. The only selection possible is which platform rotation is independent, rendering the remaining two dependent. The range of platform variables are listed in Table 5.4 and the span-wise distribution is shown in Fig 5.15.

The variable ranges for the platform sweep and cant remain relatively large for the 2-DOF module, as compared to those for the 4-DOF and 3-DOF modules, however, slightly diminished here. Again, this is due to the overall motion of platform airfoil tending in the direction in which the constrained branch spherical joints are forced to move. For the 2-DOF modules, all four axial branches are constrained, hence a rectangular constraint between the spherical joints is presented. The limitations associated with the 3-DOF wing modules are present here and then compounded with the additional constrained branch. Reorienting the constraint planes in a circular fashion could increase the twist capabilities of the module as module twist is a motion essentially perpendicular to the current orientation of the constraint planes. If left in the current configuration, the

Table 5.4: Kinematic range for the 2-DOF wing module configurations

\begin{tabular}{lll}
\hline $\begin{array}{l}\text { Independent } \\
\text { Motion }\end{array}$ & $\begin{array}{l}\text { Platform Airfoil Variable Range } \\
\text { Maximum Angle }\end{array}$ & Minimum Angle \\
\hline$\Lambda$ & 12.6 & -15.2 \\
$\Gamma$ & 12.6 & -16.7 \\
$\alpha$ & 0.45 & -0.25 \\
\hline
\end{tabular}




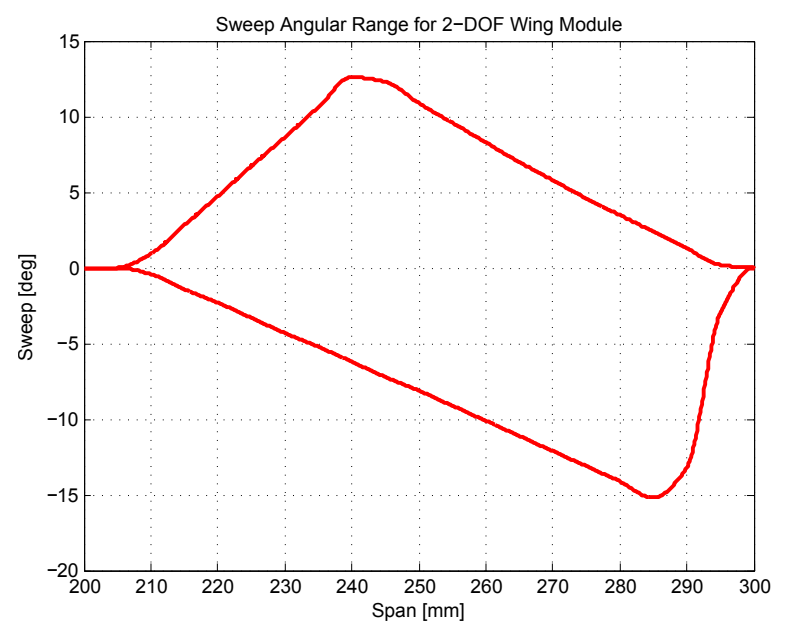

(a) Active Sweep

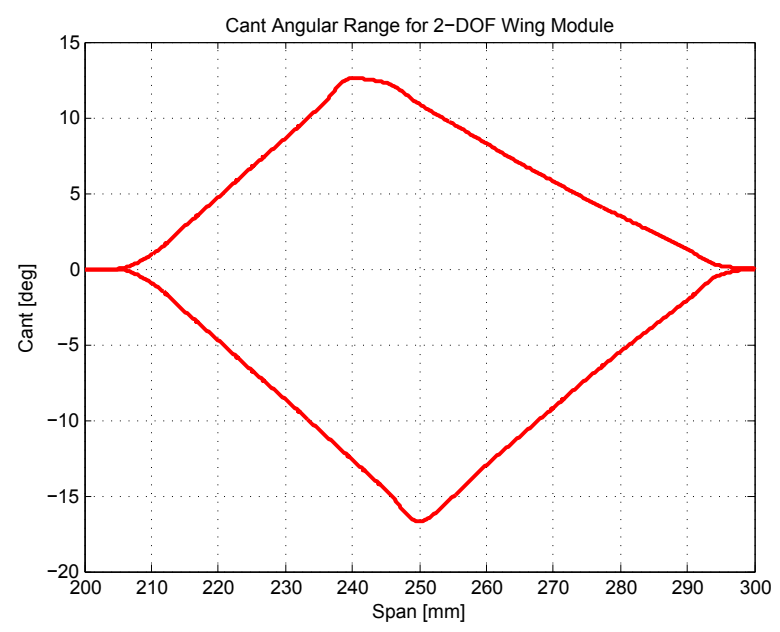

(b) Active Cant

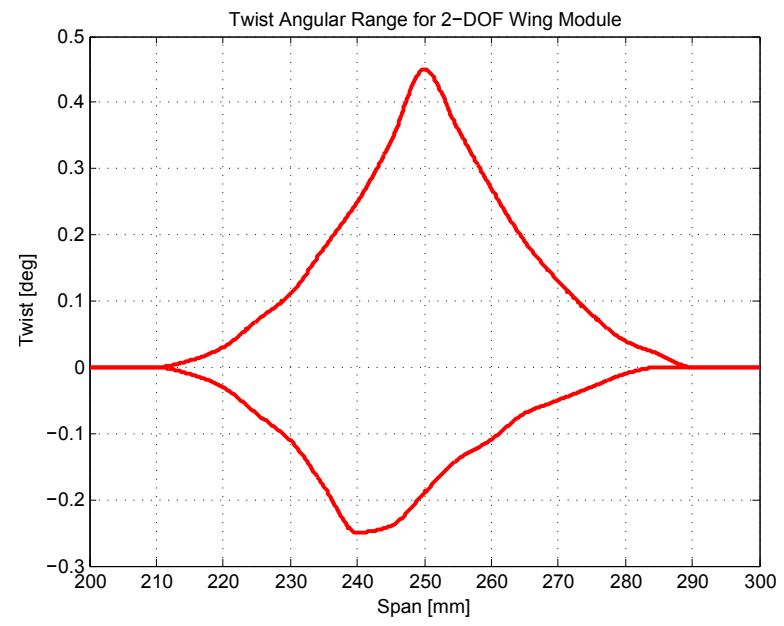

(c) Active Twist

Figure 5.15: Angular range of the 2-DOF wing module

limited twist motion would require sequential 2-DOF modules to achieve larger over wing twist values. Alternatively, increasing the mobility of module to a 4-DOF configuration with 5-6 or 7-8 constrained branches could overcome this, as two 2-DOF twist-capable wing modules are equally complex, with respect to the number of controllable degrees-of-freedom, as a single 4-DOF module.

Referring to Fig. 5.15(b), we see that the largest variable ranges occur primarily within the centre of the span limits of the module. For the cant and twist distributions, Fig. 5.15(b) and Fig. 5.15(c) show evidence of this, whereas the sweep distribution shown in Fig. 5.15(a) is slightly skewed. For a sweep-independent module, large positive sweep values are possible at shorter spans, whereas 
large negative sweep values occur at longer module spans. This is beneficial from the perspective of clearance, as a negative sweep indicates the wing is sweeping aft. The cant-independent module is less dependent on the module span as compared to the sweep-independent module. The cant extremities occur near the mid-span region, and taper off with both increasing and decreasing span. As clearance is not an issue for cant variation, the relative symmetry of the workspace is beneficial. Finally, the twist variation is very small. This is strictly due to four constrained branches within the system. As the constraint planes are essentially perpendicular to a twist motion, the platform does not have the freedom to reach large twist values.

\subsubsection{Module Constrained Jacobian Inverse Condition Distribution}

In addition to kinematics, the instantaneous kinematics of the wing module are used as a performance index for the various configurations. As the kinematics themselves varied according to the constrained branch combinations, the instantaneous kinematics depend not only on constrained branches within the system, but also on the active branches within the system. As such, there is further opportunity to tune the particular module configuration to meet the current requirements of wing.

The instantaneous kinematics here are evaluated by examining the constrained Jacobian inverse condition number. The condition of the Jacobian is an indication of how close a parallel mechanism is to being isotropic or singular. A parallel mechanism, including the wing module is isotropic if both $\mathbf{J}_{f w d}$ and $\mathbf{J}_{i n v}$ have a condition number, $\mu$ equal to 1 at a minimum of one pose within the workspace [72]. The closer the condition number is to unity, the closer the hyperellipsoid of platform motions is to being a hypersphere; a desirable quality for velocity and force transmission purpose [73]. Alternatively, the closer the condition number is to infinity, the close the robot is to being in a singular position. Study has been done recently on Jacobian conditioning, where global indices, distribution have been discussed with respect to parallel robots [74]. Although somewhat

disputed, the condition distribution of the Jacobian can be used to identify singular positions within the workspace [51]. 
Indices used here for comparative purposes are defined using the inverse condition number $1 / \mu$, which falls on the interval $[0,1]$. An inverse condition number value of 0 indicates a singular constrained Jacobian and a value of 1 indicates an isomorphic configuration. As the condition number varies throughout the workspace, a sample of values are taken throughout the entire workspace. For each case, the maximum, and non-singular minimum, average and standard deviation of the the inverse condition is given. These values act as a gauge on the performance of the wing module configurations.

\subsubsection{4-DOF Jacobian Inverse Condition Distribution}

The six configurations of the 4-DOF wing module consists of the four active branches being combined with two constrained branches, leading to six possible configurations. As with the kinematic evaluation of the 4-DOF configuration, visualization of the constrained Jacobian inverse distribution requires manifolds in 4-space. The distribution presented in Fig. 5.16 is representative of the module at $b_{z}=250 \mathrm{~mm}$. The summary of condition data is given in Table 5.5.

The constrained Jacobian inverse condition maximum, non-singular minimum, average and standard deviation listed in Table 5.5 show that the 5-6 and 7-8 constrained branch configurations exhibit better performance as compared to the remaining configurations. As all four diagonal

Table 5.5: Constrained Jacobian inverse condition data for the 4-DOF wing module configurations at $b_{z}=250 \mathrm{~mm}$

\begin{tabular}{lllll}
\hline $\begin{array}{l}\text { Constrained } \\
\text { Branches }\end{array}$ & \multicolumn{4}{l}{ Constrained Jacobian Inverse Condition } \\
\hline $5-6$ & $\boldsymbol{\mu}_{\max } \times \mathbf{1 0}^{-\mathbf{4}}$ & $\boldsymbol{\mu}_{\min } \times \mathbf{1 0}^{-\mathbf{9}}$ & $\boldsymbol{\mu}_{\text {avg }} \times \mathbf{1 0}^{-\mathbf{4}}$ & $\boldsymbol{\mu}_{\text {std }} \times \mathbf{1 0}^{-\mathbf{4}}$ \\
$5-7$ & 50.647 & 29.433 & 18.850 & 13.715 \\
$5-8$ & 5.0066 & 64.123 & 2.2361 & 1.3035 \\
$6-7$ & 7.6377 & 9.8076 & 3.0022 & 1.8752 \\
$6-8$ & 6.9521 & 13.932 & 2.745 & 1.714 \\
$7-8$ & 0.9424 & 0.9157 & 0.19938 & 0.18485 \\
\hline
\end{tabular}


branches are used, this is solely due to the position and orientation of the constraint planes. For these two configurations, they have a relatively high maximum and average values for the workspace, which is a positive attribute. The remaining configurations, 5-7, 5-8, 6-7 and 6-8, each are at least an order of magnitude smaller for the maximum and average values as compared to the 5-6 and 7-8 configurations. The 6-8 configuration exhibits the poorest performance at two orders of magnitude smaller. With respect to the non-singular minimum values, the 5-7 configuration has the largest minimum, followed by the 5-6 configurations. Thus from the data in presented in Talbe 5.5, the 5-6 and 7-8 configurations both have the best performance.

Figure 5.16 shows the constrained Jacobian inverse condition distribution for the various 4-DOF configurations. From the analysis, the 5-6 (Fig. 5.16(a)) and 7-8 (Fig. 5.16(f)), the 5-7 (Fig. 5.16(b)) and 6-8 (Fig. 5.16(e)), and the 5-8 (Fig. 5.16(c)) and 6-7 (Fig. 5.16(d)) configurations show similar distributions. The 5-6 and 7-8 configurations represent the two constrained branches positioned at the trailing and leading edge, respectively. For these configurations, we see that the larger condition numbers are sided considerably towards the cant variation, whereas the sweep variation has little effect. Generally, the wing would require a positive cant, thus the 7-8 configuration show a high condition distribution in this region, but additionally, the negative cant condition distribution is high when compared to the distribution of the 5-6 configuration. For the remaining 4-DOF configurations, condition distribution is dependent on the module cant, and the distribution itself is almost equivalent for both positive and negative cant values. However as summarized in Table 5.5, the condition distribution of the 5-7, 5-8, and 6-7 are an order of magnitude smaller than that of the 5-6 and 7-8 configurations, with the 6-8 condition distribution two orders of magnitude smaller. This in combinations with the overall twist range, confirms the dominance of the 5-6 and 7-8 configurations with respect to the kinematic performance.

We can also see that there is a clear region where every constraint configuration is singular, or near singular. The dark streak in the centre of the workspace occurs when the sweep $\Lambda=0$, and cant $\Gamma=0$, for distinct values of twist. Specifically, the 5-6 and 7-8, the 5-7 and 6-8, and the 5-8 and 6-7 configurations have a singular line spanning twist values of $-22^{\circ} \leq \alpha \leq 22^{\circ},-1^{\circ} \leq \alpha \leq 1.5^{\circ}$, 


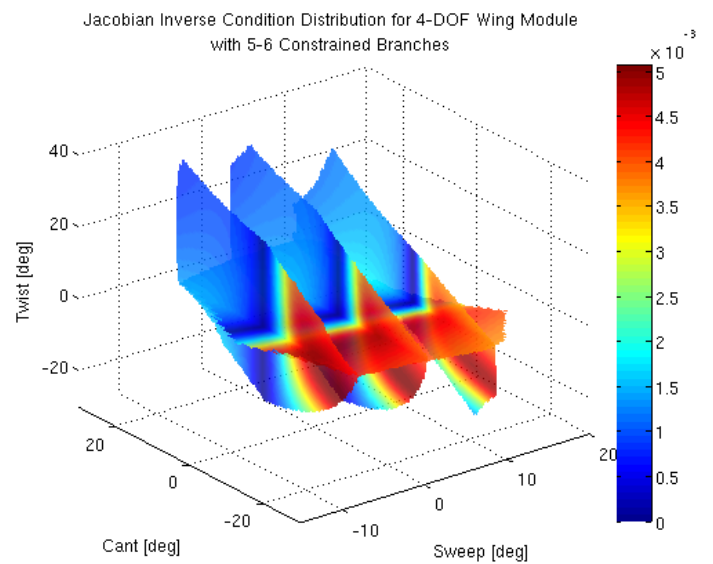

(a) 5-6 Constrained Branches

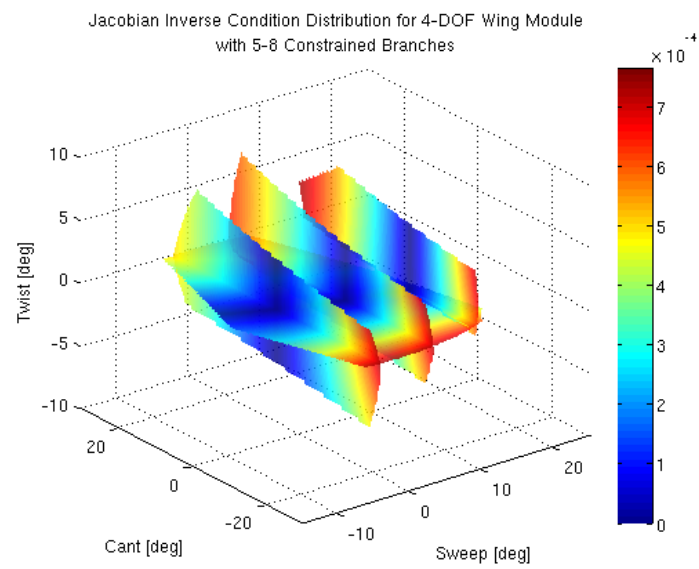

(c) 5-8 Constrained Branches

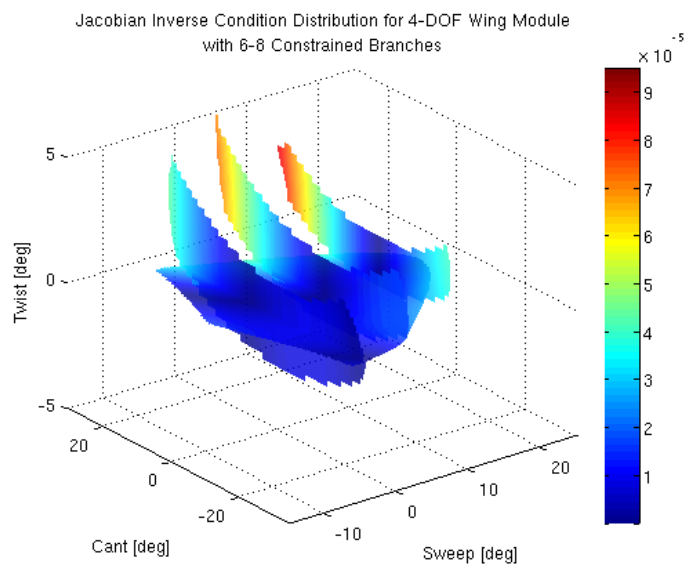

(e) 6-8 Constrained Branches

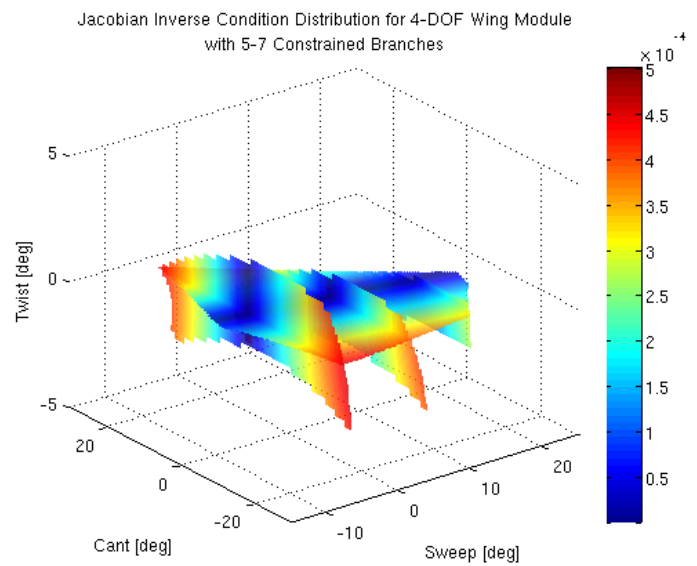

(b) 5-7 Constrained Branches

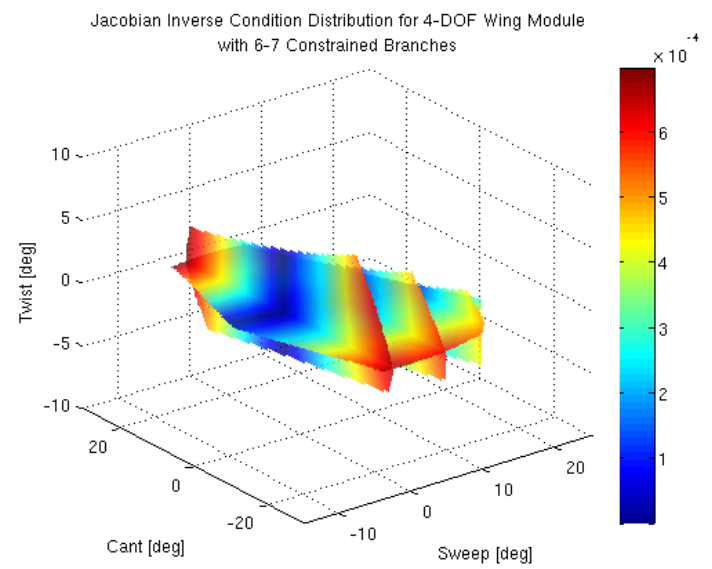

(d) 6-7 Constrained Branches

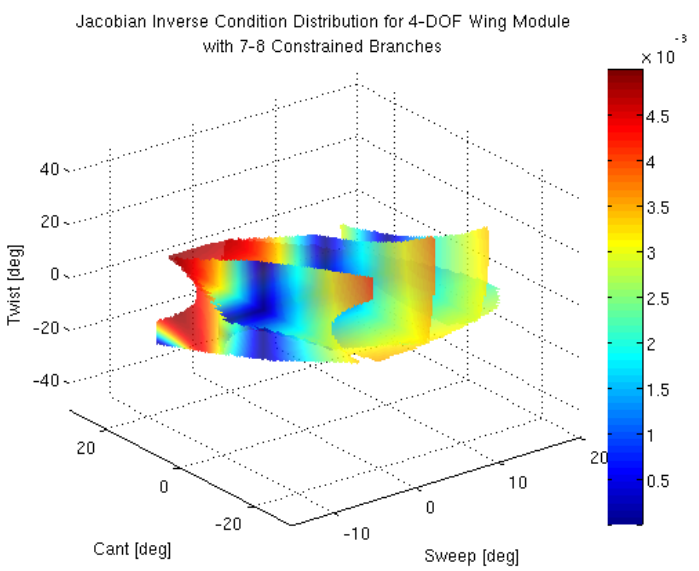

(f) 7-8 Constrained Branches

Figure 5.16: Constrained Jacobian inverse condition distribution for 4-DOF wing modules at $b_{z}=$ $250 \mathrm{~mm}$ 
and $-3.5^{\circ} \leq \alpha \leq 4.5^{\circ}$, respectively. Along these lines, the opposing active branches 1 and 3 , and/or 2 and 4, are equal in length and opposite in direction, which results in linear dependent rows of the constrained Jacobian. This reduces the matrix rank, and the constrained Jacobian is singular in these positions.

\subsubsection{3-DOF Jacobian Inverse Condition Distribution}

From the kinematic evaluation presented in Section 5.3.1.2, it is evident that the current configuration of constrained branches, i.e. their orientation with respect to the base airfoil coordinate frame, renders the 3-DOF wing module ideal to provide independent sweep and cant rotations, with a constrained twisting motion. Sweep or cant-constrained modules are indeed kinematically viable as suitable ranges shown in Table 5.3, however for comparative purposes here, the instantaneous kinematic analysis for the 3-DOF wing modules is limited to those that have dependent twist motion. Further reasons for limiting the analysis is due to the number of configurations that would need to be analyzed. The 3-DOF configurations each have three active and three passive branches. With four combinations of each as outlined in Table 4.8, a total of 16 module configurations are possible. If we were to also consider sweep and cant-constrained configurations, a total of 48 module configurations are possible. Instead, focus is placed on the twist-constrained modules so that a trends and comparisons can be made between these configurations. These wing modules are analyzed, and a summary of constrained Jacobian inverse condition data is presented in Table 5.6.

For the 3-DOF wing module constrained Jacobian inverse condition, there is a large selection of configurations from which not only to analyze the kinematic performance, but also to select which is best suited for a given task. In Table 5.6, the condition data is grouped according to the constrained branch configuration so that similar configurations can be compared. For the 5-6-7 and 5-6-8 configurations, we see from the data, the maximum and average condition values are largest for the 1-3-4 active branch configuration. These configurations have an active branch on the upper and lower wing surface, and at the leading edge. We see also that for the 5-6-7 constrained branch configurations, the non-singular minimum values are generally larger than the 5-6-8 configurations 
Table 5.6: Constrained Jacobian inverse condition data for the 3-DOF wing module configurations with dependent platform twist

\begin{tabular}{llllll}
\hline Passive & Active & \multicolumn{3}{l}{ Constrained Jacobian Inverse Condition } & \\
Branches & Branches & $\boldsymbol{\mu}_{\max } \times \mathbf{1 0}^{-\mathbf{3}}$ & $\boldsymbol{\mu}_{\min } \times \mathbf{1 0}^{-\mathbf{7}}$ & $\boldsymbol{\mu}_{\text {avg }} \times \mathbf{1 0}^{-\mathbf{3}}$ & $\boldsymbol{\mu}_{\text {std }} \times \mathbf{1 0}^{\mathbf{- 3}}$ \\
\hline $5-6-7$ & $1-2-3$ & 4.0004 & 1.3874 & 2.6209 & 1.0614 \\
& $1-2-4$ & 8.1239 & 61.535 & 3.3678 & 2.0482 \\
& $1-3-4$ & 10.816 & 24.8 & 3.693 & 2.1514 \\
& $2-3-4$ & 4.4702 & 29.072 & 2.796 & 1.0222 \\
$5-6-8$ & & & & & \\
& $1-2-3$ & 3.9867 & 1.8513 & 1.8898 & 1.111 \\
& $1-2-4$ & 7.2367 & 0.25298 & 3.2427 & 2.1721 \\
& $1-3-4$ & 11.194 & 0.48671 & 3.4989 & 2.3654 \\
& $2-3-4$ & 4.3345 & 1.4436 & 2.868 & 1.0659 \\
$5-7-8$ & & & & & \\
& $1-2-3$ & 4.1946 & 0.095915 & 1.3575 & 1.2659 \\
& $1-2-4$ & 7.2863 & 0.011803 & 3.1054 & 1.792 \\
& $1-3-4$ & 6.276 & 14.347 & 3.0329 & 1.6577 \\
& $2-3-4$ & 4.1834 & 0.70935 & 1.2606 & 1.0036 \\
$6-7-8$ & & & & & \\
& $1-2-3$ & 4.2717 & 0.56404 & 1.5961 & 1.2529 \\
& $1-2-4$ & 6.9322 & 1.4366 & 2.5984 & 1.7003 \\
& $1-3-4$ & 5.7155 & 14.223 & 2.4708 & 1.4413 \\
& $2-3-4$ & 3.8899 & 4.4022 & 1.5594 & 0.94849 \\
\hline
\end{tabular}

with the same active branches. For the 5-7-8 and 6-7-8 configurations, the maximum values are relatively close for each of the active branch configuration, but we see here than the largest nonsingular minimum values occur for the 1-3-4 active branch modules. This temporarily indicates that the 1-3-4 active branch configurations tend to outperform the other active branch configurations.

To further analyze this apparent trend, the condition distribution of the various configurations are analyzed. The condition distribution for the 5-6-7, 5-6-8, 5-7-8 and 6-7-8 constraint branch configurations are shown in Fig. 5.17, Fig. 5.18, Fig. 5.19, and Fig. 5.20 respectively. The condition distribution for these configurations all are highly dependent on the module cant, with less of a dependence on the span and sweep variation in the workspace. This phenomenon was also apparent with the $4-\mathrm{DOF}$ wing modules.

The constrained Jacobian inverse condition distribution for the 5-6-7 constraint branch configurations is shown in Fig 5.17. From the figure, we see that the 1-2-3 and 2-3-4 active branches 


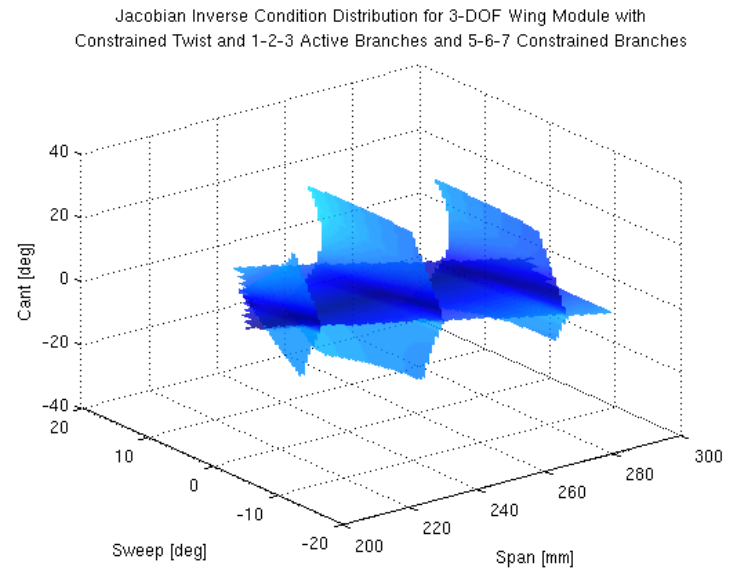

(a) 1-2-3 Active Branches

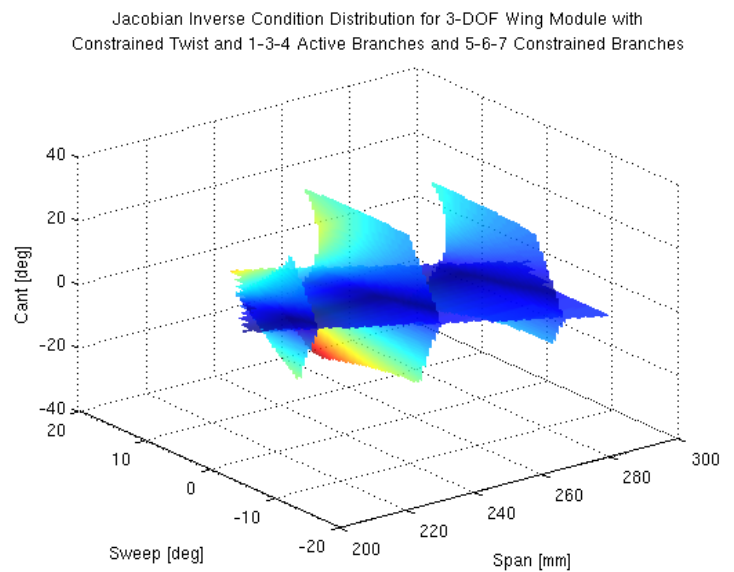

(c) 1-3-4 Active Branches

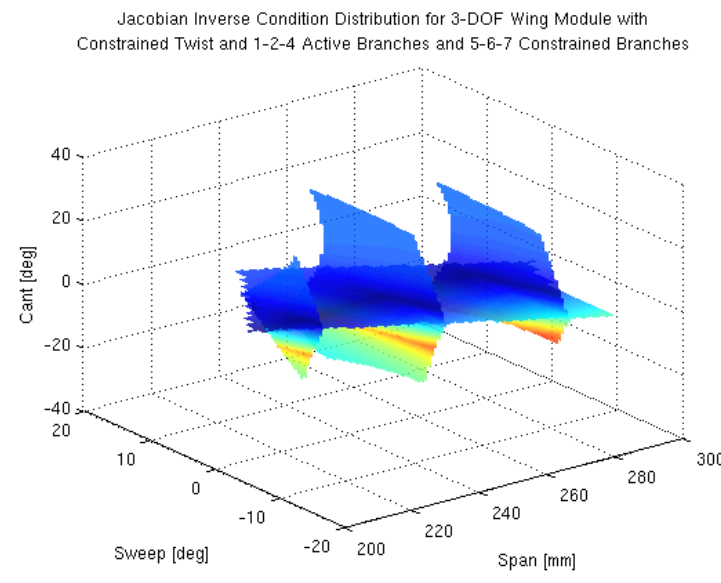

(b) 1-2-4 Active Branches

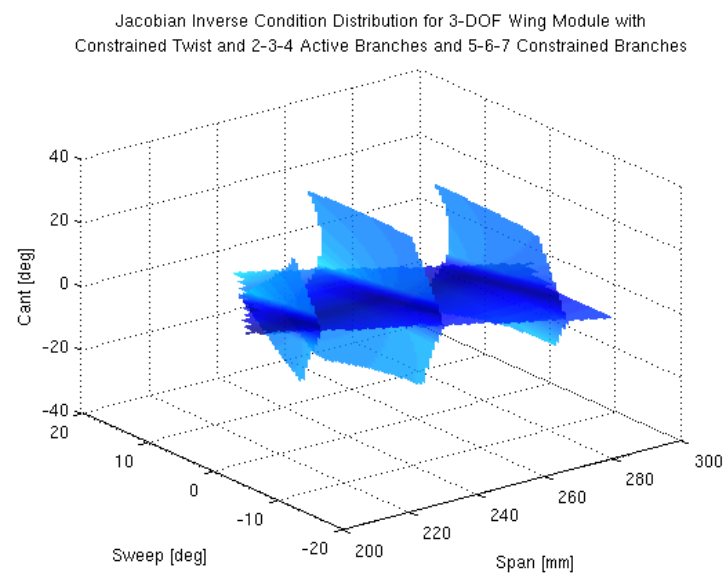

(d) 2-3-4 Active Branches

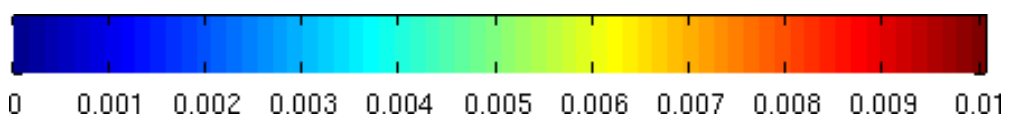

Figure 5.17: Constrained Jacobian inverse condition distribution for 2-DOF wing modules with constrained platform twist and 5-6-7 constrained branches

configurations each exhibit a condition distribution that is fairly low, and uniform throughout the workspace (see Fig. 5.17(a) and Fig. 5.17(d), respectively), which confirms the condition data presented in Table 5.6. The 1-2-4 (Fig. 5.17(b)) and 1-3-4 (Fig. 5.17(c)) configurations each show a greater variation in Jacobian condition that is highly dependent on the module cant, where negative cant values shown better Jacobian conditions. Conversely, we see that the module sweep has less of an effect on the condition for the 1-2-4 module, as compared to the 1-3-4 module where in addition to the negative cant, positive sweep values show better conditions. 


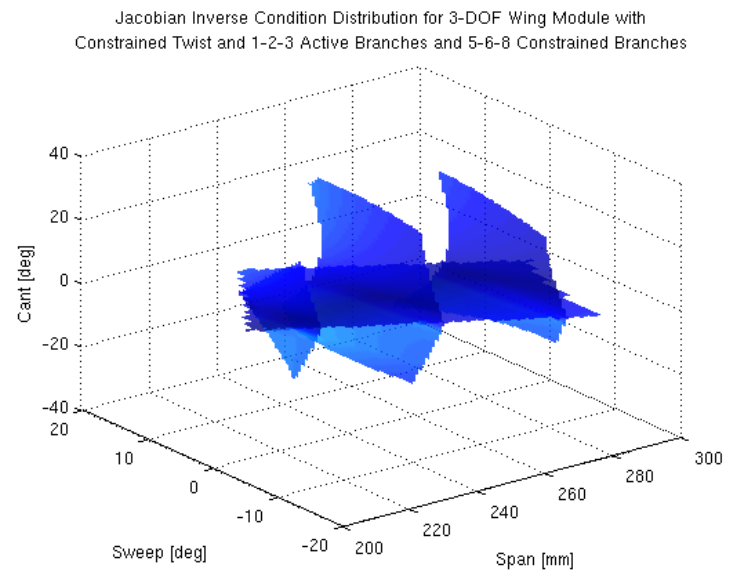

(a) 1-2-3 Active Branches

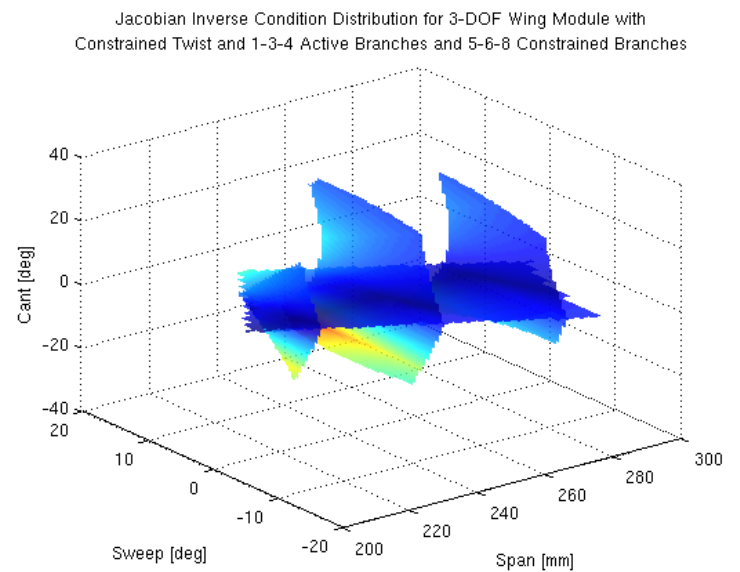

(c) 1-3-4 Active Branches

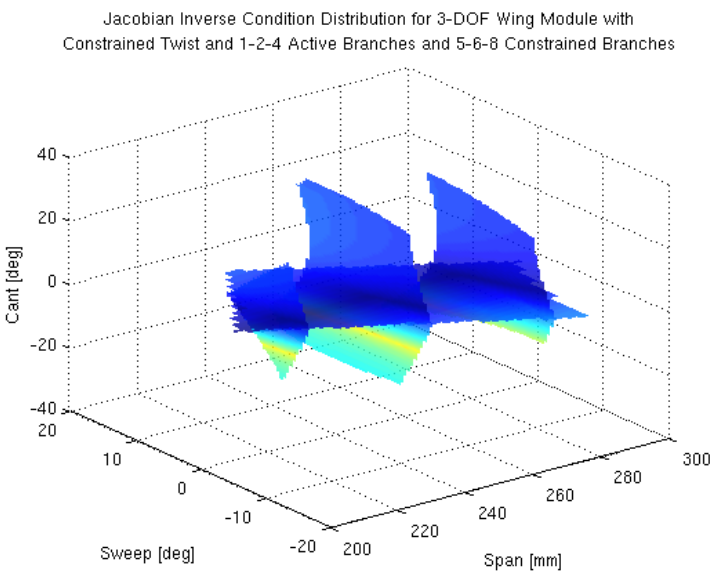

(b) 1-2-4 Active Branches

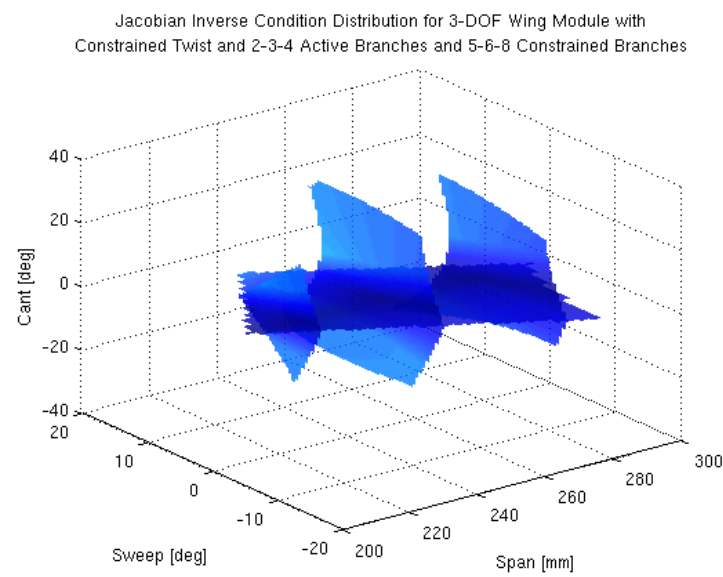

(d) 2-3-4 Active Branches

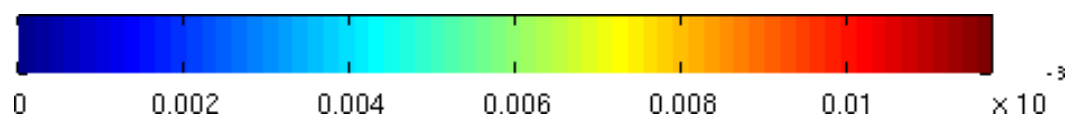

Figure 5.18: Constrained Jacobian inverse condition distribution for 2-DOF wing modules with constrained platform twist and 5-6-8 constrained branches

For the 5-6-8 constraint branch configurations, the condition distribution for the various active branch configurations shows very similar trends to those with 5-6-7 constrained branches. Figure 5.18 shows the distribution for the 5-6-8 constraint branches configurations which if compared to Fig. 5.17, the condition distribution is almost identical. The 1-2-3 (Fig. 5.18(a)) and 2-3-4 (Fig. 5.18(d)) configurations again show a consistently low condition over the workspace, while the 1-2-4 (Fig. 5.18(b)) and 1-3-4 (Fig. 5.18(c)) configurations show a condition distribution that is dependent on the module cant. For the 1-2-4 configuration, there is a band within the negative 


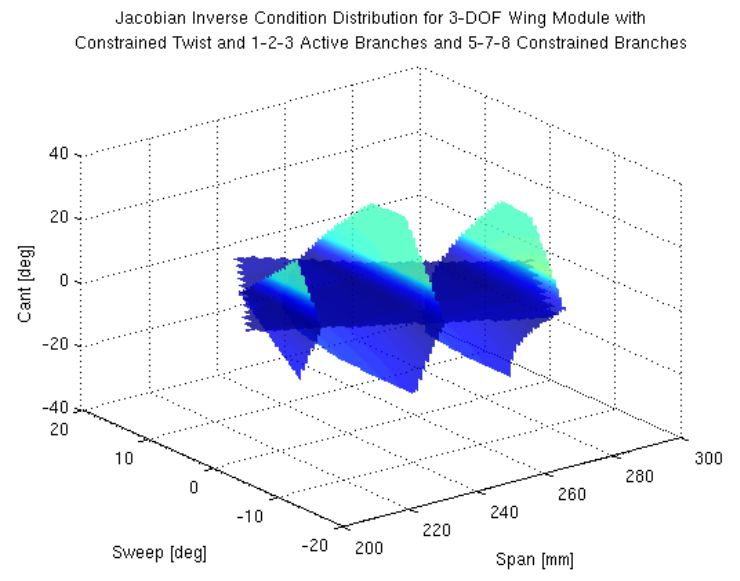

(a) 1-2-3 Active Branches

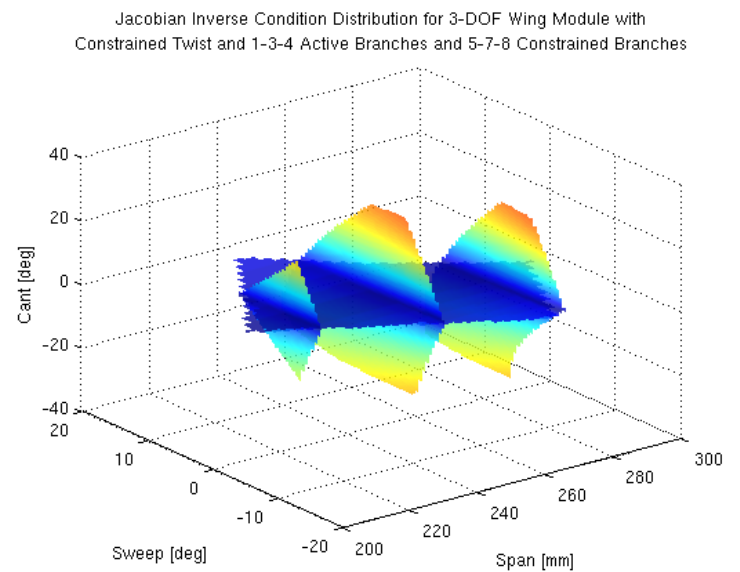

(c) 1-3-4 Active Branches

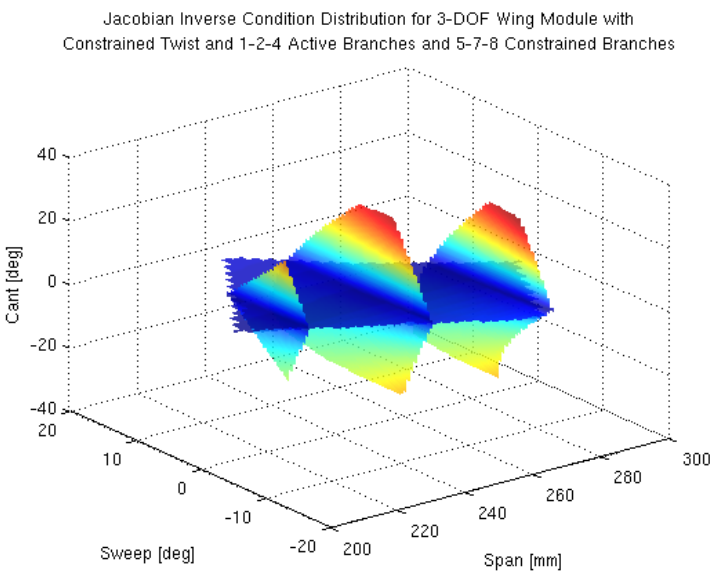

(b) 1-2-4 Active Branches

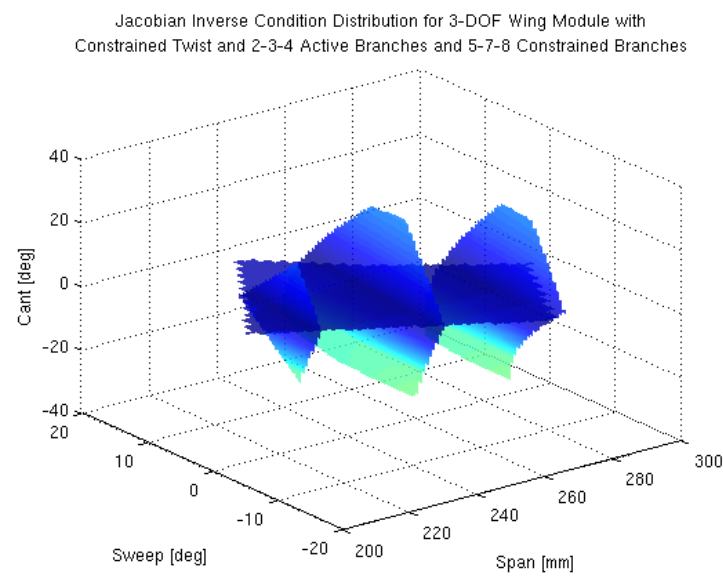

(d) 2-3-4 Active Branches

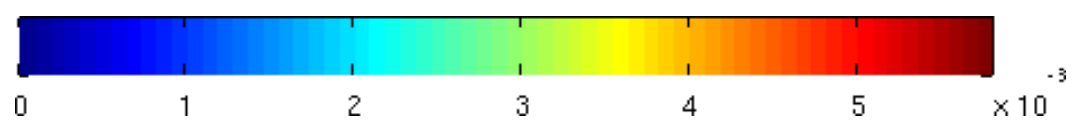

Figure 5.19: Constrained Jacobian inverse condition distribution for 2-DOF wing modules with constrained platform twist and 5-7-8 constrained branches

cant region in where the Jacobian condition is distinctly higher than the remaining workspace, a phenomenon also present of the associative 5-6-7 constraint branch configuration (see Fig. 5.17(b)). Once again though, the 1-3-4 configuration tends to perform best over the entire workspace, with a very well conditioned region within the negative cant and positive sweep region.

The 5-7-8 constraint branch configurations shown in Fig. 5.19 continue to not only show that the Jacobian condition is highly dependent on the module cant, but also that the 1-2-4 (Fig. 5.19(b)) and 1-3-4 (Fig. 5.19(c)) configurations tend to perform better than the 1-2-3 (Fig. 5.19(a)) and 2- 


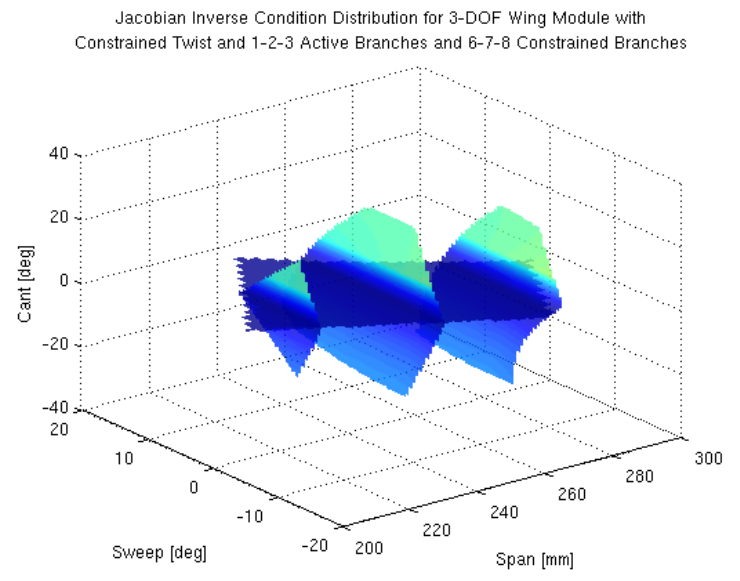

(a) 1-2-3 Active Branches

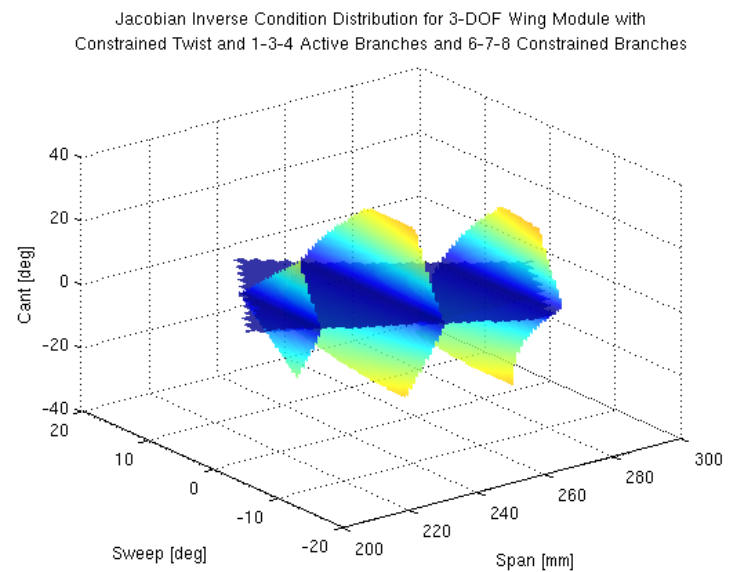

(c) 1-3-4 Active Branches

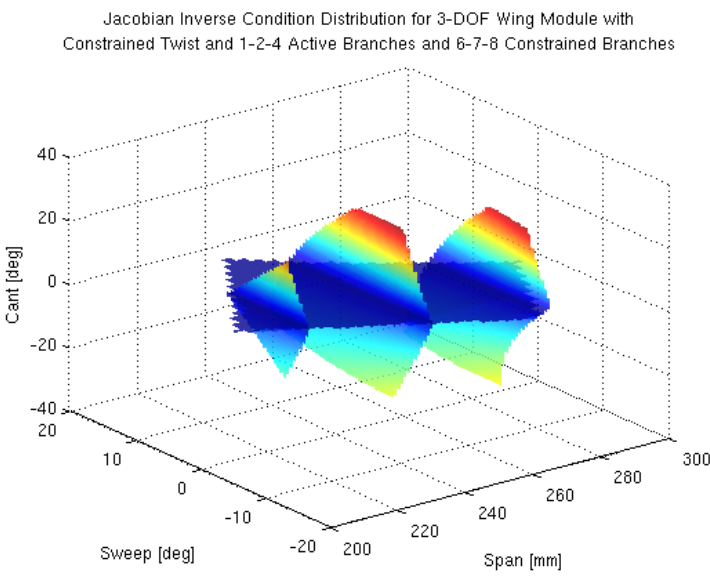

(b) 1-2-4 Active Branches

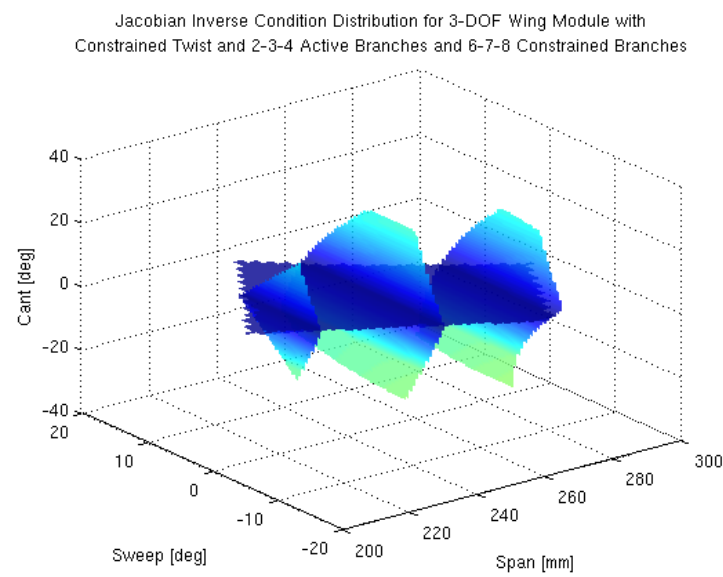

(d) 2-3-4 Active Branches

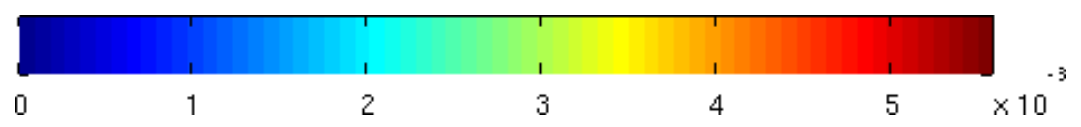

Figure 5.20: Constrained Jacobian inverse condition distribution for 2-DOF wing modules with constrained platform twist and 6-7-8 constrained branches

3-4 (Fig. 5.19(d)) configurations. First, the cant-dependency of Jacobian condition is opposite for the 1-2-3 and 2-3-4 configurations. Here, the Jacobian is well conditioned for positive and negative cant for the 1-2-3 and 2-3-4 configurations, respectively. The 1-2-4 and 1-3-4 configurations do not exhibit this opposition of Jacobian condition where both configurations are well conditioned in both the positive and negative cant regions, with greater values achieved with a positive cant. Additionally, we see that negative sweep values tend towards a better conditioned Jacobian which is in direct contrast to the 5-6-7 and 5-6-8 configurations. 
Finally, the 6-7-8 constraint branch configurations show a very similar condition distribution as compared to the 5-7-8 configurations. In Fig. 5.20, we continue to see the dominant trend of the 1-2-4 (Fig. 5.20(b)) and 1-3-4 (Fig. 5.20(c)) configurations over the 1-2-3 (Fig. 5.20(a)) and 2-3-4 (Fig. 5.20(d)) configurations. The 1-2-3 and 2-3-4 configurations again show opposition in which better conditioning is seen with positive and negative cant, respectively. Also, the 1-2-4 and 1-3-4 configurations are well conditioned for positive cant and negative sweep.

From the above analysis and figures, we see that the selection of constraint and active branches have a profound impact on the instantaneous kinematics of the 3-DOF wing module. First, we see that the 5-6-7 and 5-6-8 constraint branches show similar condition distributions, while the 5-7-8 and 6-7-8 configurations show similar distributions. This grouping is based on the presence of two constrained branches as the trailing and leading edges, respectively. From the analysis, we see that if a positive cant and negative (aft) sweep is required, then the 5-7-8 and 6-7-8 configurations perform better than the 5-6-7 and 5-6-8 configurations. Conversely, a negative cant and positive sweep requires the opposite, thus selecting constraining branches to achieve better performance can be achieved. Secondly, we see that the selection of active branches also has an impact on the Jacobian distribution. For all constraint branch configurations, the 1-2-4 and 1-3-4 configurations performed better than the 1-2-3 and 2-3-4 configurations. Here, branches 1 and 4 coalesce to a single point on the platform airfoil, with branch 2 or 4 connected at the opposite corner of the wing truss. This configuration seems to consistently provide better overall performance with an slight edge to the 1-3-4 configuration for the 5-6-7 and 5-6-8 constraint branches, and the 1-2-4 for the 57-8 and 6-7-8 configurations. The combination of two active branches and constrained branches at the leading and trailing edges, respective result in better instantaneous kinematic performance, and thus intelligent selection of the module configuration can lead to better overall module kinematic performance.

Finally, as with the 4-DOF wing modules, singularities are present in the workspaces of each 3-DOF wing configuration. The singularities occur when both the sweep and cant are zero for all module span. In the current configuration setup, branches 1 and 3 and branches 2 and 4 oppose each 
Table 5.7: Constrained Jacobian inverse condition data for the 2-DOF wing module configurations

\begin{tabular}{llllll}
\hline Independ. & Active & \multicolumn{4}{l}{ Constrained Jacobian Inverse Condition } \\
Motion & Branches & $\boldsymbol{\mu}_{\max } \times \mathbf{1 0}^{\mathbf{- 3}}$ & $\boldsymbol{\mu}_{\min } \times \mathbf{1 0}^{-\mathbf{5}}$ & $\boldsymbol{\mu}_{\text {avg }} \times \mathbf{1 0}^{-\mathbf{4}}$ & $\boldsymbol{\mu}_{\text {std }} \times \mathbf{1 0}^{-\mathbf{4}}$ \\
\hline$\Lambda$ & $1-2$ & 8.0958 & 374.69 & 57.02 & 14.225 \\
& $1-3$ & 6.385 & 440.05 & 55.222 & 8.7474 \\
& $1-4$ & 6.0539 & 0.11768 & 17.248 & 17.55 \\
& $2-3$ & 0.60471 & $9.3428 \times 10^{-3}$ & 2.0749 & 1.4929 \\
& $2-4$ & 7.9198 & 364.07 & 56.527 & 13.838 \\
& $3-4$ & 6.371 & 440.33 & 54.881 & 8.3742 \\
& & & & \\
& $1-2$ & 19.998 & 366.6 & 61.171 & 38.558 \\
& $1-3$ & 20.0 & 440.0 & 66.237 & 37.425 \\
& $1-4$ & 6.1471 & 0.26463 & 18.361 & 18.64 \\
& $2-3$ & 0.78159 & 0.18908 & 2.316 & 1.7847 \\
& $2-4$ & 20.001 & 356.11 & 61.615 & 38.548 \\
& $3-4$ & 20.004 & 440.27 & 66.882 & 37.413 \\
& & & & \\
& $1-2$ & 1.1776 & 0.049271 & 4.3048 & 3.8282 \\
& $1-3$ & 1.5486 & 0.04928 & 5.2036 & 4.7371 \\
& $1-4$ & 1.4132 & $7.6894 \times 10^{-6}$ & 3.3401 & 4.1106 \\
& $2-3$ & 0.17693 & $2.861 \times 10^{-6}$ & 0.42035 & 0.44583 \\
& $2-4$ & 1.2275 & 0.049272 & 4.392 & 3.9465 \\
& $3-4$ & 1.6215 & 0.04928 & 5.337 & 4.9039 \\
\hline
\end{tabular}

other in direction, thus when there is no platform airfoil rotation, there are always two branches which are directed opposite to each other, which reduces the rank of the constrained Jacobian. This in turn leads to singularities within the workspace.

\subsubsection{2-DOF Jacobian Inverse Condition Distribution}

The 2-DOF wing configurations require all four of the axial branches to be constrained, and two of the four diagonal branches to be active. This leads to six unique configurations for each independent platform rotations.

The collected data in Table 5.7 shows that there is a distinct disadvantage for the 1-4 and 2-3 active branch configurations with respect to the constrained Jacobian inverse condition. For each independent platform motion, the 1-2, 1-3, 2-4 and 3-4 active branch configurations outperforms the 1-4 and 2-3 configurations, however, the 2-3 configuration exhibits the poorest performance. This is particularly true for the non-singular minimum condition values which are significantly 
smaller than those of the other active branch combinations. The 1-4 and 2-3 configurations each have both active branches coalescing to a single point, thus restricting the ability for the prismatic actuators to push the platform airfoil. The remaining configurations each consists of an active branch connected at the leading and trailing edge of the wing module. This separation of active branches clearly demonstrates better instantaneous kinematic behaviour, and thus the 1-2, 1-3, 2-4 and 3-4 configurations are favoured.

The inverse condition distribution of the constrained Jacobian is shown in Fig. 5.21 for the sweep-independent wing modules. The 1-4 and 2-3 modules shown in Fig. 5.21(c) and Fig. 5.21(d), respectively, show that the condition distribution is poor compared to the other configurations. The 1-2 (Fig. 5.21(a)), 1-3 (Fig. 5.21(b)), 2-4 (Fig. 5.21(e)), and 3-4 (Fig. 5.21(f)) modules each shown a better distribution, albeit distributions that are sided in favor of negative sweep values. This is, in general, a positive feature as negative sweep indicates an aft sweep motion, which would be required more than a forward sweep motion. We do note that for the 1-2, 1-3, 2-4 and 3-4 configurations, there is a discontinuous transition in the condition distribution between positive and negative sweep values. Each sign of sweep displays almost uniform condition distribution and with little condition variation with respect to the module span, and we see that for these configurations, the condition is dependent only on the sign of the module sweep, with the obvious exception being the 1-4 active branch configuration. The best distribution is observed with the 2-4 configuration.

The inverse constrained Jacobian condition distribution for the cant-independent wing modules are shown in Fig. 5.22. Similarly to the sweep-independent modules, the Jacobian condition of the cant-independent modules shows little dependence on the span of the module, but is highly dependent on the module cant. Once again the 1-4 (Fig. 5.22(c)) and 2-3 (Fig. 5.22(d)) active branch modules exhibit a poor condition distribution compared to the remaining configurations. For the remaining configurations, the condition distribution is sided depending on the sign of the module cant, with slightly more favourable condition when the module is in a negative cant position. The 1-2 (Fig. 5.22(a)), 1-3 (Fig. 5.22(b)), 2-4 (Fig. 5.22(e)) and 3-4 (Fig. 5.22(f)) configurations all show high conditioning throughout the workspace. This is particularly true when the module cant 


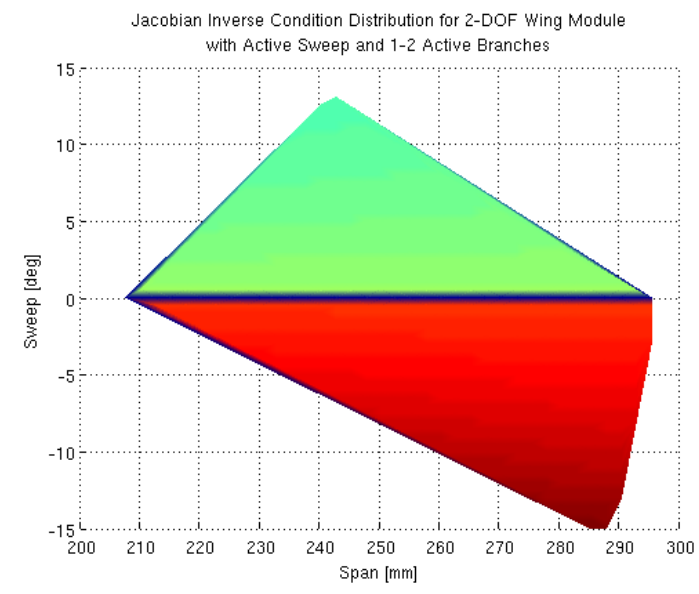

(a) 1-2 Active Branches

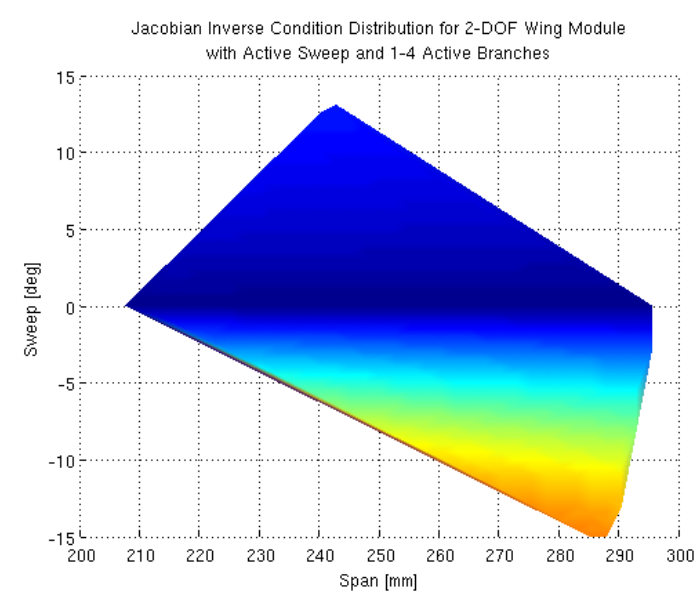

(c) 1-4 Active Branches

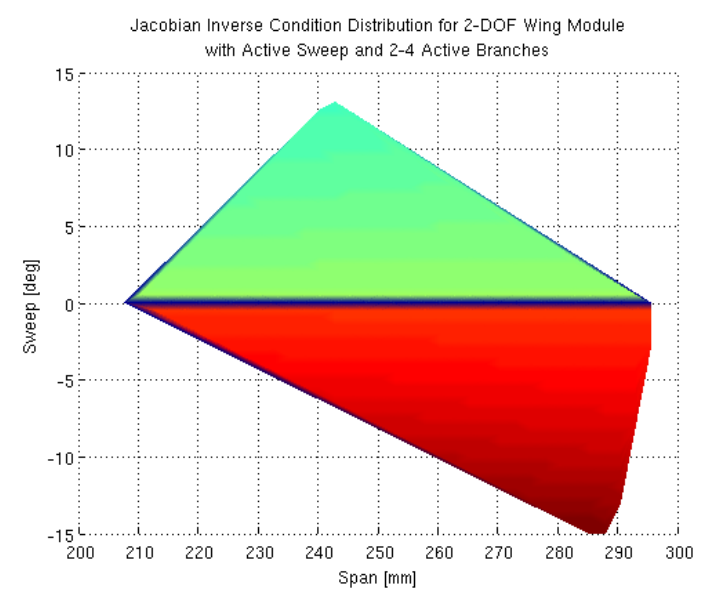

(e) 2-4 Active Branches

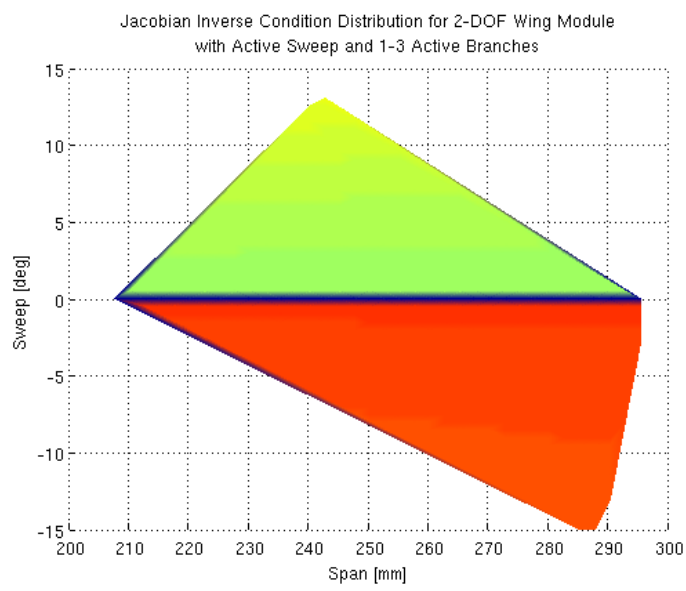

(b) 1-3 Active Branches

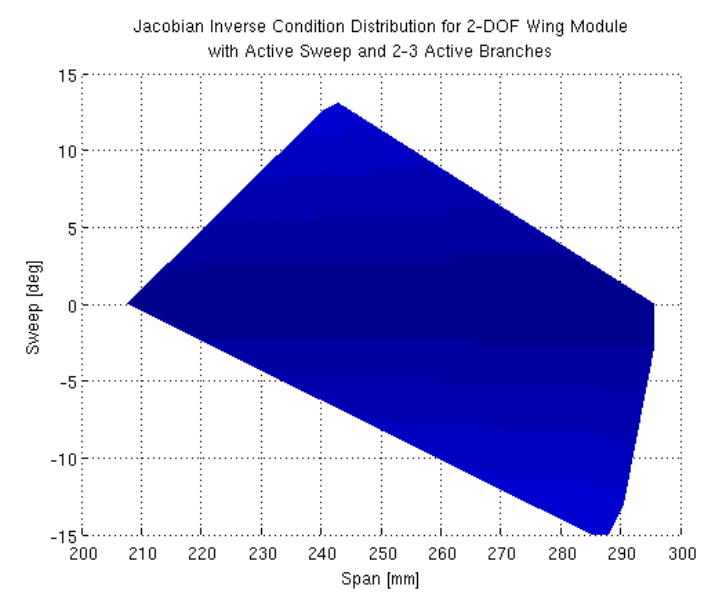

(d) 2-3 Active Branches

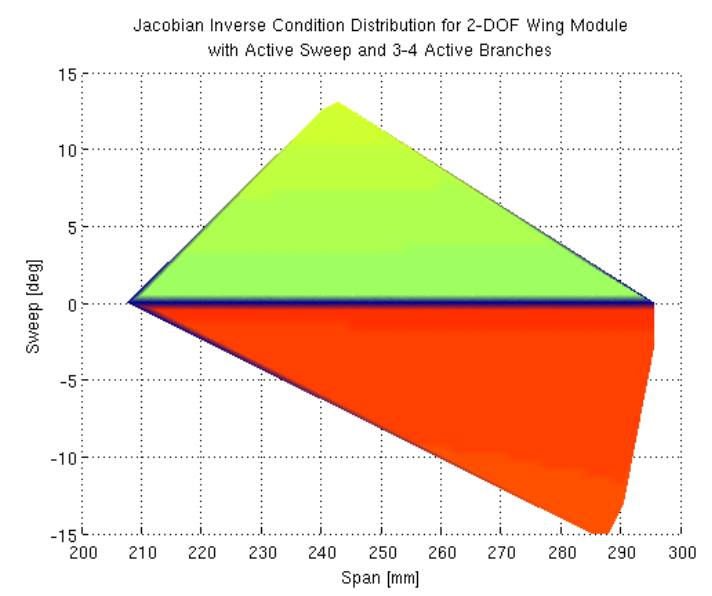

(f) 3-4 Active Branches

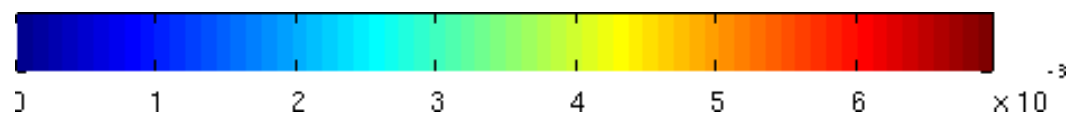

Figure 5.21: Constrained Jacobian inverse condition distribution for 2-DOF wing modules with active platform sweep 


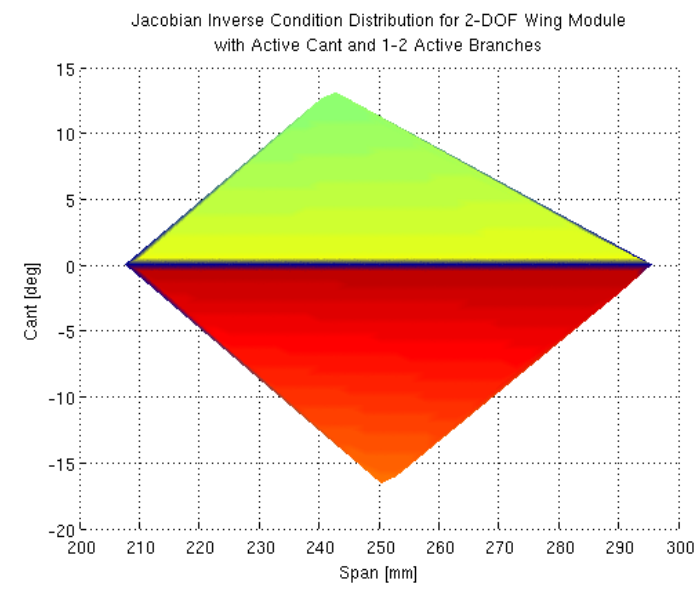

(a) 1-2 Active Branches

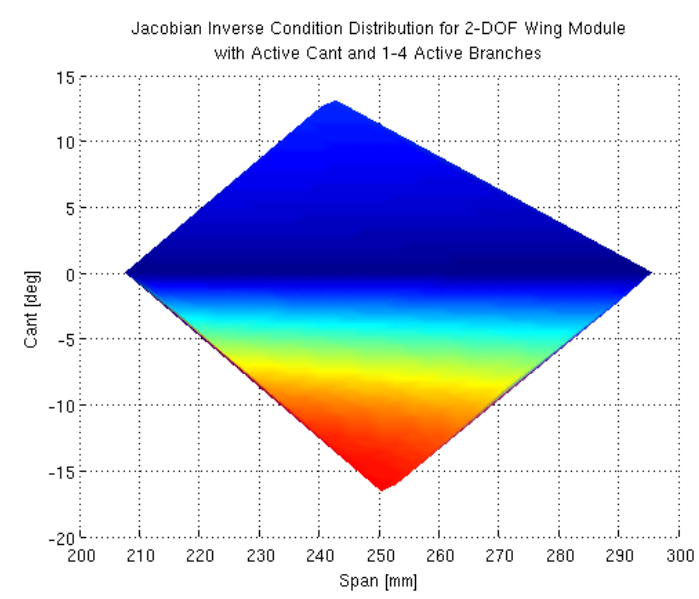

(c) 1-4 Active Branches

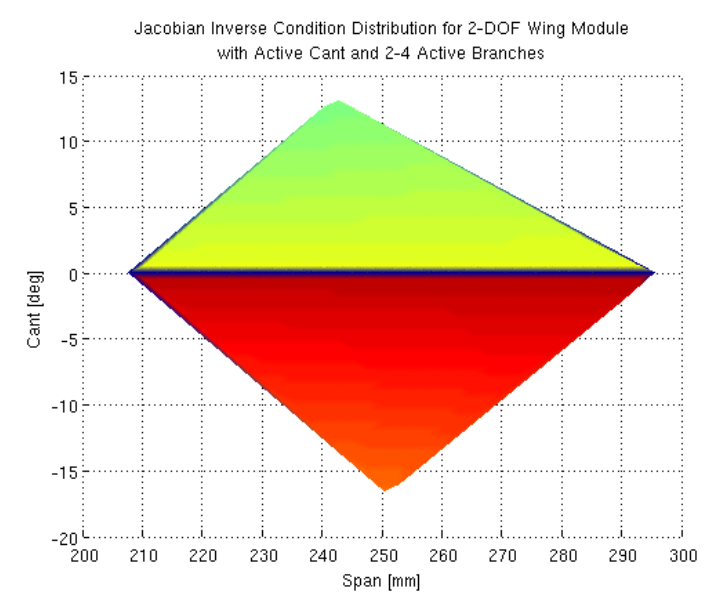

(e) 2-4 Active Branches

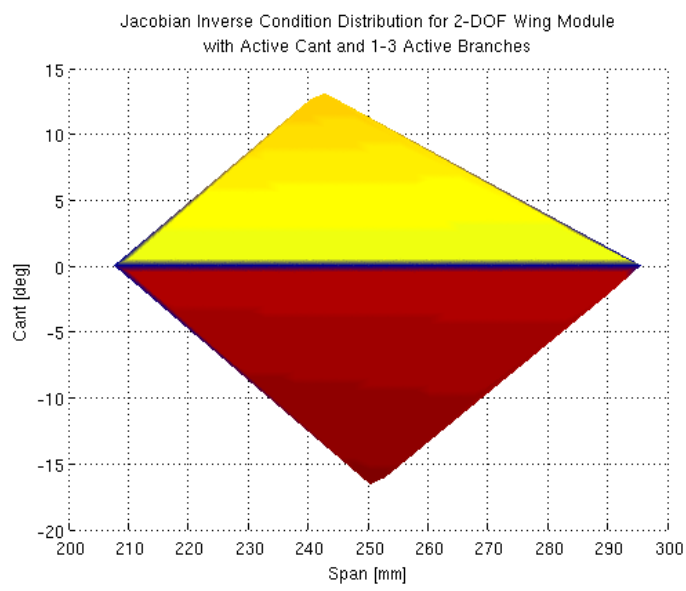

(b) 1-3 Active Branches

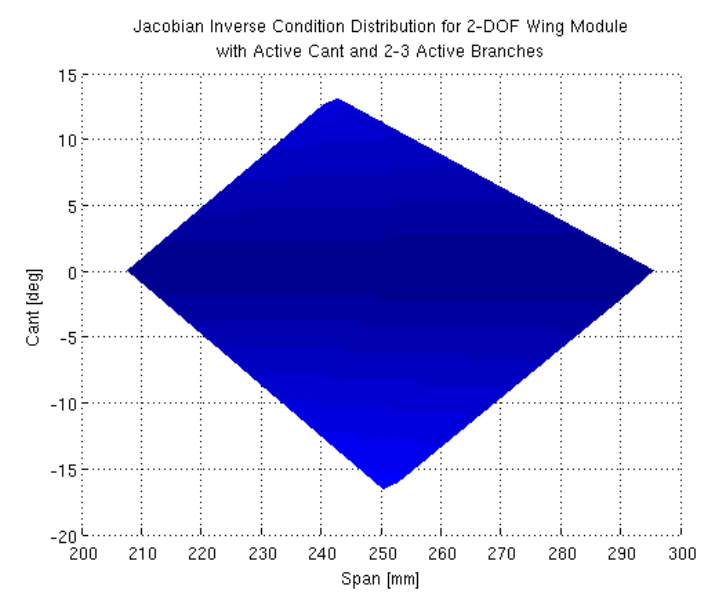

(d) 2-3 Active Branches

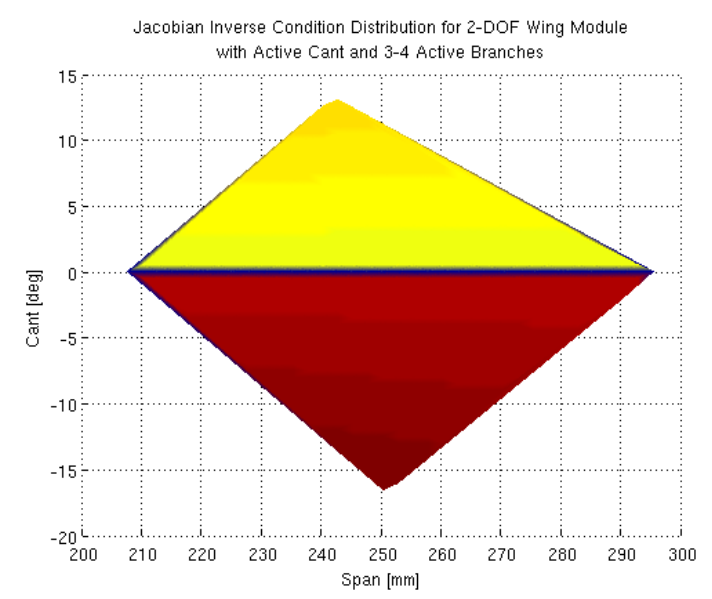

(f) 3-4 Active Branches

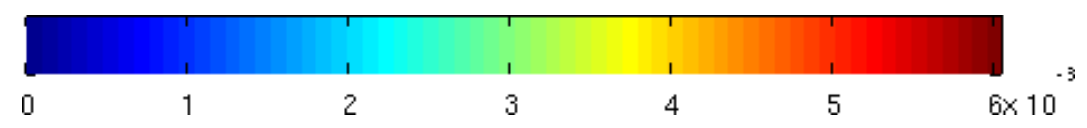

Figure 5.22: Constrained Jacobian inverse condition distribution for 2-DOF wing modules with active platform cant 


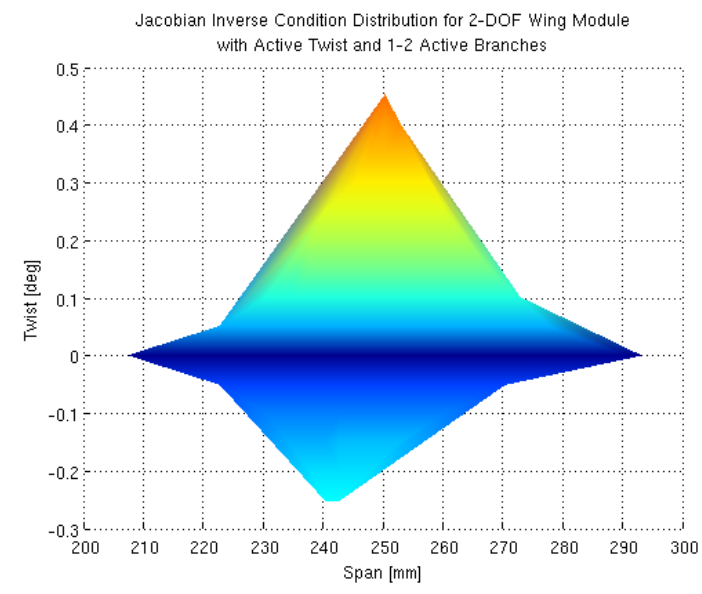

(a) 1-2 Active Branches

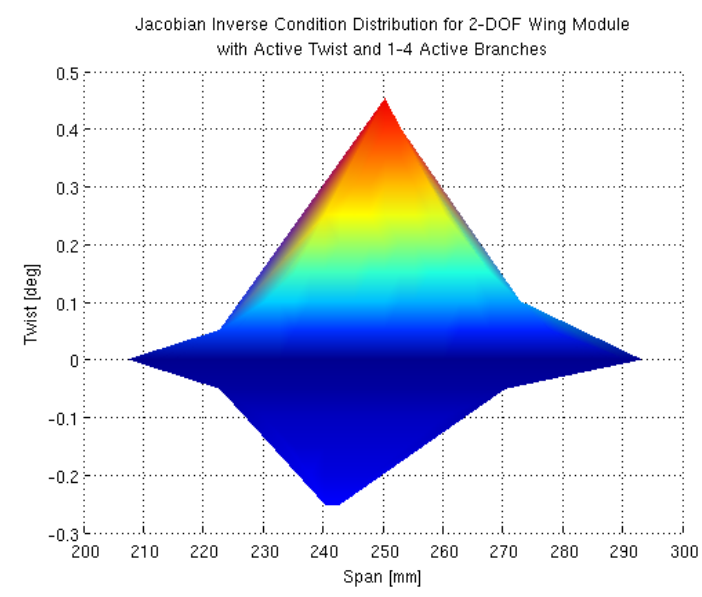

(c) 1-4 Active Branches

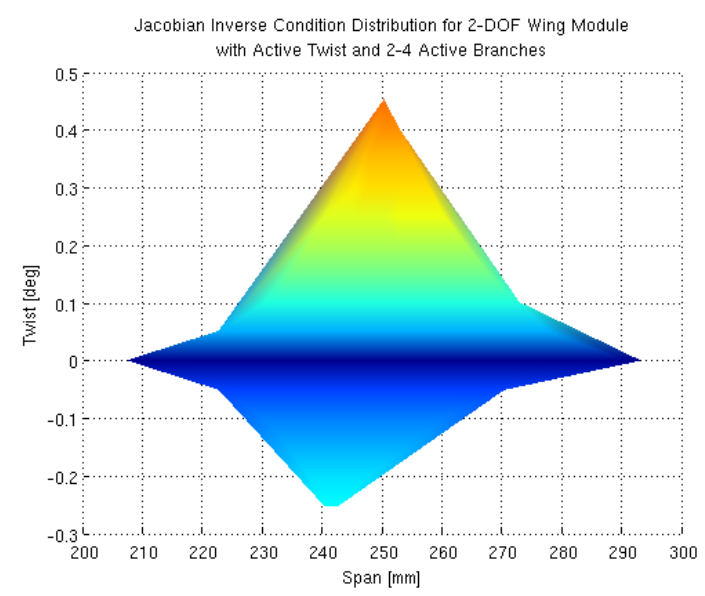

(e) 2-4 Active Branches

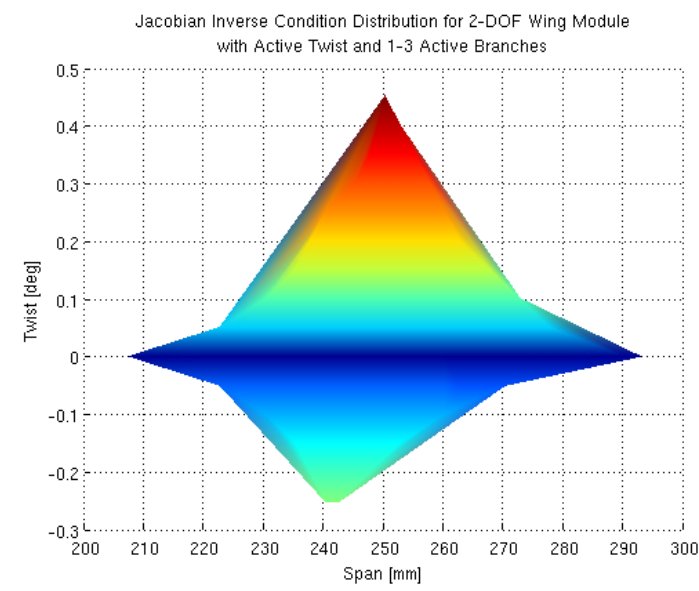

(b) 1-3 Active Branches

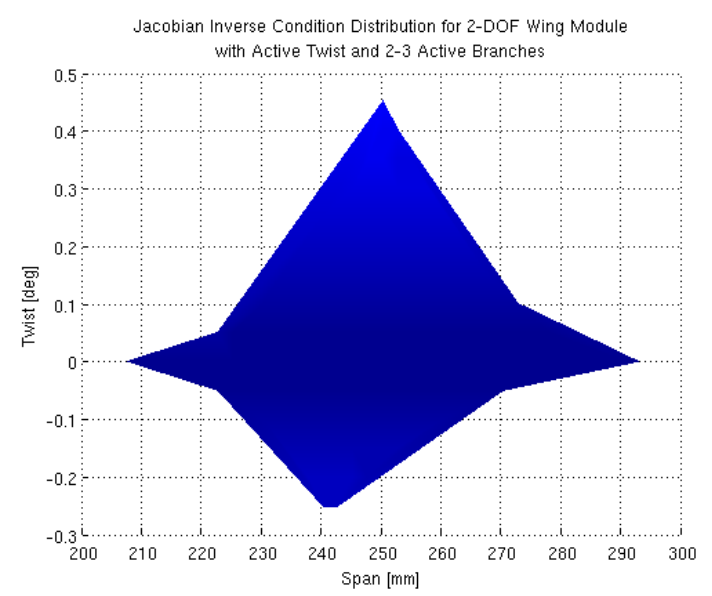

(d) 2-3 Active Branches

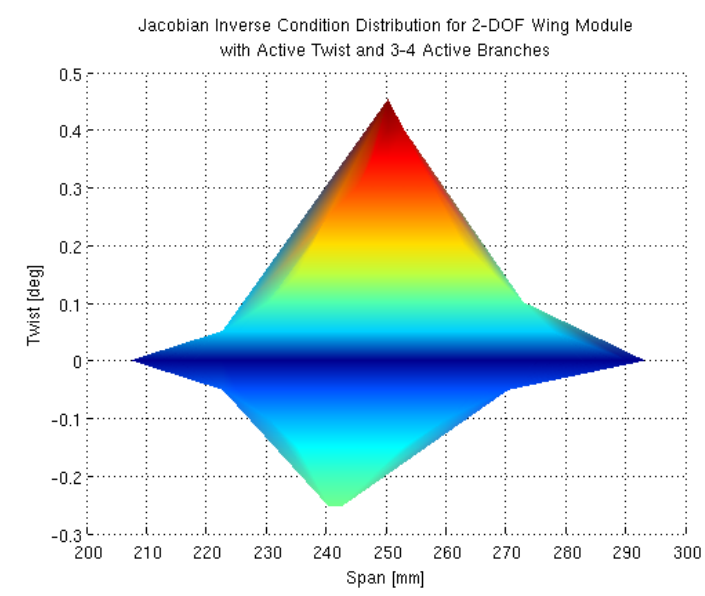

(f) 3-4 Active Branches

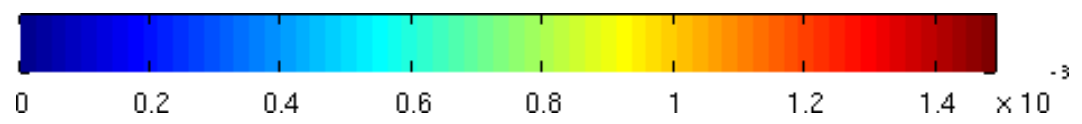

Figure 5.23: Constrained Jacobian inverse condition distribution for 2-DOF wing modules with active platform twist 
close to, but not equal to zero, where we can see the condition number is the highest. However much like the sweep-independent configurations, there is a discontinuity in Jacobian condition while transitioning from positive to negative cant values. Here, the 3-4 configuration is best suited with respect to Jacobian conditioning.

Finally, the constrained Jacobian inverse condition distribution for the 2-DOF sweep-independent wing modules is shown in Fig. 5.23. Much like the sweep and cant-independent modules, the 1-4 and 2-3 active branch configurations, shown in Fig. 5.23(c) and Fig. 5.23(d), respectively, shown the poorest condition performance, with the 2-3 configuration continuity to exhibit the worst condition distribution. Here though, there is a gradual transition in condition as the module twist is increased or decrease, with very little dependency on the span of the module. We see that for the 1-4, as well as the 1-2 (Fig. 5.23(a)), 1-3 (Fig. 5.23(b)), 2-4 (Fig. 5.23(e)) and 3-4 (Fig. 5.23(f)) modules, the highest condition is achieved at the maximum module twist. Although not as high as positive twist values, the condition also increases as the twist decreases and reaches the minimum value. This is opposite to what was shown with the sweep and cant-independent modules in which the highest condition is experienced when there the sweep and cant were both close to, but not equal to zero. The best performance however, which is also shown in Table 5.7, are the 1-3 and 3-4 active branch configurations.

With respect to singularities in the workspace, the 2-DOF wing modules are not singularity free. Much like the 4-DOF and 3-DOF wing modules, singularities are present when the platform airfoil is parallel to the base airfoil, i.e., no module sweep, cant or twist.

\subsubsection{Kinematics Summary}

In this section, the kinematics and instantaneous kinematics of the wing module configurations were explored in detail. In Section 5.3.1, the kinematic range of platform variables was analyzed with the expressed goal of determining which constraint branch combination provided the best kinematic performance for the 4-DOF and 3-DOF wing modules. The 2-DOF wing module posses four constrained branches and thus only one configuration is possible. From the analysis, it was 
evident that the combination of constrained branches has a profound impact on the kinematic performance of the wing with respect to the module twist, whereas the kinematic performance of the sweep and cant was relatively independent of constraint branch combination. This is due to the constraint plane orientations being perpendicular to the base airfoil and passing through the base airfoil $z$-axis. As such, the planar motion of the spherical joints on the constrained branches tends in the direction required for sweep or cant motion of the platform airfoil. Twist motion on the other hand is perpendicular to the constraint planes and is thus limiting depending on which branches are constrained. As was shown, diagonally opposed constrained branches are the most limiting combination, which is avoidable for the 4-DOF configurations, but unavoidable for the 3-DOF and 2-DOF configurations. Despite this being a limiting factor, a decent twist range is achievable for the 3-DOF configurations.

The instantaneous kinematics of the wing module were analyzed in Section 5.3.2 where the constrained Jacobian inverse condition distribution was used as a performance parameter of the wing modules. Where the kinematics of the modules are dependent only on the constraint branch combination, the instantaneous kinematics are dependent on both the active and constraint branch combinations, leading to further opportunity for reconfiguration, and further configurations for analysis, especially for the 3-DOF wing modules. Consistently, the Jacobian condition improved as the platform airfoil was actuated away from a position in which the platform airfoil was parallel to the base airfoil. With no platform airfoil rotation, singularities are present, requiring additional measure to be taken when instantaneous motion is to be used. However, better Jacobian condition is experienced as the platform airfoil is reoriented away from the original parallel position. This is consistent for parallel robots with this type of constraint system [59,64].

The kinematic analysis shown was for specific constraint branch orientations. Specifically, constraint planes were restricted to be perpendicular to the base airfoil and pass through the $z_{b}$-axis as is a common setup for traditional constrained parallel robots. This setup is not necessarily a requirement, and any constraint plane orientation can theoretically be used without having to reformulate the kinematic equations. As such, there is an opportunity to optimize the kinematic 
performance of the module by altering the orientations of the constraint planes. This is, however. beyond the scope of this these.

Finally, what was shown from the kinematic analysis was that the range of performance values can be used to select the best suited module, on-demand. The module configuration can be selected based not only on the kinematic variable range, but also the instantaneous kinematic performance. This unique feature allows an extremely high level of flexibility for the wing module.

\subsection{Summary}

In this chapter, the kinematics of the wing module were evaluated. First, a new parametric constraint kinematic formulation was developed for the inverse and instantaneous kinematics of the wing module. The developed parametric formulation is more versatile than previous constraint systems and can be applied to other parallel robot systems. To evaluate the kinematic potential of each wing module mobility, the constrained inverse and instantaneous kinematics were computed and analyzed. Platform airfoil variable ranges were determined as well as the constrained Jacobian inverse condition distribution. Computational comparisons highlight performance capabilities and limitations of the module configurations, which can be used for optimal configuration selection. The benefits of the proposed constraint system were shown, as internal reconfiguration of the wing module leads to more desirable kinematic performance for a given mobility requirement. 


\section{Chapter 6}

\section{Wing Module Prototype Development}

In this chapter, a discussion on the development of the wing module prototype is given. First, a brief description of the wing module is given including a description of the active and passive branch components. With the wing module described, the kinematic response of the wing is shown as it pertains to proving the developed kinematics. This is followed by a discussion on morphing skin systems including the development of the morphing skin system using the developed module kinematics. Finally, conclusions are given.

\subsection{Module Prototype}

In this section, a description of the module prototype is given. First, a physical description of the module is given including descriptions of components used. This is followed by the kinematic response of the wing module.

\subsubsection{Module System Description}

One of the major features of the developed morphing wing system is modularity. The benefits of modularity as previously mentioned is that analysis of a full system can be reduced to that for a single module. In previous chapters, this feature has been exploited for the architecture, mobility and kinematics analyses. This feature is further exploited for the development of a prototype, where a single controllable wing module was developed with the goal of validating the developed mobility and kinematic formulations. 


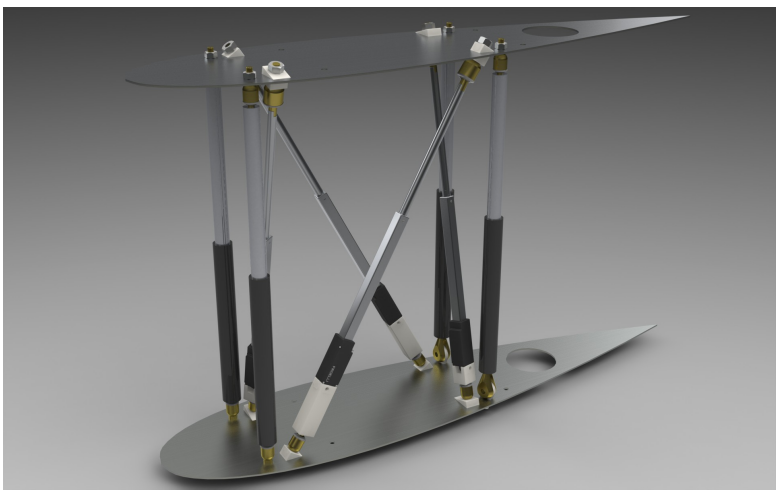

(a) Prototype solid model

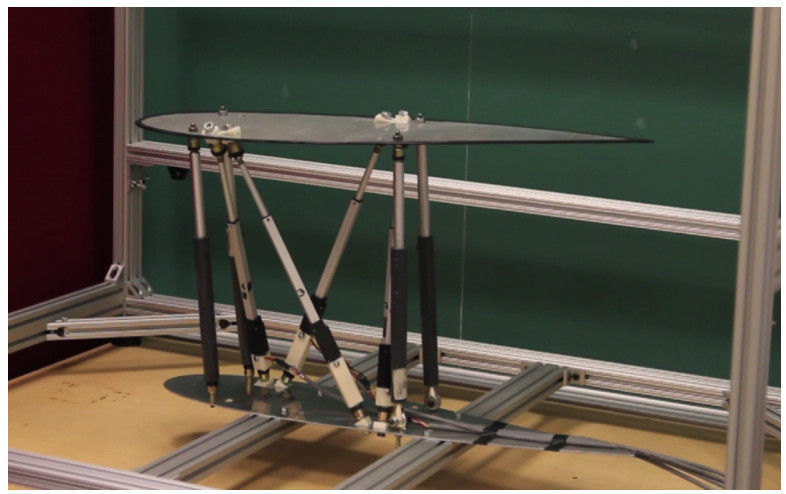

(b) Wing module prototype

Figure 6.1: Mechanical prototype of wing module

The 4-DOF module with 5-6 constraint branches was used for the wing module prototype which is shown in Fig. 6.1. This configuration was selected based on the resultant kinematic capabilities analyzed in Chapter 5. However, the prototype can be reconfigured to any other 4-DOF configuration by swapping the constrained branches accordingly. The solid model in Fig. 6.1(a) depicts an early design configuration of the wing module in which the active branches are diagonally positioned following the same direction, as opposed to opposing directions for upper/lower and leading/trailing branches shown in Fig. 6.1. The branches of the prototype consist of four unconstrained-active, two unconstrained-passive and two constrained-passive branches. The two constrained-passive branches are configured to the branch architecture outlined in Section 4.2.2.2 and have $\mathrm{R}_{\mathrm{T}} \mathrm{P}_{\mathrm{V}} \mathrm{S}$ joint architecture. The unconstrained branches are however configured with $\mathrm{SP}_{\mathrm{V}} \mathrm{S}$ joint architecture in lieu of a UPV $\mathrm{S}$ branch. The additional degree-of-freedom replaces the universal joint on the base airfoil with a spherical joint, raising the connectivity of the branch to $C_{k}=7$. This renders the branch redundant by a single degree-of-freedom since $\left(C_{k}=7\right)>(\lambda=6)$. The reason for the unconstrained branches to be configured as such was for strength considerations. If an unconstrained branch connects to the base and platform airfoil ribs with a spherical joint, or plain spherical bearing, then moments are not transferred to the branch, thus reducing the strength requirement of the branch. The unavoidable result of this configuration is the alignment of two rotation axes, one from each spherical joint, which align along the branch vector, $\mathbf{d}_{k}$. This allows the prismatic joint to rotate axially, or spin, about this axis. Although kinematically undesirable and unnecessary, very little 
axial rotation of the prismatic joint was observed and did not negatively affect the mobility of the wing system.

To provide controllable motion, actuators from Firgelli Technologies, Inc, were selected based on their compact size and their performance characteristics. The L16-P 150:1 $140 \mathrm{~mm}$ actuator was used with technical specs outlined in Table 6.1. A compact actuator is essential due to volume limitations within the wing module. A 150:1 gear reduction ratio was selected which limited the maximum speed of the actuator to $8 \mathrm{~mm} / \mathrm{s}$ under no load. A slow morphing maneuver is required to prevent quick changes in aerodynamic forces, thus the low actuation speed is beneficial. The 150:1 gear reduction ratio provides the maximum lifting capabilities of the actuator while minimizing the weight of the actuator-another important requirement. Despite the compact size, the actuator can provide $200 \mathrm{~N}$ lift force and $102 \mathrm{~N}$ back drive force. Additionally, the ability to withstand side forces is essential due to the direction of lift and drag forces with respect to the actuator orientation. The large electrical stroke of $138 \mathrm{~mm}$ allows for significant workspace to be reached. Finally, suitable positional accuracy and mechanical backlash allows for precise repeatability.

The prismatic joints of the passive branches are required to react to the kinematic motion of the platform, with an ability to lock when not in motion. Locking the extension capabilities of the passive branches maintains the structural integrity of the truss when the wing is not transitioning from one position to another. The passive prismatic joints are a piston-cylinder style joint with PVC tubing used for the cylinder and a turned aluminum rod used for the cylinder. For compatibility with the Firgelli actuators, the passive branches were designed to achieve a maximum stroke of 138

mm. A set screw was threaded into the side of each PVC tube and tightened to lock the passive branches when not in motion.

\subsubsection{Kinematic Response}

The kinematic response of the wing module is used to prove the developed kinematic formulations. In order to control the wing module, a hardware-software integrated control system was developed using Matlab Simulink, and custom controller developed by Quanser. The control hardware from 
Table 6.1: Firgelli Technologies, Inc L16 150:1 140 mm actuator characteristics [75]

\begin{tabular}{ll}
\hline Characteristic & Value \\
\hline Gear reduction & $150: 1$ \\
Peak power & $75 \mathrm{~N}$ at $7 \mathrm{~mm} / \mathrm{s}$ \\
Max speed (no load) & $8 \mathrm{~mm} / \mathrm{s}$ \\
Max force (lifted) & $200 \mathrm{~N}$ \\
Back drive force & $102 \mathrm{~N}$ \\
Max side load (extended) & $20 \mathrm{~N}$ \\
Mass & $84 \mathrm{~g}$ \\
Electrical stroke & $138 \mathrm{~mm}$ \\
Positional accuracy & $0.5 \mathrm{~mm}$ \\
Mechanical backlash & $0.2 \mathrm{~mm}$ \\
\hline
\end{tabular}

Quanser interfaces with Matlab Simulink through QUARC, an interface software. Specialized Simulink blocks enable standard Simulink signals to be read by QUARC, transfer the signals to the controller which in turn drives the actuators within the wing module system.

Figure 6.1.2 shows a custom interface developed in Matlab Guide. The interface is the front end of the control system in which user-specific wing module sweep, cant, twist and span inputs are used to actuate the wing module. All four wing degrees-of-freedom must be entered into the control system. The prototype kinematics are identical to those developed in Chapter 5. Individual morphing motions are shown in Fig. 6.3. Here, individual sweep (Fig. 6.3(a)), cant (Fig. 6.3(b)) and twist (Fig. 6.3(c)) motions are shown at a spcific span. For each of the above platform rotations, the other platform rotations were specified to be 0. Fig. $6.3(\mathrm{~d})$ shows the prototype with a specific span, and no rotations. A more complicated morphing maneuver is shown in Fig. 6.1.2 where a combination of sweep, cant, twist and span were input, and the resultant motion of the platform airfoil was observed. The full video is available in Appendix C. 


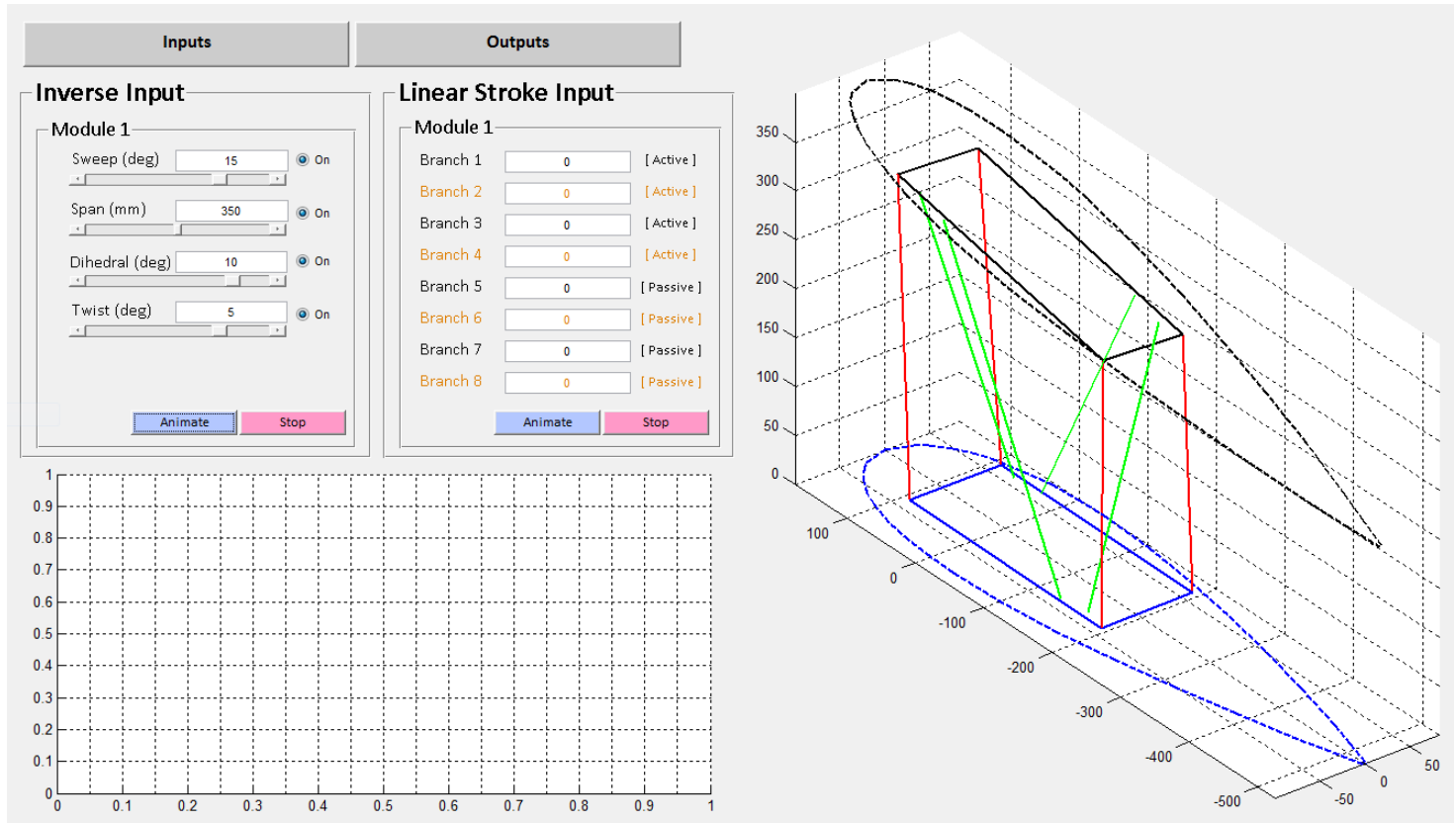

Figure 6.2: Screen shot of wing module prototype control interface

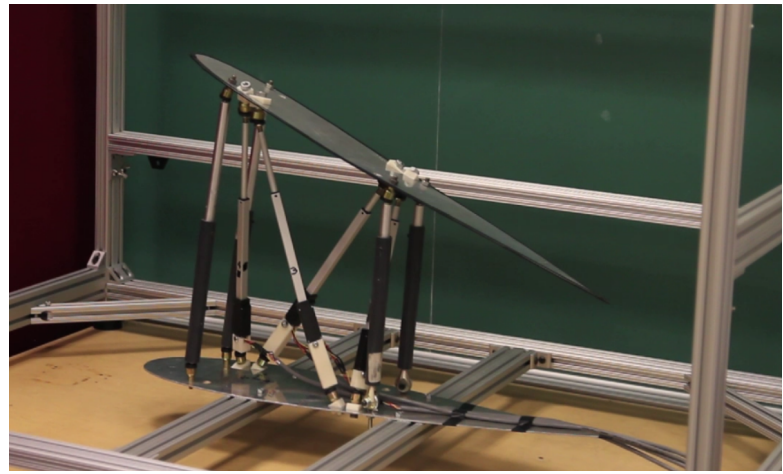

(a) Sweep

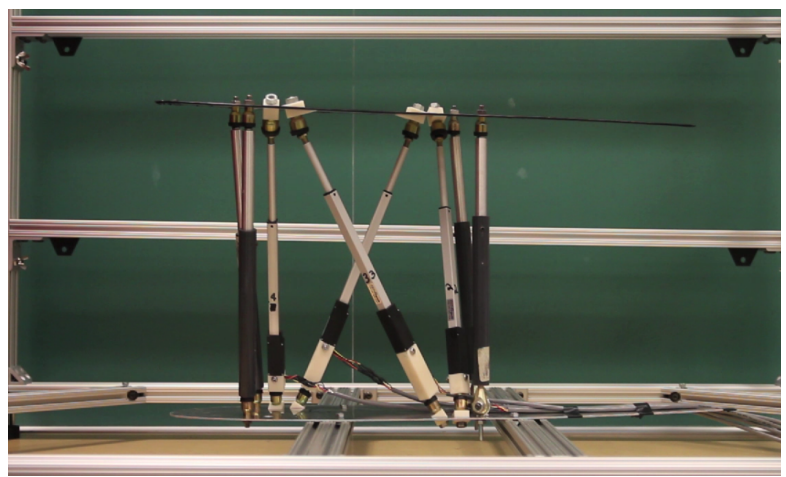

(c) Twist

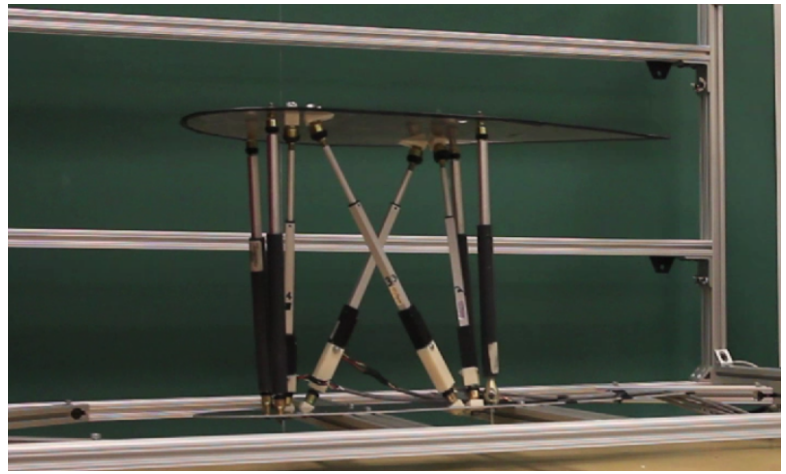

(b) Cant

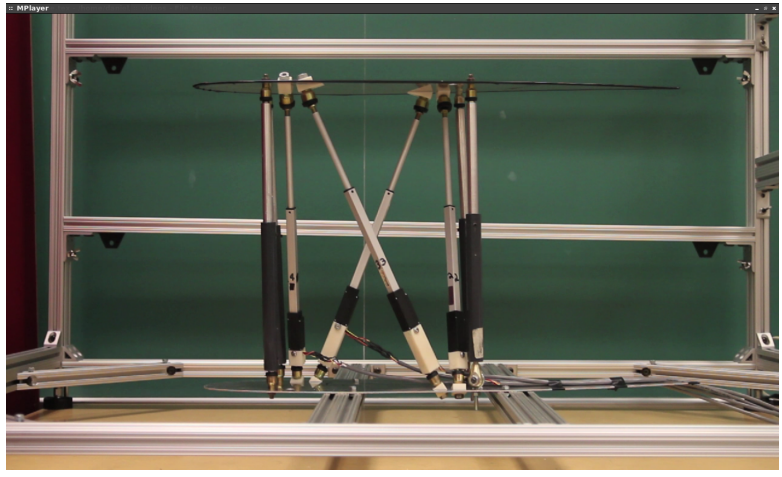

(d) Span

Figure 6.3: Wing module prototype with single morphing degree-of-freedom pose 


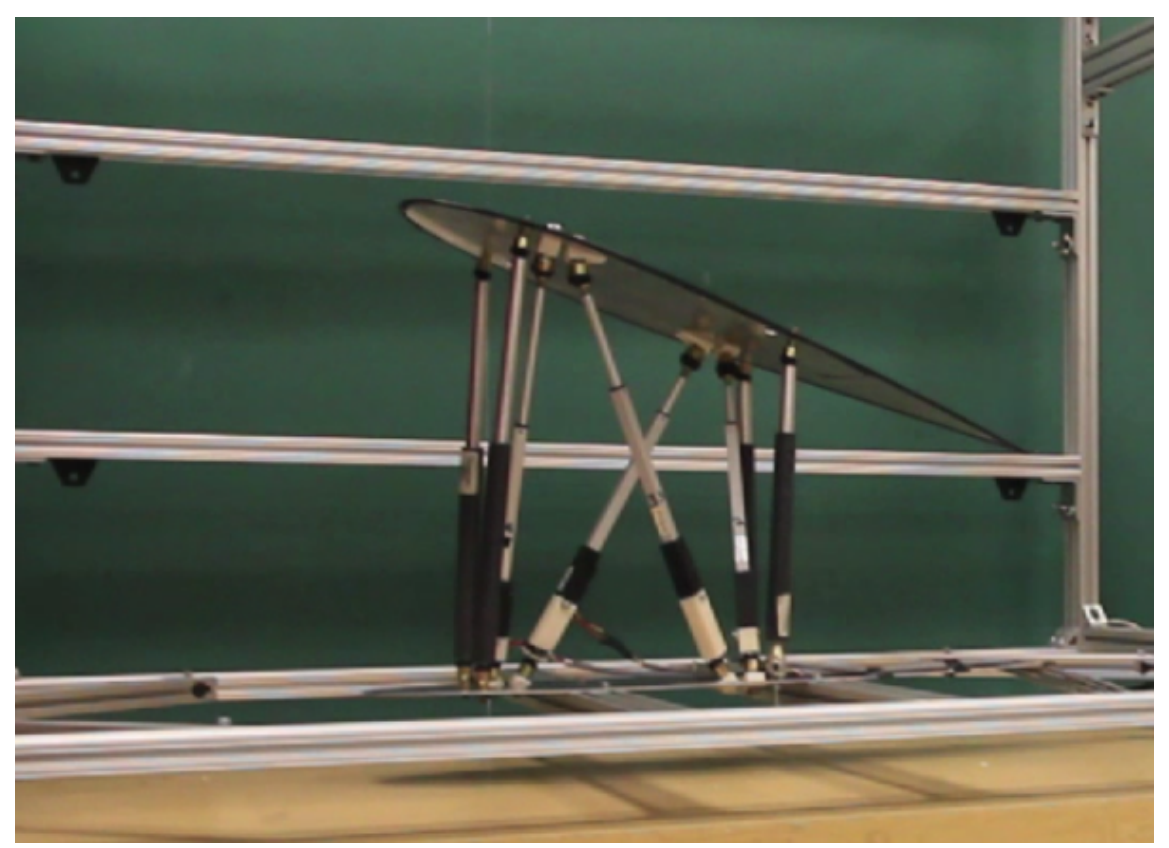

Figure 6.4: Wing module prototype with multiple degree-of-freedom pose, utilizing 4 degrees-offreedom

\subsection{Wing Module Skin System Conceptual Design}

One particularly challenging aspect associated with wing morphing is the implementation of morphing skins systems. At the highest level, a skin system for a morphing wing must maintain an adequate aerodynamic surface, while simultaneously supporting the associative loads experienced during flight. While many wing morphing projects have been proposed, as surveyed in Chapter 2, the skin systems utilized are equally as diverse as the projects to which they are associated. However, as with the mechanisms to which they are paired, the developed morphing skin systems are suitable only for a single type of morphing. For the proposed modular morphing wing, a more versatile skin system is required as the module is expected to assume varying motion profiles.

The detailed development of a skin system is beyond the scope of this dissertation as is a survey of current developments in morphing wing skin systems. However, the high level kinematic requirements of a skin system can be formulated and identified based on the predicted motion of the wing module. The goal here is to observe how the the skin would change with respect to morphing. In this section, an overview of potential skin systems is given for the wing module, followed by a 
kinematic analysis for the conceptual design of a skin system for the wing module.

\subsubsection{Morphing Wing Skin Systems}

For an individual morphing degree-of-freedom, a unique morphing skin system can be developed once the expected motion range is established. The challenge however for the morphing wing module is that the skin system is expected to satisfy a variety of motion requirements that are not necessarily uniform over the entire wing module skin surface. Each morphing-degree-of-freedom imposes drastically different requirements and resultant wing skin motion profiles. For example, span morphing requires the skin to expand and retract uniformly, however sweep motion requires non-uniform skin expansion and retraction. Inoyama et al. show in their work the variation in skin system requirements for their planar morphing wing $[42,43]$. For the morphing wing module, addressing the unique skin motion requirements for each individual morphing degree-of-freedom would not suffice, as the wing skin must be able to satisfy a combination of motion requirements. As such, an analysis on the kinematic response of the wing skin must be performed.

Categorically, a morphing wing skin can be $a$ ) a system of sliding rigid bodies, $b$ ) a stretchable material wrapped around the wing, or $c$ ) a hybrid system combing elements of sliding and stretchable skins.Each morphing wing skin system is briefly described with respect to the four wing module degrees-of-freedom.

\subsubsection{Sliding Skin System}

A sliding skin system consists of one or more rigid bodies that slides against each other and/or static wing surfaces during a morphing maneuver. This method is popular with some sweepmorphing and span-morphing wings where rigid wing sections slide within an adjacent hollow wing or body $[11,29,30,34,35]$. As such, two rigid surfaces remain in contact and slide against each other during morphing. An alternative method that has been proposed for sweep motion is a series of rigid 'feathers' that rotate about the sweep rotation axis [15]. The use of sliding skins for sweep and span morphing can be attributed to the fact that both motions are planar, and the sliding skin surfaces are easier to implement. 
Benefits of sliding skins are attributed to the fact that the rigid bodies provide a suitable surface to withstand aerodynamic forces, particularly the pressure distribution. Penalties include the additional weight of overlapping skin sections, with major challenges of maintaining a seal between two adjacent skin surfaces to prevent air flow within the wing volume. Despite these challenges, a sliding skin system has potential to be used in a poly-morphing wing system.

For the wing module, the combined platform rotations requires that any sliding skin remains in contact with an adjacent surfaces that are not necessarily planar for a given morphing maneuver; i.e. the local orientation between the sliding skin and adjacent surface is not consistent during the full morphing motion. The in-plane sweep and span motion, combined with the out-of-plane cant and twist motion results in skin surfaces that must posses multiple degrees-of-freedom in order to maintain contact with adjacent surfaces. Additionally, the curvature variation of the wing imposes additional contact constraints. When coupled with the motion of the wing module, the sliding skin motion requirements will vary across the surface of the wing. Using a kinematic analysis to quantify the local effects of morphing on the wing skin is thus required for any detailed design.

\subsubsection{Stretching Skin System}

A stretchable, or flexible skin consist of a material that can stretch according to the wing morphing motion. In contrast to a sliding skin system, stretchable skins are popular primarily with twist morphing wings [23-27]. Here, the relatively small displacement of morphing bodies within the wing system are suitable for stretchable materials. A flexible material was also developed for the multi-role morphing air vehicle [44-46]. Stretchable skins have also been proposed for morphing wings with sweep and cant morphing $[13,14]$.

Implementation of a stretchable skin system has considerable challenges, particularly with respect to material selection. Kikuta [76], in his Masters dissertation, evaluated several potential materials for morphing skins. Based on experimentation, the materials tested all failed to address key issues associated with morphing, particularly with elongation, and resistance to deformation from pressure distribution. These issues, in addition to material fatigue and additional actuation 
forces, pose considerable challenges for stretchable materials for wing skins. The benefits of a stretchable skin is that the material can, in general, respond to a variety of motions.

For the wing module, a stretchable skin must be able to provide large deformations due to the span-wise motion capabilities of the module. The platform airfoil rotations each impose different motion profiles on the skin in different wing regions, thus suitable tension throughout the skin must be maintained for all wing motions to ensure the skin does not become loose (loss of tension), or become rippled. While flexible materials have been designed to address specific motions, one that allows for large extension without imposing severe actuation penalties, while simultaneously preventing rippling for shear and torsion and preventing bending from normal forces does not exist. A stretchable skin for the wing module must then be evaluated kinematically for the local motions and deformations associated with morphing, but also would require a passive support structure to prevent adverse deformation associated with morphing and pressure distribution.

\subsubsection{Hybrid Skin System}

A hybrid skin system combines elements from sliding and stretching skin systems. As such, a hybrid skin systems inherits qualities from sliding and stretching skins, making a hybrid system extremely challenging in its implementation. Theoretically, a hybrid system could be developed that is suitable for the entire morphing wing. For the wing module, for example, a sliding skin system could act as a support structure to a stretchable skin that provides an aerodynamic seal. Alternatively, the features from each skin system could be used in specific wing regions where it is most suitable, and thus easier to implement. Regardless of the design, a kinematic analysis of the skin motion must be used to lay the foundation for detailed design.

\subsubsection{Wing Skin Kinematic Response}

From a kinematic perspective, the developed kinematic formulation for the wing truss members can be extended for evaluation of the wing skin. At the highest level, Eq. (5.1) provides a vector loop between two distinct points on the base and platform airfoils. By extension, these two distinct 
point could be points on the airfoil perimeter, thus representing a span-wise skin element

$$
\mathbf{p}_{a, b}+\mathbf{a}=\mathbf{b}+\mathbf{p}_{a, p}
$$

where $\mathbf{p}_{a, b}$ and $\mathbf{p}_{p, b}$ are the base and platform airfoil perimeter vectors, and $\mathbf{a}$ is the span-wise skin vector. Here, $\mathbf{p}_{p, b}$ is expressed in the base coordinate frame where $\mathbf{p}_{p, b}=\mathbf{R} \mathbf{p}_{p, b}^{\prime}$, and $\mathbf{b}$ is still the module span vector. Equation (6.1) can be used to evaluated span-wise motion between any two points on the base and platform airfoil perimeters, though it stands to reason that the perimeter locations are equivalent for both the base and platform.

In addition to span-wise vectors, airfoil sections within the wing module centre can be defined that reflects that gradual transition from the base airfoil orientation, to the platform airfoil orientation. If $\delta$ is a fraction on the order $0 \leq \delta \leq 1$ that represents a position on the module span vector, and where $\delta=0$ and $\delta=1$ represents the base and platform airfoil positions, respectively, then the orientation of an airfoil section at $\delta$ is given by

$$
\mathbf{R}_{\delta}=\mathbf{R}_{x}(\delta \Lambda) \mathbf{R}_{y}(\delta \Gamma) \mathbf{R}_{z}(\delta \alpha)
$$

Combining span-wise skin vectors with intermediate airfoil sections results in a grid system that can be used to evaluate the kinematics and motion profile of a skin system. A discretized wing skin grid is shown in Fig. 6.2.2. Here, the grid spacing is dependent on the curvature of the airfoil cross-section with more grid elements present at the leading edge as compared to the trailing edge. As the wing module moves, the resultant motion of the skin can be observed and thus evaluated.

In the following section, the effects of individual morphing degrees-of-freedom on the wing skin are evaluated, followed by a section that evaluates the wing skin for the combined motion range of the wing module. The 4-DOF wing module with 5-6 constrained branches is used as an example for the wing skin motion analysis as this module was used for the development of the prototype. A different module mobility and constrained branch combination will naturally result in a different wing skin response, however a full analysis is beyond the scope of this dissertation. The motion 


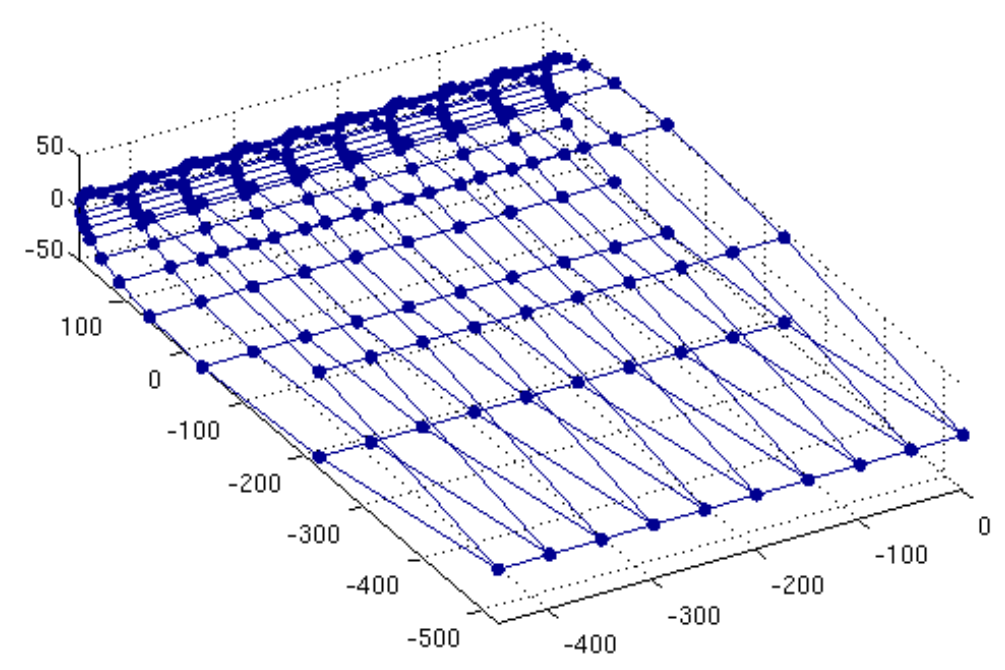

Figure 6.5: Wing module showing skin grid

analysis presented is intended to lay the foundation of a detailed design, in this particular case, for the 4-DOF wing module with 5-6 constrained branches.

The individual morphing motions each impose different requirements on the skin system. Additionally, as mentioned, the resultant motion of the wing skin is not uniform accross the entire wing surface. As such, the individual morphing motions are evaluated individually first to observe these effects. The exception to this is the span motion, where the motion response of the skin is simply identical to the span-wise motion of the wing module, thus for this analysis, the span morphing is not considered. For the analysis, the span of the module is held at $b_{z}=250 \mathrm{~mm}$. The linear expansion/retraction of span-wise grid lines and angular displacement of span-wise grid lines with respect to airfoil cross sections are analyzed. The results are normalized, based on the initial lengths and orientation of the grid lines, indicating the kinematic requirements for the skin system at different skin surface nodes. 


\subsubsection{Wing Skin Response for Individual Platform Motion}

From the kinematic analysis in Section 5.3.1.1 for the 4-DOF module with 5-6 constrained branches, the range of rotational variables present the workspace boundary for the wing module. However, these boundary values are dependent on the other platform rotations, hence the motion range for one particular platform variable with the other two held at zero is less than what is outlined in Table 5.2. In the figures that follow, the kinematic results of the skin motion are based on the range of each platform airfoil rotation, while the remaining two degrees-of-freedom are kept at a constant $0^{\circ}$. The maximum and minimum elongation of span-wise grid members, and the maximum and minimum angular displacement of intersecting grid lines are shown for each platform rotation.

The kinematic response of the wing skin for sweep motion is shown in Fig. 6.6. With the cant and twist variables held at $0^{\circ}$, the module is capable of sweeping on the interval $9^{\circ} \leq \Lambda \leq 11^{\circ}$. As expected, the maximum and minimum elongation span-wise grid values, shown in Fig. 6.6(a) and Fig. 6.6(b), respectively, are clustered near the trailing edge, peaking at just over a normalized value of 1.35 , representing a forward swept position. For an aft-swept pose, the minimum elongation values are also located at the trailing edge, with a value of approximately 0.6 of the original length. The elongation values gradually become unity towards the $1 / 4$-chord location, and then increase or decrease accordingly toward the leading edge. This is expected as the the rotation centre of the platform airfoil is at the 1/4-chord location, and indicates that any skin system implemented would not be uniform between leading and trailing edge regions with respect to extension and retraction capabilities. From an angular displacement perspective, the variation of angular displacement of grid lines varies span-wise, with maximum (Fig. 6.6(c)) and minimum (Fig. 6.6(d)) values clustered near the platform airfoil. A small region at the platform leading edge requires a very small angular displacement range, while towards the trailing edge the angular displacement of grid lines is upwards of $25^{\circ}$.

For the cant motion, the skin response is shown in Fig. 6.7. The cant motion is on the range of $-24^{\circ} \leq \Gamma \leq 27^{\circ}$ while holding the sweep and twist at a constant $0^{\circ}$. The resultant skin motion for platform cant results in maximum and minimum elongation values occuring at the wing skin regions 


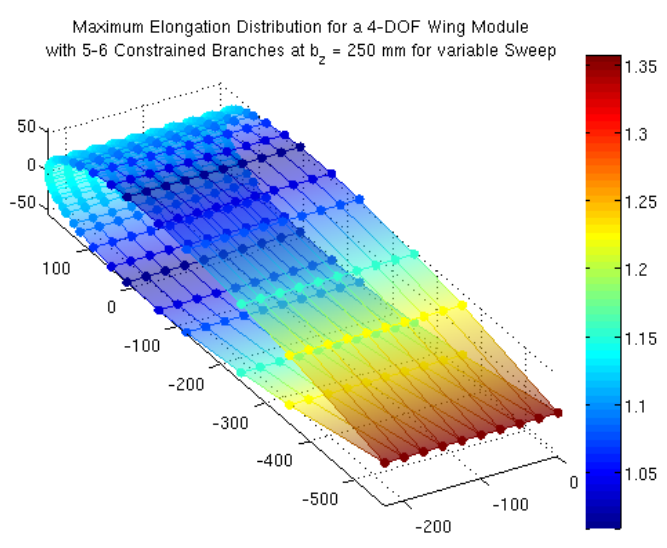

(a) Linear elongation for max sweep

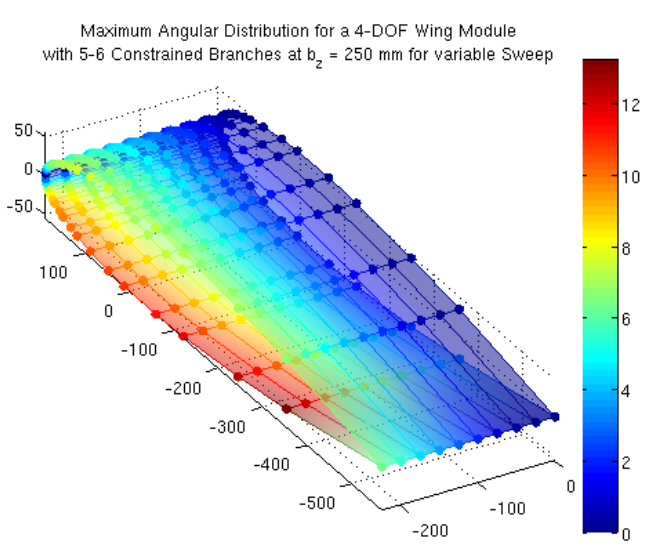

(c) Angular deformation for max sweep

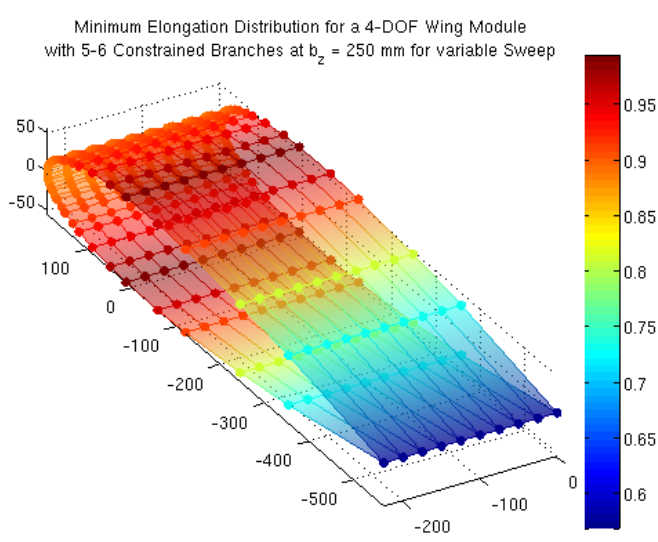

(b) Linear elongation for min sweep

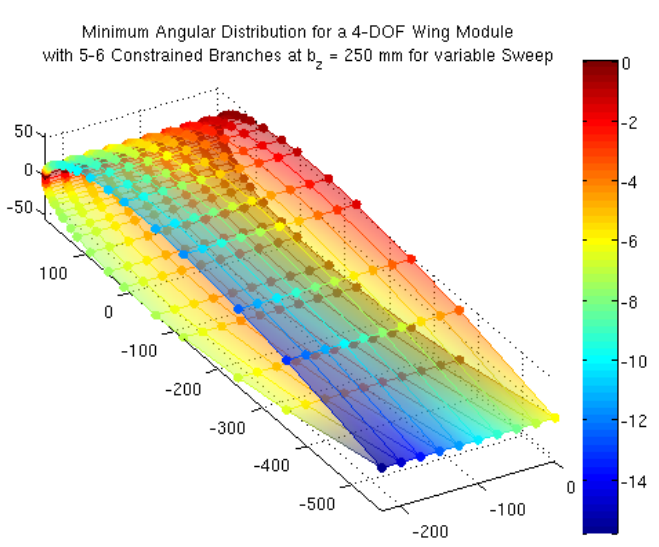

(d) Angular deformation for min sweep

Figure 6.6: Wing skin motion response for 4-DOF wing module with 5-6 constrained branches with module sweep at $b_{z}=250 \mathrm{~mm}$

furthest from the chord-line; i.e. the upper and lower wing surfaces. The maximum and minimum distribution values, shown in Fig. 6.7(a) and Fig. 6.7(b), repsectively, indicate a much smaller elongation range as compared to the distriubtion associated with sweep motion, as is expected from a geometry perspective. Here, the skin is required to expand or contract by approximately $10 \%$ of the original length while tappering off to very small values at the leading and trailing edges of the wing. The maximum and minimum angular distributions, shown in Fig. 6.7(c) and Fig. 6.7(d), respectively, show that there range of angular displacements of the wing skin is remains low for majority of the wing surface, with a very large range required at the leading edge of the platform airfoil. In this region, the combination of platform motion, and wing curvature requires 


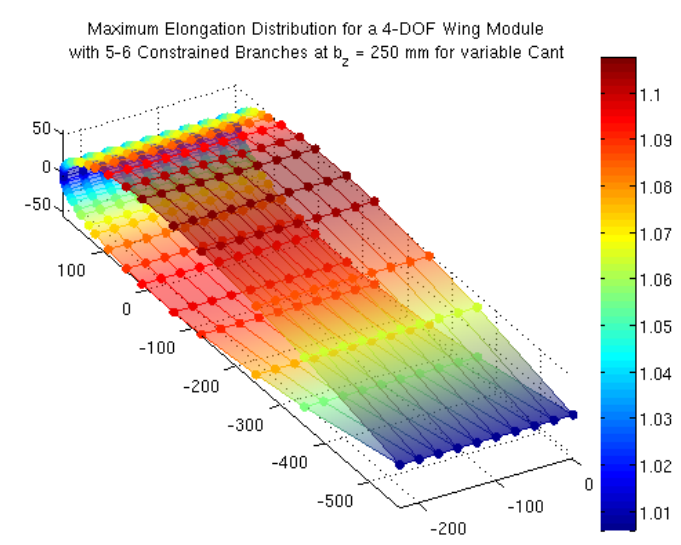

(a) Linear elongation for max cant

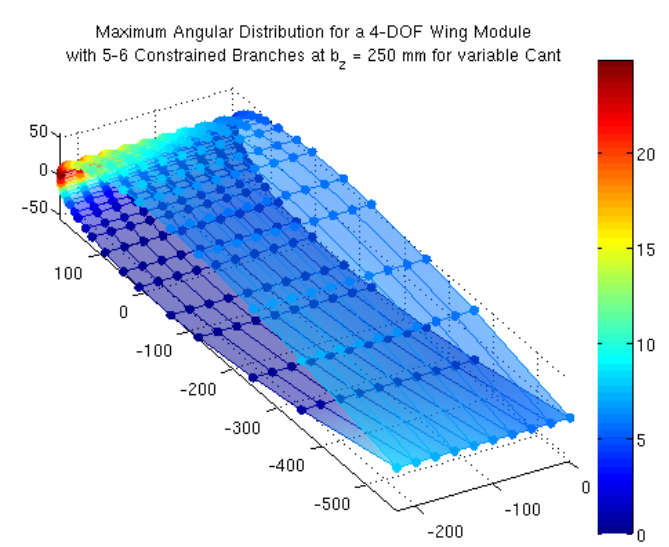

(c) Angular deformation for max cant

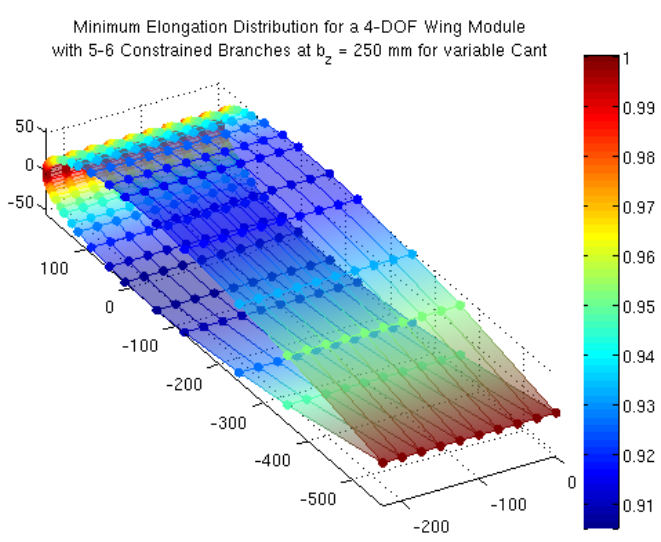

(b) Linear elongation for min cant

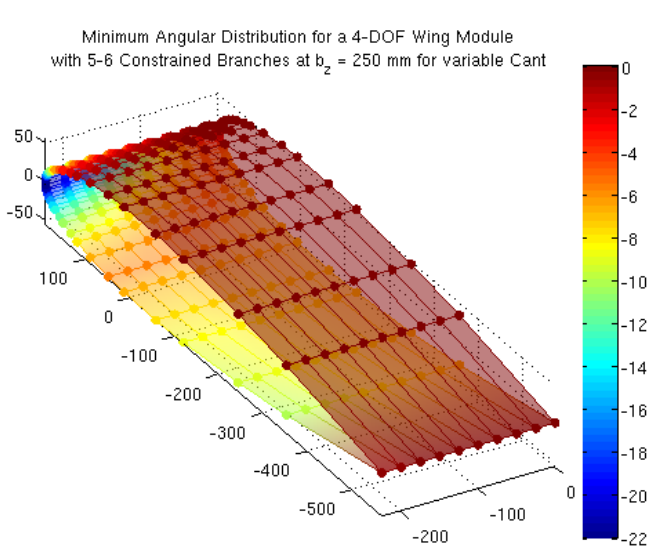

(d) Angular deformation for min cant

Figure 6.7: Wing skin motion response for 4-DOF wing module with 5-6 constrained branches with module cant at $b_{z}=250 \mathrm{~mm}$

that any developed skin must be able to withstand angular variations of upwards of $\pm 25^{\circ}$. This requirement gradually relaxes towards the base airfoil, and towards the $1 / 4$-chord. Here, the nonuniform angular displacement values between the upper and lower wing surfaces is attributed the resultant motion required by the constrained branches and the difference between maximum and minimum cant values.

Finally, the wing skin motion distribution for platform twist is shown in Fig. 6.8. Here, the platform airfoil can twist on the range $\pm 22^{\circ}$ while the sweep and cant are kept at a constant $0^{\circ}$. From the analysis, we see that for twist motion, the span-wise grid elements at no point retract, as evident by the minimum elongation distribution shown in Fig. 6.8(b). In constrast to this, there 


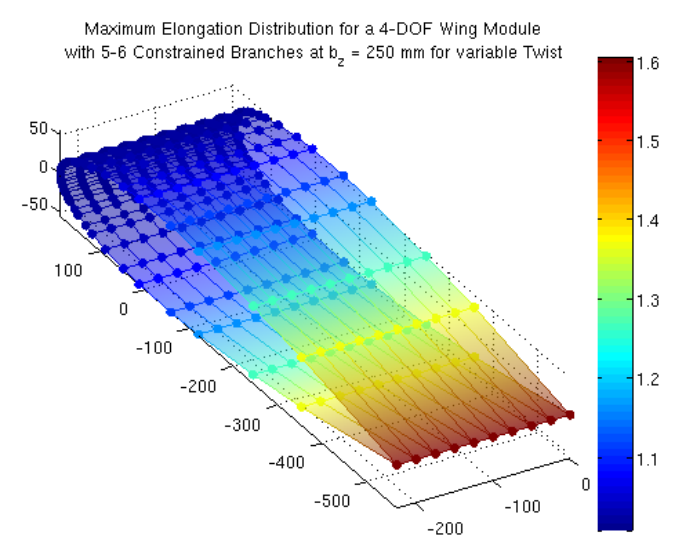

(a) Linear elongation for max twist

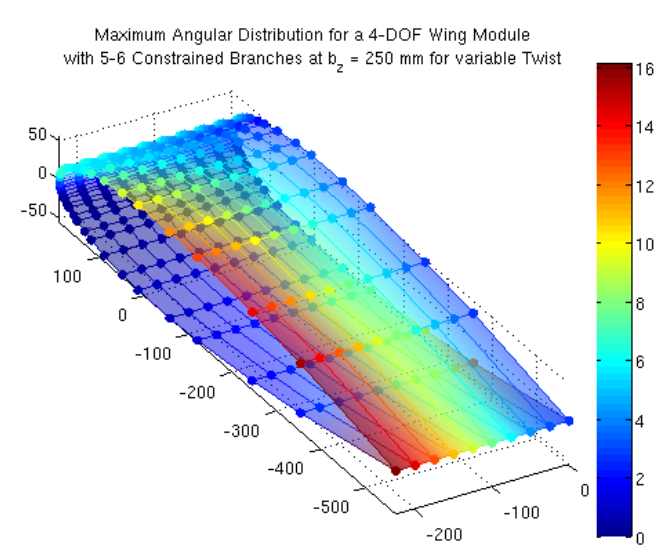

(c) Angular deformation for max twist

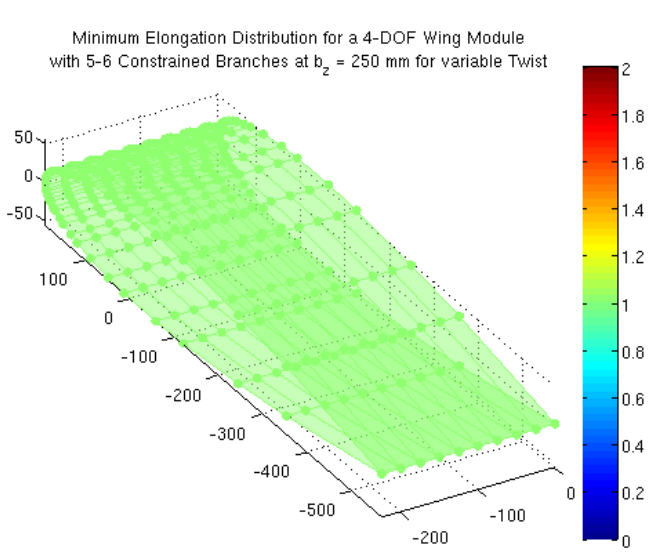

(b) Linear elongation for min twist

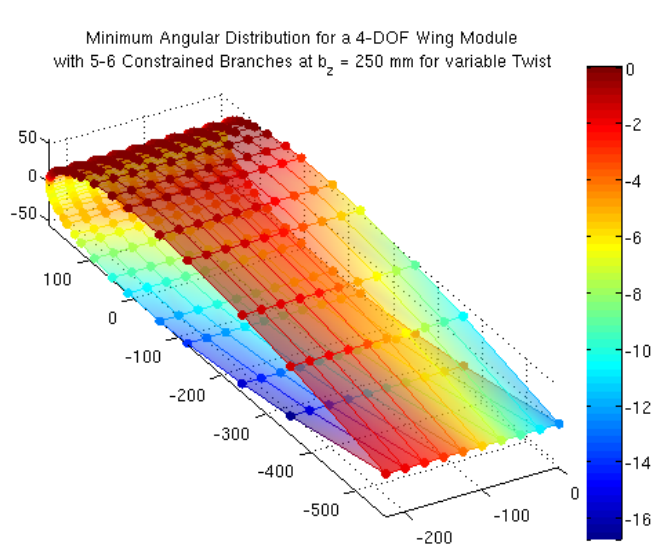

(d) Angular deformation for min twist

Figure 6.8: Wing skin motion response for 4-DOF wing module with 5-6 constrained branches with module twist at $b_{z}=250 \mathrm{~mm}$

wing skin experiences large expansions, particuarly towards the trailing edge of the module, where the wing skin is required to expand to an elongation value of approximately 1.6 (see Fig. 6.8(a)). Smaller elongation values are seen gradually towards the leading edge. The maximum and minimum angular distributions are shown in Fig. 6.8(c) and Fig. 6.8(d), respectively. Here, we see that wing twist results in a wide range of angular displacements, with maximal and minimal values located at the platform airfoil, within the middle region of the wing. The maximum and minimum values are not equal and opposite, due to the constrained kinematics, and the resultant motion of the platfom. 


\subsubsection{Wing Skin Response for Combined Platform Motion}

Building on the results in the previous section, a combination of sweep, cant and twist rotations are used for the evaluation of the wing skin motion response. Here, the kinematic range outlined in Table 5.2 of the 4-DOF wing module with 5-6 constrained branches is used to evaluate the motion response of the wing module skin and is shown in Fig. 6.9. Since the full kinematic range of the wing module at $b_{z}=250 \mathrm{~mm}$ is used, the resultant wing skin motion is not simply the superimposition of results obtained from the individual motion analysis of the previous section.

The maximum and minimum skin elongation values for the full kinematic range of the 4-DOF

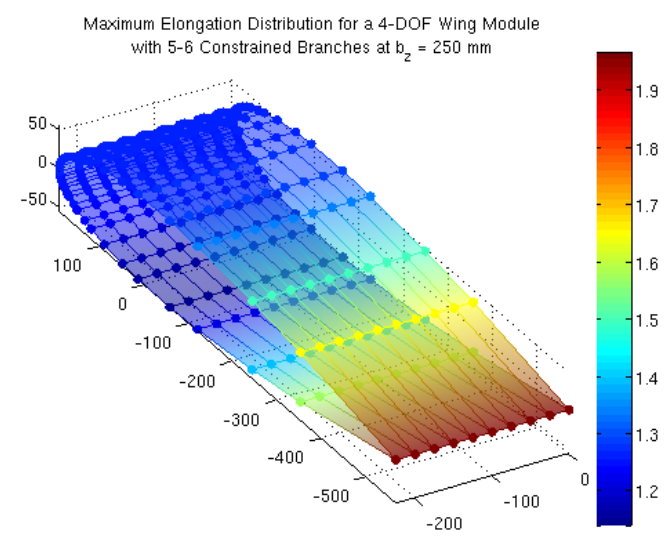

(a) Maximum linear elongation distribution

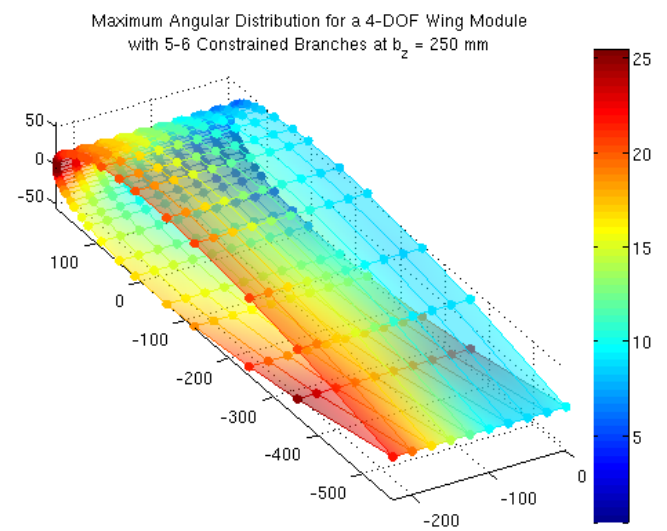

(c) Maximum angular deformation distribution

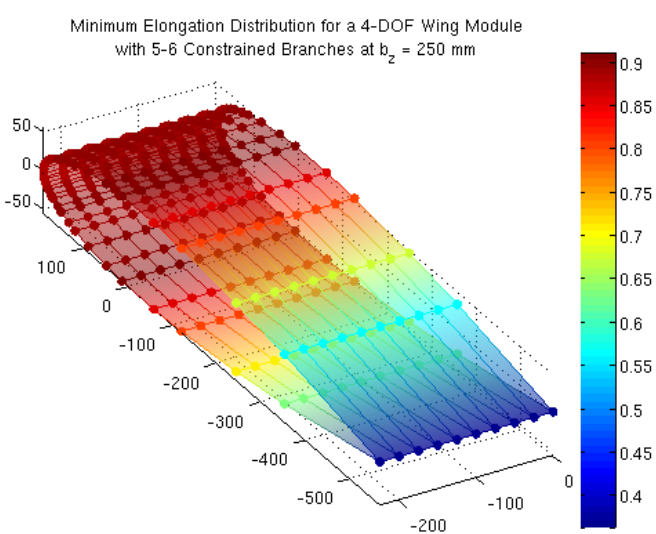

(b) Minimum linear elongation distribution

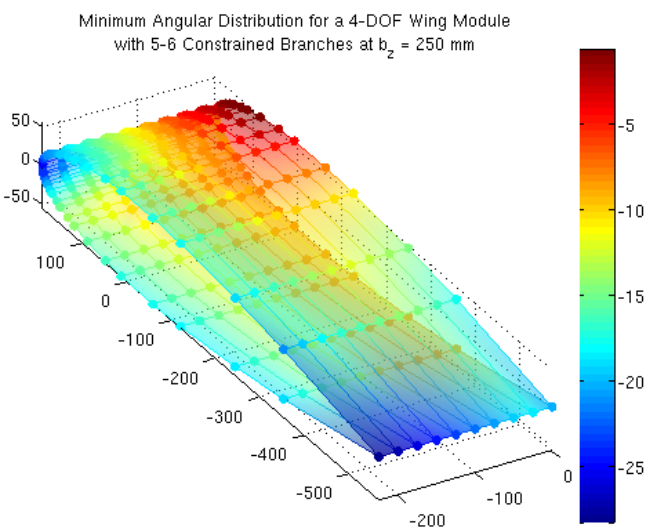

(d) Minimum linear elongation distribution

Figure 6.9: Wing skin motion response for 4-DOF wing module with 5-6 constrained branches at $b_{z}=250 \mathrm{~mm}$ 
wing module with 5-6 constrained branches are shown in Fig. 6.9(a) and Fig. 6.9(b), respectively. With a combination of platform degrees-of-freedom, we see that the resultant skin motion retains characteristics from the individual motion analyses. First, the combination of sweep and twist rotation impose greater span-wise elongation requirements on the trailing edge of the wing skin, which is expected as both individual motions required large expansions and contraction requirements in this region. With the combined motion, however, the trailing edge skin region is required to expand by almost double the original length, and retract to over half of the original length. These expansion and contraction requirements reduce towards the leading edge region of the wing. It is also shown that the regions of the wing skin near the upper and lower surfaces at the platform airfoil retain the requirements imposed by cant-morphing in which the wing skin must be able to expand or contract by $10 \%$ of the original length.

For the angular displacement, trends associated with individual morphing are also inherited when combined with other motions. The minimum and maximum angular displacements shown in Fig. 6.9(c) and Fig. 6.9(d), respectively, show that the wing skin near the platform airfoil, and particularly near the leading edge, experience the largest angular displacement for the full range of platform airfoil motions. The larger variable range results in skin angles of approximately $\pm 25^{\circ}$ near the platform airfoil leading edge, which is due not only to the curvature of the wing itself, but again, by cant morphing. Sweep and twist morphing once again imposes large skin displacement angles towards the middle of the wing.

\subsubsection{Wing Skin Motion Summary}

The resultant motion requirements of the skin system vary not only according to the motion of the platform airfoil, but vary across the wing surface itself. Large extension and retraction capabilities are required towards the leading and trailing edges, while large angular displacements tend to be present near the platform airfoil. As such, a single design paradigm might not be sufficient to address the morphing needs of the wing module. Discretizing the wing skin as presented allowed for wing skin regions to be identified in which large linear and angular displacements are required. 
Thus, the use of flexible, sliding (rigid or compliant) or hybrid skin systems can be implement in region to which they are best suited. As such, the presented analysis and kinematic formulation lays the foundation for a detailed design to be developed that meets the varied needs of the wing skin with respect to the kinematic requirements associated with morphing.

\subsection{Summary}

In this chapter, the developed morphing wing module prototype was shown. First, a discussion of the prototype was given, including a brief description of the branches of the wing module prototype. With the prototype introduced, the kinematic response of the wing module was shown in an effort to prove the validity of the kinematic formulation of Chapter 5 . Next, it was shown that the developed kinematics could be used to describe the kinematics of a wing skin system. Potential wing skin systems were introduced and their function with respect to the wing morphing degreesof-freedom were discussed. A discretized wing skin grid was then established to evaluate the motion response of the wing skin. The elongation distribution and angular displacement distribution was evaluated, first for individual wing module motions, followed by combined platform motions. It was

shown that the morphing requirements of the wing skin vary across the wing skin surface, and the kinematics can be used to lay the foundation for detailed design of a morphing wing skin system. 


\section{Chapter 7}

\section{Conclusions and Future Work}

\subsection{Conclusions}

This dissertation presented the conceptual design of a modular morphing wing. Morphing wing technology has recently become a major research focus at institutes world wide with the expressed goal of improving the performance of an aircraft during all or specific segments of its mission. Aircraft morphing can be categorized according to the degrees-of-freedom which morphing provides. At the wing level, these morphing degrees-of-freedom include variable sweep, cant, twist and span. Within the literature, numerous concepts have been proposed, each addressing the morphing problem in a different manner, but primarily focusing on a single, or specific set of morphing degrees-of-freedom. The proposed mechanisms and methods of morphing are so specific that new systems must be developed in order to address a different individual or set of morphing degreesof-freedom. Additionally, for many projects, a morphing mechanism was developed first, followed by a performance analysis of the wing morphing mechanism. This process thus limits the potential for maximizing performance gains that the morphing wing was originally required to provide. The developed modular morphing wing concept presented in this dissertation aimed to address these specific issues.

The proposed morphing wing module is based structurally and kinematically on variable geom-

etry truss mechanisms. In general, a variable geometry truss is a sequential stacking of closed-loop parallel mechanism modules to form an open-loop serial-style mechanism. Truss mechanisms in- 
herit positive qualities from each parent mechanism, which was shown to be beneficial when applied in a morphing wing scenario. From serial robots, a truss mechanism inherits large dexterity and workspace reach, which for a morphing wing, is essential for providing large scale morphing maneuvers. Each module of the truss mechanism is an individual parallel mechanism which provides high stiffness, and payload-to-weight ratios to the truss system, which is more capable of withstanding the loads associated with flight, as compared to a simple serial mechanism. The truss platform provided the needed qualities listed above, such that the morphing wing system could be developed in a modular fashion.

Further to this, since the morphing wing system consists of sequentially stacked modules, the analysis of the system reduced to a single module only. The module itself was designed as a reconfigurable parallel truss system, capable of not only altering the number of degrees-of-freedom for the wing module, but also the combination of these degrees-of-freedom. As such, the developed system has a very large degree of flexibility in configuration generation as modules ranging from two, up to a combination of up to four degrees-of-freedom are possible. Span-morphing was shown to be a requirement for all modules. Using the four wing-level morphing degrees-of-freedom, the base wing module is capable of reconfiguring itself to provide any above degree-of-freedom combination. This allows for a morphing wing system to be quickly generated to match any degree-of-freedom combination required, at any station along the wing span, without the need to develop a new wing morphing system from scratch. This not only saves time and development costs, but allows the wing system to address the specific morphing needs for a specific mission, and thus increase the potential performance gains as compared to other morphing wings.

\subsection{Contributions}

The main contributions of this dissertation are as follows:

\section{Modularity and Reconfigurability within the Morphing Wing Field}

First and foremost, the subsequent contributions are due to the introduction of modularity 
and reconfigurability within the morphing wing field. These concepts are prevalent within the robotics field where much research has been conducted. However for the morphing wing field, designs in the literature were proposed for specific morphing characteristics. The introduction of modularity and reconfigurability addressed a specific need where a morphing wing could be quickly designed to address specific morphing requirements. This laid the foundation for further research and development of a modular wing system in which analysis tools could be developed at the module level, and be easily applied to all permutations of the wing module configuration.

\section{Morphing Wing Truss}

Building on the concepts of modularity and reconfigurability, a reconfigurable modular truss mechanism was proposed to provide a wing with specific morphing degrees-of-freedom at specific span-wise locations. The individual truss modules were designed to be reconfigurable and to provide any combination of sweep, cant, and twist with span morphing capabilities.

Sequentially stacking specific truss modules could then be used to form a unique morphing wing system. This modularity and reconfigurability allows for an unlimited number of morphing wings to be quickly designed and analyzed, thus eliminating the need to design specific mechanisms for specific morphing degrees-of-freedom.

\section{Discretization Algorithm}

A discretization algorithm was presented that discretizes an otherwise continuous wing, into a discrete number of wing modules. The presented algorithm determines the minimum number of wing modules required to emulate a known and desirable wing shape and associated performance. Additionally, the algorithm used the known geometry of a reference wing to provide a natural and intelligent spacing of wing modules in which fewer modules were clustered in wing regions with low curvature and/or twist, and additional modules were clustered in wing regions with high curvature and/or twist. A mathematical description of the reference wing was provided using the parallel transport method of curve framing. The algorithm incrementally increased the number of modules within the wing system and the aerodynamic 
performance was analyzed until a maximum performance was achieved, thus yielding the minimum number of wing modules for the morphing wing system.

\section{Branch Based Mobility Analysis}

The modules of the proposed wing system are based off parallel robot architecture, in which branch modules are assembled in parallel to form the mechanism. Mobility was traditionally analyzed based on the number of joints and links within the robot system, however the inherit modularity of branches with the parallel robot architecture was exploited, and the mobility equation was redeveloped in terms of the number of unconstrained and constrained branches within the wing system. For the two proposed parallel robot constraint methodologies it was shown that the mobility of the system reduced to either the connectivity of the constraint branch within tricept-style parallel robots, or to the number of constrained branches within the robot structure for Stewart-style parallel robots. It was the Stewart-style, or joint constraint methodology, that was used for the basis of the wing module.

\section{Reconfigurable Parallel Mechanism}

The presented mobility analysis allowed for new joint-constraint parallel mechanisms to be developed conceptually in which the number of branches within the system were not equal to the mobility of the system. In fact, it was shown that an infinite number of unconstrained branches could be placed within the parallel mechanisms without constraining the motion of the platform. By using hybrid prismatic joints, and reconfigurable universal-to-revolute joints, reconfiguration to lower mobility modes is achievable for the parallel mechanism without the need to remove branches from the system. The wing module was designed with this feature, and a specialized rectangular parallel-style truss was proposed. The module consisted of a set unconstrained-active, unconstrained-passive and constrained-passive branches which could be reconfigured not only to lower mobility modes, but could do so independently of the selection of dependent platform variables.

\section{Parametric Constraint Kinematics}


A parametric constraint kinematic formulation was presented that was applied to the wing module. The parametric constraint formulation can also be applied to a wide variety of lower mobility parallel robots. Applied to branches that are constrained to move within a plane, the parametric constraint equation can be used for any constrained branch orientation and is independent of the selected independent and dependent platform variables. Additionally, the parametric constraint was further formulated to describe the instantaneous kinematics of the lower mobility parallel mechanisms. Formulation of the constrained Jacobian matrix was also shown.

\section{Prototype Development}

Finally, through the development of a mechanical prototype wing module, the branch based mobility, reconfigurable parallel mechanism and parametric constraint kinematics were proved to be valid. It was also shown that the developed kinematics could be used to describe the motion response of a wing skin system. Through a search of workspace variables, it was shown that the kinematic response of the wing skin system is not uniform across the wing surface with respect to skin elongation and angular displacement. The analysis shows that the kinematic analysis can be used a foundation for the detailed design of a morphing wing skin system.

\subsection{Future Work}

The work presented in this dissertation provides a base for further analysis of the morphing wing module. Here, high level design concepts were presented and there is opportunity to build on these concepts. Some relevant future works are described below.

First, the presented discretization method determines the number of required modules for a single wing profile. For each segment of an aircraft mission, the discretization algorithm would yield a different number of required modules. Additionally, the spacing of the modules would be different for each mission segment. A method is therefore required to consolidate the different module configurations into a single set of morphing wing modules. In doing so, the unique wing module 
configuration would provide near-optimal performance for all of the mission segments. Finally, an iterative approach could be implemented to fine tuning the final wing module configuration to reduce the controllable degrees-of-freedom within the wing system, i.e. introducing the constraint kinematic, and lower mobility module configurations to the discretization algorithm. The ability to reduce the total degrees-of-freedom for all wing modules while minimizing effects on the overall performance of the wing is ideal from a complexity standpoint, and also better utilizes the reconfigurability of the wing module.

Next, it was mentioned in Chapter 5 that the orientation of the constraint planes could be adjusted to provide a better kinematic response. It was shown that the kinematic performance of the wing module is dependent on the combination of constrained branches, and the respective constraint plane orientations. An optimization algorithm could therefore be used to determine optimal constraint plane orientations, thus improving the kinematic performance.

A kineto-structural analysis of the wing truss system is required, particularly for truss member sizing. Naturally, modules at the wing base must be stronger to withstand the aerodynamic forces experience during flight. Additionally, the dynamics, vibration and flutter analysis is essential in ensuring the structural stability of the wing during flight. These analyses could be combined with a constraint plane orientation analysis in a MDO analysis to fully consider all aspects of the truss structure for a final configuration for each wing module configuration.

Further efforts into developing a viable skin system is required to achieve a flight-ready design. Initial efforts in developing a flexible skin system proved to impose a large actuation penalty on the truss mechanism. While an brief kinematic analysis of the skin motion response was presented, further analysis into the kinematics, kineto-statics and dynamics of a suitable system is required.

Finally, from an aerodynamic perspective, there are considerable aerodynamic issues to be explored, but were beyond the scope of this dissertation. First, the discrete nature of the wing modules present areas on the wing which are discontinuous, and the associated effects and possibly penalties could be evaluated. Additionally, an analysis when transitioning between wing shapes must be achieved to ensure the transition does not adversely effect the aerodynamics and stability 
of the aircraft. These analyses can be done through computational tools and confirmed through wind tunnel testing. Though not mentioned previously, a static wind tunnel model was evaluated. With progression in developing a viable skin system, a mechanical wind tunnel model could be developed. 
Appendices 


\section{Appendix A}

\section{CFD Mesh}

In Chapter 3, a hypothetical reference wing was used to evaluate the aerodynamic performance of a morphing wing. Computational fluid dynamics was used to compute the aerodynamic forces for each wing configuration. Meshing was computed using ANSYS@ Gambit ${ }^{\mathrm{TM}}$ on the HPCVL network.

Due to the memory limitations of the 32 bit version of the Gambit software, the computation grid had to be split into smaller volumes to allow the mesh file to be saved without running out memory. The computational grid used for the aerodynamic analysis is divided into three main regions. With each iteration of the discretization loop, the exact size of the computation grid varied according to the wing geometry. Approximately 12 million cells were used for each iteration which is depictred in Fig. A.1. The details of each region is described below.

Interior Structured Wing Boundary Region The region directly surrounding the wing was a structured hexahedral mesh as shown in Fig. A.1(a) and Fig. A.1(b). This region forms an ellipse that bounds the volume of the wing and is scaled according to the wing taper, which also scales the mesh accordingly. For each airfoil rib plane, 120 mesh nodes are distributed along the upper and lower surfaces of the wing, with nodes clustered at the leading and trailing edge sections. A total of 50 grid layers extend out from the wing surface perpendicularly The spacing of the off wall layers were decreased until a $y^{+} \approx 1$ was acheived. In the span-wise direction, the nodal spacing for each modules was adjusted to acheive a uniform quadrilateral 

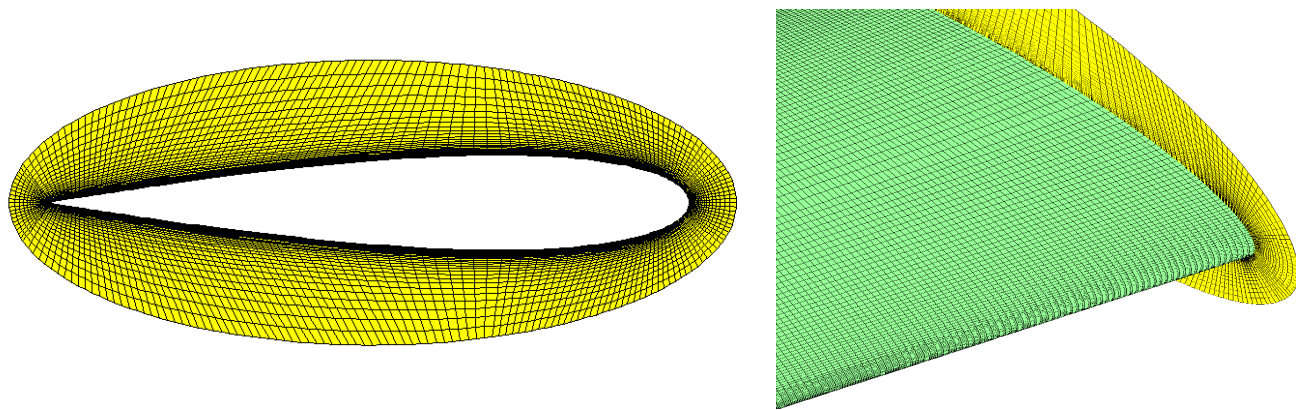

(a) Interior structured wing boundary mesh airfoil rib (b) Wing surface interior structured wing boundary cross section

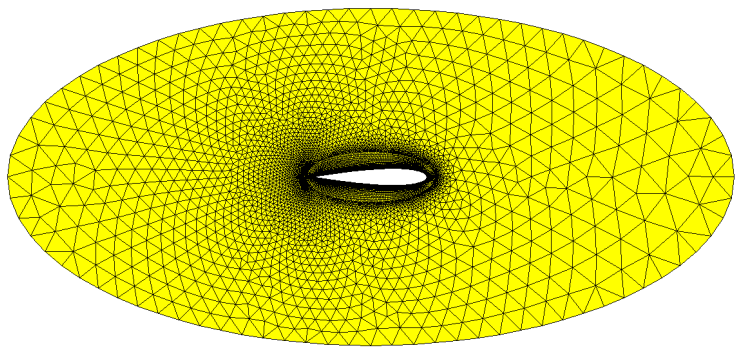

(c) Middle unstructured symmetry mesh

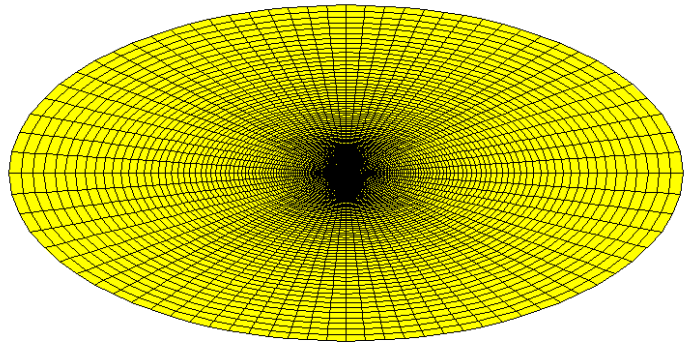

(e) Far-field structured symmetry mesh mesh

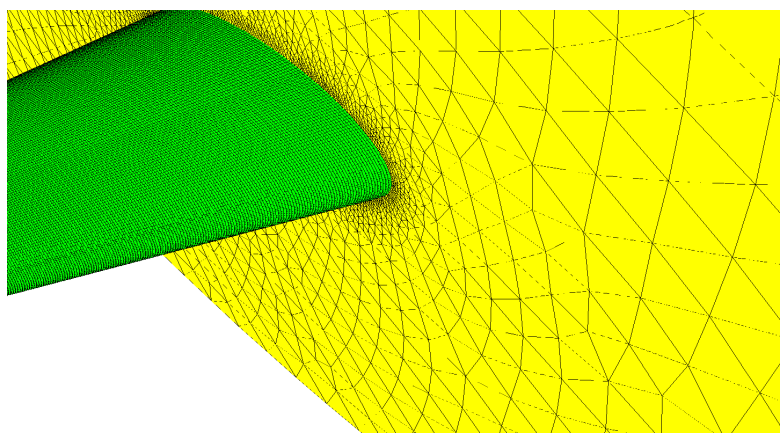

(d) Boundary between interior structured and middle unstructured meshes

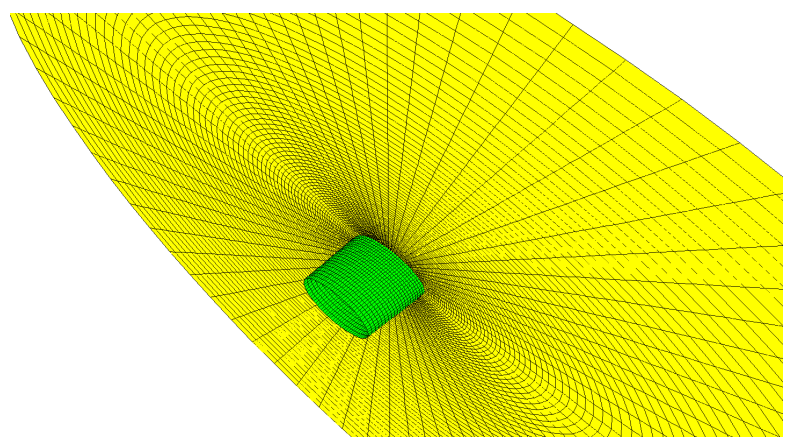

(f) Boundar between middle unstructured and far-field structured grid

Figure A.1: Computational grid used for the discretization algorithm performance analysis

surface mesh on the upper and lower surface of the wing.

Middle Unstructured Region Growing off of the interior structure wing boundary region was the middle unstructured tetrahedral region as shown in Fig. A.1(c) and Fig. A.1(d). The flexibility of the unstructred tetrahedral elements permitted a smooth growth of mesh elements outward from the wing. In addition to this, the unstructured grid was capable of providing a suitable mesh around the interior structured wing boundary region for each wing config- 
uration analyzed. The middle unstructured region was an ellipse with a three chord length major axis, and one and half chord length minor axis. The major axis was colinear with the wing root chord. Span-wise, the computation grid extended five chord lengths.

Far-field Structured Region The final region of the mesh was the far-field hexahedral structured region which extended out from the boundary of the middle unstructured region. The far-field structured rgion was also elliptical in shape with a 50 chord length major axis, and 25 chord length minor axis. As with the middle unstructured region, the major axis was colinear with the wing root chord, and the extened five chord lengths spanwise. The far-field unstructured grid is shown in Fig. A.1(e) and Fig. A.1(f). 


\section{Appendix B}

\section{Parallel Robot Branch Enumeration}

In Chapter 4, the kinematic architecture of the wing modules are given. As the wing module is based off of reconfigurable parallel robots, the module itself is defined by the branch architecture. The focus of the architecture analysis was on module configuration. Here, details relating to branch architecture is given as it pertains to the reconfiguration of parallel mechanisms.

\section{B.1 Parallel Mechanism Branch Candidate Enumeration}

When designing a parallel robot, Eq. (4.2) must be considered in addition to Eq. (4.1). As the hexapod has a system order $M=6$, and the system order $\lambda=6$, the resultant connectivity for each of the six branches within the system is $C_{k}=6$. While any combination of links and joints can be used to for the branches, not all combinations are suitable. The resultant screw system for the branch must allow the platform to move and orientate itself independently within three dimensional space. Table B.1 depicts various kinematic pairs (joints) that can be used to fulfill the connectivity requirements.

Using the kinematic pairs listed in Table B.1, possible branches can be enumerated. Branch candidates were enumerated using the joints listed in Table B.1 for an on-the-fly reconfigurable robot [57]. In general though, the various joints can be assembled in any sequence such that the summation of their individual degrees-of-freedom satisfy the connectivity requirements outlined in Chapter 4. Given that the lowest connectivity of any kinematic pair is unity, and the highest 
Table B.1: Summary of common kinematic pairs

\begin{tabular}{|c|c|c|c|}
\hline Kinematic Pair & Abbreviation & Kinematic Diagram & Degrees-of-Freedom \\
\hline $\begin{array}{l}\text { Transverse Axis } \\
\text { Revolute }\end{array}$ & $\mathrm{R}_{\mathrm{T}}$ & & 1 \\
\hline Axial Axis Revolute & $\mathrm{R}_{\mathrm{A}}$ & & 1 \\
\hline $\begin{array}{l}\text { Variable Leg Length } \\
\text { Prismatic }\end{array}$ & $\mathrm{P}_{\mathrm{V}}$ & & 1 \\
\hline $\begin{array}{l}\text { Fixed Leg Length } \\
\text { Prismatic }\end{array}$ & $\mathrm{P}_{\mathrm{F}}$ & & 1 \\
\hline Universal (Hooke) & $\mathrm{U}$ & & 2 \\
\hline Cylindrical & $\mathrm{C}$ & & 2 \\
\hline Spherical & S & & 3 \\
\hline
\end{tabular}

connectivity is three, the minimum and maximum number of joints within a given branch is two and six, respectively for a branch connectivity $C_{k}=6$. The number of joints within the branch system is inversely proportional to the robot stiffness and proportional to the robot workspace and dexterity. It is for this reason that most parallel robots adhere to a two-link, three-joint branch architecture $[56,57,71]$, although others have been proposed $[49,50,55,61,70]$. A enumerated list of possible branch configurations is given in Table B.2. For simplicity, the branches are listed using their associated joint abbreviations, in order from the base to the moving platform. For instance, a $\mathrm{P}_{\mathrm{F}} \mathrm{US}$ branch consists of a fixed prismatic joint attached at the base, a universal joint within the middle of the branch, followed by a spherical joint attached to the moving platform. 
Table B.2: Enumeration of all possible parallel robot branch candidates with two-link, three-joint branch architecture

\begin{tabular}{|c|c|c|c|}
\hline \multicolumn{4}{|c|}{ Degree-of-Freedom } \\
\hline Joint 1 & Joint 2 & Joint 3 & Branch Candidates \\
\hline 1 & 2 & 3 & $\mathrm{R}_{\mathrm{T}} \mathrm{US}, \mathrm{R}_{\mathrm{T}} \mathrm{CS}, \mathrm{R}_{\mathrm{A}} \mathrm{US}, \mathrm{R}_{\mathrm{A}} \mathrm{CS}, \mathrm{P}_{\mathrm{V}} \mathrm{US}, \mathrm{P}_{\mathrm{V}} \mathrm{CS}, \mathrm{P}_{\mathrm{F}} \mathrm{US}, \mathrm{P}_{\mathrm{F}} \mathrm{CS}$ \\
\hline 1 & 3 & 2 & $\mathrm{R}_{\mathrm{T}} \mathrm{SU}, \mathrm{R}_{\mathrm{T}} \mathrm{SC}, \mathrm{R}_{\mathrm{A}} \mathrm{SU}, \mathrm{R}_{\mathrm{A}} \mathrm{SC}, \mathrm{P}_{\mathrm{V}} \mathrm{SU}, \mathrm{P}_{\mathrm{V}} \mathrm{SC}, \mathrm{P}_{\mathrm{F}} \mathrm{SU}, \mathrm{P}_{\mathrm{F}} \mathrm{SC}$ \\
\hline 2 & 1 & 3 & $\mathrm{UR}_{\mathrm{T}} \mathrm{S}, \mathrm{UR}_{\mathrm{A}} \mathrm{S}, \mathrm{UP}_{\mathrm{V}} \mathrm{S}, \mathrm{UP}_{\mathrm{F}} \mathrm{S}, \mathrm{CR}_{\mathrm{T}} \mathrm{S}, \mathrm{CR}_{\mathrm{A}} \mathrm{S}, \mathrm{CP}_{\mathrm{V}} \mathrm{S}, \mathrm{CP}_{\mathrm{F}} \mathrm{S}$ \\
\hline 2 & 3 & 1 & $\mathrm{USR}_{\mathrm{T}}, \mathrm{USR}_{\mathrm{A}}, \mathrm{USP}_{\mathrm{V}}, \mathrm{USP}_{\mathrm{F}}, \mathrm{CSR}_{\mathrm{T}}, \mathrm{CSR}_{\mathrm{A}}, \mathrm{CSP}_{\mathrm{V}}, \mathrm{CSP}_{\mathrm{F}}$ \\
\hline 2 & 2 & 2 & UUU, UUC, UCU, UCC, CCC, CCU, CUC, CUU \\
\hline 3 & 1 & 2 & $\mathrm{SR}_{\mathrm{T}} \mathrm{U}, \mathrm{SR}_{\mathrm{T}} \mathrm{C}, \mathrm{SR}_{\mathrm{A}} \mathrm{U}, \mathrm{SR}_{\mathrm{A}} \mathrm{C}, \mathrm{SP}_{\mathrm{V}} \mathrm{U}, \mathrm{SP}_{\mathrm{V}} \mathrm{C}, \mathrm{SP}_{\mathrm{V}} \mathrm{U}, \mathrm{SP}_{\mathrm{F}} \mathrm{C}$ \\
\hline 3 & 2 & 1 & $\mathrm{SUR}_{\mathrm{T}}, \mathrm{SUR}_{\mathrm{A}}, \mathrm{SUP}_{\mathrm{V}}, \mathrm{SUP}_{\mathrm{F}}, \mathrm{SCR}_{\mathrm{T}}, \mathrm{SCR}_{\mathrm{A}}, \mathrm{SCP}_{\mathrm{V}}, \mathrm{SCP}_{\mathrm{F}}$ \\
\hline
\end{tabular}

In total, 56 different branch configurations are possible that satisfy the connectivity requirements of the system, however not all candidate configurations succeed at satisfying the physical motion requirements of its associative screw system. As mentioned, the branch must allow the platform to move and orientate itself freely within three-dimensional space. To satisfy this requirement, enumeration rules are applied to the possible branch configurations in order to eliminate unfeasible configurations. In addition to these enumeration rules, others are applied that address implementation issues associated with physical characteristics of the joints, as well as rules that grant positive kinematic qualities to the robot system.

\section{Spherical Joints}

From a kinematic and kineto-static perspective, it is advantageous to have a spherical joint occupy the Joint 3 position within the branch. Kinematically, a spherical joint attached to the platform guarantees the platform is capable to freely orientate itself with respect to the branch. The two remaining joints need only independently position the spherical joint within three-dimensional space in order to fulfill the kinematic requirements of a branch. From a kineto-static perspective, the spherical joint does not transmit moments to the branch; only linear forces are transmitted. As such, a spherical joint is required to be positioned at the 
Joint 3 location. Any branches that do no satisfy this enumeration rule are eliminated.

\section{Variable and Fixed Prismatic Joints}

The nature of variable leg length and fixed leg length prismatic joints require that the fixed leg length prismatic style actuator must be physically connected to some other component, for example, the base. If a prismatic joint is to be placed as the second joint within the branch, then a variable leg length prismatic joint is required, as these prismatic actuators do not require a physical connection point. Conversely, a variable leg length prismatic joint connected to the base is in effect, a fixed leg length prismatic actuator. Using this, the enumeration rule that Joint 1 prismatic actuators are of the fixed leg length type, and Joint 2 prismatic actuators are of the variable leg length type. Any branches that do not follow this rule are eliminated.

\section{Axial Revolute Joints}

With a spherical joint required at the platform level, an axial revolute joint within the branch does not guarantee that the platform can position and orientate itself freely with respect to the branch. This is a result of the rotation axis of the axial axis revolute joint and link being colinear. An axial axis revolute joint preceded or followed by a universal joint does not allow the moving platform the necessary motion as described above. This is also true when an axial axis revolute joint follows a cylindrical joint. As such, an axial axis revolute joint is only acceptable when it is followed by a cylindrical joint whose linear axis is not parallel to the rotation axis of the axial axis revolute joint. This final enumeration rule eliminates any branches with adjacent axial axis revolute and universal joints and any branch with a cylindrical joint followed by an axial axis revolute joint.

After the enumeration rules are applied and unsuitable branches eliminated, nine branch candidates remain which are listed in Table B.3. These branches satisfy the connectivity requirements imposed by Eq. (4.2), while satisfying the kinematic requirement for the moving platform. As such, any of the branches listed can be used to form a full 6-DOF hexapod. 
Table B.3: Enumeration of feasible parallel robot branch candidates with two-link, three-joint branch architecture

\section{Degree-of-Freedom}

\section{Joint 1 Joint 2 Joint 3 Branch Candidates}

\begin{tabular}{llll}
\hline 1 & 2 & 3 & $\mathrm{R}_{\mathrm{T}} \mathrm{US}, \mathrm{R}_{\mathrm{T}} \mathrm{CS}, \mathrm{R}_{\mathrm{A}} \mathrm{CS}, \mathrm{P}_{\mathrm{F}} \mathrm{US}, \mathrm{P}_{\mathrm{F}} \mathrm{CS}$ \\
2 & 1 & 3 & $\mathrm{UR}_{\mathrm{T}} \mathrm{S}, \mathrm{UP}_{\mathrm{V}} \mathrm{S}, \mathrm{CR}_{\mathrm{T}} \mathrm{S}, \mathrm{CP}_{\mathrm{V}} \mathrm{S}$ \\
\hline
\end{tabular}

\section{B.2 Parallel Mechanism Constrained Branch Candidate Enumer- ation}

While the branches listed in Table B.3 can be used to form a 6-DOF parallel robot, modifications must be made to form parallel robots of lower mobility as constraints must be added to the system. Constraints are added to the system by removing a degree-of-freedom from the system. The unconstrained branch candidates listed in Table B.3 each consist of three joints. As seen, the branch candidates possess a joint degree-of-freedom sequence of either $f_{j}=1,2,3$, or $f_{j}=2,1,3$, and each joint is a candidate for a degree-of-freedom to be removed. Removing a degree-of-freedom introduces the necessary system constraint that allows a robot to assume a lower mobility mode, however the physical ramifications differ depending on which joint the degree-of-freedom is removed from. From a connectivity perspective, one of three options are available as discussed below.

\section{1-DOF joint reconfigures to a 0-DOF joint}

Removing the degree-of-freedom from the joint of $f_{j}=1$ (either $\mathrm{R}_{\mathrm{T}}, \mathrm{R}_{\mathrm{A}}, \mathrm{P}_{\mathrm{F}}$, or $\mathrm{P}_{\mathrm{V}}$ ) eliminates the joint from the branch, and results in the two links to be fused into a single link. This reconfiguration reduces the branch to a one-link, two-joint system. The resulting branch configurations that that satisfy the $f_{j}=2,3$ joint degree-of-freedom sequence are either of US or CS architecture. For the US branch, the spherical joint is restricted to move on the surface of a sphere whose radius is defined by the length of the link connecting the universal and spherical joints. The CS branch on the other hand requires the spherical joint moves along the curved surface of a cylinder whose radius is defined by the link connecting the cylindrical and spherical joints. Though kinematically feasible, it becomes increasingly difficult to achieve a 
suitable kinematic response with each additional constrained branch, of either configuration. For instance, branches of US architecture impose a finite limit on the height of the platform with respect to the base due to the fixed link lengths. Branches of CS architecture on the other hand could allow height adjustment of the platform, but lateral motion is increasingly more difficult with reduction in system mobility.

\section{2-DOF joint reconfigures to a 1-DOF joint}

A degree-of-freedom can be removed from the 2-DOF joint, resulting in a branch with a joint degree-of-freedom sequence of $f_{j}=1,1,3$. The two, 2-DOF joints are the universal and cylindrical joints. For the universal joint, removing a degree-of-freedom results in a transverse axis revolute joint, regardless of which rotation axis is removed. The cylindrical joint on the other hand has two distinct reconfigurations as the joint itself consists of one linear and one rotation axis. Thus after reconfiguration, the resultant joint is either a transverse axis revolute joint, or a fixed leg length or variable leg length prismatic joint (depending on where the cylindrical joint was location within the branch). Branches with a joint degree-of-freedom sequence of $f_{j}=1,1,3$ are very common in lower mobility parallel robots. For example, $[48,54,56,57,77]$ all present parallel robots that have joint degree-of-freedom sequence $f_{j}=$ 1, 1,3 for lower mobility parallel robots.

\section{3-DOF joint reconfigures to a 2-DOF joint}

The final option available is removing a degree-of-freedom from the 3-DOF joint. The only option available here is the spherical joint, and reconfiguration results in the spherical joint at the platform becoming a universal joint with branches assuming either a $f_{j}=2,1,2$, or $f_{j}=1,2,2$ joint degree-of-freedom sequence. Configurations such as this have been proposed in some parallel robots, for instance [61]. This reconfiguration maintains the two-link, threejoint architecture of the parent branch, but the loss of a rotation degree-of-freedom at the platform will allow bending about one axis to be passed to the upper link within the branch. 
From the above analysis, we can see that removing a degree-of-freedom from the 1-DOF joint within the branch is not a viable option as the resultant motion of the platform becomes increasingly restricted as the robot assumes a lower mobility mode. Thus this option is discarded from further analysis. Removing a degree-of-freedom from the spherical joint to have a universal joint at the platform, although feasible, is discarded here due to structural considerations of passing bending from the platform to the branches. Removing a degree-of-freedom from the 2-DOF joint within the branch is thus the preferred method to introduce constraints within the robot system.

In order to apply the constraints, a degree-of-freedom is removed from either a universal or cylindrical joint. Equation (4.8) is independent of the branch configuration, but it is important to expand on the reconfiguration of the branch when a degree-of-freedom is removed, a summary of which is shown in Table B.4. The final configurations are sorted based on how the universal or cylindrical joint is reconfigured, and after reconfiguration there are six unique configurations available: $\mathrm{R}_{\mathrm{T}} \mathrm{R}_{\mathrm{T}} \mathrm{S}, \mathrm{R}_{\mathrm{T}} \mathrm{P}_{\mathrm{V}} \mathrm{S}, \mathrm{R}_{\mathrm{A}} \mathrm{R}_{\mathrm{T}} \mathrm{S}, \mathrm{R}_{\mathrm{A}} \mathrm{P}_{\mathrm{V}} \mathrm{S}, \mathrm{P}_{\mathrm{F}} \mathrm{R}_{\mathrm{T}} \mathrm{S}$, and $\mathrm{P}_{\mathrm{F}} \mathrm{P}_{\mathrm{V}} \mathrm{S}$.

Table B.4: Reconfiguration of branch candidates for reduced mobility modes

\begin{tabular}{ll}
\hline Joint & Reconfigured Branch Configuration \\
\hline $\mathrm{U} \rightarrow \mathrm{R}_{\mathrm{T}}$ & $\mathrm{R}_{\mathrm{T}} \mathrm{US} \rightarrow \mathrm{R}_{\mathrm{T}} \mathrm{R}_{\mathrm{T}} \mathrm{S}, \mathrm{P}_{\mathrm{F}} \mathrm{US} \rightarrow \mathrm{P}_{\mathrm{F}} \mathrm{R}_{\mathrm{T}} \mathrm{S}, \mathrm{UR}_{\mathrm{T}} \mathrm{S} \rightarrow \mathrm{R}_{\mathrm{T}} \mathrm{R}_{\mathrm{T}} \mathrm{S}, \mathrm{UP} \mathrm{P}_{\mathrm{V}} \mathrm{S} \rightarrow \mathrm{R}_{\mathrm{T}} \mathrm{P}_{\mathrm{V}} \mathrm{S}$ \\
$\mathrm{C} \rightarrow \mathrm{R}_{\mathrm{T}}$ & $\mathrm{R}_{\mathrm{T}} \mathrm{CS} \rightarrow \mathrm{R}_{\mathrm{T}} \mathrm{R}_{\mathrm{T}} \mathrm{S}, \mathrm{R}_{\mathrm{A}} \mathrm{CS} \rightarrow \mathrm{R}_{\mathrm{A}} \mathrm{R}_{\mathrm{T}} \mathrm{S}, \mathrm{P}_{\mathrm{F}} \mathrm{CS} \rightarrow \mathrm{P}_{\mathrm{F}} \mathrm{R}_{\mathrm{T}} \mathrm{S}, \mathrm{CR} \mathrm{T} \rightarrow \mathrm{R}_{\mathrm{T}} \mathrm{R}_{\mathrm{T}} \mathrm{S}, \mathrm{CP}_{\mathrm{V}} \mathrm{S} \rightarrow \mathrm{R}_{\mathrm{T}} \mathrm{P}_{\mathrm{V}} \mathrm{S}$ \\
$\mathrm{C} \rightarrow \mathrm{P}_{\mathrm{F}}$ & $\mathrm{CR} \mathrm{R}_{\mathrm{T}} \mathrm{S} \rightarrow \mathrm{P}_{\mathrm{F}} \mathrm{R}_{\mathrm{T}} \mathrm{S}, \mathrm{CP} \mathrm{S} \rightarrow \mathrm{P}_{\mathrm{F}} \mathrm{P}_{\mathrm{V}} \mathrm{S}$ \\
$\mathrm{C} \rightarrow \mathrm{P}_{\mathrm{V}}$ & $\mathrm{R}_{\mathrm{T}} \mathrm{CS} \rightarrow \mathrm{R}_{\mathrm{T}} \mathrm{P}_{\mathrm{V}} \mathrm{S}, \mathrm{R}_{\mathrm{A}} \mathrm{CS} \rightarrow \mathrm{R}_{\mathrm{A}} \mathrm{P}_{\mathrm{V}} \mathrm{S}, \mathrm{P}_{\mathrm{F}} \mathrm{CS} \rightarrow \mathrm{P}_{\mathrm{F}} \mathrm{P}_{\mathrm{V}} \mathrm{S}$ \\
\hline
\end{tabular}

Any of the unique branches listed in Table B.4 are capable of providing the necessary constraint to the robot system for a lower mobility mode, with some configurations out performing others from kinematic and implementation point of view. From this we can further eliminate branch candidates. First, the $\mathrm{R}_{\mathrm{A}} \mathrm{P}_{\mathrm{V}} \mathrm{S}$ configuration is degenerate due to the alignment of the $\mathrm{R}_{\mathrm{A}}$ rotation and $\mathrm{P}_{\mathrm{V}}$ translation axes, which renders the branch unable to provide suitable planar motion, and is thus degenerate. Regarding the $\mathrm{R}_{\mathrm{A}} \mathrm{R}_{\mathrm{T}} \mathrm{S}$ branch, the resultant motion of the spherical joint is motion on the surface of a sphere. Although this motion is not degenerate, the motion of the spherical 
joint becomes increasingly difficult to implement with each additional degree-of-freedom removed from the platform. For example, a 3-DOF robot has three constrained branches in which the three associative spherical joints form a triangle. The vertices of this triangle must always lie on the sphere surfaces defined by the constrained branches, which could be impossible for many platform poses. Thus the $R_{A} R_{T} S$ constrained branch is too restrictive and is eliminated. Both constrained branches are derived from the $\mathrm{R}_{\mathrm{A}} \mathrm{CS}$ unconstrained branch which is therefore eliminated.

Elimination of the $\mathrm{R}_{\mathrm{A}} \mathrm{CS}$ unconstrained branch results in constrained branches in which the spherical joint experiences strictly planar motion. The ${ }_{T} R_{T} S, R_{T} P_{V} S, P_{F} R_{T} S$, and $P_{F} P_{V} S$ constrained branches each provide suitable motion, but are derived from different unconstrained branches. A lower mobility parallel robot can be formed by combining unconstrained branches in Table B.3, with suitable constrained branches from Table B.4, while satisfying the requirements imposed by Eq. (4.2) must be considered in addition to Eq. (4.1). 


\section{Appendix C}

\section{Prototype Motion Video}

In Chapter 6, still shots of the wing module were shown at various platform airfoil poses. Additionally, the screen shot of the Matlab Guide control interface was shown. The video from which the stills were taken can be retrieved at http://www.youtube.com/watch?v=QLkeZNP7UGs. The video shows the wing module prototype in use assuming a variety of poses. Additionally at the beginning of the video, a static stress test is performed. The video not only validates the developed kinematic formulation, but also highlights the benefits of using a truss system to perform wing morphing. The video was recorded on October 13, 2011 at Ryerson University. 


\section{References}

[1] C. F. Gray. Leonardo da vinci. http://en.wikipedia.org/wiki/File:Iranian_f-14.jpg, March 2012.

[2] Looper5920. Vmm-162 mv-22 osprey on the tarmac in iraq on april 12008. http://en.wikipedia.org/wiki/File:VMM-162_Osprey_on_the_tarmac_in_Iraq_on_ April_1-2008.JPG, September 2012.

[3] Adrian Pingstone. Concorde 216 (g-boaf). http://www.wingweb.co.uk/wingweb/img/ Concorde_G-BOAF_Filton.jpg, September 2012.

[4] Amirmgh. An iranian grumman f-14a tomcat exhibited in busheher, iran, in 2006. http: //en.wikipedia.org/wiki/File:Grumman_F-14_Tomcat.png, September 2012.

[5] A. K. Jha and J. N. Kudva. Morphing aircraft concepts, classifications, and challenges. In Proceedings of SPIE, volume 5388, pages 213-224, 2004.

[6] A. R. Rodriguez. Morphing aircraft technology survey. In 45th AIAA Aerospace Sciences Meeting and Exhibit, Reno, Nevada, 8-11 January 2007.

[7] S. P. Joshi, Z. Tidwell, W. A. Crossley, and S. Ramakrishnan. Comparison of morphing wing strategies based upon aircraft performance impacts. In 45th $A I A A / A S M E / A S C E / A H S / A S C$ Structures, Structural Dynamics \& Materials Conference, Palm Springs, California, 19-22 April 2004.

[8] Jr. J. D. Anderson. Aircraft performance and design. WCB/McGraw-Hill, 1999. 
[9] Jr. J. D. Anderson. Introduction to flight. McGraw-Hill Higher Education, 4th edition, 2000.

[10] Jr. J. D. Anderson. Fundamentals of aerodynamics. McGraw-Hill Higher Education, 3rd edition, 2011.

[11] D. A. Neal III, J. Farmer, and D. Inman. Aeroelastic modeling, analysis and testing of a morphing wing structure. In $4^{7} 7$ th $A I A A / A S M E / A S C E / A H S / A S C$ Structures, Structural Dynamics E Materials Conference, Newport, Rhode Island, 1-4 May 2006.

[12] S. Bharti, M. Frecker, G. Lesieutre, and J. Browne. Tendon actuated cellular mechanisms for morphing aircraft wing. In Proceedings of SPIE, volume 6523, 2007.

[13] G. R. Andersen, D. L. Cowan, and D. J. Piatak. Aeroelastic modeling, analysis and testing of a morphing wing structure. In 48th AIAA/ASME/ASCE/AHS/ASC Structures, Structural Dynamics \& Materials Conference, Honolulu, Hawaii, 23-26 April 2007.

[14] J. S. Flanagan, R. C. Strutzenberg, R. B. Myers, and J. E. Rodrian. Development and flight testing of a morphing aircraft, the nextgen mfx-1. In 48th AIAA/ASME/ASCE/AHS/ASC Structures, Structural Dynamics \& Materials Conference, Honolulu, Hawaii, 23-26 April 2007.

[15] D. T. Grant and R. Lind. Effects of time-varying inertias on flight dynamics of an asymmetric variable-sweep morphing aircraft. In AIAA Atmospheric Flight Mechanics Conference and Exhibit, Hilton Head, South Carolina, 20-23 August 2007.

[16] M. Abdulrahim and R. Lind. Control and simulation of a multi-role morphing micro air vehicle. In AIAA Guidance, Navigation, and Control Conference and Exhibit, San Francisco, California, 12-18 August 2005.

[17] M. Abdulrahim and R. Lind. Flight testing and response characteristics of a variable gullwing morphing aircraft. In AIAA Guidance, Navigation, and Control Conference and Exhibit, Providence, Rhode Island, 16-19 August 2004.

[18] M. Abdulrahim. Flight performance characteristics of a biologically-inspired morphing aircraft. In 43rd AIAA Aerospace Sciences Meeting and Exhibit, Reno, Nevada, 10-13 January 2005. 
[19] P. Bourdin, A. Gatto, and M. I. Friswell. The application of variable cant angle winglets for morphing aircraft control. In 24th Applied Aerodynamics Conference, San Francisco, California, 5-8 June 2006.

[20] N.Ameri, M. H. Lowenberg, and M. I. Friswell. Modelling the dynamic response of a morphing wing with active winglets. In AIAA Atmospheric Flight Mechanics Conference and Exhibit, Hilton Head, South Carolina, 20-23 August 2007.

[21] K. W. Morred, S. A. Taylor, T. K. Bliss, and H. Bart-Smith. Optimization of a tensegirty wing for biomimetic applications. In 45th IEEE Conference on Decision 6 Control, San Diego, California, 13-15 December 2006.

[22] Y. Ueno, K. Kawamura, and Y. Nakamura. Numerical simulation based on cfd and flight dynamics for morphing body. In 45th AIAA Aerospace Sciences Meeting and Exhibit, Reno, Nevada, 8-11 January 2007.

[23] L. Gonzalez. Morphing wing using shape memory alloy: a concept proposal. Technical report, University of Puerto Rico at Mayaguez, Mayaguez, Puerto Rico, Summer 2005.

[24] H. M. Garcia, M. Abdulrahim, and R. Lind. Roll control for a micro air vehicle using active wing morphing. In AIAA Guidance, Navigation, and Control Conference and Exhibit, Austin, Texas, 11-14 August 2003.

[25] M. Abdulrahim, H. Garcia, and R. Lind. Flight characteristics of shaping the membrane wing of a micro air vehicle. Journal of Aircraft, 42(1):131-137, 2005.

[26] M. Abdulrahim, H. Garcia, G. F. Ivey, and R. Lind. Flight testing a micro air vehicle using morphing for aeroservoelastic control. In 45th AIAA/ASME/ASCE/AHS/ASC Structures, Structural Dynamics \& Materials Conference, Palm Springs, California, 19-22 April 2004.

[27] R. Guiler and W. Huebsch. Wind tunnel analysis of a moprhing swept wing tailless aircraft. In 23rd AIAA Applied Aerodynamics Conference, Toronto, Ontario, 6-9 June 2005. 
[28] J. M. Lukens, G. W. Reich, and B. Sanders. Wing mechanization design and analysis for a perching micro air vehicle. In 49th AIAA/ASME/ASCE/AHS/ASC Structures, StructuresDynamics, ES Materials Conference, Schaumburg, Illinois, 7-10 April 2008.

[29] J. Blondeau, J. Richeson, and D. J. Pines. Design, development and testing of a morphing aspect ratio wing using an inflatable telescopic spar. In 44th $A I A A / A S M E / A S C E / A H S$ Structures, Structural Dynamics \&3 Materials Conference, Norfolk, Virginia, 7-10 April 2003.

[30] J. Blondeau Samuel and D. Pines. Design and testing of a pneumatic telescoping wing for unmanned aerial vehicles. Journal of Aircraft, 44(4):1088-1099, 2007.

[31] J.-S. Bae, T. M. Seigler, D. J. Inman, and I. Lee. Aerodynamic and aeroelastic considerations of a variable-span morphing wing. In 45th AIAA/ASME/ASCE/AHS/ASC Structures, Structural Dynamics 8 Materials Conference, Palm Springs, California, 19-22 April 2004.

[32] J.-S. Bae, T. M. Seigler, and D. J. Inman. Aerodynamic and static aeroelastic characteristics of a variable-span morphing wing. Journal of Aircraft, 42(2):528-534, 2005.

[33] J. Felício, P. Santos, P. Gamboa, and M. Silverstre. Evaluation of a variable-span morphing wing for a small uav. In 52nd AIAA/ASME/ASCE/AHS/ASC Structures, Structural Dynamics and Materials Conference, Denver, Colorado, 4-7 April 2011.

[34] J. Mestrinho, P. Gamboa, and P. Santos. Design optimization of a variable-span morphing wing for a small uav. In 52nd AIAA/ASMEASCE/AHS/ASC Structures, Structural Dynamics E Materials Conference, Denver, Colorado, 4-7 April 2011.

[35] J. Vale, F. Lau, A. Suleman, and P. Gamboa. Optimization of a morphing wing based on coupled aerodynamic and structural constraints. In 48th $A I A A / A S M E / A S C E / A H S / A S C$ Structures, Structural Dynamics \& Materials Conference, Honolulu, Hawaii, 23-26 April 2007.

[36] Y. Heryawan, H. C. Park, N. S. Goo, K. J. Yoon, and Y. H. Byun. Design and demonstration of a small expandable morphing wing. In Proceedings of SPIE, volume 5764, pages 224-231, 2005. 
[37] M Abdulrahim and R. Lind. Using avian morphology to enhance aircraft maneuverability. In AIAA Atmospheric Flight Mechanics Conference and Exhibit, Keystone, Colorado, 21-24 August 2006.

[38] H.-J. Su, D. Devaud, G. W. Reich, and J. Joo. Kinematic synthesis and design optimization of a mechanism concept for a perching mav. In 52nd AIAA/ASME/ASCE/AHS/ASC Structures, Structural Dynamics \& Materials Conference, Denver, Colorado, 4-7 April 2011.

[39] D. D. Smith, R. M. Ajaj, A. T. Isikveren, and M. I. Friswell. Multiobjective optimization for the multi-phase design of active polymorphing wings. In 52nd $A I A A / A S M E / A S C E / A H S / A S C$ Structures, Structural Dynamics \& Materials Conference, Denver, Colorado, 4-7 April 2011.

[40] D. S. Ramrakhyani, G. A. Lesieutre, M. Frecker, and S. Bharti. Aircraft structural morphing using tendon-actuated compliant cellular trusses. Journal of Aircraft, 42(6):1615-1620, 2005.

[41] D. Cadogan, T. Smith, F. Uhelsky, and M. MacKusick. Morphing inflatable wing development for compact package unmanned aerial vehicles. In 45th $A I A A / A S M E / A S C E / A H S / A S C$ Structures, Structural Dynamics \& Materials Conference, Palm Springs, California, 19-22 April 2004.

[42] D. Inoyama, B. P. Sanders, and J. J. Joo. Topology synthesis of distributed actuation systems for morphing wing structures. Journal of Aircraft, 44(4):1205-1213, 2007.

[43] D. Inoyama, B. P. Sanders, and J. J. Joo. Computational design of morphing wing structures through multiple-stage optimization process. In 48th $A I A A / A S M E / A S C E / A H S / A S C$ Structures, Structural Dynamics \& Materials Conference, Honolulu, Hawaii, 23-26 April 2007.

[44] D. R. Bye and P. D. McClure. Design of a morphing vehicle. In 48th AIAA/ASME/ASCE/AHS/ASC Structures, Structural Dynamics \& Materials Conference, Honolulu, Hawaii, 23-26 April 2007.

[45] M. H. Love, P. S. Zink, R. L. Stroud, D. R. Bye, S. Rizk, and D. White. Demonstration of morphing technology through ground and wind tunnel tests. In 48th 
AIAA/ASME/ASCE/AHS/ASC Structures, Structural Dynamics \& Materials Conference, Honolulu, Hawaii, 23-26 April 2007.

[46] T. G. Ivanco, R. C. Scott, M. H. Love, S. Zink, and T. A. Weisshaar. Validation of the lockheed martin morphing concept with wind tunnel testing. In 48th AIAA/ASME/ASCE/AHS/ASC Structures, Structural Dynamics \& Materials Conference, Honolulu, Hawaii, 23-26 April 2007.

[47] F. Xi, Y. Li, and H. Wang. Module-based method for design and analysis of reconfigurable parallel robots. Frontiers of Mechanical Engineering, 6(2):151-159, 2011.

[48] Z. M. Bi and L. Wang. Optimal design of reconfigurable parallel machining systems. Robotics and Computer-Integrated Manufacturing, 25:951-961, 2009.

[49] D. Gan, J. S. Dai, and Q. Liao. Constraint analysis on mobility change of a novel metamorphic parallel mechanism. Mechanism and Machine Theory, 45:1864-1876, 2010.

[50] D. Gan, J. S. Dai, and D. G. Caldwell. Constraint-based limb synthesis and mobility-changeaimed mechanism construction. ASME Journal of Mechanical Design, 133:051001-1-05101-9, 2011.

[51] J. Kotlarski, H. Abdellatif, T. Ortmaier, and B. Heimann. Enlarging the useable workspace of planar parallel robots using mechanisms of variable geometry. In ASME/IFToMM International Conference on Reconfigurable Mechanisms and Robots, pages 94-103, London, England, 22-24 June 2009.

[52] Z. M. Bi and B. Kang. Enhancement of adaptability of parallel kinematic machines with an adjustable platform. ASME Journal of Manufacturing Science and Engineering, 132:0610161-061016-9, 2010.

[53] S. G. Kumar, T. Nagarajan, and Y. G. Srinvasa. Characterization of reconfigurable stewart platform for contour generation. Robotics and Computer-Integrated Manufacturing, 25:721$731,2009$. 
[54] T. Sun, Y. Song, Y. Li, and J. Zhang. Workspace decomposition based dimensional synthesis of a novel hybrid reconfigurable robot. ASME Journal of Mechanisms and Robots, 2:031009$1-031009-8,2010$.

[55] A. K. Dash, I.-M. Chen, S. H. Yeo, and G. Yang. Workspace generation and planning singularity-free path for parallel manipulators. Mechanism and Machine Theory, 40(7):776805,2005 .

[56] F. Xi, Y. Xu, and G. Xiong. Design and analysis of a re-configurable parallel robot. Mechanism and Machine Theory, 41:191 - 211, 2006.

[57] A. D. Finistauri, F. Xi, and B. Petz. Architecture design and optimization of an on-thefly reconfigurable parallel robot. In H. Wu, editor, Parallel Manipulators: Towards New Applications, chapter 18, pages 279-404. InTech Education, 2008.

[58] A. D. Finistauri, F. Xi, and P.Walsh. Discretization method for the development of a modular morphing wing. AIAA Journal of Aircraft, 49(1):116-125, 2012.

[59] A. D. Finistauri and F. Xi. Reconfiguration analysis of a fully reconfigurable parallel robot. In J. S. Dai, M. Zoppi, and X. Kong, editors, Advances in Reconfigurable Mechanisms and Robots, volume I, pages 295-308. Springer, 2012.

[60] G. Liu, X. He, J. Yuan, and TBD. Development of a modular and reconfigurable robot with multiple working modes. In IEEE Int. Conf. on Robotics and Automation, 2008.

[61] A. Vertuan, G. Legnani, R. Admanini, D. Tosi, and N. Pedrocchi. Performance analysis of a reconfigurable redundant parallel manipulator. In $A S M E / I F T o M M$ International Conference on Reconfigurable Mechanisms and Robots, pages 593-601, London, England, 22-24 June 2009.

[62] A. D. Finistauri and F. Xi. Type synthesis and kinematics of a variable geometry truss mechanism for aircraft wing morphing. In ASME/IFToMM International Conference on Reconfigurable Mechanisms and Robots, pages 478-485, London, England, 22-24 June 2009. 
[63] H. Huang, Z. Deng, B. Li, and R. Lui. Analysis and synthesis of a kind of mobility reconfigurable robot with multi-task capability. Journal of Advanced Mechanical Design, Systems, and Manufacturing, 5(2):87-102, 2011.

[64] A. D. Finistauri and F. Xi. Reconfiguration analysis of a fully reconfigurable parallel robot. Accepted for publication in ASME Journal of Robotics, 2012.

[65] B. Stanford, M. Abdulrahim, R. Lind, and P. Ifju. Design and optimization of morphing mechanisms for highly flexible micro air vehicles. In $4^{\text {rth }}$ AIAA/ASME/ASCE/AHS/ASC Structures, Structural Dynamics, \& Materials Conference, Newport, Rhode Island, 1-4 May 2006.

[66] A. D. Finistauri, F. Xi, P. Walsh, and K. Behdinan. Wing line discretization for the development of a modular morphing wing. In 52nd AIAA/ASME/ASCE/AHS/ASC Structures, Structural Dynamics and Materials Conference, Denver, Colorado, 4-7 April 2011.

[67] R. L. Bishop. There is more than one way to frame a curve. The American Mathematical Monthly, 82(3):246-251, 1975.

[68] A. J. Hanson and H. Ma. Parallel transport approach to curve framing. Technical report, Department of Computer Science, Indiana University, Bloomington, Indiana, January 1995.

[69] L.-W. Tsai. Systematic enumeration of parallel manipulators. Technical report TR98-33, University of Maryland, Department of Mechanical Engineering, College Park, MD 20741, U.S.A, 1998.

[70] S. A. Joshi and L.-W. Tsai. Jacobian analysis of limited-dof parallel manipulators. ASME Journal of Mechanical Design, 124(2):254-258, 2002.

[71] F. Xi, D. Zhang, Z. Xu, and C. M. Mechefske. A comparative study on tripod units for machine tools. International Journal of Machine Tools and Manufacture, 43:721-730, 2003.

[72] A. Fattah and A. M. Hasan Ghasemi. Isotropic design of spatial parallel manipulators. International Journal of Robotic Research, 21(9):811-824, 2002. 
[73] J. Angeles and C. S. López-Cajún. Kinematic isotropy and conditioning index of serial robotic manipulators. International Journal of Robotic Research, 11(6):560-571, 1992.

[74] J. P. Merlet. Jacobian, manipulability, condition number, and accuracy of parallel robots. ASME Journal of Mechanical Design, 128(1):199-206, 2006.

[75] Inc. Firgelli Technologies. L16 series datasheet. http://www.firgelli.com/Uploads/L16_ datasheet.pdf, August 2012.

[76] M. T. Kikuta. Mechanical properties of candidate materials for morphing wings. Master's thesis, Virginia Polytechnic Institute and State University, Virginia, USA, 2003.

[77] D. Zhang and L. Wang. Conceptual development of an enhanced tripod mechanism for machine tool. Robotics and Computer-Integrated manufacture, 21:318-327, 2005. 